\title{
Passive Time-Domain Macromodels of High Speed Interconnect Networks
}

\author{
by
}

Anestis Dounavis

\begin{abstract}
A thesis submitted to the
Faculty of Graduate Studies and Research

in partial fulfillment of the requirements

for the degree of

Doctor of Philosophy
\end{abstract}

Ottawa-Carleton Institute for Electrical Engineering,

Department of Electronics,

Carleton University,

Ottawa, Ontario, Canada

December, 2003

C Anestis Dounavis 2003 


\author{
National Library \\ of Canada \\ Acquisitions and \\ Bibliographic Services \\ 395 Wellington Street \\ Ottawa ON K1A ON4 \\ Canada
}

Bibliothèque nationale

du Canada

Acquisisitons et services bibliographiques

395 , rue Wellington Ottawa ON K1A ON4 Canada
Your file Votre référence ISBN: 0-612-89887-3

Ourfile Notre référence ISBN: 0-612-89887-3
The author has granted a nonexclusive licence allowing the National Library of Canada to reproduce, loan, distribute or sell copies of this thesis in microform, paper or electronic formats.

The author retains ownership of the copyright in this thesis. Neither the thesis nor substantial extracts from it may be printed or otherwise reproduced without the author's permission.
L'auteur a accordé une licence non exclusive permettant à la Bibliothèque nationale du Canada de reproduire, prêter, distribuer ou vendre des copies de cette thèse sous la forme de microfiche/film, de reproduction sur papier ou sur format électronique.

L'auteur conserve la propriété du droit d'auteur qui protège cette thèse. $\mathrm{Ni}$ la thèse ni des extraits substantiels de celle-ci ne doivent être imprimés ou aturement reproduits sans son autorisation.
In compliance with the Canadian Privacy Act some supporting forms may have been removed from this dissertation.

While these forms may be included in the document page count, their removal does not represent any loss of content from the dissertation.
Conformément à la loi canadienne sur la protection de la vie privée, quelques formulaires secondaires ont été enlevés de ce manuscrit.

Bien que ces formulaires aient inclus dans la pagination, il n'y aura aucun contenu manquant. 


\begin{abstract}
As operating frequencies increase and device sizes decrease, the complexity of modern circuits are putting enormous pressures on computer aided design tools. In the VLSI area, interconnect effects such as signal delay, cross-talk, ringing, reflections and distortion can severely effect the signal integrity of designs. In addition, susceptibility of high-speed circuits to incident fields can also influence the signal integrity of networks. Consequently, circuit designers must consider interconnect analysis to ensure circuit performance and reliability. However, the modeling of high-speed interconnects using conventional circuit simulators such as SPICE can become prohibitively CPU expensive.

In this thesis, efficient algorithms for the simulation of high-speed interconnects are presented. The methods are based on a matrix rational approximation (MRA) of an exponential function used to describe the solution of Telegrapher's equation. The transmission line parameters of the macromodel can be lossy, coupled and frequency-dependent. An advantage of the MRA algorithm is that the macromodel can be formulated analytically in terms of predetermined coefficients and the per-unit-length parameters. In addition the proposed algorithm is passive. Passivity is an important property to satisfy to ensure stability of the overall network. The MRA algorithm is also compatible with passive model order reduction techniques based on congruence transformations. In addition, the MRA algorithm can be used to perform sensitivity analysis and to predict the effect of incident fields on interconnects.
\end{abstract}




\section{Acknowledgment}

I wish to extent my appreciation and gratitude to Professor Michel Nakhla for his knowledge, experience and kindness. His invaluable advice during supervision made the work of this thesis less difficult and more enjoyable.

I would like to thank Professor Ram Achar of Carleton University and Ibrahim M. Elfadel of IBM T. J. Watson Research Center for many useful discussions on macromodeling algorithms. Their ideas, comments and suggestions were always appreciated. I wish to thank the following colleagues for making Carleton an enjoyable experience: Pramil Agorwal, Mark Cannon, Emad Gad, Richard Griffith, Pavan Gunupudi, Kamal Harb, Ihsan Irdin, Roni Khazaka, Xin Li, Christina Oregon, Dharmendra Saraswat, Bojan Skiljo and Nick Soveiko. In addition, I would like to thank the professors, staff and students at the department of electronics for their supportive and friendly manner.

My final thoughts are with my parents, Thomas and Catina, my brother Vasilios and sister Anthoula. Without their encouragement and endless support, I would not have had the opportunity to complete this study. 


\section{Contents}

1. Introduction 1

1.1 Background and Motivation. . . . . . . . . . . . . . .

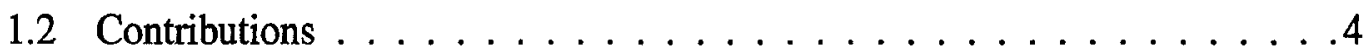

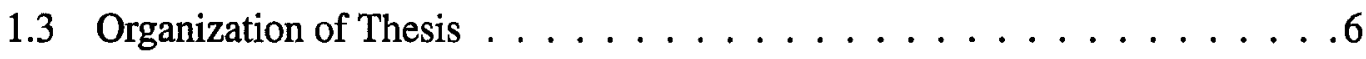

2. Interconnect Modeling and Simulation 7

2.1 Introduction . . . . . . . . . . . . . . . .

2.2 Interconnect Models. . . . . . . . . . . . . . . .

2.2.1 Quasi-Transverse Electromagnetic Models . . . . . . . . . . . .9

2.2.2 Full Wave Models. . . . . . . . . . . . . . . . . . 10

2.3 Frequency-Domain Analysis . . . . . . . . . . . . 11

2.3.1 Eigen-Decomposition Based Transmission Line Solution . . . . . 12

2.3.2 Matrix-Exponential Based Transmission Line Solution . . . . . . 15

2.4 Formulation of Circuit Equations . . . . . . . . . . . . . 16

2.4 .1 Linear Networks. . . . . . . . . . . . . . . . . . 16

2.4 .2 Nonlinear Networks. . . . . . . . . . . . . . . 18

2.4.3 Distributed Nonlinear Networks . . . . . . . . . . . . 19

2.5 Simulation Techniques Based on Transmission Line Macromodels . . . . 21

2.5.1 Conventional Lumped Segmentation . . . . . . . . . . . . 22 
2.5.2 Method of Characteristics. . . . . . . . . . . . 23

2.5 .3 Least Square Optimization . . . . . . . . . . . . 25

2.5.4 Basis Function Macromodels . . . . . . . . . . . . . 26

2.5.5 Compact Finite-Differences Based Approximation. . . . . . . . . 27

2.5.6 Integrated Congruence Transforms . . . . . . . . . . . . 28

2.6 Simulation Techniques Based on Model Order Reduction . . . . . . . . 29

2.6.1 Explicit Moment Matching . . . . . . . . . . . . . . 30

2.6.2 Implicit Moment Matching . . . . . . . . . . . 32

3. General Class of Passive Macromodels for High Speed Interconnects 34

3.1 Introduction . . . . . . . . . . . . . . . . . 34

3.2 Development of the MRA Macromodel . . . . . . . . . . . 35

3.3 Passivity Considerations . . . . . . . . . . . . . . . 36

3.3.1 Review of Passivity Properties . . . . . . . . . . . 36

3.3 .2 Preserving Passivity . . . . . . . . . . . . . . . 37

3.4 Classes of Rational Approximations . . . . . . . . . . . . 43

3.4.1 Padé Rational Approximation. . . . . . . . . . . . . . 43

3.4.2 Minimax Rational Approximation . . . . . . . . . . . 44

3.5 Criteria for Selecting the Order of Approximation . . . . . . . . . . 45

4. Development of Time-Domain Macromodel 47

4.1 Introduction . . . . . . . . . . . . . . . 47

4.2 Development of Time-Domain Macromodel . . . . . . . . . . . . 48

4.2.1 Formulation of Circuit Equations . . . . . . . . . . . 48

4.2.2 Formulation of MRA Macromodel . . . . . . . . . . . . . 49

4.3 Circuit Realization of MRA Macromodel . . . . . . . . . . . 52

4.3 .1 RC Transmission Lines . . . . . . . . . . . . . . . 52

4.3.2 Lossless Transmission Lines . . . . . . . . . . . . . . . 55 
4.3.3 Lossy Transmission Lines. . . . . . . . . . . . . . . . . . 58

4.3.4 Example of MRA Circuit Formulation . . . . . . . . . . . . 62

4.4 Application of MRA to Incident Field Coupling . . . . . . . . . . . . . 64

4.4.1 Formulation of Incident Field Forcing Function . . . . . . . . . . 64

4.4.2 Time-domain Macromodel for Equivalent Sources. . . . . . . . . 67

4.5 Numerical Examples . . . . . . . . . . . . . . . . . . 70

4.5 .1 Example 4.1. . . . . . . . . . . . . . 70

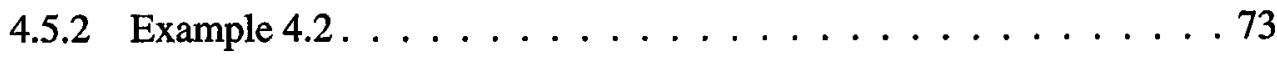

4.5 .3 Example $4.3 \ldots \ldots \ldots \ldots \ldots$

4.5.4 Example 4.4. . . . . . . . . . . . . 77

5. Frequency-Dependent Parameters $\quad 81$

5.1 Introduction . . . . . . . . . . . . . . 81

5.2 Behaviour of Per-Unit-Length Parameters . . . . . . . . . . . . 82

5.3 Modeling of Frequency-Dependent Parameters . . . . . . . . . . 85

5.4 Circuit Realization for Frequency-Dependent Parameters . . . . . . . . . 91

5.5 Numerical Examples . . . . . . . . . . . . . . . . . . . 97

5.5 .1 Example $5.1 \ldots \ldots \ldots \ldots$. . . . . . . . . . . . . .

5.5 .2 Example $5.2 \ldots \ldots \ldots \ldots 10 \ldots \ldots$

6. Addressing Transient Errors in Passive Rational Macromodels 104

6.1 Introduction . . . . . . . . . . . . . . . . . 104

6.2 Concepts Pertinent to the Proposed Algorithm . . . . . . . . . . . . 105

6.2 .1 Concept . . . . . . . . . . . . . . 106

6.2.2 Limitations of Current Passive Macromodeling Algorithms . . . . 107

6.3 Development of the Proposed Macromodeling Algorithm . . . . . . . . 109

6.3.1 Two-Conductor Transmission Line Case . . . . . . . . . . . . 111

6.3.2 Controlling Asymptotic Behaviour of Impulse Response . . . . . 111 
6.3.3 Extension to Multi-conductor Transmission Lines . . . . . . . . . 112

6.3.4 Computation of Predetermined Coefficients . . . . . . . . . 114

6.3.5 Preserving Passivity. . . . . . . . . . . . . . 118

6.4 Development of Time-Domain Transmission Line Macromodel . . . . . . 119

6.4.1 Formulation of Time-Domain Macromodel . . . . . . . . . . . 119

6.4.2 Circuit Realization of MRA Macromodel . . . . . . . . . . 120

6.5 Examples . . . . . . . . . . . . . . . . . 126

6.5 .1 Example 6.1........................ 126

6.5 .2 Example $6.2 \ldots \ldots \ldots \ldots . \ldots \ldots$

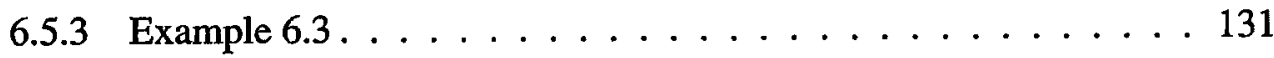

7. Passive Model Reduction of Distributed Transmission Line Networks $\quad 133$

7.1 Introduction . . . . . . . . . . . . . . 133

7.2 Formulation of Network Equations . . . . . . . . . . . . . 134

7.3 Model Reduction and Passivity Preservation . . . . . . . . . . . . 136

7.3.1 Model Reduction . . . . . . . . . . . . . . . . 137

7.3.2 Passivity Preservation . . . . . . . . . . . . . . 137

7.3.3 Transmission Lines with Frequency-Dependent Parameters . . . . 146

7.4 Examples . . . . . . . . . . . . . . . . . . 146

7.4 .1 Example $7.1 \ldots \ldots \ldots . \ldots \ldots 147 \ldots \ldots \ldots$

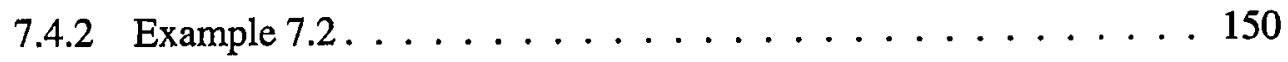

8. Sensitivity Analysis of Transmission Lines with Nonlinear Terminations 153

8.1 Introduction . . . . . . . . . . . . . . . . . 153

8.2 Formulation of Network Equations . . . . . . . . . . . . . . 154

8.3 Sensitivity Analysis . . . . . . . . . . . . . . . 155

8.3.1 Sensitivity Analysis of Linear Circuits . . . . . . . . . . 155

8.3.2 Sensitivity Analysis with Nonlinear Terminations . . . . . . . 156 
8.3.3 Sensitivity with Respect to Physical Transmission Line Parameters 157

8.3.4 Calculating the derivatives of the MNA matrices . . . . . . . 157

8.4 Examples . . . . . . . . . . . . . . . . . . . 159

8.4 .1 Example 8.1. . . . . . . . . . . . . . 159

8.4 .2 Example $8.2 \ldots \ldots \ldots 3$

$\begin{array}{ll}\text { 9. Summary and Future Work } & 167\end{array}$

9.1 Summary . . . . . . . . . . . . . . . . . . . 167

9.2 Future Work. . . . . . . . . . . . . . . . . . . . 169

$\begin{array}{ll}\text { Reference } & 171\end{array}$ 


\section{List of Figures}

2.1 Interconnect hierarchy $\ldots \ldots \ldots \ldots \ldots$

2.2 Top view and cross-sectional view of an interconnect system . . . . . . . . .9

2.3 Multi-conductor transmission line . . . . . . . . . . . . . 11

2.4 Linear circuit example . . . . . . . . . . . . . . . . . 17

2.5 Rectifier circuit with nonlinear capacitor . . . . . . . . . . 18

2.6 Distributed nonlinear circuit example. . . . . . . . . . . . . . 19

2.7 Lumped transmission line model . . . . . . . . . . . . . . . 22

2.8 Method of characteristics transmission line model.. . . . . . . . . . . . . 25

3.1 Pole-zero distribution for scalar rational approximation of $(3.7) \ldots . \ldots 39$

4.1 MRA macromodel represented in terms of the subsections of $(4.9) \ldots \ldots 1$

4.2 Realization of two-conductor $\mathrm{RC}$ transmission line . . . . . . . . . . 53

4.3 Realization of RC MTL . . . . . . . . . . . . . . . . . . . 54

4.4 Realization of two-conductor lossless transmission line . . . . . . . . . 56

4.5 Realization of lossless MTL . . . . . . . . . . . . . . . 57

4.6 Realization of two-conductor lossy transmission line . . . . . . . . . . . 60

4.7 Realization of lossy MTL for complex pole-zero subsection of (4.24) . . . . . . 61

4.8 Realization of lossy MTL for real pole-zero subsection of $(4.25)$. . . . . . . . 62

4.9 Example circuit formulation of MRA macromodel. . . . . . . . . . . . 63

4.10 Definition of parameters for incident field coupling . . . . . . . . . 65

4.11 Equivalent circuit of incident field coupling to transmission-lines . . . . . . 68 
4.12 Coupled RC distributed network (Example 4.1) . . . . . . . . . . . 71

4.13 Transient response (Example 4.1) . . . . . . . . . . . . . 72

4.14 Coupled transmission line network (Example 4.2) . . . . . . . . . . 73

4.15 Frequency response (Example 4.2) . . . . . . . . . . . . . . . 74

4.16 Transient response (Example 4.2) . . . . . . . . . . . . . . . . . 74

4.17 Seven transmission line network (Example 4.3) . . . . . . . . . . . 75

4.18 Transient response of nonlinear network (Example 4.3) . . . . . . . . . . 76

4.19 Coupled Lossy Microstrip Line . . . . . . . . . . . . . . . . . 78

4.20 Linear test circuit . . . . . . . . . . . . . . . . . . 78

4.21 Coupled Voltage Waveform for The Linear Circuit. . . . . . . . . . . . 79

4.22 Coupled transmission-line with non-linear terminations (Example 8.1) . . . . 79

4.23 Output Waveform of The Nonlinear Test Circuit . . . . . . . . . . . . 80

5.1 Cross-sectional geometry and dimensions of stripline interconnect . . . . . 83

5.2 P.u.l. resistance and inductance versus frequency. . . . . . . . . . . . . . . 84

5.3 P.u.l. conductance and capacitance versus frequency . . . . . . . . . . . . . 84

5.4 Modeling of $\mathrm{R}(\mathrm{s})+\mathrm{sL}(\mathrm{s}) \ldots \ldots \ldots \ldots$. . . . . . . . . . . . . . . . . .

5.5 Modeling of $\mathrm{G}(\mathrm{s})+\mathrm{sC}(\mathrm{s}) \ldots \ldots \ldots \ldots \ldots$. . . . . . . . . . . . . . . .

5.6 Modeling of $\mathrm{R}(\mathrm{s})+\mathrm{sL}(\mathrm{s})$ for MTL . . . . . . . . . . . . . 88

5.7 Modeling of $\mathrm{G}(\mathrm{s})+\mathrm{sC}(\mathrm{s})$ for MTL . . . . . . . . . . . . . . . . . 90

5.8 Realization of two-conductor transmission-line with frequency-dependent parameters for complex pole-zero subsection of $(5.5) \ldots \ldots 9$

5.9 Realization of two-conductor transmission-line with frequency-dependent parameters for the real pole-zero subsection of $(5.6) \ldots \ldots 9$

5.10 Realization of MTL with frequency-dependent parameters for complex pole-zero subsection of $(5.5) \ldots \ldots 95$

5.11 Realization of MTL with frequency-dependent parameters for real pole-zero

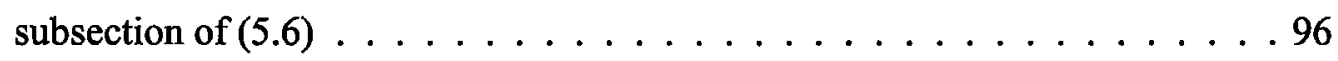

5.12 Nonlinear circuit with frequency-dependent parameters (Example 5.1) . . . . 98

5.13 Fitting of the p.u.l parameters (Example 5.1) . . . . . . . . . . . 99 
5.14 Transient response of nonlinear circuit (Example 5.1) . . . . . . . . . 100

5.15 Fitting of the p.u.l. parameters (Example 5.2) . . . . . . . . . . . . . . 102

5.16 Transient response of nonlinear network (Example 5.2) . . . . . . . . . 103

6.1 MRA macromodel representation. . . . . . . . . . . . . . . . 121

6.2 Realization of two-conductor transmission-line for $(6.42) \ldots \ldots$. . . . . . . 122

6.3 Realization of two-conductor transmission-line for $(6.44) \ldots \ldots$. . . . . . 123

6.4 Realization of MTL for $(6.42) \ldots \ldots \ldots$. . . . . . . . . . . 124

6.5 Realization of MTL for $(6.44) \ldots \ldots \ldots \ldots$

6.6 Frequency response $($ Example 6.1) . . . . . . . . . . . . . . . 127

6.7 Transient response IFFT versus PRIMA (Example 6.1) . . . . . . . . . . . 127

6.8 Transient response IFFT versus proposed macromodel (Example 6.1). . . . . 128

6.9 Lossy coupled transmission-line network (Example 6.2) . . . . . . . . . . . 129

6.10 Transient response, method of characteristics vs MRA (Pade coefficients) (Example 6.2) . . . . . . . . . . . . . . . . . . 130

6.11 Transient response, method of characteristics vs proposed (Example 6.2) . . 130

6.12 Long low loss line with frequency dependent parameters (Example 6.3) . . . 131

6.13 Time Domain Response (Example 6.3) . . . . . . . . . . . . . . . . 132

7.1 Linear subnetwork circuit with nonlinear termination (Example 7.1) . . . . 147

7.2 Y-parameters of linear subnetwork (Example 7.1) . . . . . . . . . . . 148

7.3 Transient Response (Example 7.1) . . . . . . . . . . . . . . . . . . . . 149

$7.4 \mathrm{R}$ and $\mathrm{L}$ versus frequency (Example 7.2$) \ldots \ldots . \ldots . \ldots$

7.5 Y-parameters of linear subnetwork (Example 7.2) . . . . . . . . . . . 151

7.6 Transient response (Example 7.2) . . . . . . . . . . . . . . . . 152

8.1 Transmission line system with nonlinear termination (Example 8.1) $\ldots \ldots$

8.2 Transient response of nonlinear circuit (Example 8.1) . . . . . . . . . 161

8.3 Sensitivity with respect to $\mathrm{C} 11 \ldots \ldots . \ldots \ldots 2$

8.4 Three coupled transmission lines with nonlinear terminations . . . . . . . 165

8.5 Physical/geometrical parameters of microstrip line. . . . . . . . . . . 165

8.6 Signal response before and after optimization (example 8.2) . . . . . . . 166 


\section{List of Tables}

4.1 Accuracy vs. Efficiency (Example 4.1) . . . . . . . . . . . . 71

5.1 P.u.l. parameters (Example 5.1) . . . . . . . . . . . . . . . . . 98

5.2 P.u.1. parameters (Example 5.2) . . . . . . . . . . . . . . . 101

6.1 P.u.l. parameters of Example $6.2 \ldots \ldots$. . . . . . . . . . . . 129

6.2 P.u.l. parameters of Example 6.3 . . . . . . . . . . . . . . 131

7.1 CPU comparison of transient response (Example 7.1) . . . . . . . . . . 147

7.2 CPU comparison of transient response (Example 7.2) . . . . . . . . . . 150

8.1 Example showing calculations of sensitivity network . . . . . . . . 158

8.2 Computational Complexity: Proposed vs Perturbation (Example 8.1) . . . . 160 


\section{Abbreviations}

AWE Asymptotic waveform evaluation.

$\mathrm{CFH}$ Complex frequency hopping.

IFFT Inverse fast fourrier transform.

KCL Kirchoff's current law.

LU Lower-upper matrix decomposition.

MNA Modified nodal analysis.

MOR Model-order-reduction.

MRA Matrix rational approximation.

MTL Multi-conductor transmission line.

ODE Ordinary differential equation.

PDE Partial differential equation.

PEEC Partial element equivalent circuit.

PRIMA Passive reduced-order interconnect macromodeling algorithm.

p.u.l. Per-unit-length.

RC Resistor, capacitor.

RL Resistor, inductor.

rPEEC Retarded partial element equivalent circuit.

TEM Transverse electromagnetic. 


\section{Chapter 1}

\section{Introduction}

\subsection{Background and Motivation}

The phenomenal growth in density, operating speeds and complexity of modern integrated circuits has made interconnect analysis a requirement for all state-of-the-art circuit simulators. As frequency increases, interconnects behave like transmission lines and effects such as ringing, signal delay, distortion, attenuation and crosstalk can severely degrade signal integrity [1]-[4]. Interconnects can exist at various levels of design hierarchy such as on-chip, packaging structures, multi-chip modules, printed-circuit-boards and backplanes. For many high-speed electrical networks, overall performance may depend mostly on the delay of interconnects rather than the delay of devices. In addition, susceptibility of high-speed circuits to incident fields can also influence the signal integrity of networks [2]. Consequently, designers must consider interconnect analysis at the early stages of the design cycle to ensure circuit performance and reliability.

There are two major difficulties in modeling distributed transmission lines in a circuit simulation environment. The first difficulty is due to the mixed frequency/time problem, which is encountered while linking distributed transmission lines with nonlinear circuit elements. This is because, distributed transmission lines are described by partial differential equations (PDEs) that are best solved in the frequency-domain, whereas nonlinear ele- 
ments are described only in the time-domain as nonlinear ordinary differential equations (ODEs). Hence, it is essential to model interconnects such that they can be directly included in a nonlinear circuit simulation environment. The second difficulty is due to the excessive CPU time associated with the simulation of transmission lines.

A significant amount of research has been done to address the simulation of distributed interconnects in the presence of nonlinear elements [1]-[45]. Approaches based on conventional lumped segmentation of transmission lines provide a brute force solution to the problem of mixed frequency/time simulation [2]. However, these methods lead to large circuit matrices, rendering the simulation inefficient. There are two simulation strategies for modeling transmission lines. One approach is based on model-order-reduction (MOR) techniques [6]-[17] and the other is based on developing efficient macromodels for transmission lines [18]-[33].

MOR techniques are based on moment matching and Padé approximations. The goal of these methods is to approximate the external behavior of large distributed linear networks with fewer equations, while maintaining the accuracy of the response. This is achieved by extracting only the dominant poles and residues of the network. MOR techniques such as asymptotic waveform evaluation (AWE) [6]-[8], complex frequency hopping (CFH) [9], Padé via Lanczos [10], Arnoldi [11]-[12], and passive reduced-order interconnect macromodeling algorithm (PRIMA) [13] have proven to be efficient means to reduce large linear interconnect networks. However, the efficiency of these methods drops significantly as the number of ports increases. Recently, time-domain circuit reduction techniques that reduce entire distributed networks including nonlinear/linear terminations have been proposed as solution algorithms of multi-port distributed networks [17].

In addition to MOR techniques, macromodeling of interconnects has been a topic of intense research [18]-[33]. The development of an efficient general-purpose transmission line macromodel allows circuit designers to efficiently evaluate their design within the familiar framework of a circuit simulation environment. This enable the circuit simulator to treat interconnects as regular circuit components regardless of what the electrical parame- 
ters might be. The complexity of modeling interconnects depends on the operating frequency and physical structure. At high frequencies, the length of interconnects become a significant fraction of the operating wavelength, and distributed models are required. Skin and proximity effects also become prominent at high frequencies requiring the per-unitlength (p.u.1.) parameters to be functions of frequency [2]-[4], [34]-[35].

One of the key issues of modeling transmission lines is the preservation of passivity. Since transmission lines are passive elements, passivity is an important property to satisfy because stable but not passive macromodels can produce unstable networks when connected to other passive loads. This can lead to spurious oscillations during transient analysis. In addition, accurate passive models are highly desirable by passive MOR techniques that require passive discretization of transmission lines to preserve passivity [12]-[13], [32]-[33].

Another important issue is the modeling of high-speed circuits exposed to incident fields [2], [36]-[42]. Electromagnetic interference can induce currents in circuits that may cause improper switching of devices. As packing densities increase, incident field coupling between adjacent modules can also affect the signal integrity of the overall design. Hence, it becomes important to model transmission lines exposed to incident fields.

For large distributed networks optimization may become necessary to improve performance and reliability. Macromodels that perform sensitivity analysis with respect to interconnect parameters can become powerful tools for optimizers [43]-[45]. For example, interconnects play an important role in determining the circuit's density, power consumption and clock frequency. Increasing the circuit's density leads to shorter interconnects which reduces the problem of delay and reflections; however, this leads to greater crosstalk between adjacent lines. Designers must make proper trade-offs, often between conflicting design requirements to obtain the best possible performance. Hence, efficient and accurate sensitivity information with respect to interconnect parameters is required by optimizers to solve nonlinear circuits with embedded distributed transmission lines. 


\subsection{Contributions}

The subject of this thesis is the development of a general-purpose passive transmission line macromodel [46]-[69]. The macromodel can be formulated analytically in terms of predetermined coefficients and the p.u.l. parameters of transmission lines. The p.u.l. parameters of the macromodel can be lossy, coupled and frequency-dependent. The proposed algorithm is suitable for passive MOR techniques based on congruent transformations [53]-[58]. Sensitivity analysis of transmission lines can also be derived using the proposed algorithm [59]-[61]. In addition, the macromodel is extended to analyze incident field coupling to transmission lines [64]-[65]. The contributions of this thesis are:

1. A general class of passive macromodeling algorithm for multi-port distributed interconnects is presented. The algorithm is based on a matrix rational approximation (MRA) of an exponential function describing Telegrapher's equations. A new theorem is described that specifies sufficient conditions for the MRA macromodel to generate passive macromodels [46], [49]. Classes of rational approximations are described that satisfy the conditions of the passivity theorem. The first is based on a Padé rational approximation and the other is based of minimax approximation. A key feature of the proposed algorithm is that the macromodel is formulated analytically in terms of predetermined coefficients and the p.u.l. parameters of transmission lines. The time-domain formulations are provided for the MRA macromodel [46], [52]. The realizations are described both in terms of circuit elements and in terms of the modified nodal analysis (MNA) equations.

2. A technique to model interconnects with frequency-dependent parameters is described for the MRA algorithm [52]. The method uses positive real rational functions to fit the frequency dependent parameters. It can be proven mathematically that these functions preserve passivity of the macromodel. The time-domain macromodel for transmission lines with frequency-dependent parameters is also provided.

3. A time-domain macromodel for incident field coupling of lossy multi-conductor transmission lines (MTLs) is presented [64]-[65]. The MRA algorithm is used to 
model both the transmission line and incident fields. The macromodel takes the form of ODEs and can easily be included in a circuit simulation environment.

4. Most rational macromodels employ some kind of approximation in the frequencydomain to match the impulse response up to some maximum frequency range of interest. However, the behaviour after the maximum frequency is generally not considered, which can lead to significant errors in the impulse transient response (especially in the early-time period). This can affect the accuracy of the transient response at all other time points when the macromodel is included during simulation in large networks. In order to address the above problem, a new algorithm is described which provides a mechanism to control the asymptotic behaviour of the high frequency impulse response while matching the response to the maximum frequency range of interest [62]-[63]. The new transmission line macromodel leads to significant reduction in errors in transient simulation. The method is also based on a matrix rational approximation of an exponential function. The algorithm is passive and can also be formulated analytically in terms of predetermined coefficients and the p.u.l. parameters.

5. A new technique is described to advance Krylov-subspace based passive MOR techniques to include distributed interconnects [54]-[56]. The method uses the MRA algorithm to model transmission lines. A proof is provided showing that the contribution of the MRA stamp to the MNA equations lead to a passive reduced-order macromodel.

6. Sensitivity analysis of MTLs in the presence of nonlinear terminations is described [59]-[61]. Sensitivity information is extracted using the MRA algorithm. The method enables analyses of interconnect structures with both electrical and physical parameters. An important feature of the proposed approach is that the derivatives of the MNA matrices with respect to the p.u.l. parameters are obtained analytically. 


\subsection{Organization of Thesis}

The organization of this thesis is as follows. A brief review of high-speed interconnects, including frequency and time-domain formulations are provided in Chapter 2. In Chapter 3, a new general class of passive macromodeling algorithm for multi-port distributed interconnects is described. The algorithm is based on the MRA, used to describe the solution of Telegrapher's equations. The time-domain formulation is presented in Chapter 4. In addition, the modeling of incident field coupling to lossy MTLs is described. Chapter 5 extends the MRA to include transmission lines with frequency-dependent p.u.l. parameters. A new algorithm to minimize high frequency errors in transient responses of rational macromodels is presented in Chapter 6. Chapter 7 provides a technique to advance Krylov-subspace based passive MOR methods to include distributed interconnects. Sensitivity analysis of MTLs in the presence of nonlinear terminations is described in Chapter 8. Chapter 9 presents a summary and a list of future work. 


\section{Chapter 2}

\section{Interconnect Modeling and}

\section{Simulation}

\subsection{Introduction}

Interconnects are electrical conducting structures that propagate signals. At low frequencies, interconnects behave as short-circuits. However, as frequency increases, interconnects gradually display resistive, capacitive and inductive effects. These effects can severely degrade the signal integrity of networks. Depending on the operating frequency and physical structure of interconnects different transmission line models are required. Each model offers different challenges to circuit simulators. The goal of this chapter is to review some of the interconnect models and solution algorithms that are used for interconnect analysis.

This chapter is organized as follows. The various transmission line models used for interconnect analysis are presented is section 2.2. Section 2.3 describes the frequency-domain solution of multi-conductor transmission lines (MTLs). The formulations of circuit and transmission line equations are presented in section 2.4. Section 2.5 provides several transmission line macromodels for nonlinear circuit analysis. Model-order-reduction (MOR) algorithms are briefly described in section 2.6 . 


\subsection{Interconnect Models}

Interconnects are present at different levels of technology such as digital chips, electrical packages, multi-chip modules, printed-circuit-boards and backplanes (Figure 2.1). Examples of interconnects are microstrip lines, striplines and coaxial cables [2], [4]. The physical structure of a microstrip line network consisting of three conductors and ground plane is shown in Figure 2.2.

To simulate interconnects with circuit elements, electrical models are required. The selection of the model depends on the physical interconnect structure as well as the operating frequency of the circuit. These two factors determine whether the modeling of interconnects is based on quasi-transverse electromagnetic (quasi-TEM) or full wave assumptions.

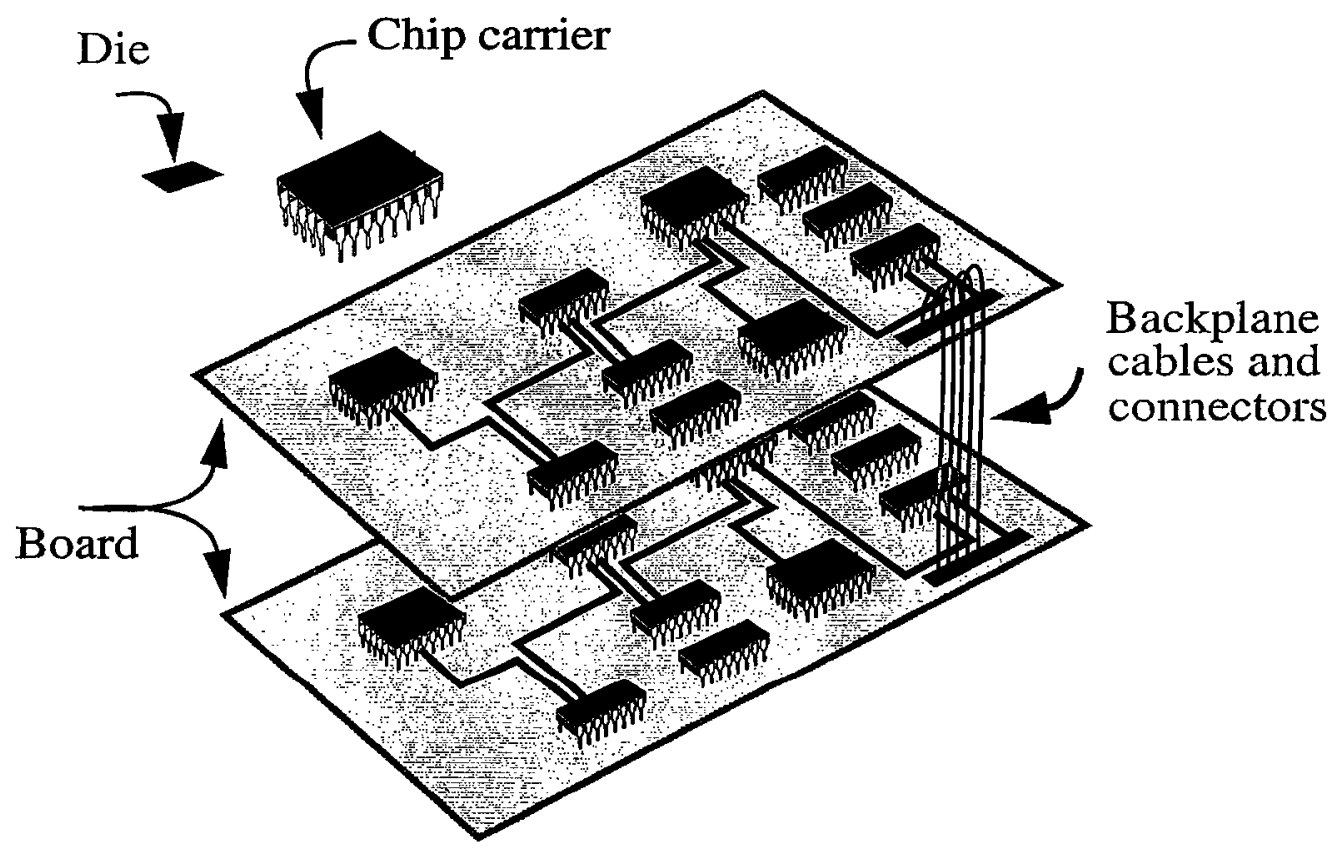

Figure 2.1 Interconnect hierarchy 

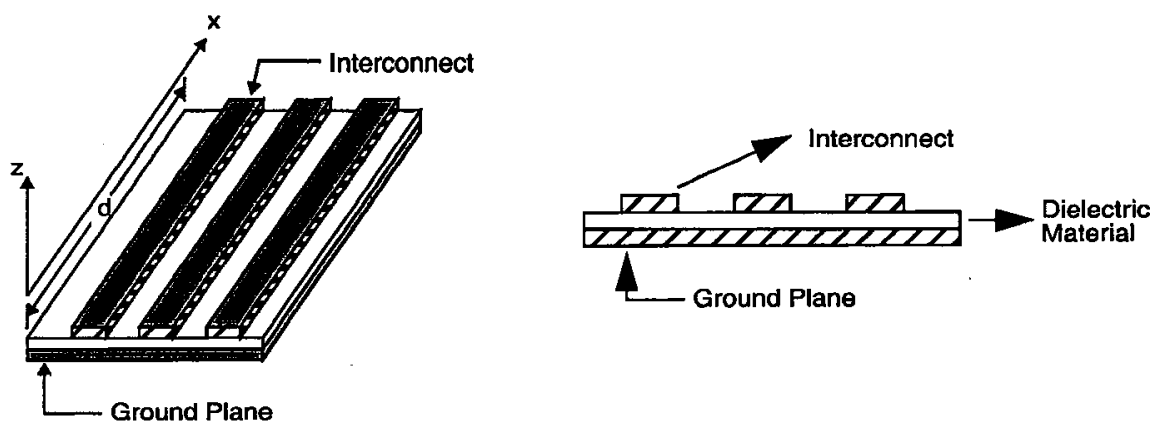

Figure 2.2 Top view and cross-sectional view of an interconnect system

\subsubsection{Quasi-Transverse Electromagnetic Models}

Transverse electromagnetic (TEM) waves exist for interconnects with homogeneous mediums and perfect conductors. Under these conditions interconnects produce electric and magnetic fields that are transverse (i.e. perpendicular to one another) and to the direction of propagation. Interconnects with inhomogeneous mediums produce electromagnetic waves with many velocities. As well, interconnects that have imperfect conductors produce an electric field along the surface conductor. Such structures violate the TEM characteristics, since TEM waves propagate with only one velocity and have no electric field along the surface conductor. Nevertheless, for most practical cases, the resulting field structure is similar to the TEM structure. Such field structures can still be approximated by TEM waves, which are more precisely referred to as quasi-TEM waves [2].

Quasi-TEM assumptions remain the dominant trend for analyzing lossy MTLs, since the approximation is valid for most practical interconnect structures and offers relative ease and low CPU cost compared to full wave approaches [2], [18]. The voltages and currents for the quasi-TEM distributed models are described by partial differential equations (PDEs) known as Telegrapher's equations. The simplest distributed model is the delay-line or lossless line. More complicated models include per-unit-length (p.u.l.) losses of the conductor and the dielectric substrate, as well as coupling between adjacent lines. At 
higher frequencies, edge, proximity, and skin effects become prominent and distributed models with frequency-dependent parameters are required. The difficulty with quasi-TEM distributed models is that they cannot be directly linked to circuit simulators such as SPICE. Circuit simulators solve nonlinear ordinary differential equations (ODEs) and have difficulty solving the PDEs. To overcome this difficulty, numerical techniques are used to convert distributed models into ODEs.

The simplest solution for linking distributed transmission lines to circuit simulators is to segment the line using the conventional lumped segmentation model [2]. The number of segments required depends on the electrical length of the transmission line. For transmission lines that are electrically long (i.e. the length of the line is much greater than the wavelength) many segments are required. In addition to the lumped segmentation model, other more sophisticated algorithms exist such as the method of characteristics [21]-[26] optimization techniques [27], Chebyshev polynomials [28]-[29] and wavelets [30]-[31]. In recent years, the development of passive macromodels has been a topic of intense research [13], [32]-[33]. Macromodels that are stable but not passive can produce unstable networks when connected to other passive loads. This can lead to spurious oscillations during transient analysis. Only passive macromodels can guarantee the stability of the overall network when terminated to other passive loads. Transmission line macromodels that are passive by construction are the integrated congruence transform [33], and compact finite difference technique [32].

\subsubsection{Full Wave Models}

If the cross-sectional dimensions of interconnects become a significant fraction of the circuit's operating wavelength, the field components in the direction of propagation can no longer be ignored [2]. Under these conditions, quasi-TEM assumptions become inadequate in describing interconnects and full wave models are required. These models are able to account for all possible field components and satisfy all boundary conditions required to accurately model the high-frequency effects of interconnects. 
Full wave analysis of interconnect structures have been successfully implemented using partial element equivalent circuit (PEEC) models [70]-[71]. PEEC models are lumped RLC elements that are extracted from the physical geometry using quasi-static solution of Maxwell's equations. Improved PEEC models include retardation effects (retarded PEEC or $\mathrm{rPEEC}$ ) and provide a full wave solution. These models usually produce large circuit networks and are very CPU intensive to solve.

\subsection{Frequency-Domain Analysis}

In this section, the frequency-domain solution of MTLs is presented. Consider a lossycoupled MTL containing $m$ signal conductors and one reference conductor as shown in Figure 2.3. The voltages and currents are functions of position $x$, and time $t$ and can be defined as vectors $v(x, t)$ and $i(x, t) \in \mathfrak{R}^{m}$, as

$$
\boldsymbol{v}(x, t)=\left[\begin{array}{c}
v_{1(x, t)} \\
\vdots \\
v_{m(x, t)}
\end{array}\right] \quad \boldsymbol{i}(x, t)=\left[\begin{array}{c}
i_{1(x, t)} \\
\vdots \\
i_{m(x, t)}
\end{array}\right]
$$

Under quasi-TEM distributed assumptions, the voltages and currents are related by Telegrapher's equations [2],

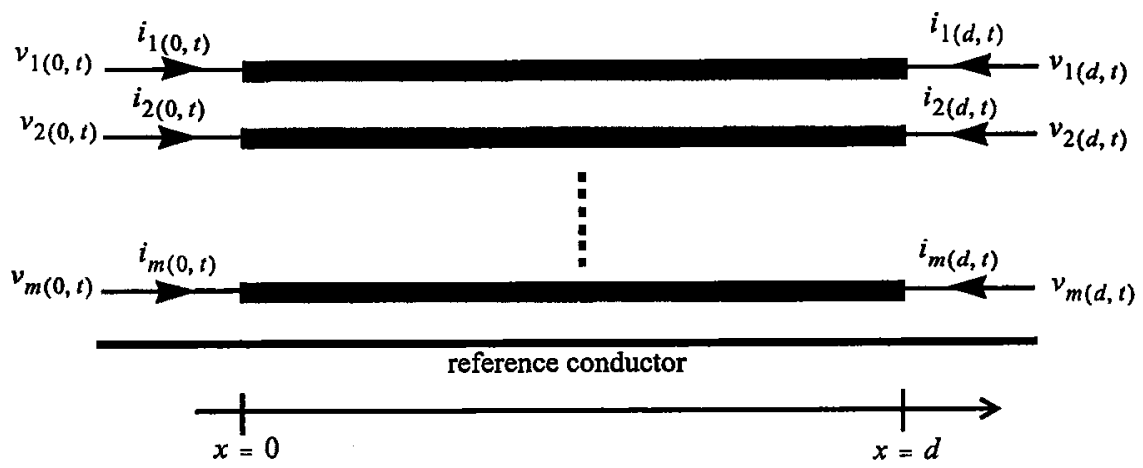

Figure 2.3 Multi-conductor transmission line 


$$
\begin{aligned}
& \frac{\partial}{\partial x} v(x, t)=-R i(x, t)-L \frac{\partial}{\partial t} i(x, t) \\
& \frac{\partial}{\partial x} i(x, t)=-G v(x, t)-C \frac{\partial}{\partial t} v(x, t)
\end{aligned}
$$

where $\boldsymbol{R} \in \mathfrak{R}^{m \times m}, \boldsymbol{L} \in \mathfrak{R}^{m \times m}, \boldsymbol{C} \in \mathfrak{R}^{m \times m}$ and $\boldsymbol{G} \in \mathfrak{R}^{m \times m}$ are the p.u.l. resistance, inductance, capacitance and conductance of the MTL, respectively. The Laplace domain representation of (2.2) becomes

$$
\begin{gathered}
\frac{\partial}{\partial x} V(x, s)=-Z I(x, s) \\
\frac{\partial}{\partial x} I(x, s)=-Y V(x, s)
\end{gathered}
$$

where $Z$ and $Y$ are the transmission line p.u.l. impedance and admittance parameters, defined as

$$
\boldsymbol{Z}=\boldsymbol{R}+s \boldsymbol{Y} \quad \boldsymbol{Y}=\boldsymbol{G}+\boldsymbol{C}
$$

and $s$ is the Laplace operator. Next, the frequency-domain solution of (2.3) is described using the eigen-decomposition approach and the matrix-exponential approach.

\subsubsection{Eigen-Decomposition Based Transmission Line Solution}

In this section, the transmission line solution of (2.3) is described in terms of eigen-decomposition. Differentiating (2.3) with respect to $x$, yields

$$
\begin{aligned}
& \frac{\partial^{2}}{\partial x^{2}} V(x, s)=-Z \frac{\partial}{\partial x} I(x, s) \\
& \frac{\partial^{2}}{\partial x^{2}} I(x, s)=-Y \frac{\partial}{\partial x} V(x, s)
\end{aligned}
$$

Substituting (2.3) into (2.5) forms a set of wave equations

$$
\begin{aligned}
\frac{\partial^{2}}{\partial x^{2}} V(x, s) & =Z Y V(x, s) \\
\frac{\partial^{2}}{\partial x^{2}} I(x, s) & =\boldsymbol{Y Z I}(x, s)
\end{aligned}
$$


The solution of the wave equations is based on decoupling the differential propagation modes of the coupled transmission lines [2]. For this purpose, eigen-decomposition is performed on the matrix $Z Y$, as

$$
Z Y S_{v}=S_{v} \Gamma_{m}^{2}
$$

where $S_{v}$ contains the right eigenvectors of $Z Y$ and $\Gamma_{m}^{2}$ is a diagonal matrix containing the eigenvalues $\gamma_{i}^{2}$ of $Z Y$,

$$
\Gamma_{m}^{2}=\left[\begin{array}{cccc}
\gamma_{1}^{2} & 0 & \ldots & 0 \\
0 & \gamma_{2}^{2} & \ldots & 0 \\
\vdots & \vdots & & \vdots \\
0 & 0 & \ldots & \gamma_{m}^{2}
\end{array}\right]
$$

For the general case of transmission lines in an inhomogeneous medium, the matrix $\boldsymbol{Z Y}$ contains $m$ distinct eigenvalues corresponding to the number of signal conductors. Using (2.8), the matrix $Z Y$ can be expressed as

$$
Z Y=S_{v} \Gamma_{m}^{2} S_{v}^{-1}
$$

Substituting (2.10) into (2.6) yields

$$
\frac{\partial^{2}}{\partial x^{2}} V(x, s)=S_{v} \Gamma_{m}^{2} S_{v}^{-1} V(x, s)
$$

To express (2.11) as a diagonal matrix, a variable substitution is used, where

$$
\tilde{V}(x, s)=S_{v}^{-1} V(x, s)
$$

Applying the variable substitution of (2.12) into (2.11) gives

$$
\frac{\partial^{2}}{\partial x^{2}} \tilde{V}(x, s)=\Gamma_{m}^{2} \tilde{V}(x, s)
$$

Equation (2.13) represents a set of decoupled second-order differential equations. The solution of (2.13) is similar to the scalar case and can be written in terms of forward and backward traveling waves as follows 


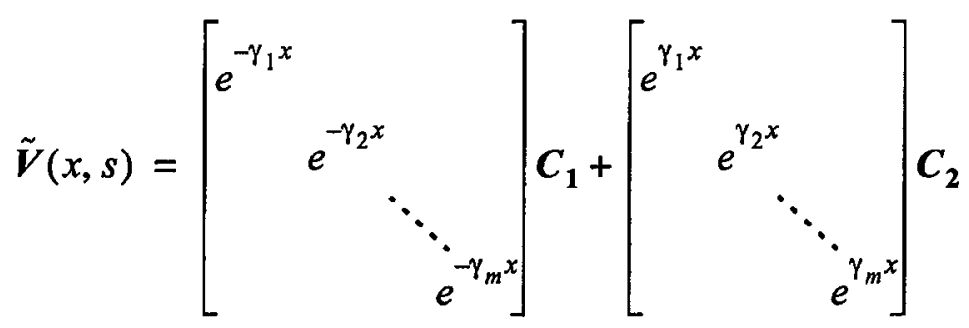

where $C_{1}$ and $C_{2}$ are constant vectors determined from the terminal currents and voltages at $x=0$ and $x=d$. Pre-multiplying (2.14) by $S_{v}$ and substituting (2.12) into (2.14), yields

$$
V(x, s)=S_{v} E(x) C_{1}+S_{v}[E(x)]^{-1} C_{2}
$$

where

$$
E(x)=\left[\begin{array}{lll}
e^{-\gamma_{1} x} & & \\
& e^{-\gamma_{2} x} & \\
& \ddots & \\
& & e^{-\gamma_{m} x}
\end{array}\right]
$$

From (2.15), the near and far end voltages at $x=0$ and $x=d$ respectively, can be expressed in matrix form as

$$
\left[\begin{array}{l}
V(0, s) \\
V(d, s)
\end{array}\right]=\left[\begin{array}{cc}
S_{v} & S_{v} \\
S_{v} E(d) & S_{v}[E(d)]^{-1}
\end{array}\right]\left[\begin{array}{l}
C_{1} \\
C_{2}
\end{array}\right]
$$

To obtain expressions for the currents, differentiating (2.15) with respect to $x$ and substituting in the first equation of (2.3), gives

$$
-S_{v} \Gamma_{m} E(x) C_{1}+S_{v} \Gamma_{m}[E(x)]^{-1} C_{2}=-Z I(x, s)
$$

Pre-multiplying (2.18) by $-Z^{-1}$, yields

$$
I(x, s)=S_{i} E(x) C_{1}-S_{i}[E(x)]^{-1} C_{2}
$$

where $S_{i}=Z^{-1} S_{\nu} \Gamma_{m}$. From (2.19), the near and far end currents at $x=0$ and $x=d$ respectively, are also expressed in matrix form as 


$$
\left[\begin{array}{c}
I(0, s) \\
-I(d, s)
\end{array}\right]=\left[\begin{array}{cc}
S_{i} & -S_{i} \\
S_{i} E(d) & -S_{i}[E(d)]^{-1}
\end{array}\right]\left[\begin{array}{l}
C_{1} \\
C_{2}
\end{array}\right]
$$

Note that the current vector $I(d, s)$ is negative and corresponds to the current direction of Figure 2.3. Using (2.17) and (2.20) to eliminate the constants $\boldsymbol{C}_{\mathbf{1}}$ and $\boldsymbol{C}_{\mathbf{2}}$, results in the following relationship between the voltages and currents

$$
\left[\begin{array}{c}
I(0, s) \\
-I(d, s)
\end{array}\right]=\left[\begin{array}{cc}
S_{i} & S_{i} \\
S_{i} E(d) & S_{i}[E(d)]^{-1}
\end{array}\right]\left[\begin{array}{cc}
S_{v} & S_{v} \\
S_{v} E(d) & S_{v}[E(d)]^{-1}
\end{array}\right]^{-1}\left[\begin{array}{l}
V(0, s) \\
V(d, s)
\end{array}\right]
$$

Simplifying (2.21), the Y-parameters of the transmission line can be expressed as

$$
\left[\begin{array}{l}
I(0, s) \\
I(d, s)
\end{array}\right]=\left[\begin{array}{ll}
Y_{11} & Y_{12} \\
Y_{21} & Y_{22}
\end{array}\right]\left[\begin{array}{l}
V(0, s) \\
V(d, s)
\end{array}\right]=\left[\begin{array}{ll}
S_{i} E_{1} S_{v}^{-1} & S_{i} E_{2} S_{v}^{-1} \\
S_{i} E_{2} S_{v}^{-1} & S_{i} E_{1} S_{v}^{-1}
\end{array}\right]\left[\begin{array}{l}
V(0, s) \\
V(d, s)
\end{array}\right]
$$

where

$$
\boldsymbol{E}_{1}=\operatorname{diag}\left\{\frac{1+e^{-2 \gamma_{i} d}}{1-e^{-2 \gamma_{i} d}}\right\} \quad \boldsymbol{E}_{2}=\operatorname{diag}\left\{\frac{-2 e^{-\gamma_{i} d}}{1-e^{-2 \gamma_{i} d}}\right\} \quad i=1,2, \ldots, m
$$

Equation (2.22) represents the solution of (2.2) in the frequency-domain. An alternative solution of the MTL equations is based on the matrix-exponential form, which is described in the next section.

\subsubsection{Matrix-Exponential Based Transmission Line Solution}

In this section, the transmission line solution of (2.3) is described in terms of a matrix-exponential. Equation (2.3) can be written in matrix form as,

$$
\frac{\partial}{\partial x}\left[\begin{array}{l}
V(x, s) \\
I(x, s)
\end{array}\right]=-(D+s E)\left[\begin{array}{l}
V(x, s) \\
I(x, s)
\end{array}\right]
$$

where

$$
D=\left[\begin{array}{ll}
0 & R \\
G & 0
\end{array}\right] \quad E=\left[\begin{array}{ll}
0 & L \\
C & 0
\end{array}\right]
$$


Using the terminal conditions, the solution of (2.24) can be written as

$$
\left[\begin{array}{c}
V(d, s) \\
-I(d, s)
\end{array}\right]=e^{-(D+s E) d}\left[\begin{array}{c}
V(0, s) \\
I(0, s)
\end{array}\right]
$$

Note that once again the current vector $I(d, s)$ is negative and corresponds to the current direction of Figure 2.3. The expression of (2.26) is in terms of the ABCD-parameters. It is possible to convert (2.26) in terms of the Y-parameters. Let

$$
T(s)=\left[\begin{array}{ll}
T_{11} & T_{12} \\
T_{21} & T_{22}
\end{array}\right]=e^{-(D+s E) d}
$$

Using some algebraic manipulations, the ABCD parameters of (2.26) can be expressed in terms of the Y-parameters as

$$
\left[\begin{array}{l}
I(0, s) \\
I(d, s)
\end{array}\right]=\left[\begin{array}{ll}
Y_{11} & Y_{12} \\
Y_{21} & Y_{22}
\end{array}\right]\left[\begin{array}{l}
V(0, s) \\
V(d, s)
\end{array}\right]=\left[\begin{array}{cc}
-T_{12}^{-1} T_{11} & T_{12}^{-1} \\
T_{22} T_{12}^{-1} T_{11}-T_{21} & -T_{22} T_{12}^{-1}
\end{array}\right]\left[\begin{array}{l}
V(0, s) \\
V(d, s)
\end{array}\right]
$$

Both Y-parameter solutions of (2.22) and (2.28) are equivalent. The next section describes the formulation of circuit and transmission line equations.

\subsection{Formulation of Circuit Equations}

Even though (2.22) and (2.28) represent the solution of the transmission line equations in the frequency-domain, it is difficult to use these expressions for time-domain analysis. To link interconnects with nonlinear circuit simulators, numerical methods are required to convert the transmission line equations into ODEs. Before proceeding with the description of transmission line simulation algorithms, it is useful to review the formulation of circuit equations.

\subsubsection{Linear Networks}

For both frequency and time-domain analysis, the first step is to formulate the modified nodal analysis (MNA) matrix [72]-[73]. For example, consider the linear circuit in Figure 2.4. Using Kirchoff's current law (KCL), the MNA equations in the time-domain are 


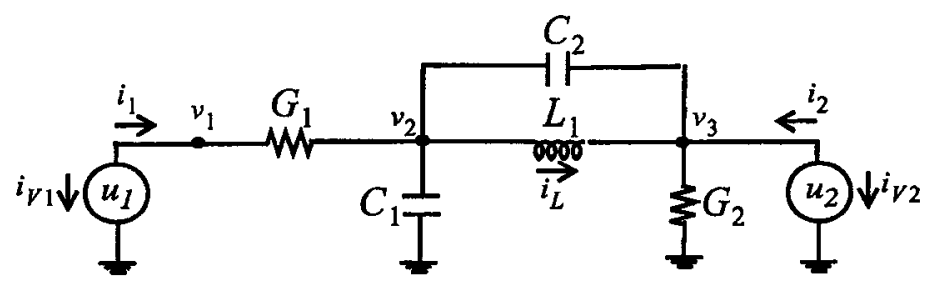

Figure 2.4 Linear circuit example

$$
\left[\begin{array}{lllllll}
0 & 0 & 0 & 0 & 0 & 0 \\
0 & \left(C_{1}+C_{2}\right) & -C_{2} & 0 & 0 & 0 \\
0 & -C_{2} & C_{2} & 0 & 0 & 0 \\
\hdashline 0 & 0 & 0 & L_{1} & 0 & 0 \\
0 & 0 & 0 & 0 & 0 & 0 \\
0 & 0 & 0 & 0 & 0 & 0
\end{array}\right]\left[\begin{array}{c}
\dot{v}_{1} \\
\dot{v}_{2} \\
\dot{v}_{3} \\
\dot{i}_{L} \\
\dot{i}_{V 1} \\
\dot{i}_{V 2}
\end{array}\right]+\left[\begin{array}{ccc:ccc}
G_{1} & -G_{1} & 0 & 0 & 1 & 0 \\
-G_{1} & G_{1} & 0 & 1 & 0 & 0 \\
0 & 0 & G_{2} & -1 & 0 & 1 \\
\hdashline 0 & -1 & 1 & 0 & 0 & 0 \\
-1 & 0 & 0 & 0 & 0 & 0 \\
0 & 0 & -1 & 0 & 0 & 0
\end{array}\right]\left[\begin{array}{c}
v_{1} \\
v_{2} \\
v_{3} \\
i_{L} \\
i_{V 1} \\
i_{V 2}
\end{array}\right]=\left[\begin{array}{cc}
0 & 0 \\
0 & 0 \\
0 & 0 \\
0 & 0 \\
-1 & 0 \\
0 & -1
\end{array}\right]\left[\begin{array}{l}
u_{1} \\
u_{2}
\end{array}\right]
$$

where $i_{L}$ is the inductance current; $i_{V 1}$ and $i_{V 2}$ are voltage source currents.

The MNA formulation of linear networks has the general form of (2.29) and can be written as

$$
\left[\begin{array}{ll}
\boldsymbol{Q} & 0 \\
\mathbf{0} & H
\end{array}\right]\left[\begin{array}{l}
\dot{v} \\
i
\end{array}\right]+\left[\begin{array}{cc}
N & E \\
-E^{T} & 0
\end{array}\right]\left[\begin{array}{l}
v \\
i
\end{array}\right]=B_{\phi} U_{\phi}
$$

or

$$
C_{\phi} \dot{x}_{\phi}(t)+G_{\phi} x_{\phi}(t)=b_{\phi}(t)
$$

where

$$
C_{\phi}=\left[\begin{array}{ll}
Q & 0 \\
0 & H
\end{array}\right] \quad G_{\phi}=\left[\begin{array}{cc}
N & E \\
-E^{T} & 0
\end{array}\right] \quad x_{\phi}(t)=\left[\begin{array}{l}
y \\
i
\end{array}\right] \quad b_{\phi}(t)=B_{\phi} U_{\phi}
$$

and

- $C_{\phi}, G_{\phi} \in \mathfrak{R}^{\phi \times \phi}$ are constant matrices describing the linear lumped memory and memoryless elements respectively. $\phi$ is the total number of variables in the circuit.

- $x_{\phi}(t) \in \mathfrak{R}^{\phi}$ is a vector of node voltage waveforms, appended by independent and 
dependent voltage source currents and linear inductor currents.

- $b_{\phi}(t) \in \Re^{\phi}$ is a vector with entries determined by independent voltage and current sources.

- $v$ and $i$ correspond to voltage and current variables, respectively.

- $N, \boldsymbol{Q}$ and $\boldsymbol{H}$ contain the stamps for the resistors, capacitors and inductors, respectively. $\boldsymbol{E}$ consists of ones, minus ones and zeros, which correspond to the current variables in the KCL equations. The superscript $T$ denotes the transpose of a matrix. $B_{\phi}$ is a selector matrix for the independent sources $\boldsymbol{U}_{\phi}$. Networks composed of passive linear elements, $N, \boldsymbol{Q}$ and $\boldsymbol{H}$ can be expressed as symmetric nonnegative definite matrices [13].

\subsubsection{Nonlinear Networks}

A simple example of a nonlinear network is shown in Figure 2.5. Using KCL, the MNA equations of Figure 2.5 can be written as

$$
\left[\begin{array}{llll}
0 & 0 & 0 & 0 \\
0 & 0 & 0 & 1 \\
0 & 0 & 0 & 0 \\
0 & 0 & 0 & 0
\end{array}\right]\left[\begin{array}{c}
\dot{v}_{1} \\
\dot{v}_{2} \\
\dot{i}_{V} \\
\dot{Q}
\end{array}\right]+\left[\begin{array}{llll}
0 & 0 & 1 & 0 \\
0 & G & 0 & 0 \\
1 & 0 & 0 & 0 \\
0 & 0 & 0 & 1
\end{array}\right]\left[\begin{array}{c}
v_{1} \\
v_{2} \\
i_{V} \\
Q
\end{array}\right]+\left[\begin{array}{c}
I_{s}\left(e^{\left(v_{1}-v_{2}\right) / v_{t}}-1\right) \\
-I_{s}\left(e^{\left(v_{1}-v_{2}\right) / v_{t}}-1\right) \\
0 \\
-k\left(v_{2}\right)^{2}
\end{array}\right]=\left[\begin{array}{c}
0 \\
0 \\
V(t) \\
0
\end{array}\right]
$$

where $i_{V}$ is the current voltage source and $Q$ is the charge on the nonlinear capacitor.

The general MNA formulation of nonlinear networks has the form of (2.33) and can be written as

$$
C_{\phi} \dot{x}_{\phi}(t)+G_{\phi} x_{\phi}(t)+f_{\phi}\left(x_{\phi}(t)\right)=b_{\phi}(t)
$$

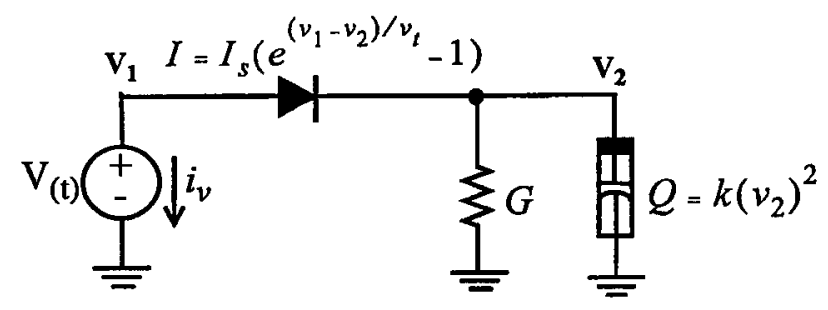

Figure 2.5 Rectifier circuit with nonlinear capacitor 
where

- $C_{\phi}, G_{\phi} \in \mathfrak{R}^{\phi \times \phi}$ are constant matrices describing the linear lumped memory and memoryless elements respectively. $\phi$ is the total number of variables in the circuit.

- $\boldsymbol{x}_{\phi}(t) \in \mathfrak{R}^{\phi}$ is the vector node voltage waveforms, appended by independent and dependent voltage source currents, linear inductor currents, nonlinear capacitor charge and nonlinear inductor flux waveforms.

- $\boldsymbol{f}_{\phi}\left(\boldsymbol{x}_{\phi}(t)\right)$ is a vector containing functions describing nonlinear circuit elements.

- $b_{\phi}(t) \in \mathfrak{R}^{\phi}$ is a vector with entries determined by independent voltage and current sources.

\subsubsection{Distributed Nonlinear Networks}

An example of a distributed nonlinear network is shown in Figure 2.6. The MNA equations of Figure 2.6 can be expressed as

$$
\left[\begin{array}{lll}
0 & 0 & 0 \\
0 & 0 & 1 \\
0 & 0 & 0
\end{array}\right]\left[\begin{array}{c}
\dot{v}_{1} \\
\dot{v}_{2} \\
\dot{Q}
\end{array}\right]+\left[\begin{array}{lll}
G & 0 & 0 \\
0 & 0 & 0 \\
0 & 0 & 1
\end{array}\right]\left[\begin{array}{l}
v_{1} \\
v_{2} \\
Q
\end{array}\right]+\left[\begin{array}{ll}
1 & 0 \\
0 & 1 \\
0 & 0
\end{array}\right]\left[\begin{array}{l}
i_{1} \\
i_{2}
\end{array}\right]+\left[\begin{array}{c}
0 \\
0 \\
-k\left(v_{2}\right)^{2}
\end{array}\right]=\left[\begin{array}{c}
I(t) \\
0 \\
0
\end{array}\right]
$$

where $Q$ is the charge of the nonlinear capacitor. The distributed transmission line can only be characterized in the frequency-domain by its terminal behavior as

$$
\left[\begin{array}{ll}
Y_{11} & Y_{12} \\
Y_{21} & Y_{22}
\end{array}\right]\left[\begin{array}{l}
V_{1} \\
V_{2}
\end{array}\right]=\left[\begin{array}{l}
I_{1} \\
I_{2}
\end{array}\right]
$$

where the Y-parameters of (2.36) are obtained from (2.22) or (2.28).

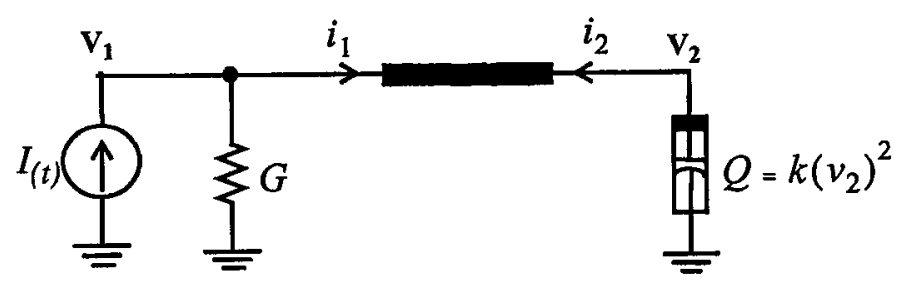

Figure 2.6 Distributed nonlinear circuit example 
The general MNA formulation of distributed nonlinear networks has the form of (2.35)-(2.36) and can be written as

$$
\begin{gathered}
C_{\phi} \dot{x}_{\phi}(t)+G_{\phi} x_{\phi}(t)+\sum_{k=1}^{N_{t}} D_{k} i_{k}(t)+f_{\phi}\left(x_{\phi}(t)\right)=b_{\phi}(t) \\
\boldsymbol{I}_{\boldsymbol{k}}(s)=\boldsymbol{Y}_{\boldsymbol{k}}(s) V_{\boldsymbol{k}}(s)
\end{gathered}
$$

where

- $C_{\phi}, G_{\phi} \in \mathfrak{R}^{\phi \times \phi}$ are constant matrices describing the linear lumped memory and memoryless elements respectively. $\phi$ is the total number of variables in the circuit.

- $\boldsymbol{x}_{\phi}(t) \in \mathfrak{R}^{\phi}$ is the vector node voltage waveforms, appended by independent and dependent voltage source currents, linear inductor currents, nonlinear capacitor charge and nonlinear inductor flux waveforms.

- $\boldsymbol{D}_{k}=\left[d_{i, j} \in\{0,1\}\right]$, is a selector matrix that maps $\boldsymbol{i}_{k}(t)$, the vector of terminal currents entering the interconnect subnetwork $k$, into the node space of the network, where $i \in\{1, \ldots, \phi\}, j \in\left\{1, \ldots, 2 m_{k}\right\}$ and $m_{k}+1$ is the number of conductors in subnetwork $k . N_{t}$ is the number of distributed structures. $\boldsymbol{Y}_{k}(s)$ is the admittance parameters of interconnect $k$ in the Laplace domain. $\boldsymbol{I}_{\boldsymbol{k}}$ and $\boldsymbol{V}_{\boldsymbol{k}}$ represent the Laplace terminal voltages and currents of interconnect $k$.

- $f_{\phi}\left(x_{\phi}(t)\right)$ is a vector containing functions describing nonlinear circuit elements.

- $b_{\phi}(t) \in \mathfrak{R}^{\phi}$ is a vector with entries determined by independent voltage and current sources.

There are two major difficulties in modeling distributed nonlinear networks in a circuit simulation environment. The first difficulty is due to the mixed frequency/time problem, which is encountered while linking distributed transmission lines with nonlinear circuit elements. This is because, distributed transmission lines are described by PDEs that are best solved in the frequency-domain, whereas nonlinear elements are described only in the time-domain as nonlinear ODEs. To overcome this difficulty, numerical methods are required to convert the transmission line equations into ODEs, so that (2.37) can be expressed as 


$$
C_{\pi} \dot{x}_{\pi}(t)+G_{\pi} x_{\pi}(t)+f_{\pi}\left(x_{\pi}(t)\right)=b_{\pi}(t)
$$

where the distributed elements are now explicitly embedded in the MNA matrices of the overall network. The second difficulty is due to the excessive CPU time associated with the simulation of transmission lines. For time-domain simulation, integration techniques are used to convert differential equations into difference equations [73]. To solve the nonlinear difference equations at each time point, Newton iterations are required. This causes the CPU cost to increase since the simulation of transmission line networks leads to large $\boldsymbol{C}_{\pi}$ and $\boldsymbol{G}_{\pi}$ matrices.

The goal of transmission line simulation algorithms is to address both the mixed frequency/time problem as well as reduce the CPU costs of the simulation. There are two simulation strategies for modeling transmission lines. One approach is based on developing efficient macromodels for transmission lines [18]-[33] and the other is based on MOR techniques [6]-[17]. In the following sections, several simulation strategies are examined to model transmission lines with nonlinear circuit elements.

\subsection{Simulation Techniques Based on Transmission Line Macromodels}

To simulate interconnects with nonlinear elements, macromodels are required to convert Telegrapher's equations into ODEs. These algorithms can be broadly classified into two main categories. One approach is based on developing macromodels for each transmission line network, the other is based on MOR of linear networks containing lumped and distributed elements.

An important issue in constructing macromodels is determining the bandwidth of interest. In most digital applications the bandwidth of interest is governed by the rise/fall time of the propagating signal. For instance, while a trapezoidal pulse has an energy spectrum spread over an infinite frequency range, most of the signal energy is concentrated near the low frequency region and decreases rapidly with an increase in frequency [7]. Hence, ignoring the high-frequency signal of the spectrum above a maximum frequency will not se- 
riously effect the overall signal shape. A useful relationship between the maximum frequency of interest $\left(f_{\text {max }}\right)$ and the rise/fall time of the signal $\left(t_{r}\right)$ is

$$
f_{\text {max }} \approx 0.35 / t_{r}
$$

which corresponds to the 3-dB bandwidth point of the energy spectrum [7], [19], [74]. For high-frequency applications, the maximum frequency can be more conservatively set to [7],

$$
f_{\text {max }} \approx 1 / t_{r}
$$

The following sections review some of the simulation algorithms that are used for macromodeling transmission lines.

\subsubsection{Conventional Lumped Segmentation}

This method uses lumped equivalent circuits to approximate (2.2). Applying Euler's method [2] to discretize (2.2) yields

$$
\begin{gathered}
v(x+x \Delta, t)-v(x, t)=-\Delta x R i(x, t)-\Delta x L \frac{\partial}{\partial t} i(x, t) \\
i(x+\Delta x, t)-i(x, t)=-\Delta x G v(x+\Delta x, t)-\Delta x C \frac{\partial}{\partial t} v(x+\Delta x, t)
\end{gathered}
$$

where $\Delta x=d / M$ is the length of each section, $d$ is the length of the total line and $M$ is the number of segments. A circuit representation of equation (2.41) is shown in Figure 2.7.

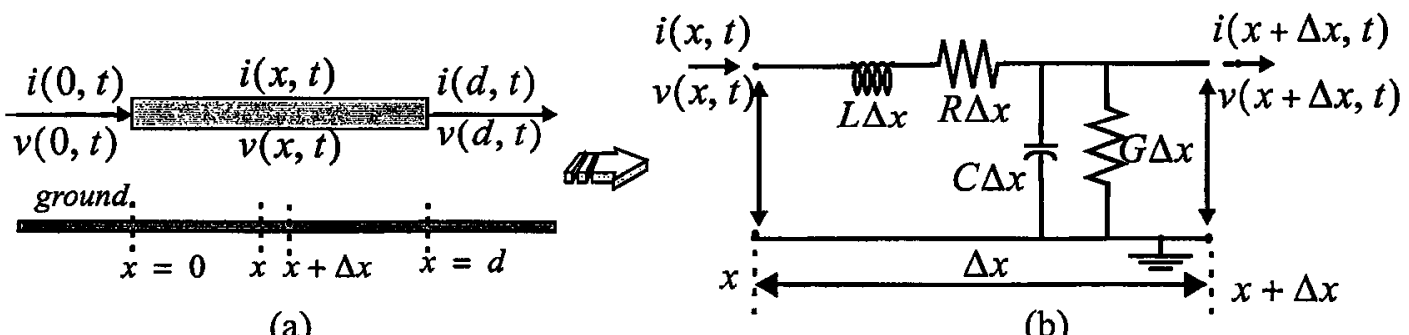

(a)

(b)

Figure 2.7 Lumped transmission line model 
The conventional lumped segmentation model provides a direct method to discretize interconnects, however, the approximation is only valid if $\Delta x$ is chosen to be a small fraction the wavelength. (i.e. $\lambda=v / f$, where $\lambda$ is the wavelength, $v$ is the velocity, $f$ is the frequency of interest). If the frequency of interest is high or if the interconnect is electrically long many lumped elements are required. This leads to large circuit matrices, rendering the method inefficient.

\subsubsection{Method of Characteristics}

The method of characteristics or Branin's method [21] is able to represent lossless interconnects as ODEs containing time delays. Although the original method of characteristics was developed in the time-domain using what is referred as characteristic curves (hence the name), a simpler alternative derivation in the frequency-domain is presented. The Laplace domain solution of (2.2) for two-conductor transmission line [5] is

$$
\begin{gathered}
{\left[\begin{array}{l}
I_{1} \\
I_{2}
\end{array}\right]=\frac{1}{Z_{0}\left(1-e^{-2 \gamma d}\right)}\left[\begin{array}{cc}
1+e^{-2 \gamma d} & -2 e^{-\gamma d} \\
-2 e^{-\gamma d} & 1+e^{-2 \gamma d}
\end{array}\right]\left[\begin{array}{l}
V_{1} \\
V_{2}
\end{array}\right]} \\
\gamma=\sqrt{(R+s L)(G+s C)} \\
Z_{0}=\sqrt{\frac{(R+s L)}{(G+s C)}}
\end{gathered}
$$

where $\gamma$ is the propagation constant and $Z_{0}$ is the characteristic impedance. The terms in (2.42) can be re-arranged to

$$
\begin{aligned}
& V_{1}=Z_{0} I_{1}+e^{-\gamma d}\left[2 V_{2}-e^{-\gamma d}\left(Z_{0} I_{1}+V_{1}\right)\right] \\
& V_{2}=Z_{0} I_{2}+e^{-\gamma d}\left[2 V_{1}-e^{-\gamma d}\left(Z_{0} I_{2}+V_{2}\right)\right]
\end{aligned}
$$

Defining $W_{1}$ and $W_{2}$ as

$$
\begin{aligned}
& W_{1}=e^{-\gamma d}\left[2 V_{2}-e^{-\gamma d}\left(Z_{0} I_{1}+V_{1}\right)\right] \\
& W_{2}=e^{-\gamma d}\left[2 V_{1}-e^{-\gamma d}\left(Z_{0} I_{2}+V_{2}\right)\right]
\end{aligned}
$$

Substituting (2.44) into (2.43) yields 


$$
\begin{aligned}
& V_{1}=Z_{0} I_{1}+W_{1} \\
& V_{2}=Z_{0} I_{2}+W_{2}
\end{aligned}
$$

The terms $Z_{0} I_{1}$ and $Z_{0} I_{2}$ in (2.45) are substituted in (2.44) to obtain

$$
\begin{aligned}
& W_{1}=e^{-\gamma d}\left[2 V_{2}-e^{-\gamma d}\left(2 V_{1}-W_{1}\right)\right] \\
& W_{2}=e^{-\gamma d}\left[2 V_{1}-e^{-\gamma d}\left(2 V_{2}-W_{2}\right)\right]
\end{aligned}
$$

Noting the symmetry of (2.46), a recursive relationship for $W_{1}$ and $W_{2}$ is obtained as

$$
\begin{aligned}
& W_{1}=e^{-\gamma d}\left[2 V_{2}-W_{2}\right] \\
& W_{2}=e^{-\gamma d}\left[2 V_{1}-W_{1}\right]
\end{aligned}
$$

If the transmission line is lossless, $\gamma$ and $Z_{0}$ are reduced to

$$
\gamma=s \sqrt{L C} \quad Z_{0}=\sqrt{\frac{L}{C}}
$$

This restriction makes $\gamma$ purely imaginary and $Z_{0}$ a real constant. The time-domain representation of (2.47) is obtained by taking the Laplace inverse, which replaces $e^{-\gamma d}$ by time shifts (or delays), expressed as

$$
\begin{aligned}
& w_{1}(t+\tau)=2 v_{2}(t)-w_{2}(t) \\
& w_{2}(t+\tau)=2 v_{1}(t)-w_{1}(t)
\end{aligned}
$$

A circuit representation of the transmission line can be obtained from (2.45) and (2.49) as shown in Figure 2.8.

For the case of lossy lines, $\gamma$ is not purely imaginary and $Z_{0}$ is not a real constant, hence (2.45) and (2.47) cannot be directly converted to the time-domain. The method of characteristics has been extended to model lossy transmission lines through Padé synthesis of the propagation constant and the characteristic impedance [22]-[23]. In case of MTLs, the method of characteristics is applied through decoupling of the transmission line equations [2], [24]-[26]. 


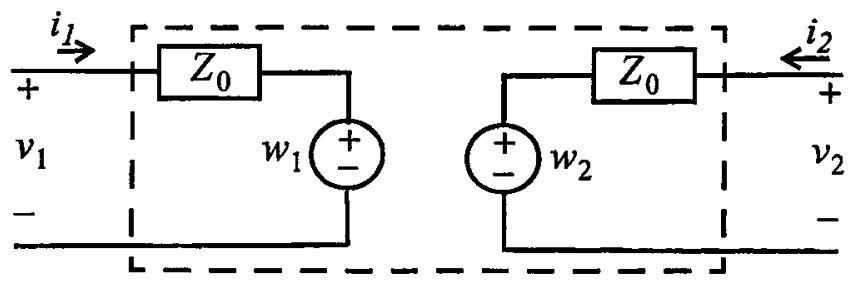

Figure 2.8 Method of characteristics transmission line model.

\subsubsection{Least Square Optimization}

Least square optimization techniques [27] derive transfer functions for the frequency response of transmission lines. The method fits sample data to a complex rational function as

$$
H(s)=c+\sum_{i=1}^{N} \frac{k_{i}}{s-p_{i}}
$$

where $c, k_{i}$ and $p_{i}$ represent the quotient, residues and poles, respectively. To obtain stable poles, the even part of $H(s)$ is fitted to the real data samples. The even part of $H(s)$ is expressed as

$$
\operatorname{Re}(H(s))=\frac{\sum_{i=0}^{N} a_{i}\left(\omega^{2}\right)^{i}}{1+\sum_{i=1}^{N} b_{i}\left(\omega^{2}\right)^{i}}
$$

where $s=j \omega$ and $\omega$ is the angular frequency. Writing (2.51) at the frequency points $\left[\omega_{0}, \omega_{1}, \ldots, \omega_{K}\right]$, yields the following set of linear equations

$$
\left[\begin{array}{ccccccc}
1 & \omega_{0}^{2} & \ldots & \omega_{0}^{2 N} & -\omega_{0}^{2} \operatorname{Re}\left(H\left(\omega_{0}\right)\right) & \ldots & -\omega_{0}^{2 N} \operatorname{Re}\left(H\left(\omega_{0}\right)\right) \\
1 & \omega_{1}^{2} & \ldots & \omega_{1}^{2 N} & -\omega_{1}^{2} \operatorname{Re}\left(H\left(\omega_{1}\right)\right) & \ldots & -\omega_{1}^{2 N} \operatorname{Re}\left(H\left(\omega_{1}\right)\right) \\
\vdots & \vdots & \vdots & \vdots & \vdots \\
1 & \omega_{K}^{2} & \ldots & \omega_{K}^{2 N} & -\omega_{K}^{2} \operatorname{Re}\left(H\left(\omega_{K}\right)\right) & \ldots & -\omega_{K}^{2 N} \operatorname{Re}\left(H\left(\omega_{K}\right)\right)
\end{array}\right]\left[\begin{array}{c}
a_{0} \\
\vdots \\
a_{N} \\
b_{1} \\
\vdots \\
b_{N}
\end{array}\right]=\left[\begin{array}{c}
\boldsymbol{R} \\
\operatorname{Re}\left(H\left(\omega_{0}\right)\right) \\
\vdots \\
\operatorname{Re}\left(H\left(\omega_{1}\right)\right) \\
\left.\left.\omega_{K}\right)\right)
\end{array}\right]
$$


The least square solution of (2.52) is

$$
\boldsymbol{A}^{T} \boldsymbol{A} \boldsymbol{x}=\boldsymbol{A}^{T} \boldsymbol{B}
$$

The poles of the system are obtained by computing the roots of the denominator of (2.51) and they belong to the left-hand-side plane only, since (2.51) is an even function. If any poles are purely imaginary, they are rejected. Next, the quotient and residues are obtained by matching the real and imaginary data to (2.50), as follows

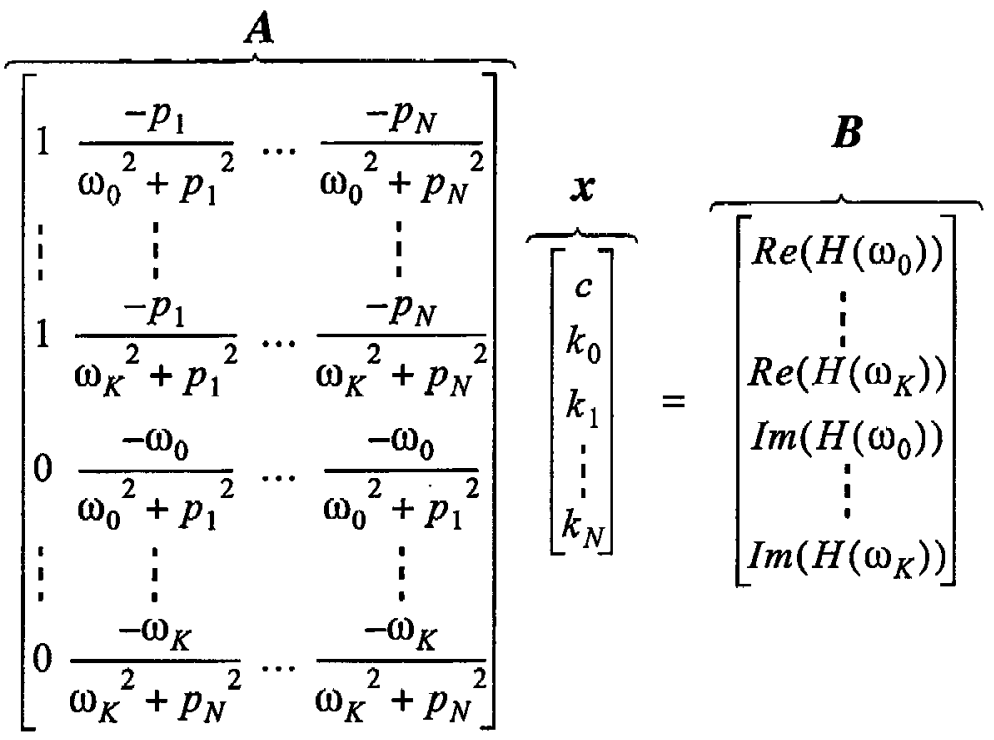

where the solution of (2.54) is obtained by solving (2.53). Once the coefficients of (2.50) are determined, the rational function can be described as ODEs.

Least square optimization provides great flexibility in modeling all types of interconnect configurations. However, the solution of (2.50) is ill conditioned. In addition, the algorithm does not guarantee the passivity of the macromodel by construction.

\subsubsection{Basis Function Macromodels}

This algorithm expresses the voltages and currents of transmission lines in terms of known basis functions such as Chebyshev polynomials [28]-[29] or wavelets [30]-[31]. For example, consider a two-conductor transmission line. The variations in space for the voltage $v(x, t)$ and current $i(x, t)$ can be expressed in terms of Chebyshev polynomials 
as

$$
v(x, t)=\sum_{n=0}^{N} a_{n}(t) T_{n}(x) \quad i(x, t)=\sum_{n=0}^{N} b_{n}(t) T_{n}(x)
$$

where $T_{n}(x)$ is the $n$th degree Chebyshev polynomial; $a_{n}(t)$ and $b_{n}(t)$ are the unknown variables. The derivatives of $v(x, t)$ and $i(x, t)$ with respect to $x$ are also expanded as Chebyshev polynomials

$$
\frac{\partial}{\partial x} v(x, t)=\sum_{n=0}^{N} \hat{a}_{n}(t) T_{n}(x) \quad \frac{\partial}{\partial x} i(x, t)=\sum_{n=0}^{N} \hat{b}_{n}(t) T_{n}(x)
$$

where $a_{n}(t), \hat{a}_{n}(t), b_{n}(t)$ and $\hat{b}_{n}(t)$ have the following relations [28],

$$
a_{n}(t)=\frac{1}{2 n}\left(\hat{a}_{n-1}(t)-\hat{a}_{n+1}(t)\right) \quad b_{n}(t)=\frac{1}{2 n}\left(\hat{b}_{n-1}(t)-\hat{b}_{n+1}(t)\right)
$$

Using (2.55)-(2.57) and the orthogonal properties of Chebyshev polynomials, Telegrapher's equations (2.2) can be converted to ODEs.

One advantage of the algorithm is that it is well suited to model non-uniform transmission lines by expanding the line parameters in terms of know basis functions with respect to position $x$ [28]-[31]. Chebyshev and wavelet models are able to achieve better accuracy with fewer variables when compared to the lumped segmentation model. However, the algorithm does not guarantee the passivity of the macromodel by construction.

\subsubsection{Compact Finite-Differences Based Approximation}

Compact differences can be used to convert Telegrapher's equations into ODEs [32]. Consider a two-conductor transmission line. The variations in space for the voltage $v(x, t)$ and current $i(x, t)$ can be expressed as

$$
V(x, s)=\sum_{m=1}^{P} V_{m}(s) A_{m}(x) ; \quad I(x, s)=\sum_{n=1}^{Q} I_{n}(s) B_{n}(x)
$$

where $A_{m}(x)$ and $B_{n}(x)$ are the known expansion functions and $I_{n}(s)$ and $V_{m}(s)$ are the unknown variables. It is assumed that the line is divided into $M$ equal segments of length 
$\Delta x$. The unknown voltage $V(x, s)$ can be expressed in terms of node values at $x=k \Delta x ;(k=0,1 \ldots M)$. The current $I(x, s)$ is expressed at the center of each segment as, $x=(k-1 / 2) \Delta x ;(k=0,1 \ldots M)$. The spatial derivatives of Telegrapher's equation are approximated using the following central difference operator as

$$
\left.\alpha_{1} \frac{\partial f(x)}{\partial x}\right|_{k+1}+\left.\alpha_{2} \frac{\partial f(x)}{\partial x}\right|_{k}+\left.\alpha_{1} \frac{\partial f(x)}{\partial x}\right|_{k-1}=\frac{f_{k+1 / 2}-f_{k-1 / 2}}{\Delta x}
$$

where $f(x)$ represents either $V(x)$ or $I(x)$, and $k$ denotes the index node. The coefficients $\alpha_{1}$ and $\alpha_{2}$ are selected to obtain the desired truncation error criteria. For example, fourth order approximation is achieved when $\alpha_{1}=1 / 24, \alpha_{2}=11 / 12$. Using (2.59) to discretize (2.2), yields

$$
\begin{array}{r}
-\alpha_{1} Z_{k+3 / 2} I_{k+3 / 2}-\alpha_{2} Z_{k+1 / 2} I_{k+1 / 2}-\alpha_{1} Z_{k-1 / 2} I_{k-1 / 2}=\frac{V_{k+1}-V_{k}}{\Delta x} \\
k=0,1, \ldots, M-2 \\
-\alpha_{1} Y_{k+1} V_{k+1}-\alpha_{2} Y_{k} V_{k}-\alpha_{1} Y_{k-1} V_{k-1}=\frac{I_{k+1 / 2}-I_{k-1 / 2}}{\Delta x} \\
k=0,1 \ldots M-1
\end{array}
$$

where $Z_{k}=R k \Delta x+s L k \Delta x$ and $Y_{k}=G k \Delta x+s C k \Delta x$. Using suitable compact difference operators for the terminal voltages and currents, $(2.60)$ can be expressed in terms of ODEs.

Compact difference algorithms can achieve better accuracy with fewer variables when compared to the lumped segmentation model. In addition, the algorithm is passive by construction.

\subsubsection{Integrated Congruence Transforms}

Integrated congruence transforms have been proposed for passive modeling of transmission lines [33]. Telegrapher's equations of (2.3) can be expressed in the frequency-domain 


$$
\left(s P+Q+T \frac{d}{d x}\right) \hat{Z}(x, s)=0
$$

where

$$
\hat{Z}(x, s)=\left[\begin{array}{l}
I(x, s) \\
V(x, s)
\end{array}\right] \quad P=\left[\begin{array}{ll}
L & 0 \\
0 & C
\end{array}\right] \quad Q=\left[\begin{array}{ll}
R & 0 \\
0 & G
\end{array}\right] \quad T=\left[\begin{array}{ll}
0 & 1 \\
1 & 0
\end{array}\right]
$$

and $I(x, s), V(x, s) \in \mathfrak{R}^{m}$ represent the current and voltage vectors as functions of position $x$ and Laplace operator $s$. Let the transformation be defined as

$$
\hat{Z}(x, s)=u(x) \times \hat{z}(s)
$$

where

$$
\left.u(x)\right|_{2 m \times n}=\left[\begin{array}{l}
\left.\boldsymbol{u}_{i}(x)\right|_{m \times n} \\
\left.\boldsymbol{u}_{v}(x)\right|_{m \times n}
\end{array}\right]=\left.\left[\begin{array}{lll}
\boldsymbol{u}_{i 1}(x) & \ldots & \boldsymbol{u}_{i n}(x) \\
\boldsymbol{u}_{v 1}(x) & \ldots & \boldsymbol{u}_{v n}(x)
\end{array}\right] \quad \hat{z}(s)\right|_{n \times 1}=\left[\begin{array}{c}
\hat{z}_{1}(s) \\
\ldots \\
\hat{z}_{n}(s)
\end{array}\right]
$$

Note that $\boldsymbol{u}(x)$ is a function of $x$ only, and not a function of $s$. Substituting (2.63) in (2.61), pre-multiplying by the transpose of $u(x)$ and integrating with respect to the normalized variable $x$ (from 0 to 1 ), yields

$$
(s \hat{\boldsymbol{P}}+\hat{\boldsymbol{Q}}+\hat{\boldsymbol{T}}) \hat{z}(s)=0
$$

where

$$
\hat{\boldsymbol{P}}=\int_{0}^{1} \boldsymbol{u}^{T}(x) \boldsymbol{P}(x) \boldsymbol{u}(x) d x \quad \hat{\boldsymbol{Q}}=\int_{0}^{1} \boldsymbol{u}^{T}(x) \boldsymbol{Q}(x) \boldsymbol{u}(x) d x \quad \hat{\boldsymbol{T}}=\int_{0}^{1} \boldsymbol{u}^{T}(x) \boldsymbol{T} \frac{d \boldsymbol{u}(x)}{d x}(x) d x
$$

Using some additional steps, (2.65) can be converted into ODEs. It can be shown that the integrated congruence transform macromodel is passive by construction [33].

\subsection{Simulation Techniques Based on Model Order Reduction}

MOR algorithms are able to capture the frequency response of large linear networks with 
low-order rational approximations. The underlying concept of MOR is that distributed networks usually have large number of poles, however, only a small percentage of these poles are dominant. Dominant pole are defined as poles that have significant influence in the behaviour of the network. By capturing only the dominant poles, the CPU expense of the simulation can be significantly reduced without compromising accuracy.

MOR techniques can be broadly classified into two main categories: approaches based on explicit moment matching based on direct Padé approximants [6]-[9], [16] and implicit moment matching based on projecting large matrices on its dominant eigenspace [10][14].

\subsubsection{Explicit Moment Matching}

Explicit moment matching techniques [6]-[9], [16] use direct Padé approximants to capture the frequency impulse response of networks. To illustrate this concept, consider a single-input single-output linear system and let $H(s)$ be the transfer function. Using a Maclaurin series expansion [6], $H(s)$ can be expressed as

$$
H(s) \approx m_{0}+m_{1} s+m_{2} s^{2}+\ldots
$$

where $m_{i}$ is referred to as the $i^{\text {th }}$ moment of $H(s)$. To construct a reduced-order macromodel, the series expansion of (2.67) is converted to a rational function using a Padé approximation as

$$
H(s) \approx m_{0}+m_{1} s+m_{2} s^{2}+\ldots+m_{N+L-1} s^{N+L-1} \approx \frac{a_{0}+a_{1} s+\ldots+a_{L} s^{L}}{1+b_{1} s+\ldots+b_{N} s^{N}}
$$

The coefficients $a_{i}$ and $b_{i}$ are obtained by cross-multiplying the denominator of (2.68) and equating similar powers of $s$, as 


$$
\begin{gathered}
{\left[\begin{array}{cccc}
m_{L-M+1} & m_{L-M+2} & \cdots & m_{L} \\
m_{L-M+2} & & \cdots & m_{L+1} \\
\vdots & \vdots & & \vdots \\
m_{L} & m_{L+1} & & m_{N+L-1}
\end{array}\right]\left[\begin{array}{c}
b_{N} \\
b_{N-1} \\
\vdots \\
b_{1}
\end{array}\right]=\left[\begin{array}{c}
m_{L+1} \\
m_{L+2} \\
\vdots \\
m_{L+N}
\end{array}\right]} \\
a_{0}=m_{0} \\
a_{1}=m_{1}+b_{1} m_{0} \\
\quad \ldots \\
a_{L}=m_{L}+\sum_{i=1}^{\min (L, N)} b_{i} m_{L-i}
\end{gathered}
$$

To obtain a reduced-order macromodel the moments of the network need to be evaluated. Consider the case of a linear lumped circuit of (2.31), written in the frequency-domain as

$$
\left(s C_{\phi}+G_{\phi}\right) X_{\phi}=B_{\phi} U_{\phi}
$$

Multiplying (2.71) by $\boldsymbol{G}_{\phi}^{-1}$, yields

$$
\left(I_{\phi}-s A_{\phi}\right) X_{\phi}=R_{\phi}
$$

where $I_{\phi}$ is the identity matrix and

$$
A_{\phi}=-G_{\phi}^{-1} C_{\phi} \quad R_{\phi}=G_{\phi}^{-1} B_{\phi} U_{\phi}
$$

Replacing $\boldsymbol{X}_{\phi}$ with a Maclaurin series expansion, gives

$$
\left(I_{\phi}-s A_{\phi}\right)\left(M_{0}+M_{1} s+M_{2} s^{2}+\ldots\right)=R_{\phi}
$$

Equating the coefficients of similar powers of $s$ and on both sides of (2.74), yields

$$
\begin{aligned}
M_{0} & =R_{\phi} \\
M_{i} & =-A_{\phi} M_{i-1} \quad i>0
\end{aligned}
$$

Equation (2.75) yields a closed form relationship for the computation of the moments $M_{i}$. For a particular output node of interest, the moments are converted to a rational function as described by (2.68)-(2.70). From (2.73) and (2.75), only one matrix inversion is re- 
quired (i.e one lower-upper (LU) decomposition [73]) to compute the moments. This significantly reduces the CPU cost, since the simulation of linear circuits require many LU decompositions.

Explicit moment matching techniques using a single Padé expansion is referred as asymptotic waveform evaluation (AWE) [6]-[7]. Usually, if the rational approximation contains more then eight poles, a single Padé expansion can produce inaccurate results since (2.69) becomes ill-conditioned. In addition, Padé approximants may produce unstable poles and provide no estimates for error bounds [3]. These problems have been addressed by the complex frequency hopping ( $\mathrm{CFH}$ ) [9] algorithm, which relies on multiple expansion points to construct a unified rational function. Another difficulty with explicit moment matching techniques is that there is no guarantee that the reduced order macromodel is passive.

\subsubsection{Implicit Moment Matching}

Implicit moment matching techniques use Krylov subspace approaches to project large matrices on its dominant eigenspace [3], [10]-[15]. To explain the basic concept of implicit moment matching techniques consider a linear lumped network described by (2.72). The main objective of Krylov subspace algorithms is to approximate $A_{\phi}$ with a reducedorder matrix that preserves the leading eigenvalues (those with the largest magnitude). This is accomplished by calculating the moments of the network to create the following matrix

$$
K=\left[\begin{array}{lllll}
M_{0} & M_{1} & M_{2} & \ldots & M_{q-1}
\end{array}\right]
$$

or using (2.75),

$$
K=\left[R_{\phi} R_{\phi} A_{\phi} R_{\phi} A_{\phi}^{2} \ldots R_{\phi} A_{\phi}^{q-1}\right]
$$

where $K \in \mathfrak{R}^{\phi x k}$ is a rectangular matrix. The variable $\phi$ corresponds to the size of the original network and $k$ is the number of columns in $K$ defined as $k=q \cdot N_{p}$, where $N_{p}$ is the number of ports. By making use of a numerically well conditioned algorithm known 
as the Arnoldi process [75], an orthonormal matrix $\boldsymbol{Q}$ is constructed from the column space of $K$, such that

$$
\begin{gathered}
\operatorname{colsp}(\boldsymbol{Q})=\operatorname{colsp}(\boldsymbol{K}) \\
\boldsymbol{Q}^{\boldsymbol{T}} \boldsymbol{Q}=\boldsymbol{I}_{k}
\end{gathered}
$$

where $I_{k}$ is the identity matrix. The reduced-order system is obtained by a change of variables in (2.71),

$$
X_{\phi}=Q Z_{k}
$$

where $Z_{k} \in \Re^{k}$ is the reduced order system variable. The reduced order system is obtained by congruent transformation by substituting (2.79) into (2.71) and pre-multiplying by $Q^{T}$, as

$$
\left(s \tilde{\boldsymbol{C}}_{k}+\tilde{\boldsymbol{G}}_{k}\right) \boldsymbol{Z}_{k}=\tilde{\boldsymbol{B}}_{k} \boldsymbol{U}_{\phi}
$$

where

$$
\tilde{\boldsymbol{G}}_{k}=\boldsymbol{Q}^{T} \boldsymbol{G}_{\phi} \boldsymbol{Q} \quad \tilde{\boldsymbol{C}}_{k}=\boldsymbol{Q}^{T} \boldsymbol{C}_{\phi} \boldsymbol{Q} \quad \tilde{\boldsymbol{B}}_{k}=\boldsymbol{Q}^{T} \boldsymbol{B}_{\phi}
$$

This reduces the size of the original network from $\phi$ unknowns to $k$ unknowns, where $k$ is generally much smaller than $\phi$.

The above MOR technique is known as the PRIMA algorithm [13]. One advantage of the PRIMA algorithm is that it is able to preserve the passivity of the reduced system. In addition, due to the numerical properties of the Arnoldi algorithm, implicit moment matching techniques are able to capture more poles from a single expansion than explicit moment matching techniques. However, while Krylov methods capture many poles, the reduced system may contain poles that are not dominant making the macromodel unnecessarily large compared to multi-point direct expansions such as CFH [14]-[16]. 


\section{Chapter 3}

\section{General Class of Passive Macromodels for High Speed Interconnects}

\subsection{Introduction}

In this chapter, a general class of passive macromodeling algorithm for multi-port distributed transmission lines is presented [46]-[52]. The proposed model is suitable for inclusion in general-purpose circuit simulators and overcomes the mixed frequency/time simulation difficulties encountered during the transient analysis. The transmission line model is based on a matrix rational approximation (MRA) used to approximate the solution of Telegrapher's equation. The key features of the algorithm are: the macromodel can be formulated analytically in terms of predetermined coefficients and the per-unit-length (p.u.1.) parameters; the passivity of the macromodel can be guaranteed by construction; the transmission line parameters can be lossy, coupled and frequency dependent.

The organization of this chapter is as follows. A review of the transmission line equations and the development of the MRA macromodel are described in section 3.2. Section 3.3 specifies the necessary conditions to ensure passivity for the proposed macromodel. Classes of rational approximations that guarantee passivity are described in 3.4. An error criterion for selecting the order of approximation is provided in section 3.5. 


\subsection{Development of the MRA Macromodel}

Transmission lines are described by a set of partial differential equations (PDEs) (2.2), which can be written in the frequency-domain as

$$
\begin{gathered}
\frac{\partial}{\partial x} V(x, s)=-(R+s L) I(x, s) \\
\frac{\partial}{\partial x} I(x, s)=-(G+s C) V(x, s)
\end{gathered}
$$

As shown in Chapter 2, the solution of (3.1) can be expressed as an exponential matrix function

$$
\left[\begin{array}{r}
V(d, s) \\
-I(d, s)
\end{array}\right]=e^{Z}\left[\begin{array}{c}
V(0, s) \\
I(0, s)
\end{array}\right]
$$

where

$$
\boldsymbol{Z}=\left[\begin{array}{cc}
0 & -\boldsymbol{a} \\
-\boldsymbol{b} & 0
\end{array}\right] \quad \boldsymbol{a}=(\boldsymbol{R}(s)+s L(s)) d \quad \boldsymbol{b}=(\boldsymbol{G}(s)+s \boldsymbol{C}(s)) d
$$

$V, I$ represent the Laplace domain terminal voltage and current vectors of the multi-conductor transmission line (MTL) and $d$ is the length of the line.

Equation (3.2) does not have a direct representation in the time-domain, which makes it difficult to interface with nonlinear simulators. The objective of this chapter is to present a general class of algorithm that can ensure passivity of any type of matrix rational approximation of (3.2).

A scalar exponential function can be approximated as a rational function as

$$
e^{x} \approx \frac{Q_{N}(x)}{P_{M}(x)}=\frac{\sum_{i=0}^{N} q_{i} x^{i}}{\sum_{i=0}^{M} p_{i} x^{i}}
$$

If the scalar $x$ is replaced by the matrix $Z$ then a rational matrix is obtained that can be used to model the transmission line network of (3.2) as 


$$
\boldsymbol{P}_{M}(\boldsymbol{Z})\left[\begin{array}{r}
\boldsymbol{V}(d, s) \\
-\boldsymbol{I}(d, s)
\end{array}\right] \approx \boldsymbol{Q}_{N}(\boldsymbol{Z})\left[\begin{array}{r}
\boldsymbol{V}(0, s) \\
\boldsymbol{I}(0, s)
\end{array}\right]
$$

where $\boldsymbol{P}_{M}(\boldsymbol{Z}), \boldsymbol{Q}_{N}(\boldsymbol{Z})$ are polynomial matrices expressed as

$$
\boldsymbol{Q}_{N}(\boldsymbol{Z})=\sum_{i=0}^{N} q_{i} Z^{i} ; \quad \boldsymbol{P}_{M}(\boldsymbol{Z})=\sum_{i=0}^{M} p_{i} Z^{i}
$$

Once the exponential matrix of (3.2) is represented as rational functions, ordinary differential equations (ODEs) can be obtained [46], [52]. The formulation of the macromodel is derived analytically in terms of predetermined coefficients (i.e. $p_{i}, q_{i}$ ) and the p.u.l. parameters of transmission lines. In the following section, the conditions to ensure passivity are described.

\subsection{Passivity Considerations}

One of the key issues of modeling transmission lines is the preservation of passivity. A passive macromodel implies that a network cannot generate more energy than it absorbs. Passivity is an important property to satisfy because stable, but not passive macromodels can produce unstable systems when connected to other passive loads [13]. The loss of passivity can be a serious problem for general circuit simulators because the overall network may encounter artificial oscillations.

A passivity proof for the MRA algorithm is described in [46], [76]. In this thesis an alternative proof is provided [49], since it is easier to prove passivity for different order rational approximations. In Chapter 6, different order rational approximations are used to obtain more accurate time-domain macromodels.

\subsubsection{Review of Passivity Properties}

A passive network implies that the overall network will remain asymptotically stable for any passive termination, however, a stable network does not imply passivity. A linear $\mathrm{n}$ port network with an admittance matrix $Y(s)$ is said to be passive for all signals of $z$, if 
and only if [77]-[79],

1. $\boldsymbol{Y}\left(s^{*}\right)=\boldsymbol{Y}^{*}(s)$ where $*$ is the complex conjugate operator.

2. $Y(s)$ is a positive real matrix. That is the product $z^{* T}\left[Y^{T}\left(s^{*}\right)+Y(s)\right] z \geq 0$ for all possible values of $s$ satisfying $\operatorname{Re}(s)>0$ and any arbitrary value of $z$. The superscript $T$ denotes the transpose of the matrix.

The first condition implies, that the coefficients of the rational function matrix generated by the proposed macromodel must be real. The second condition implies, that $Y(s)$ must be a positive real matrix for all $\operatorname{Re}(s)>0$, since the real part of $\boldsymbol{Y}(s)$ is $1 / 2\left[\boldsymbol{Y}^{T}\left(s^{*}\right)+\boldsymbol{Y}(s)\right]$. The coefficients generated by (3.5) are real values, therefore the first condition is always satisfied. The task that remains is to ensure that the rational approximation satisfies the second condition. For this purpose the following theorem is presented.

\subsubsection{Preserving Passivity}

To obtain a passive macromodel for (3.5), the following theorem is described.

\section{Theorem 3.1}

Let the rational function approximation of $e^{x}$ be

$$
e^{x} \approx \frac{Q_{N}(x)}{Q_{N}(-x)}=\frac{\sum_{i=0}^{N} q_{i} x^{i}}{\sum_{i=0}^{N} q_{i}(-x)^{i}}
$$

where the polynomial $Q_{N}(x)$ is strictly Hurwitz. If the above conditions are satisfied, then the rational matrix obtained by replacing the scalar $x$ with the matrix $Z$ of (3.3) results in a passive transmission line macromodel provided that $\boldsymbol{a}$ and $\boldsymbol{b}$ of (3.3) are positive real functions.

The form of the resulting matrix-rational approximation can be written as 


$$
Q_{N}(-Z)\left[\begin{array}{c}
V(d, s) \\
-I(d, s)
\end{array}\right] \approx Q_{N}(Z)\left[\begin{array}{c}
V(0, s) \\
I(0, s)
\end{array}\right]
$$

or

$$
\left[\begin{array}{ll}
Q_{N_{11}} & \boldsymbol{Q}_{N_{12}} \\
\boldsymbol{Q}_{N_{21}} & \boldsymbol{Q}_{N_{22}}
\end{array}\right]\left[\begin{array}{c}
\boldsymbol{V}(d, s) \\
-I(d, s)
\end{array}\right] \approx\left[\begin{array}{cc}
\boldsymbol{Q}_{N_{11}} & -\boldsymbol{Q}_{N_{12}} \\
-\boldsymbol{Q}_{N_{21}} & \boldsymbol{Q}_{N_{22}}
\end{array}\right]\left[\begin{array}{c}
\boldsymbol{V}(0, s) \\
\boldsymbol{I}(0, s)
\end{array}\right]
$$

where

$$
\begin{gathered}
\boldsymbol{Q}_{N}(\boldsymbol{Z})=\sum_{i=0}^{N} q_{i} \boldsymbol{Z}^{i} \\
\boldsymbol{Q}_{N_{11}}=\sum_{i=0}^{N} q_{i}\left[\frac{1}{2}\left(1+(-1)^{i}\right)(\boldsymbol{a b})^{i / 2}\right] \\
\boldsymbol{Q}_{N_{12}}=\sum_{i=0}^{N} q_{i}\left[\frac{1}{2}\left(1-(-1)^{i}\right)(\boldsymbol{a b})^{\frac{i-1}{2}} \boldsymbol{a}\right] \\
\boldsymbol{Q}_{N_{21}}=\sum_{i=0}^{N} q_{i}\left[\frac{1}{2}\left(1-(-1)^{i}\right)(\boldsymbol{b a})^{\frac{i-1}{2}} \boldsymbol{b}\right] \\
\boldsymbol{Q}_{N_{22}}=\sum_{i=0}^{N} q_{i}\left[\frac{1}{2}\left(1+(-1)^{i}\right)(\boldsymbol{b} \boldsymbol{a})^{i / 2}\right]
\end{gathered}
$$

The conditions on the scalar rational approximation represented by (3.7) in Theorem 3.1 essentially means the following: A strict Hurwitz polynomial (in this case $Q_{N}(x)$ ) has its roots only in the left half-plane. Hence the zeros of scalar rational approximation (3.7) are strictly on left-half plane. The poles of the rational approximation (3.7), which is given by the roots of $Q_{N}(-x)$ will all be on the right-half plane and are mirror images of zeros of (3.7). A graphical description of the pole-zero distribution requirement for the scalar rational approximation represented by (3.7) in Theorem 3.1 is shown in the Figure 3.1.

\section{Proof of Theorem-1}

The passivity proof associated with the above theorem is provided in terms of Y-parameter form of (3.9), written as 

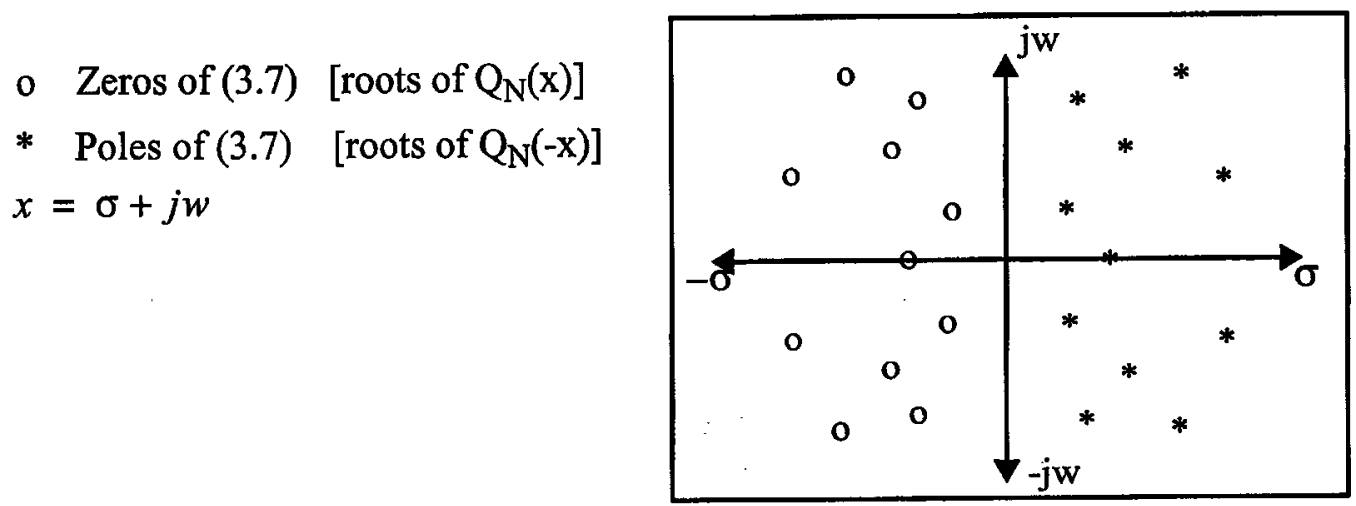

Figure 3.1 Pole-zero distribution for scalar rational approximation of (3.7)

$$
\left[\begin{array}{l}
I(0, s) \\
I(d, s)
\end{array}\right]=\left[\begin{array}{ll}
Y_{11} & Y_{12} \\
Y_{21} & Y_{22}
\end{array}\right]\left[\begin{array}{l}
V(0, s) \\
V(d, s)
\end{array}\right]
$$

where

$$
\left[\begin{array}{ll}
Y_{11} & Y_{12} \\
Y_{21} & Y_{22}
\end{array}\right]=\Psi_{1}+\Psi_{2}
$$

and

$$
\begin{gathered}
\Psi_{1}=\left[\begin{array}{ll}
\frac{1}{2}\left[Q_{N_{12}}\right]^{-1} Q_{N 11} & -\frac{1}{2}\left[Q_{N_{12}}\right]^{-1} Q_{N_{11}} \\
-\frac{1}{2}\left[Q_{N_{12}}\right]^{-1} Q_{N_{11}} & \frac{1}{2}\left[Q_{N_{12}}\right]^{-1} Q_{N_{11}}
\end{array}\right] \\
\Psi_{2}=\left[\begin{array}{ll}
\frac{1}{2}\left[Q_{N_{22}}\right]^{-1} Q_{N_{21}} & \frac{1}{2}\left[Q_{N_{22}}\right]^{-1} Q_{N_{21}} \\
\frac{1}{2}\left[Q_{N_{22}}\right]^{-1} Q_{N_{21}} & \frac{1}{2}\left[Q_{N_{22}}\right]^{-1} Q_{N_{21}}
\end{array}\right]
\end{gathered}
$$

To prove that $\Psi_{1}$ and $\Psi_{2}$ are positive real, the matrices are written in terms of congruent transforms as 


$$
\Psi_{1}=W_{\alpha}^{T}\left[\begin{array}{cc}
\frac{1}{2} H_{a} & 0 \\
0 & 0
\end{array}\right] W_{\alpha} \quad \Psi_{2}=W_{\beta}^{T}\left[\begin{array}{cc}
\frac{1}{2} H_{b} & 0 \\
0 & 0
\end{array}\right] W_{\beta}
$$

where

$$
\begin{array}{ll}
\boldsymbol{W}_{\alpha}=\left[\begin{array}{rr}
\boldsymbol{U} & -\boldsymbol{U} \\
\mathbf{0} & \boldsymbol{U}
\end{array}\right] & \boldsymbol{W}_{\boldsymbol{\beta}}=\left[\begin{array}{ll}
\boldsymbol{U} & \boldsymbol{U} \\
\mathbf{0} & \boldsymbol{U}
\end{array}\right] \\
\boldsymbol{H}_{a}=\left[\boldsymbol{Q}_{N_{12}}\right]^{-1} \boldsymbol{Q}_{N_{11}} & \boldsymbol{H}_{b}=\left[\boldsymbol{Q}_{N_{22}}\right]^{-1} \boldsymbol{Q}_{N_{21}}
\end{array}
$$

and $\boldsymbol{U}$ is the identity matrix.

If the matrices $H_{a}$ and $H_{b}$ are positive real, then $\Psi_{1}$ and $\Psi_{2}$ are positive real since they are expressed in terms of congruence transformations of positive real matrices (3.16). For this purpose, the condition that the polynomial $Q_{N}(x)$ of (3.7) is a strict Hurwitz polynomial is utilized. To identify if a polynomial is a strict Hurwitz polynomial, it is separated into even and odd parts [80] as

$$
\begin{gathered}
Q_{N}(x)=\sum_{i=0}^{N} q_{i} x^{i} \\
Q_{E V}(x)=\sum_{i=0}^{N} q_{i}\left[\frac{1}{2}\left(1+(-1)^{i}\right) x^{i}\right] \\
Q_{O D D}(x)=\sum_{i=0}^{N} q_{i}\left[\frac{1}{2}\left(1-(-1)^{i}\right) x^{i}\right]
\end{gathered}
$$

where $Q_{E V}(x)$ and $Q_{O D D}(x)$ represent the even and odd functions of $Q_{N}(x)$, respectively. The rational function formed from the even and odd polynomials can be expressed as a continued fraction expansion. For example, if the order of the polynomial is even, the continued fraction expansion becomes

$$
\frac{Q_{E V}(x)}{Q_{O D D}(x)}=\kappa_{N} x+\frac{1}{\kappa_{N-1} x+\frac{1}{\kappa_{N-2} x+\ldots}}
$$


The necessary and sufficient conditions for the polynomial $Q_{N}(x)$ to be a strict Hurwitz polynomial are the coefficients $\kappa_{i}$ for $i=1,2, \ldots, N$ must be strictly positive. This is stated in Lemma 3.1.

\section{Lemma 3.1}

- If $Q_{N}(x)$ is a strict Hurwitz polynomial, then the rational function created using the even and odd polynomials of (3.19) is an odd positive real rational function [80].

Also, the following facts are used to prove that $\boldsymbol{H}_{\boldsymbol{a}}$ and $\boldsymbol{H}_{b}$ are positive real.

\section{Lemma 3.2}

- The sum of two positive real matrices of similar dimensions is positive real [81].

\section{Lemma 3.3}

- The inverse of a positive real matrix (if the inverse exists), is positive real [81].

It should be noted that $Q_{E V}(x)$ and $Q_{O D D}(x)$ of (3.18) are very similar in form to $Q_{N_{11}}$, $\boldsymbol{Q}_{N_{12}}, \boldsymbol{Q}_{N_{21}}$ and $\boldsymbol{Q}_{N_{22}}$ of (3.11). In fact a relationship exists between the two forms and this relationship will be utilized to prove that $\boldsymbol{H}_{a}$ and $\boldsymbol{H}_{b}$ are positive real functions. Let the rational function formed by $Q_{E V}(x)$ and $Q_{O D D}(x)$ be expressed as a continued fraction:

$$
\begin{aligned}
& \frac{Q_{E V}(x)}{Q_{O D D}(x)}=\kappa_{N} x+\frac{1}{\kappa_{N-1} x+\frac{1}{\kappa_{N-2} x+{ }}} \quad \text { if } N=\text { Even } \\
& \kappa_{N-2} x+\ldots \\
& \because \mathrm{\kappa}_{1} x \\
& \frac{Q_{E V}(x)}{Q_{O D D}(x)}=\frac{1}{\kappa_{N} x+\frac{1}{\kappa_{N-1} x+\frac{1}{\kappa_{N-2}+\ldots}}} \quad \text { if } N=O d d \\
& \kappa_{N-2} x+\ldots \\
& \because{ }^{\prime}{ }_{1} x
\end{aligned}
$$


Since $Q_{N}(x)$ is a Hurwitz polynomial, the coefficients $\kappa_{i} i=[1 \ldots N]$ are all positive values and the rational functions in (3.20) are positive real (from lemma 3.1). Next, $\boldsymbol{H}_{a}$ can also be expressed as a continued fraction expansion as

$$
\begin{array}{ll}
\boldsymbol{H}_{a}=\kappa_{N} \boldsymbol{b}+\left(\kappa_{N-1} \boldsymbol{a}+\left(\ldots+\left(\kappa_{2} b+\left(\kappa_{1} a\right)^{-1}\right)^{-1}\right)^{-1}\right)^{-1} & \text { if } N=\text { Even } \\
\boldsymbol{H}_{\boldsymbol{a}}=\left(\kappa_{N} \boldsymbol{a}+\left(\kappa_{N-1} b+\left(\ldots+\left(\kappa_{2} b+\left(\kappa_{1} a\right)^{-1}\right)^{-1}\right)^{-1}\right)^{-1}\right)^{-1} & \text { if } N=\text { Odd }
\end{array}
$$

The coefficients $\kappa_{i} i=[1 \ldots N]$ in (3.21) are the same as in (3.20). Since theorem 3.1 assumes $\mathbf{a}$ and $\mathbf{b}$ are positive real matrices, $\boldsymbol{H}_{a}$ in (3.21) is also positive-real (from lemma 3.2 and lemma 3.3).

Next, to prove that $\boldsymbol{H}_{b}$ is positive real a similar strategy is followed. Let the rational function formed by $Q_{E V}(x)$ and $Q_{O D D}(x)$ be expressed as

$$
\begin{aligned}
& \frac{Q_{O D D}(x)}{Q_{E V}(x)}=\kappa_{N} x+\frac{1}{\kappa_{N-1} x+\frac{1}{\kappa_{N-2} x+\ldots}} \quad \text { if } N=O d d \\
& \because{ }_{\mathrm{K}_{1} x} \\
& \frac{Q_{O D D}(x)}{Q_{E V}(x)}=\frac{1}{\kappa_{N} x+\frac{1}{\kappa_{N-1} x+\frac{1}{\kappa_{N-2} x+\ldots}}} \quad \text { if } N=\text { Even }
\end{aligned}
$$

Since $Q_{N}(x)$ is a Hurwitz polynomial, the coefficients $\kappa_{i} i=[1 \ldots N]$ are all positive values and the rational functions in (3.22) are positive real (from lemma 3.1). Next, $\boldsymbol{H}_{b}$ can also be expressed as a continued fraction expansion as 


$$
\begin{array}{ll}
\boldsymbol{H}_{b}=\kappa_{N} b+\left(\kappa_{N-1} a+\left(\ldots+\left(\kappa_{2} a+\left(\kappa_{1} b\right)^{-1}\right)^{-1}\right)^{-1}\right)^{-1} & \text { if } N=\text { Odd } \\
\boldsymbol{H}_{b}=\left(\kappa_{N} a+\left(\kappa_{N-1} b+\left(\ldots+\left(\kappa_{2} a+\left(\kappa_{1} b\right)^{-1}\right)^{-1}\right)^{-1}\right)^{-1}\right)^{-1} & \text { if } N=\text { Even }
\end{array}
$$

The coefficients $\kappa_{i} i=[1 \ldots N]$ in (3.23) are the same as in (3.22). Since $\mathbf{a}$ and $\mathbf{b}$ are positive real matrices, $\boldsymbol{H}_{b}$ in (3.23) is also positive-real (from lemma 3.2 and lemma 3.3). This completes the proof of Theorem 3.1.

It should be noted that for the case of frequency-independent p.u.l. parameters, $\mathbf{a}$ and $\mathbf{b}$ are always positive real matrices due to the fact that the line parameters $R, L, G$ and $C$ are nonnegative matrices [2], [23]. For the case of frequency-dependent p.u.l. parameters, the functions are also positive real, however, the parameters are often described by tabulated data obtained from measurements, empirical formulae or electromagnetic simulation [2], [82]-[87]. Thus to obtain a passive macromodel, it is required to fit the frequency-dependent parameters to positive real functions. A scheme for obtaining positive real rational functions from tabulated data is described in chapter 5 .

\subsection{Classes of Rational Approximations}

Any rational function approximation that satisfies the conditions of Theorem 3.1 can be used to obtain passive macromodels for transmission lines. In this section, two types of rational approximations for the exponential function are examined. The first method uses a Padé approximation for the predetermined coefficients of (3.7). The second method uses near optimal coefficients for (3.7).

\subsubsection{Padé Rational Approximation}

One method to obtain the predetermined coefficients of (3.7), is to use a Padé rational function [46]. The Padé coefficients of $e^{x}$ can be obtained using the following closedform relation [73], 


$$
e^{x}=\frac{\sum_{i=0}^{M} \frac{(M+N-i) ! M !}{(M+N) ! i !(M-i) !} x^{i}}{\sum_{i=0}^{N} \frac{(M+N-i) ! N !}{(M+N) ! i !(N-i) !}(-x)^{i}}
$$

For the case when $M=N$, the form of (3.24) is that of (3.7) and the Hurwitz condition is satisfied [88].

\subsubsection{Minimax Rational Approximation}

A compact expression for (3.7) can be obtained using a minimax approximation [49]. The goal is to minimize the error function for a given interval, such that

$$
\max _{\left[0, \omega_{m a x}\right]} W(\omega)\left|e^{j \omega}-\frac{Q_{N}(j \omega)}{Q_{N}(-j \omega)}\right| \quad \text { is minimum }
$$

where $W(\omega)$ is a given weight function, $\left[0, \omega_{\max }\right]$ is the interval of approximation, $Q(j \omega)$ and $Q(-j \omega)$ are the polynomials of the rational function, $N$ is the order of approximation and $s=j \omega$.

Let the rational function of $e^{s}$ be expressed in terms of products of factors written as

$$
\begin{array}{ll}
e^{s} \approx \frac{Q_{N}(s)}{Q_{N}(-s)}=\frac{\prod_{i=1}^{N / 2}\left(s^{2}+\kappa_{1, i} s+\kappa_{0, i}\right)}{\prod_{i=1}^{N / 2}\left(s^{2}-\kappa_{1, i} s+\kappa_{0, i}\right)} & N=E v e n \\
e^{s} \approx \frac{Q_{N}(s)}{Q_{N}(-s)}=\frac{\left(s+\kappa_{0,0}\right) \prod_{i=1}^{(N-1) / 2}\left(s^{2}+\kappa_{1, i} s+\kappa_{0, i}\right)}{\left(-s+\kappa_{0,0}\right) \prod_{i=1}^{(N-1) / 2}\left(s^{2}-\kappa_{1, i} s+\kappa_{0, i}\right)} & N=\text { Odd }
\end{array}
$$

Equations (3.26) and (3.27) satisfies the form of (3.7). Imposing the constraints that $\kappa_{0, i}, \kappa_{1, i}>0$ for all values of $N$ will ensure that $Q_{N}(s)$ is strict Hurwitz polynomial. Re- 
placing $s=j \omega$ and separating the rational function in terms of real and imaginary parts, the minimax objective function can be written as

$$
\max \left(W _ { r e ^ { ( \omega _ { i } ) } } \left(\cos \omega_{i}-\operatorname{Re}\left(\frac{Q_{N^{\left(\omega_{k}\right)}}}{\left.Q_{N^{\left(-\omega_{k}\right)}}\right)}\right)^{2}+W_{i m^{\left(\omega_{i}\right)}}\left(\sin \omega_{i}-\operatorname{Im}\left(\frac{Q_{N^{\left(\omega_{k}\right)}}}{\left.Q_{N^{\left(-\omega_{k}\right)}}\right)}\right)^{2}\right)\right.\right. \text { is minimum }
$$

such that $\kappa_{0, i}, \kappa_{1, i}>0$ for all values of $i$. The variables $W_{r e}\left(\omega_{i}\right), W_{i m}\left(_{i}\right)$ are the weight functions at the angular frequency $\omega_{k}$, where $\omega_{k}$ ranges from $0 \leq \omega_{1}<\ldots<\omega_{k} \leq \omega_{\text {max }}$; $\operatorname{Re}(), \operatorname{Im}()$ are the real and imaginary parts of the rational function.

It should be emphasized that the minimax optimization is performed on SCALAR functions of $e^{s}$ and is independent of the number of coupled lines and the p.u.l. parameters. The results obtained are then stored and the macromodel can be obtained analytically in terms of the predetermined coefficients and p.u.l. parameters of the transmission line.

\subsection{Criteria for Selecting the Order of Approximation}

As mentioned in the previous chapter, the bandwidth of interest is determined by the rise/ fall time of the propagating signal. A useful relationship between the maximum frequency $\left(f_{\max }\right)$ and the rise/fall time of the signal $\left(t_{r}\right)$ is provided by $(2.39)$ and $(2.40)$. These equations have the following form

$$
f_{\max } \approx K_{0} / t_{r}
$$

where $K_{0}$ is real constant value. If $K_{0}=0.35$ then the frequency $f_{\max }$ corresponds to the 3-db bandwidth point, however, for high frequency applications it is recommended to use a more conservative estimate by setting $K_{0}=1$ [7]. To obtain accurate macromodels for general interconnect networks using the MRA algorithm, it is recommended to use conservative estimates (i.e. $K_{0}$ can range between 0.6 to 1 to obtain accurate macromodels).

Accuracy of the proposed model depends on the order of the approximation. Since all the elements in (3.8) are computed using the pre-determined coefficients and in a closedform manner, the required order $N$ can be easily estimated using the following error criterion. 


$$
\left\|e^{Z}-\left(Q_{N}^{-1}(-Z) Q_{N}(Z)\right)\right\|<\varepsilon
$$

where $\varepsilon$ is the pre-defined error tolerance. If the error tolerance is not satisfied over the frequency range of interest, the order of the macromodel can be increased. 


\section{Chapter 4}

\section{Development of Time-Domain}

\section{Macromodel}

\subsection{Introduction}

In this chapter, a technique to link the matrix rational approximation (MRA) algorithm to nonlinear circuit simulators is described [46], [52]. Time-domain macromodels are derived for RC, lossless and lossy (RLGC) parameter models. In addition, the application of the MRA is extended to include incident field coupling on multi-conductor transmission lines (MTLs) [64]-[65], [89]. There are many ways to convert rational functions into ordinary differential equations. A direct approach is to represent rational functions in terms of state-space equations, either in the controllable, observable or Jordan canonical form [90][91]. An alternative approach is to use circuit elements. One advantage of using circuit elements is that the macromodel can be realized without having knowledge or access to the internals of the circuit simulator. In addition, circuit realization methods can lead to diagonally dominant, band limited, and highly sparse matrices.

The formulation of the time-domain macromodel is given in section 4.2. Section 4.3 describes a technique to realize the MRA in terms of circuit elements. Application of the MRA to include incident field coupling on MTLs is described in section 4.4. Numerical examples showing the validity of the MRA are provided in section 4.5. 


\subsection{Development of Time-Domain Macromodel}

In this section, a technique to realize the MRA algorithm in terms of circuit elements is presented. The formulation of the macromodel is described in terms of predetermined coefficients and the per-unit-length (p.u.1.) parameters.

\subsubsection{Formulation of Circuit Equations}

As described in Chapter 2, transmission lines in the presence of nonlinear elements can be written as

$$
\begin{gathered}
C_{\phi} \dot{x}_{\phi}(t)+G_{\phi} x_{\phi}(t)+D i(t)+f_{\phi}\left(x_{\phi}(t)\right)=b_{\phi}(t) \\
I(s)=\boldsymbol{Y}(s) V(s)
\end{gathered}
$$

where the matrices of (4.1) are defined in section 2.4.3. For clarity, and without loss of generality, (4.1) contains one transmission line network. The distributed network of (4.1) does not have a direct representation in the time-domain, leading to mixed frequency/time simulation difficulty. In order to overcome this problem, the MRA algorithm is used to convert (4.1) to

$$
C_{\pi} \frac{d}{d t} x_{\pi}(t)+G_{\pi} x_{\pi}(t)+f_{\pi}\left(x_{\pi}(t)\right)=b_{\pi}(t)
$$

where the distributed elements are now explicitly embedded in the modified nodal analysis (MNA) matrices of the overall network and

$$
G_{\pi}=G_{a}+G_{T} \quad C_{\pi}=C_{a^{+}} C_{T}
$$

Here the matrices $\boldsymbol{G}_{\boldsymbol{a}}, \boldsymbol{C}_{\boldsymbol{a}}, \boldsymbol{x}_{\pi}(t), \boldsymbol{f}_{\pi}\left(\boldsymbol{x}_{\pi}(t)\right)$ and $\boldsymbol{b}_{\pi}(t)$ are obtained from $\boldsymbol{G}_{\phi}, \boldsymbol{C}_{\phi}$, $x_{\phi}(t), f_{\phi}\left(x_{\phi}(t)\right)$ and $b_{\phi}(t)$ by appending their rows and/or columns that contain zeros to account for the extra variables required for the stamp of the transmission line. Thus, $\boldsymbol{G}_{a}$, $C_{a}, x_{\pi}(t), f_{\pi}\left(x_{\pi}(t)\right)$ and $b_{\pi}(t)$ can be expressed in the following block form

$\boldsymbol{G}_{a}=\left[\begin{array}{cc}\boldsymbol{G}_{\phi} & 0 \\ \mathbf{0} & 0\end{array}\right] \quad \boldsymbol{C}_{a}=\left[\begin{array}{cc}\boldsymbol{C}_{\phi} & 0 \\ \mathbf{0} & 0\end{array}\right] \quad \boldsymbol{x}_{\pi}(t)=\left[\begin{array}{c}\boldsymbol{x}_{\phi}(t) \\ \boldsymbol{x}_{T}(t)\end{array}\right] \boldsymbol{f}_{\pi}\left(\boldsymbol{x}_{\pi}(t)\right)=\left[\begin{array}{c}\boldsymbol{f}_{\phi}\left(\boldsymbol{x}_{\phi}(t)\right) \\ \mathbf{0}\end{array}\right] \boldsymbol{b}_{\pi}(t)=\left[\begin{array}{c}\boldsymbol{b}_{\phi}(t) \\ \mathbf{0}\end{array}\right]$ 
where $\boldsymbol{x}_{T}(t)$ are the variables introduced by the transmission line macromodel. The matrices $G_{T}$ and $C_{T}$ correspond to the stamp of the transmission line and are described in greater detail in the following section, which describes the time-domain formulation of the MRA algorithm.

\subsubsection{Formulation of MRA Macromodel}

The time-domain macromodel is derived by expressing the MRA in terms of products of low order rational functions, which can be realized in terms of circuit elements. The formulation of the macromodel is derived from the predetermined coefficients of the scalar approximation of $e^{x}$ and the p.u.l. parameters.

The solution of the transmission line equations can be expressed as in (3.2),

$$
\left[\begin{array}{c}
\boldsymbol{V}(d, s) \\
-\boldsymbol{I}(d, s)
\end{array}\right]=e^{\boldsymbol{Z}}\left[\begin{array}{c}
\boldsymbol{V}(0, s) \\
\boldsymbol{I}(0, s)
\end{array}\right]
$$

where

$$
\boldsymbol{Z}=\left[\begin{array}{cc}
\mathbf{0} & -\boldsymbol{a} \\
-\boldsymbol{b} & 0
\end{array}\right] \quad \boldsymbol{a}=(\boldsymbol{R}(s)+s L(s)) d \quad \boldsymbol{b}=(\boldsymbol{G}(s)+s C(s)) d
$$

The MRA algorithm relies on the scalar approximation of $e^{x}$ to model (4.5). To preserve passivity, the scalar approximation of (3.7) must satisfy the conditions of theorem 3.1. To realize the MRA macromodel in terms of circuit elements, (3.7) is expressed in terms of poles and zeros as

$$
\begin{aligned}
& e^{x} \approx \frac{Q_{N}(x)}{Q_{N}(-x)}=\frac{\sum_{i=0}^{N} q_{i} x^{i}}{\sum_{i=0}^{N} q_{i}(-x)^{i}}=\frac{\prod_{i=1}^{N / 2}\left[\left(\alpha_{i}+x\right)\left(\alpha_{i}^{*}+x\right)\right]}{\prod_{i=1}^{N / 2}\left[\left(\alpha_{i}-x\right)\left(\alpha_{i}^{*}-x\right)\right]} ; \quad \text { if } N \text { is even } \\
& e^{x} \approx \frac{Q_{N}(x)}{Q_{N}(-x)}=\frac{\sum_{i=0}^{N} q_{i} x^{i}}{\sum_{i=0}^{N} q_{i}(-x)^{i}}=\frac{\left(\alpha_{0}+x\right) \prod_{i=1}^{(N-1) / 2}\left[\left(\alpha_{i}+x\right)\left(\alpha_{i}^{*}+x\right)\right]}{\left(\alpha_{0}-x\right) \prod_{i=1}^{(N-1) / 2}\left[\left(\alpha_{i}-x\right)\left(\alpha_{i}^{*}-x\right)\right]} ; \quad \text { if } N \text { is odd }
\end{aligned}
$$


where $\alpha_{i}=x_{i}+j y_{i}$ are complex roots for $i>0, \alpha_{0}$ is a real root and $*$ is the complex conjugate operator. If the matrix $Z$ of (4.6) is used to replace the scalar $x$, then the MRA macromodel to approximate (4.5) becomes

$$
\left[\begin{array}{c}
V(d, s) \\
-I(d, s)
\end{array}\right] \approx\left[Q_{N}(-Z)\right]^{-1} Q_{N}(Z)\left[\begin{array}{l}
V(0, s) \\
I(0, s)
\end{array}\right]
$$

where $\left[Q_{N}(-Z)\right]^{-1} Q_{N}(Z)$ can also be expressed in terms of products of subsections as

$$
\begin{gathered}
\left.\boldsymbol{Q}_{N}(-Z)\right]^{-1} \boldsymbol{Q}_{N}(Z)=\prod_{i=1}^{N / 2}\left[\left(\alpha_{i} U_{-} Z\right)\left(\alpha_{i}^{*} U_{-} Z\right)\right]^{-1}\left[\left(\alpha_{i} U_{+} Z\right)\left(\alpha_{i}^{*} U_{+} Z\right)\right] \quad \text { if } N \text { is even } \\
{\left[\boldsymbol{Q}_{N}(-Z)\right]^{-1} \boldsymbol{Q}_{N}(Z)=\left[\alpha_{0} U_{-} Z\right]^{-1}\left[\alpha_{0} U_{+} Z\right] \cdot} \\
\prod_{i=1}^{(N-1) / 2}\left[\left(\alpha_{i} U_{-} Z\right)\left(\alpha_{i}^{*} U_{-} Z\right)\right]^{-1}\left[\left(\alpha_{i} U_{+} Z\right)\left(\alpha_{i}^{*} U_{+} Z\right)\right] \quad \text { if } N \text { is odd }
\end{gathered}
$$

where $\boldsymbol{U}$ is an identity matrix and $\alpha_{0}, \alpha_{i}=x_{i}+j y_{i}$ and $\alpha_{i}^{*}=x_{i}-j y_{i}$ are obtained from the scalar approximation of (4.7). The representation of (4.9) expresses the macromodel as series of subsections, where each $i^{\text {th }}$ subsection can be written as

$$
\left[\begin{array}{c}
\boldsymbol{V}_{i+1} \\
-\boldsymbol{I}_{i+1}
\end{array}\right]=\left[\boldsymbol{Q}_{N}(-\boldsymbol{Z})\right]_{i}^{-1}\left[\boldsymbol{Q}_{N}(\boldsymbol{Z})\right]_{i}\left[\begin{array}{c}
\boldsymbol{V}_{i} \\
\boldsymbol{I}_{i}
\end{array}\right]
$$

A graphical representation of (4.9) is given in Figure 4.1, where $V(0, s), I(0, s), V(d, s)$ and $I(d, s)$ represent the terminal voltages and currents. $V_{1} \ldots V_{i}$ and $I_{1} \ldots I_{i}$ represent the internal voltages and currents created by the MRA macromodel. Each subsection consists of two complex pole-zero pairs or one real pole-zero pair derived from the scalar approximation of (4.7). The matrices $\left[\boldsymbol{Q}_{N}(-\boldsymbol{Z})\right]_{i}$ and $\left[\boldsymbol{Q}_{N}(\boldsymbol{Z})\right]_{i}$ are given by

$$
\begin{aligned}
& {\left[\boldsymbol{Q}_{N}(-Z)\right]_{i}=\left[\left(\alpha_{i} \boldsymbol{U}-Z\right)\left(\alpha_{i}^{*} \boldsymbol{U}-Z\right)\right]=\left[\begin{array}{cc}
\boldsymbol{a b}+\left(x_{i}^{2}+y_{i}^{2}\right) \boldsymbol{U} & 2 x_{i} \boldsymbol{a} \\
2 x_{i} \boldsymbol{b} & \boldsymbol{a b}+\left(x_{i}^{2}+y_{i}^{2}\right) \boldsymbol{U}
\end{array}\right]} \\
& {\left[\boldsymbol{Q}_{N}(Z)\right]_{i}=\left[\left(\alpha_{i} U+Z\right)\left(\alpha_{i}^{*} \boldsymbol{U}+\boldsymbol{Z}\right)\right]=\left[\begin{array}{cc}
\boldsymbol{a b}+\left(x_{i}^{2}+y_{i}^{2}\right) \boldsymbol{U} & -2 x_{i} \boldsymbol{a} \\
-2 x_{i} \boldsymbol{b} & \boldsymbol{a b}+\left(x_{i}^{2}+y_{i}^{2}\right) \boldsymbol{U}
\end{array}\right]}
\end{aligned}
$$


for the subsection consisting of complex pole-zero pairs and

$$
\begin{gathered}
{\left[Q_{N}(-Z)\right]_{0}=\left[\alpha_{0} U-Z\right]=\left[\begin{array}{cc}
\alpha_{0} U & a \\
b & \alpha_{0} U
\end{array}\right]} \\
{\left[Q_{N}(Z)\right]_{0}=\left[\alpha_{0} U+Z\right]=\left[\begin{array}{cc}
\alpha_{0} U & -a \\
-b & \alpha_{0} U
\end{array}\right]}
\end{gathered}
$$

for the subsection consisting of a real pole-zero pair. Converting each subsection of (4.10) to the Y-parameters yields the circuit realization of the macromodel. The Y-parameters for the complex pole-zero pairs (4.11) are

$$
\begin{aligned}
& \boldsymbol{Y}_{11}=\boldsymbol{Y}_{22}=\frac{\left(x_{i}^{2}+y_{i}^{2}\right)}{4 x_{i}} a^{-1}+\frac{1}{4 x_{i}} \boldsymbol{b}+x_{i}\left(\boldsymbol{a}+\left(x_{i}^{2}+y_{i}^{2}\right) \boldsymbol{b}^{-1}\right)^{-1} \\
& \boldsymbol{Y}_{12}=\boldsymbol{Y}_{21}=\frac{-\left(x_{i}^{2}+y_{i}^{2}\right)}{4 x_{i}} a^{-1}-\frac{1}{4 x_{i}} \boldsymbol{b}+x_{i}\left(\boldsymbol{a}+\left(x_{i}^{2}+y_{i}^{2}\right) \boldsymbol{b}^{-1}\right)^{-1}
\end{aligned}
$$

and the Y-parameters for the real pole-zero pair (4.12) are

$$
\begin{aligned}
& Y_{11}=Y_{22}=\frac{\alpha_{0}}{2} a^{-1}+\frac{1}{2 \alpha_{0}} b \\
& Y_{12}=Y_{21}=-\frac{\alpha_{0}}{2} a^{-1}+\frac{1}{2 \alpha_{0}} b
\end{aligned}
$$

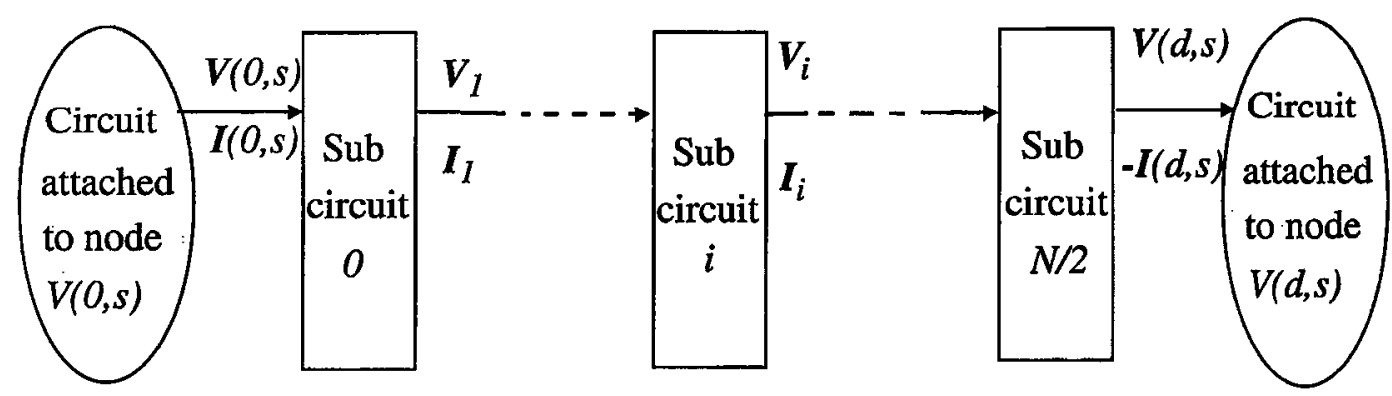

Figure 4.1 MRA macromodel represented in terms of the subsections of (4.9) 
The subsections of (4.13) and (4.14) are realized in terms of circuit elements and are assembled together to form the MNA matrices $G_{T}$ and $C_{T}$ of (4.3), where

$$
\boldsymbol{G}_{\boldsymbol{T}}=\sum_{i}\left(\psi_{i}\right)^{\mathbf{T}} \boldsymbol{G}_{i} \psi_{i} \quad \boldsymbol{C}_{\boldsymbol{T}}=\sum_{i}\left(\psi_{i}\right)^{\mathbf{T}} \boldsymbol{C}_{i} \psi_{i}
$$

The matrices $G_{i}$ and $C_{i}$ are derived from the Y-parameters of (4.13) and (4.14) and $\psi_{i}$ is a selector matrix that maps the block matrices $G_{i}$ and $C_{i}$ to the rest of the network equations. In the following section, the matrices $G_{i}$ and $C_{i}$ are derived for the $\mathrm{RC}$, lossless and lossy (RLGC) transmission line models.

\subsection{Circuit Realization of MRA Macromodel}

In this section, the time-domain macromodel is derived for the $\mathrm{RC}$, lossless and lossy (RLGC) transmission line models. The method realizes the Y-parameters of (4.13) and (4.14) in terms of circuit elements to obtain the $G_{i}$ and $C_{i}$ matrices of (4.15). Circuit representations are provided for the two-conductor and MTLs.

\subsubsection{RC Transmission Lines}

For RC transmission lines [51], the Y-parameters of (4.13) and (4.14) reduce to

$$
\begin{aligned}
& \boldsymbol{Y}_{11}=\boldsymbol{Y}_{22}=\frac{\left(x_{i}^{2}+y_{i}^{2}\right)}{4 x_{i}}(\boldsymbol{R} d)^{-1}+\frac{1}{4 x_{i}} s C d+x_{i}\left(\boldsymbol{R} d+\left(x_{i}^{2}+y_{i}^{2}\right)(s C d)^{-1}\right)^{-1} \\
& Y_{12}=Y_{21}=\frac{-\left(x_{i}^{2}+y_{i}^{2}\right)}{4 x_{i}}(\boldsymbol{R} d)^{-1}-\frac{1}{4 x_{i}} s C d+x_{i}\left(\boldsymbol{R} d+\left(x_{i}^{2}+y_{i}^{2}\right)(s C d)^{-1}\right)^{-1}
\end{aligned}
$$

for the complex pole-zero pairs and

$$
\begin{aligned}
& Y_{11}=Y_{22}=\frac{\alpha_{0}}{2}(R d)^{-1}+\frac{1}{2 \alpha_{0}} s C d \\
& Y_{12}=Y_{21}=-\frac{\alpha_{0}}{2}(R d)^{-1}+\frac{1}{2 \alpha_{0}} s C d
\end{aligned}
$$

for the real pole-zero pair. Circuit equivalent models that represent the Y-parameters of (4.16) and (4.17) are shown in Figure 4.2 for a two-conductor RC transmission line. Fig- 
ure 4.2a shows the topology corresponding to the complex pole-zero subsection of (4.16) and Figure $4.2 \mathrm{~b}$ shows the topology for the real pole-zero subsection of (4.17). The approach outlined in this section can be extended to MTLs. An example realization of a three-conductor RC transmission line is shown in Figure 4.3. The MNA matrices of each subsection $G_{i}$ and $C_{i}$ of (4.15) are

$$
\boldsymbol{G}_{i}=\left[\begin{array}{ccc}
\left(\frac{x_{i}}{d}+\frac{x_{i}^{2}+y_{i}^{2}}{4 x_{i} d}\right) R^{-1} & \frac{-x_{i}}{d} R^{-1} & -\left(\frac{x_{i}^{2}+y_{i}^{2}}{4 x_{i} d}\right) R^{-1} \\
\frac{-x_{i}}{d} R^{-1} & \frac{x_{i}}{d} R^{-1} & 0 \\
-\left(\frac{x_{i}^{2}+y_{i}^{2}}{4 x_{i} d}\right) R^{-1} & 0 & \left(\frac{x_{i}^{2}+y_{i}^{2}}{4 x_{i} d}\right) R^{-1}
\end{array}\right] \quad C_{i}=\left[\begin{array}{ccc}
\frac{d}{4 x_{i}} C & 0 & \frac{-d}{4 x_{i}} C \\
0 & \frac{x_{i} d}{x_{i}^{2}+y_{i}^{2}} C & \frac{x_{i} d}{x_{i}^{2}+y_{i}^{2}} \boldsymbol{C} \\
\frac{-d}{4 x_{i}} C \frac{x_{i} d}{x_{i}^{2}+y_{i}^{2}} C\left(\frac{d}{4 x_{i}}+\frac{x_{i} d}{x_{i}^{2}+y_{i}^{2}}\right) C
\end{array}\right]
$$

for the complex pole-zero pairs and

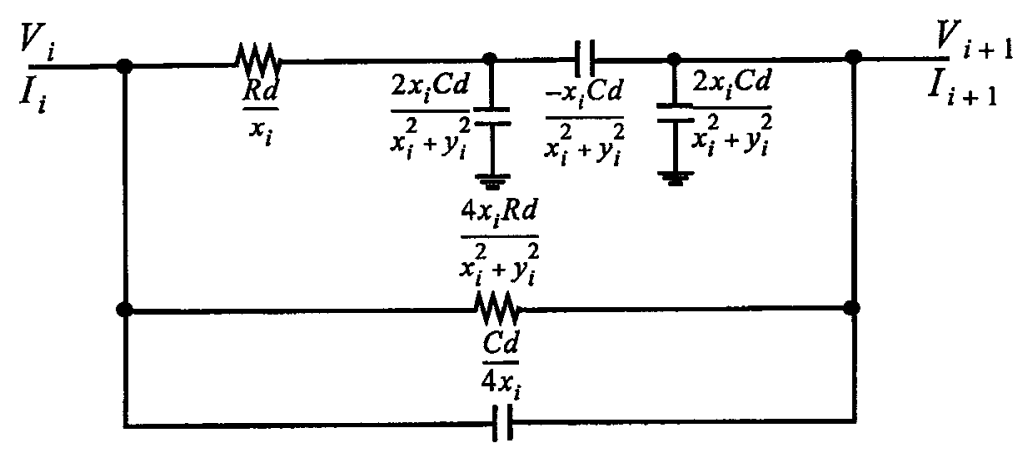

a) Complex pole-zero subsection

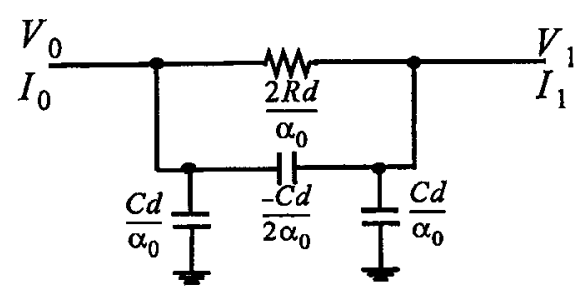

b) Real pole-zero subsection

Figure 4.2 Realization of two-conductor RC transmission line 


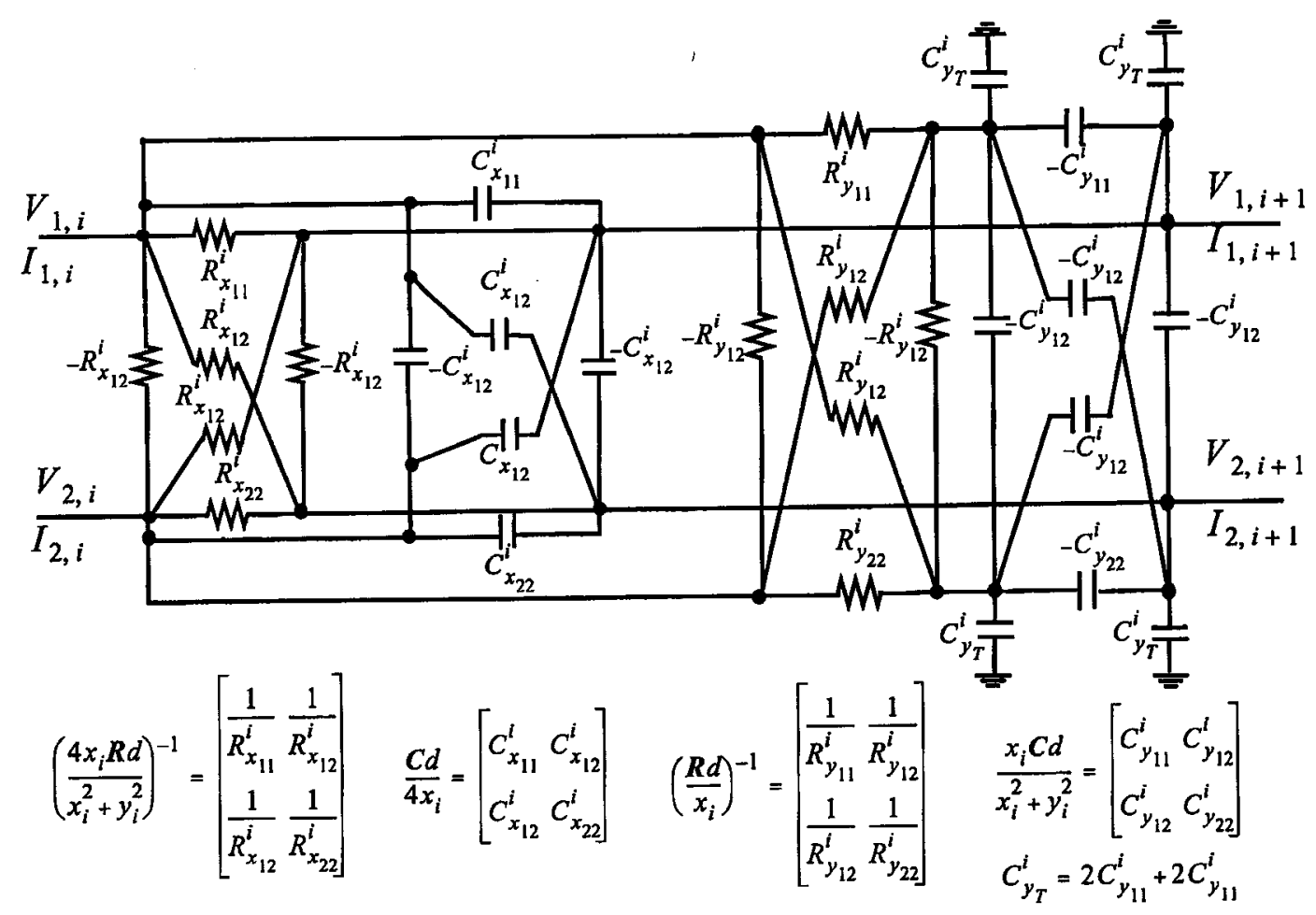

a) Complex pole-zero subsection

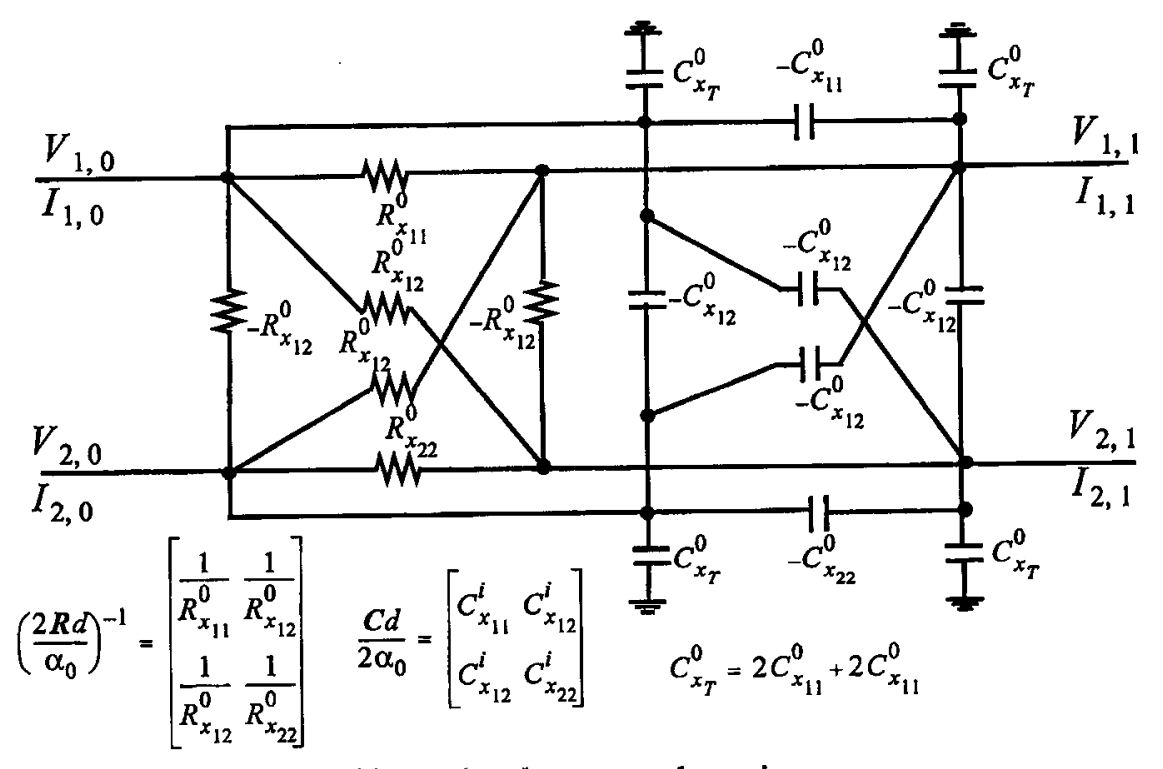

b) Real pole-zero subsection

Figure 4.3 Realization of RC MTL 


$$
G_{0}=\left[\begin{array}{ll}
\frac{\alpha_{0}}{2 d} R^{-1} & \frac{-\alpha_{0}}{2 d} R^{-1} \\
\frac{-\alpha_{0}}{2 d} R^{-1} & \frac{\alpha_{0}}{2 d} R^{-1}
\end{array}\right] \quad C_{0}=\left[\begin{array}{ll}
\frac{d}{2 \alpha_{0}} C & \frac{d}{2 \alpha_{0}} C \\
\frac{d}{2 \alpha_{0}} C & \frac{d}{2 \alpha_{0}} C
\end{array}\right]
$$

for the real pole-zero pairs. Equations (4.18) and (4.19) are equivalent to Figure 4.2 and Figure 4.3 and correspond to the MNA stamp of each subsection for RC transmission lines.

\subsubsection{Lossless Transmission Lines}

For the case of lossless transmission lines, the Y-parameters of (4.13) and (4.14) reduce to

$$
\begin{aligned}
& \boldsymbol{Y}_{11}=\boldsymbol{Y}_{22}=\frac{\left(x_{i}^{2}+y_{i}^{2}\right)}{4 x_{i}}(s L d)^{-1}+\frac{1}{4 x_{i}} s C d+x_{i}\left(s L d+\left(x_{i}^{2}+y_{i}^{2}\right)(s C d)^{-1}\right)^{-1} \\
& Y_{12}=Y_{21}=\frac{-\left(x_{i}^{2}+y_{i}^{2}\right)}{4 x_{i}}(s L d)^{-1}-\frac{1}{4 x_{i}} s C d+x_{i}\left(s L d+\left(x_{i}^{2}+y_{i}^{2}\right)(s C d)^{-1}\right)^{-1}
\end{aligned}
$$

for the complex pole-zero pairs and

$$
\begin{aligned}
& Y_{11}=Y_{22}=\frac{\alpha_{0}}{2}(s L d)^{-1}+\frac{1}{2 \alpha_{0}} s C d \\
& Y_{12}=Y_{21}=-\frac{\alpha_{0}}{2}(s L d)^{-1}+\frac{1}{2 \alpha_{0}} s C d
\end{aligned}
$$

for the real pole-zero pair. Circuit equivalent models that represents the Y-parameters of (4.20) and (4.21) are shown in Figure 4.4 for a two-conductor lossless transmission line. Figure $4.4 \mathrm{a}$ shows the topology corresponding to the complex pole-zero subsection of (4.20) and Figure 4.4b shows the topology for the real pole-zero subsection of (4.21). MTLs can also be realized in terms of circuit elements. An example of a three-conductor lossless transmission line is shown in Figure 4.5. The MNA matrices of each subsection $G_{i}$ and $C_{i}$ of (4.15) are 


$$
G_{i}=\left[\begin{array}{ccccc}
0 & 0 & 0 & U & U \\
0 & 0 & 0 & U & 0 \\
0 & 0 & 0 & 0 & -U \\
-U & U & 0 & 0 & 0 \\
-U & 0 & U & 0 & 0
\end{array}\right] \quad C_{i}=\left[\begin{array}{cccccc}
\frac{-d}{4 x_{i}} C & 0 & \frac{-d}{4 x_{i}} C & 0 & 0 \\
0 & \frac{x_{i} d}{x_{i}^{2}+y_{i}^{2}} C & \frac{x_{i} d}{x_{i}^{2}+y_{i}^{2}} C & 0 & 0 \\
\frac{-d}{4 x_{i}} C & \frac{x_{i} d}{x_{i}^{2}+y_{i}^{2}} C\left(\frac{d}{4 x_{i}}+\frac{x_{i} d}{x_{i}^{2}+y_{i}^{2}}\right) & 0 & 0 \\
0 & 0 & 0 & \frac{d}{x_{i} L} & 0 \\
0 & 0 & 0 & 0 & \frac{4 x_{i} d}{x_{i}^{2}+y_{i}^{2}} L
\end{array}\right]
$$

for the complex pole-zero pairs and

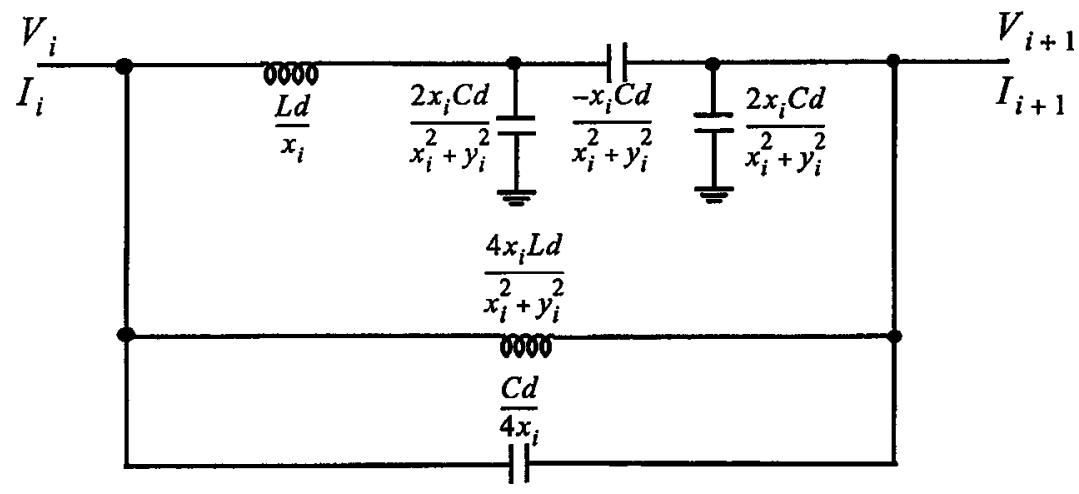

a) Complex pole-zero subsection

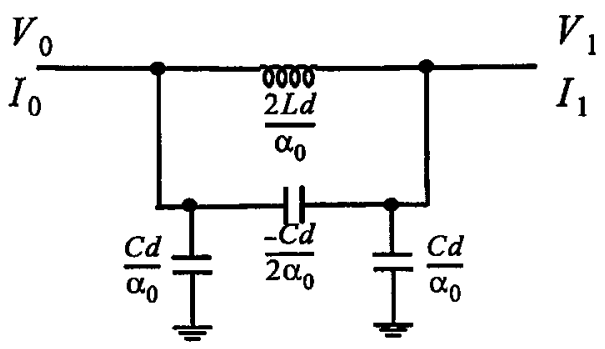

b) Real pole-zero subsection

Figure 4.4 Realization of two-conductor lossless transmission line 


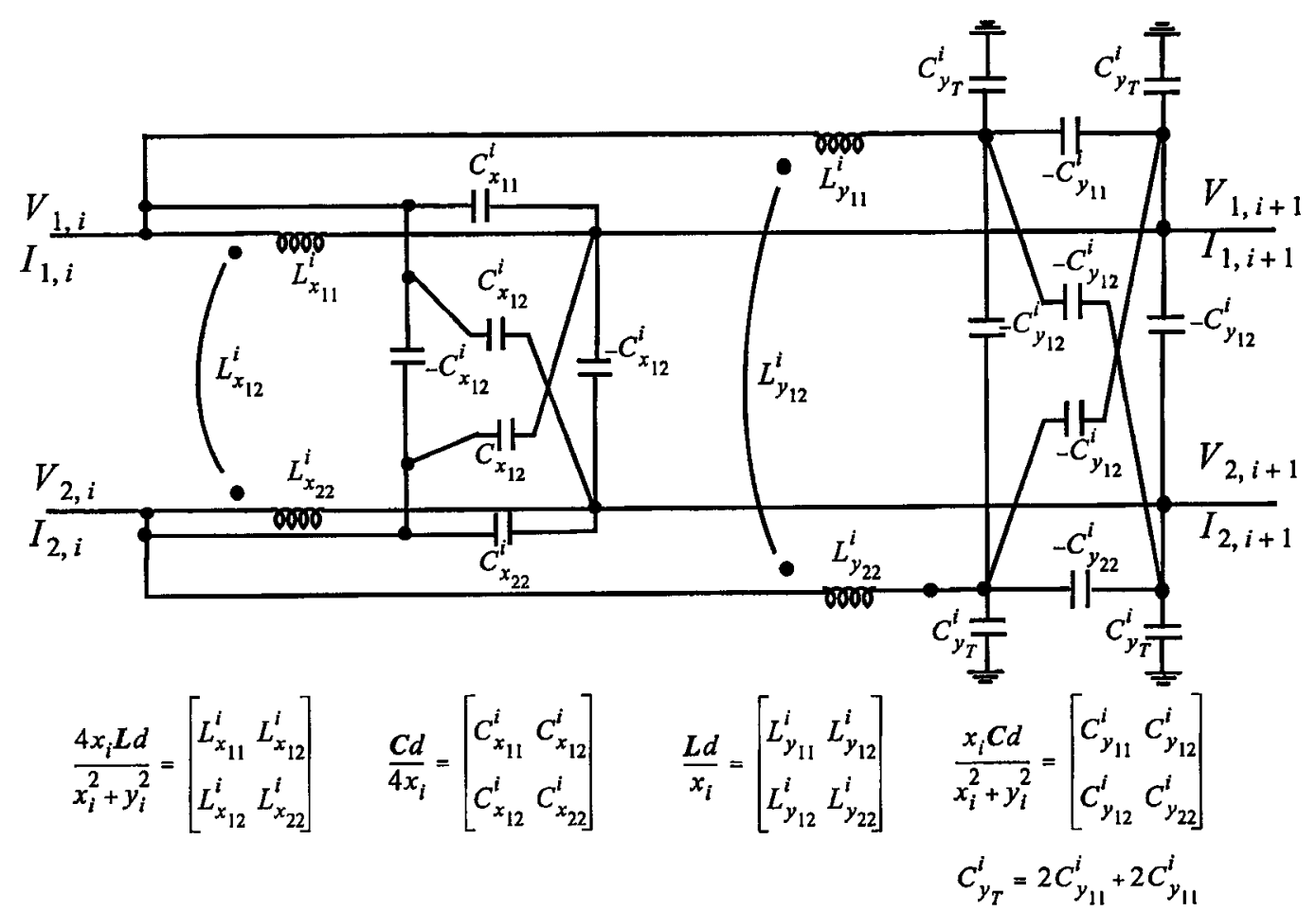

a) Complex pole-zero subsection

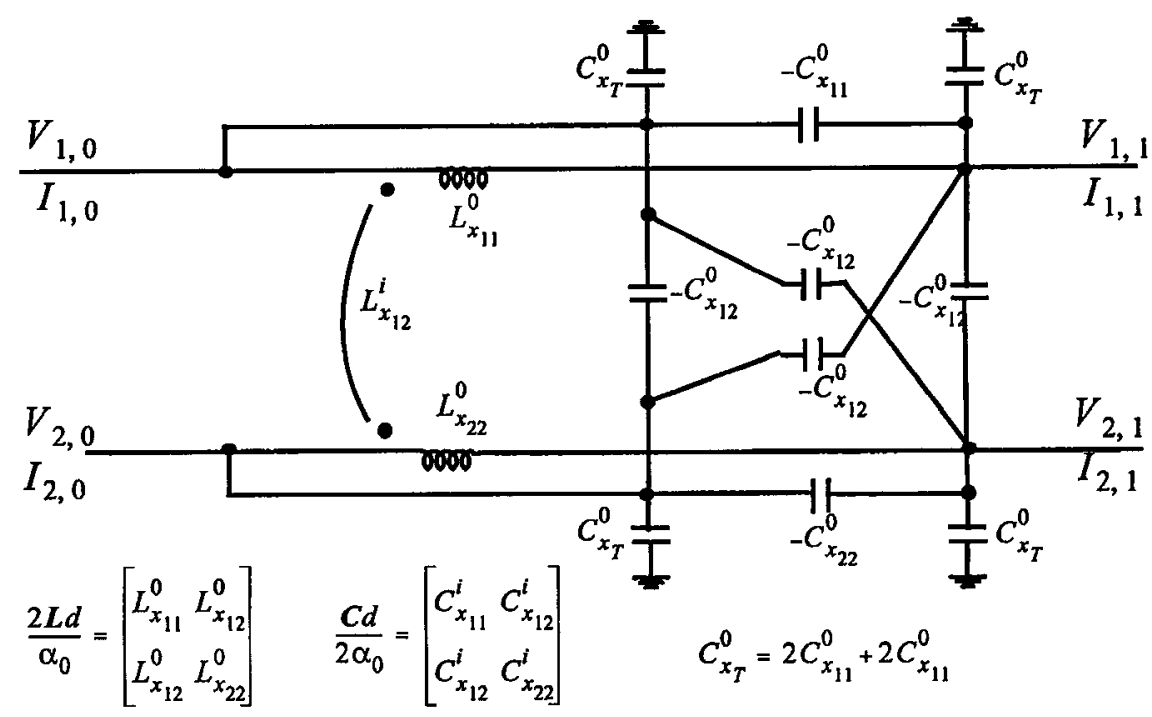

b) Real pole-zero subsection

Figure 4.5 Realization of lossless MTL 


$$
G_{0}=\left[\begin{array}{ccc}
0 & 0 & U \\
0 & 0 & -U \\
-U & U & 0
\end{array}\right] \quad C_{0}=\left[\begin{array}{ccc}
\frac{d}{2 \alpha_{0}} C \frac{d}{2 \alpha_{0}} C & 0 \\
\frac{d}{2 \alpha_{0}} C & \frac{d}{2 \alpha_{0}} C & 0 \\
0 & 0 & \frac{2 d}{\alpha_{0}} L
\end{array}\right]
$$

for the real pole-zero pairs. Equations (4.22) and (4.23) are equivalent to Figure 4.4 and Figure 4.5 and correspond to the MNA stamp of each subsection for lossless transmission lines.

\subsubsection{Lossy Transmission Lines}

For the case of lossy (RLGC) transmission lines, the Y-parameters of (4.13) and (4.14) are

$$
\begin{aligned}
& \boldsymbol{Y}_{11}=\boldsymbol{Y}_{22}=\frac{\left(x_{i}^{2}+y_{i}^{2}\right)}{4 x_{i} d}(\boldsymbol{R}+s L)^{-1}+\frac{d}{4 x_{i}}(\boldsymbol{G}+s \boldsymbol{C})+x_{i}\left((\boldsymbol{R}+s L) d+\frac{\left(x_{i}^{2}+y_{i}^{2}\right)}{d}(\boldsymbol{G}+s \boldsymbol{C})^{-1}\right)^{-1} \\
& \boldsymbol{Y}_{12}=\boldsymbol{Y}_{21}=\frac{-\left(x_{i}^{2}+y_{i}^{2}\right)}{4 x_{i} d}(\boldsymbol{R}+s L)^{-1}-\frac{d}{4 x_{i}}(\boldsymbol{G}+s C)+x_{i}\left((\boldsymbol{R}+s L) d+\frac{\left(x_{i}^{2}+y_{i}^{2}\right)}{d}(\boldsymbol{G}+s C)^{-1}\right)^{-1}
\end{aligned}
$$

for the complex pole-zero pairs and

$$
\begin{aligned}
& \boldsymbol{Y}_{11}=\boldsymbol{Y}_{22}=\frac{\alpha_{0}}{2 d}(\boldsymbol{R}+s \boldsymbol{L})^{-1}+\frac{d}{2 \alpha_{0}}(\boldsymbol{G}+s \boldsymbol{C}) \\
& \boldsymbol{Y}_{12}=\boldsymbol{Y}_{21}=-\frac{\alpha_{0}}{2 d}(\boldsymbol{R}+s \boldsymbol{L})^{-1}+\frac{1}{2 \alpha_{0}}(\boldsymbol{G}+s \boldsymbol{C})
\end{aligned}
$$

for the real pole-zero pair. Circuit equivalent models that represents the Y-parameters of (4.24) and (4.25) are shown in Figure 4.6 for a two-conductor lossy transmission line. Figure 4.6a shows the topology corresponding to the complex pole-zero subsection of (4.24) and Figure 4.6b shows the topology for the real pole-zero subsection of (4.25). MTLs can also be realized in terms of circuit elements. An example of a three-conductor lossy transmission line is shown in Figure 4.7 and Figure 4.8. The MNA matrices of each subsection 
$G_{i}$ and $C_{i}$ of (4.15) are

$$
\begin{aligned}
& \boldsymbol{G}_{i}=\left[\begin{array}{ccccccc}
\left(\frac{x_{i}}{d}+\frac{x_{i}^{2}+y_{i}^{2}}{4 x_{i} d}\right) R^{-1}+\frac{d}{4 x_{i}} G & \frac{-x_{i}}{d} R^{-1} & 0 & \frac{-d}{4 x_{i}} G & \frac{-\left(x_{i}^{2}+y_{i}^{2}\right)}{4 x_{i} d} R^{-1} & 0 & 0 \\
\frac{-x_{i}}{d} R^{-1} & \frac{x_{i}}{d} R^{-1} & 0 & 0 & 0 & U & 0 \\
0 & 0 & \frac{x_{i} d}{x_{i}^{2}+y_{i}^{2}} G & \frac{x_{i} d}{x_{i}^{2}+y_{i}^{2}} G & 0 & -U & 0 \\
\frac{-d}{4 x_{i}} G & 0 & \frac{x_{i} d}{x_{i}^{2}+y_{i}^{2}} G\left(\frac{x_{i} d}{x_{i}^{2}+y_{i}^{2}}+\frac{d}{4 x_{i}}\right) G & 0 & 0 & U \\
\frac{-\left(x_{i}^{2}+y_{i}^{2}\right)}{4 x_{i} d} R^{-1} & 0 & 0 & 0 & \frac{x_{i}^{2}+y_{i}^{2}}{4 x_{i} d} R^{-1} & 0 & -U \\
0 & -U & U & 0 & 0 & 0 & 0 \\
0 & 0 & 0 & -U & U & 0 & 0
\end{array}\right] \\
& C_{i}=\left[\begin{array}{ccccccc}
\frac{d}{4 x_{i}} C & 0 & 0 & \frac{-d}{4 x_{i}} C & 0 & 0 & 0 \\
0 & 0 & 0 & 0 & 0 & 0 & 0 \\
0 & 0 & \frac{x_{i} d}{x_{i}^{2}+y_{i}^{2}} C & \frac{x_{i} d}{x_{i}^{2}+y_{i}^{2}} C & 0 & 0 & 0 \\
\frac{-d}{4 x_{i}} C & 0 & \frac{x_{i} d}{x_{i}^{2}+y_{i}^{2}} C\left(\frac{x_{i} d}{x_{i}^{2}+y_{i}^{2}}+\frac{d}{4 x_{i}}\right) & C & 0 & 0 & 0 \\
0 & 0 & 0 & 0 & 0 & 0 & 0 \\
0 & 0 & 0 & 0 & 0 & \frac{d}{x_{i}} L & 0 \\
0 & 0 & 0 & 0 & 0 & 0 & \frac{4 x_{i} d}{x_{i}^{2}+y_{i}^{2}} L
\end{array}\right]
\end{aligned}
$$

for the complex pole-zero pairs and

$$
G_{0}=\left[\begin{array}{cccc}
\frac{a_{0}}{2 d} R^{-1}+\frac{d}{2 a_{0}} G & \frac{-a_{0}}{2 d} R^{-1} & \frac{d}{2 a_{0}} G & 0 \\
\frac{-a_{0}}{2 d} R^{-1} & \frac{a_{0}}{2 d} R^{-1} & 0 & U \\
\frac{d}{2 a_{0}} G & 0 & \frac{d}{2 a_{0}} G & -U \\
0 & -U & U & 0
\end{array}\right] \quad C_{0}=\left[\begin{array}{cccc}
\frac{d}{2 a_{0}} C & 0 & \frac{d}{2 a_{0}} C & 0 \\
0 & 0 & 0 & 0 \\
\frac{d}{2 a_{0}} C & 0 & \frac{d}{2 a_{0}} C & 0 \\
0 & 0 & 0 & \frac{2 d}{a_{0}} L
\end{array}\right]
$$


for the real pole-zero pairs. Equations (4.26) and (4.27) correspond to the MNA stamp of each subsection for lossy transmission lines.

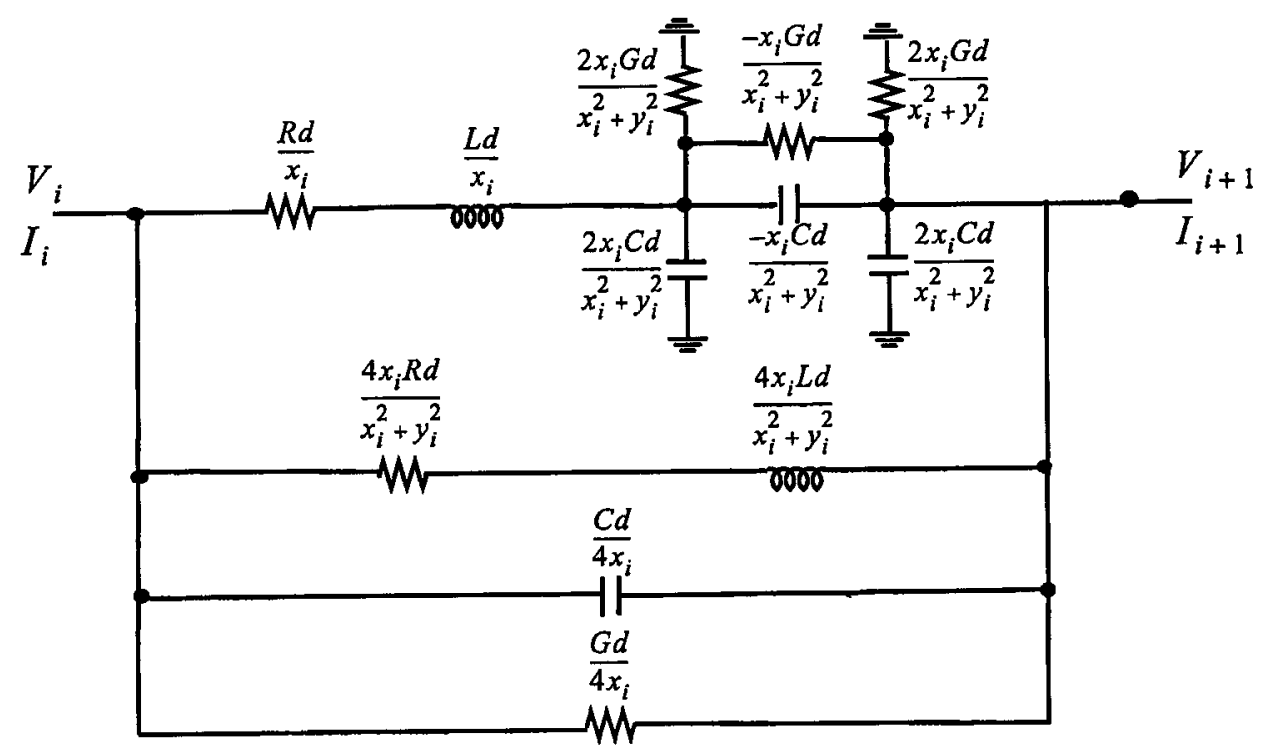

a) Complex pole-zero subsection

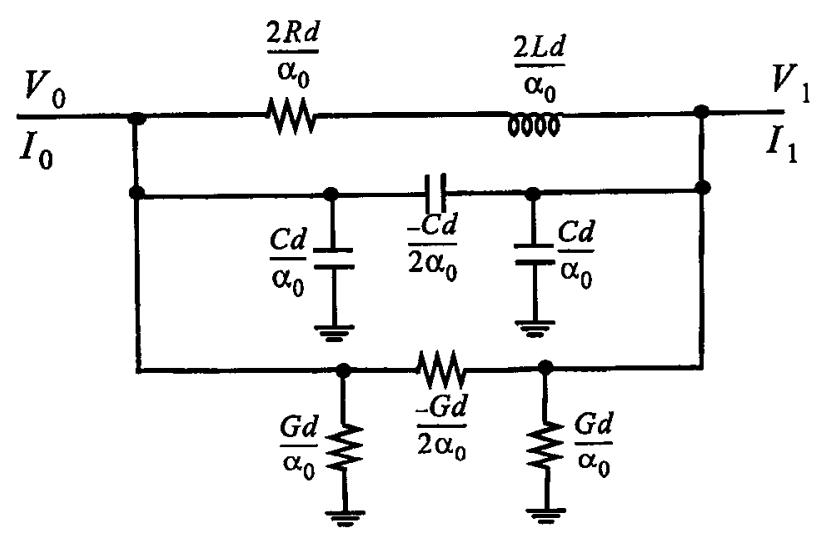

b) Real pole-zero subsection

Figure 4.6 Realization of two-conductor lossy transmission line 


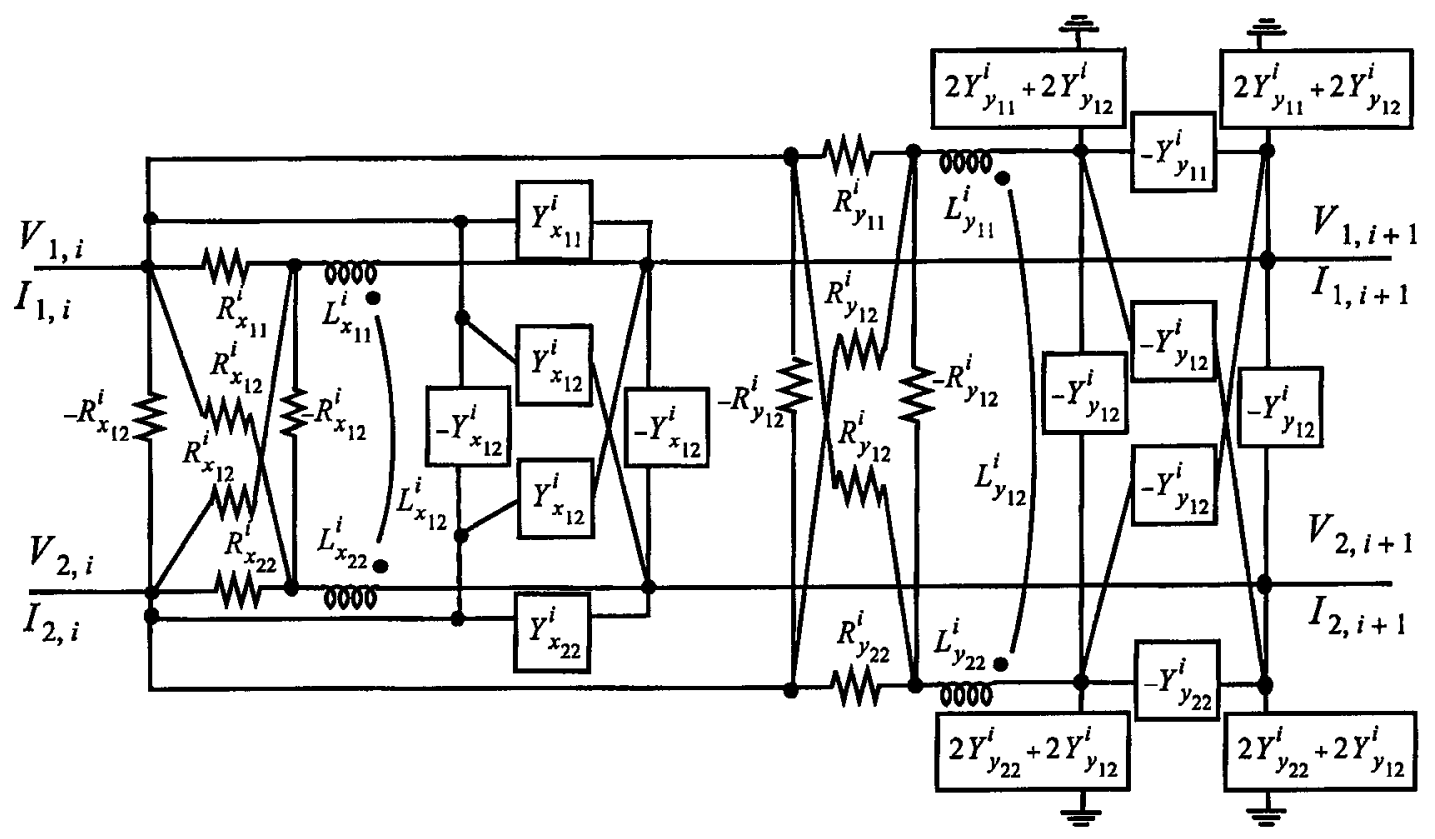

a) Complex pole-zero subsection

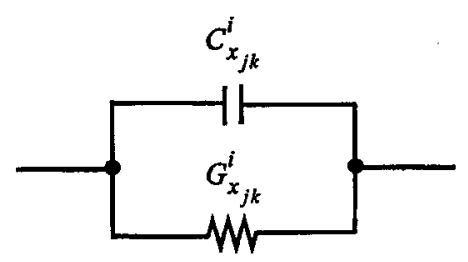

b) $Y_{x_{j k}}^{i}$ circuit

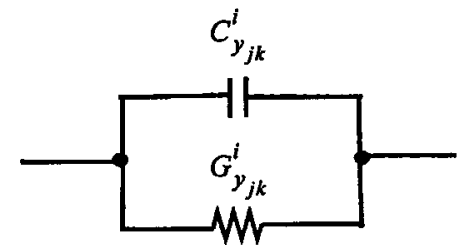

c) $Y_{y_{j k}}^{i}$ circuit

$$
\begin{array}{ccc}
\left(\frac{4 x_{i} R d}{x_{i}^{2}+y_{i}^{2}}\right)^{-1}=\left[\begin{array}{cc}
\frac{1}{R_{x_{11}}^{i}} & \frac{1}{R_{x_{12}}^{i}} \\
\frac{1}{R_{x_{12}}^{i}} & \frac{1}{R_{x_{22}}^{i}}
\end{array}\right] & \frac{C d}{4 x_{i}}=\left[\begin{array}{ll}
C_{x_{11}}^{i} & C_{x_{12}}^{i} \\
C_{x_{12}}^{i} & C_{x_{22}}^{i}
\end{array}\right] & \left(\frac{R d}{x_{i}}\right)^{-1}=\left[\begin{array}{cc}
\frac{1}{R_{y_{11}}^{i}} \frac{1}{R_{y_{12}}^{i}} \\
\frac{1}{R_{y_{12}}^{i}} \frac{1}{R_{y_{22}}^{i}}
\end{array}\right] \quad \frac{x_{i} C d}{x_{i}^{2}+y_{i}^{2}}=\left[\begin{array}{ll}
C_{y_{11}}^{i} & C_{y_{12}}^{i} \\
C_{y_{12}}^{i} & C_{y_{22}}^{i}
\end{array}\right] \\
\frac{4 x_{i} L d}{x_{i}^{2}+y_{i}^{2}}=\left[\begin{array}{ll}
L_{x_{11}}^{i} & L_{x_{12}}^{i} \\
L_{x_{12}}^{i} & L_{x_{22}}^{i}
\end{array}\right] \quad \frac{G d}{4 x_{i}}=\left[\begin{array}{ll}
G_{x_{11}}^{i} & G_{x_{12}}^{i} \\
G_{x_{12}}^{i} & G_{x_{22}}^{i}
\end{array}\right] & \frac{L d}{x_{i}}=\left[\begin{array}{ll}
L_{y_{11}}^{i} & L_{y_{12}}^{i} \\
L_{y_{12}}^{i} & L_{y_{22}}^{i}
\end{array}\right] \quad \frac{x_{i} G d}{x_{i}^{2}+y_{i}^{2}}=\left[\begin{array}{ll}
G_{y_{11}}^{i} & G_{y_{12}}^{i} \\
G_{y_{12}}^{i} & G_{y_{22}}^{i}
\end{array}\right]
\end{array}
$$

Figure 4.7 Realization of lossy MTL for complex pole-zero subsection of (4.24) 


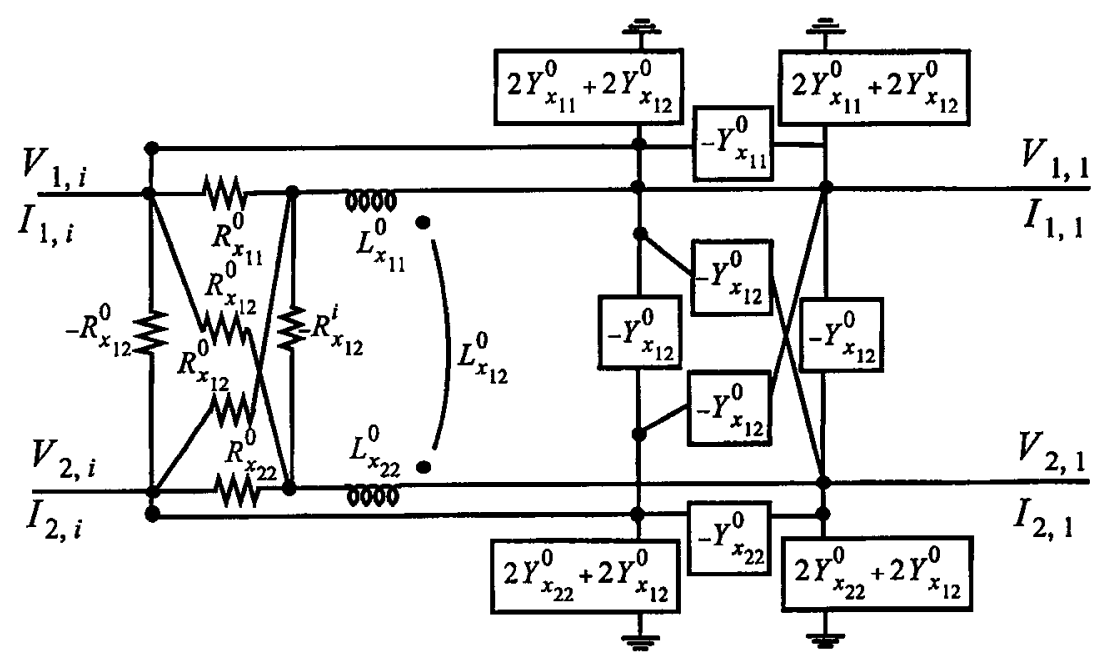

a) Real pole-zero subsection

$$
\begin{gathered}
\text { b) } Y_{x_{j k}}^{0} \text { circuit } \\
\left(\frac{2 R d}{\alpha_{0}}\right)^{-1}=\left[\begin{array}{ll}
\frac{1}{R_{x_{11}}^{0}} \frac{1}{R_{x_{12}}^{0}} \\
\frac{1}{R_{x_{12}}^{0}} \frac{1}{R_{x_{22}}^{0}}
\end{array}\right] \quad \frac{2 L d}{\alpha_{0}}=\left[\begin{array}{ll}
L_{x_{11}}^{0} & L_{x_{12}}^{0} \\
L_{x_{12}}^{0} & L_{x_{22}}^{0}
\end{array}\right] \quad \frac{C d}{2 \alpha_{0}}=\left[\begin{array}{ll}
C_{x_{11}}^{i} & C_{x_{12}}^{i} \\
C_{x_{12}}^{i} & C_{x_{22}}^{i}
\end{array}\right] \quad \frac{G d}{2 \alpha_{0}}=\left[\begin{array}{ll}
G_{x_{11}}^{i} & G_{x_{12}}^{i} \\
G_{x_{12}}^{i} & G_{x_{22}}^{i}
\end{array}\right]
\end{gathered}
$$

Figure 4.8 Realization of lossy MTL for real pole-zero subsection of (4.25)

\subsubsection{Example of MRA Circuit Formulation}

To illustrate the MNA formulation of the MRA algorithm an example is provided. Consider the RC transmission line network of Figure 4.9a. The corresponding MRA macromodel for $\operatorname{order} N=1$ is shown in Figure 4.9b. The MNA equations of Figure $4.9 \mathrm{~b}$ can be written in the form of (4.2), as

$$
C_{\pi} \frac{d}{d t} x_{\pi}(t)+G_{\pi} x_{\pi}(t)=b_{\pi}(t)
$$


where $G_{\pi}=G_{a}+G_{T}$ and $C_{\pi}=C_{a}+C_{T}$. The matrices $G_{a}$ and $C_{a}$ correspond to the MNA equations of the lumped circuit elements, as

$$
\boldsymbol{G}_{\boldsymbol{a}}=\left[\begin{array}{cccc}
0 & 1 & 0 & 0 \\
1 & G_{1} & -G_{1} & 0 \\
0 & -G_{1} & G_{1} & 0 \\
0 & 0 & 0 & 0
\end{array}\right] \quad \boldsymbol{C}_{a}=\left[\begin{array}{cccc}
0 & 0 & 0 & 0 \\
0 & 0 & 0 & 0 \\
0 & 0 & 0 & 0 \\
0 & 0 & 0 & C_{1}
\end{array}\right] \quad \boldsymbol{x}_{\pi}(t)=\left[\begin{array}{c}
I_{V} \\
V_{1} \\
V_{2} \\
V_{3}
\end{array}\right] \quad \boldsymbol{b}_{\pi}(t)=\left[\begin{array}{l}
V \\
0 \\
0 \\
0
\end{array}\right]
$$

The matrices $G_{T}$ and $C_{T}$ correspond to the stamp of the MRA macromodel as described by (4.15). For the example shown in Figure 4.9, the matrices become $G_{T}=\Psi_{0}{ }^{T} G_{0} \psi_{0}$ and $C_{r}=\psi_{0}{ }^{T} C_{0} \psi_{0}$, where

$$
\boldsymbol{G}_{0}=\left[\begin{array}{ll}
\frac{\alpha_{0}}{2 R d} & \frac{-\alpha_{0}}{2 R d} \\
\frac{-\alpha_{0}}{2 R d} & \frac{\alpha_{0}}{2 R d}
\end{array}\right] \quad C_{0}=\left[\begin{array}{ll}
\frac{C d}{2 \alpha_{0}} & \frac{C d}{2 \alpha_{0}} \\
\frac{C d}{2 \alpha_{0}} & \frac{C d}{2 \alpha_{0}}
\end{array}\right] \quad \Psi_{0}=\left[\begin{array}{llll}
0 & 0 & 1 & 0 \\
0 & 0 & 0 & 1
\end{array}\right]
$$

The matrices $G_{0}$ and $C_{0}$ correspond to the RC formulation of the real pole-zero subsection of (4.19) and $\psi_{0}$ is a selector matrix that maps $G_{0}$ and $C_{0}$ to the rest of the network equations. It should be noted that for the RC, lossless and lossy (RLGC) transmission line models, the formulation of the MRA macromodel is obtained analytically in term of predetermined coefficients (i.e. $\alpha_{0}, x_{i}$ and $y_{i}$ ) and the p.u.l. parameters of the transmission line.

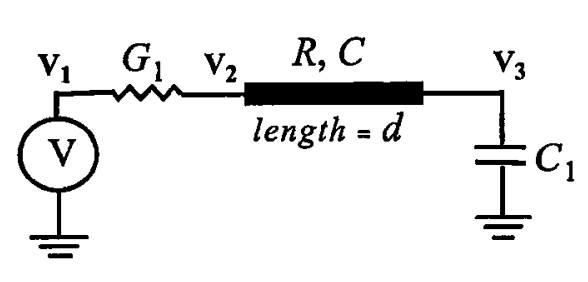

a) $\mathrm{RC}$ transmission line network

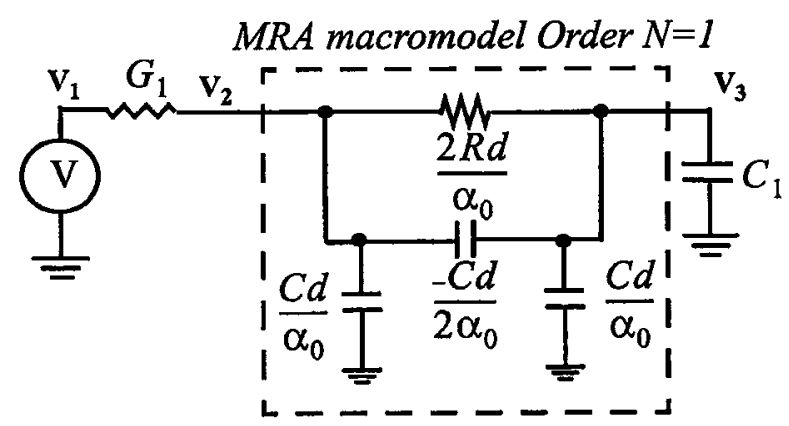

b) MRA macromodel (order $N=1$ )

Figure 4.9 Example circuit formulation of MRA macromodel 


\subsection{Application of MRA to Incident Field Coupling}

This section briefly outlines the modeling of incident field coupling to lossy MTLs using the MRA macromodel [64]-[65], [89].

\subsubsection{Formulation of Incident Field Forcing Function}

Consider the solution of a $m+1$-conductor coupled transmission line described by telegrapher's equation in the Laplace domain

$$
\left[\begin{array}{r}
V(s, d) \\
-I(s, d)
\end{array}\right]=e^{\hat{Z}_{d}}\left[\begin{array}{c}
\boldsymbol{V}(s, 0) \\
\boldsymbol{I}(s, 0)
\end{array}\right]+\boldsymbol{B}(s)
$$

where

$$
\hat{Z}=\left[\begin{array}{cc}
0 & -(R+s L) \\
-(G+s C) & 0
\end{array}\right]
$$

The forcing function $\boldsymbol{B}(s)$ is due to incident fields. In the case of uniform plane wave excitation of traces, it is possible to express the incoming field analytically (see Figure 4.10).

$$
\vec{E}(x, y, z)=\left(A_{x} \hat{a}_{x}+A_{y} \hat{a}_{y}+A_{z} \hat{a}_{z}\right) e^{-s \kappa_{x} x} e^{-s \kappa_{y} y} e^{-s \kappa_{z} z}
$$

where $A_{\mathrm{x}}, A_{\mathrm{y}}, A_{\mathrm{z}}$ are the direction cosines of the incoming wave [2] and

$$
\kappa_{z}=-\frac{\sin \theta \sin \phi}{c} ; \quad \kappa_{x}=-\frac{\cos \theta}{c} ; \quad \kappa_{y}=-\frac{\sin \theta \cos \phi}{c}
$$

with $c$ being the speed of phase front. Assuming that the reference conductor is positioned at the origin, the forcing function $\boldsymbol{B}(s)$ can be written as [2], [89],

$$
\boldsymbol{B}(s)=e^{\hat{Z} d} \int_{0}^{d} e^{-\hat{Z} z} \Gamma(s) e^{-s \kappa_{z} z} d z
$$

The term $\Gamma(s)$ in (4.35) describes the interaction of the incoming fields with the lines in the transverse plane as 


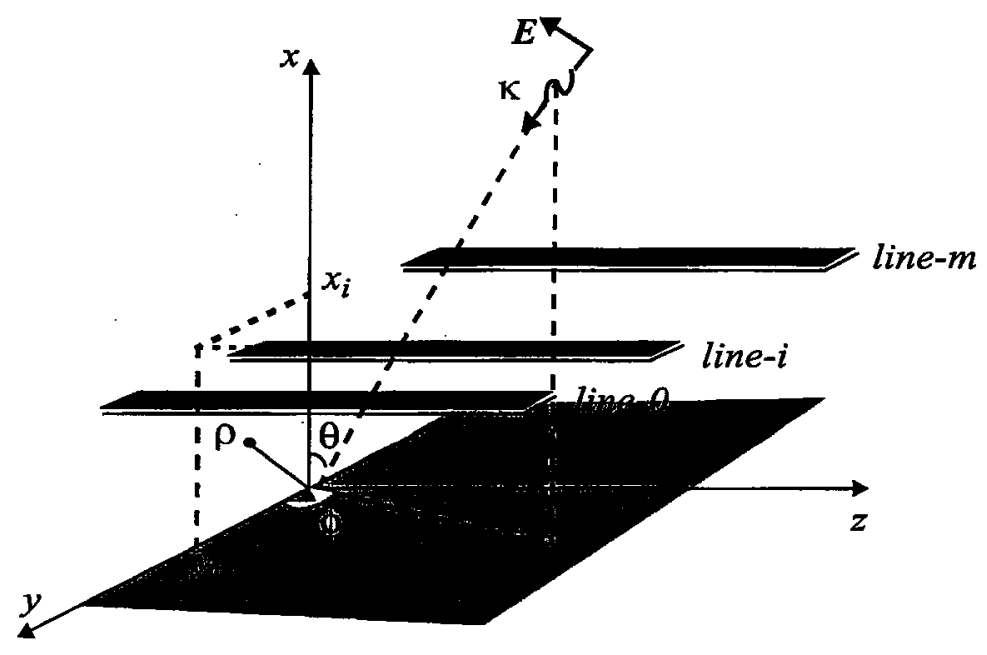

Figure 4.10 Definition of parameters for incident field coupling

$$
\Gamma(s)=\left[\begin{array}{c}
\tilde{\boldsymbol{V}}_{F}(s) \\
\tilde{\boldsymbol{I}}_{F}(s)
\end{array}\right]
$$

For a transmission medium with no solid ground plane, the terms on the right hand side of (4.36) can be expressed as

$$
\begin{gathered}
\tilde{\boldsymbol{V}}_{F}(s)=\left[\begin{array}{c}
\left(-\frac{\kappa_{z} \Phi_{i}^{+}}{\psi_{i}^{+}}+A_{z}\right)\left(e^{-s \psi_{i}^{+}}-1\right) \\
\vdots
\end{array}\right](d-z) \\
\tilde{\boldsymbol{I}}_{F}(s)=\left(\frac{G}{s}+C\right)\left[\begin{array}{c}
\vdots \\
\frac{\Phi_{i}^{+}}{\psi_{i}^{+}}\left(e^{-s \psi_{i}^{+}}-1\right) \\
\vdots
\end{array}\right]
\end{gathered}
$$

and for a microstrip structure, which is backed by a ground plane (4.36) is expressed as 


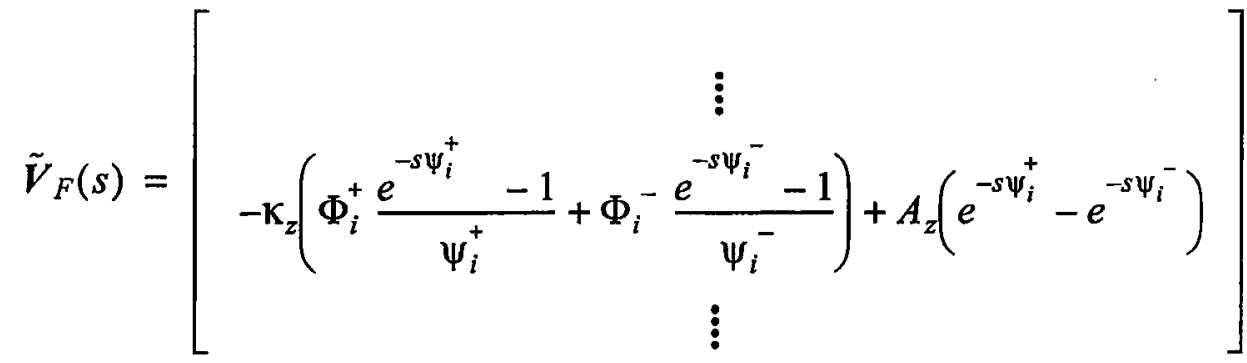

$$
\begin{aligned}
& \tilde{I}_{F}(s)=\left(\frac{G}{s}+C\right)\left[\begin{array}{c}
\vdots \\
\Phi_{i}^{+} \frac{e^{-s \psi_{i}^{+}}-1}{\psi_{i}^{+}}+\Phi_{i}^{-} \frac{e^{-s \psi_{i}^{-}}-1}{\psi_{i}^{-}} \\
\vdots
\end{array}\right]
\end{aligned}
$$

In (4.37)-(4.38), the subscript $i$ refers to the $i$ th conductor and $\Phi_{\mathrm{i}}, \psi_{\mathrm{i}}$ are defined as [2],

$$
\Phi_{i}^{ \pm}=A_{x} x_{i} \pm A_{y} y_{i} ; \quad \psi_{i}^{ \pm}=\kappa_{x} x_{i} \pm \kappa_{y} y_{i}
$$

Next, define

$$
\boldsymbol{H}=-\hat{\boldsymbol{Z}}-\boldsymbol{s} \boldsymbol{\kappa}_{z} \boldsymbol{U}
$$

where $U$ is the identity matrix. The solution to (4.35) results in

$$
\boldsymbol{B}(s)=e^{\hat{\boldsymbol{Z}} d}\left(e^{\boldsymbol{H} d}-\boldsymbol{U}\right) \boldsymbol{H}^{-1} \Gamma(s)
$$

which can be further simplified as

$$
\boldsymbol{B}(s)=\left(e^{-s \kappa_{z} U d}-e^{\hat{Z} d}\right) H^{-1} \Gamma(s)
$$

Incorporating (4.42) with (4.31), yields

$$
\left[\begin{array}{c}
V(s, d) \\
-I(s, d)
\end{array}\right]=e^{2 d}\left[\begin{array}{c}
\boldsymbol{V}(s, 0) \\
I(s, 0)
\end{array}\right]+\left(e^{-s \kappa_{2} U d}-e^{Z d}\right) H^{-1} \Gamma(s)
$$

The difficulty in obtaining a macromodel for (4.42)-(4.43) stems from the fact that $e^{Z d}$ and $\boldsymbol{B}(s)$ do not have a direct analytic time-domain representation. The space and time dependency of these distributed functions makes a brute force frequency to time-domain conversion impractical. The handling of the term $e^{\hat{Z} d}$ in time-domain simulators has been addressed in section 4.3. However, addressing the term $B(s)$ is still a major concern for 
time-domain simulators. In the following section, the time-domain formulation of $\boldsymbol{B}(s)$ is briefly described. Compared to existing algorithms [38]-[39], a major advantage of the proposed algorithm is that it can account for the losses, and can be extended to include frequency-dependent parameters [65].

\subsubsection{Time-domain Macromodel for Equivalent Sources}

This section describes the time-domain formulation for $\boldsymbol{B}(s)$. Equation (4.31) can be rewritten as

$$
\left[\begin{array}{c}
V(s, d) \\
-I(s, d)
\end{array}\right]=\left[\begin{array}{ll}
T_{11}(s, d) & T_{12}(s, d) \\
T_{21}(s, d) & T_{22}(s, d)
\end{array}\right]\left[\begin{array}{c}
V(s, 0) \\
I(s, 0)
\end{array}\right]+\left[\begin{array}{c}
V_{F T}(s, d) \\
I_{F T}(s, d)
\end{array}\right]
$$

with $\boldsymbol{T}$ being the state-transition matrix. An equivalent representation in terms of the admittance parameters can be written as (for the sake of clarity, the complex frequency term $s$ is omitted)

$$
\begin{aligned}
{\left[\begin{array}{l}
I(0) \\
I(d)
\end{array}\right]=} & {\left[\begin{array}{cc}
-T_{12}(d)^{-1} T_{11}(d) & T_{12}(d)^{-1} \\
-T_{21}(d)+T_{22}(d) T_{12}(d)^{-1} T_{11}(d) & -T_{22}(d) T_{12}(d)^{-1}
\end{array}\right]\left[\begin{array}{l}
V(0) \\
V(d)
\end{array}\right] } \\
& +\left[\begin{array}{cc}
-T_{12}(d)^{-1} & 0 \\
T_{22}(d) T_{12}(d)^{-1} & -U
\end{array}\right]\left[\begin{array}{l}
V_{F T}(d) \\
I_{F T}(d)
\end{array}\right]
\end{aligned}
$$

The second term on the right hand of (4.45) represents the short circuit current sources. These sources can be superimposed at the terminals of the line during transient simulation as shown in Figure 4.11.

To obtain a time-domain macromodel for the short circuit current sources, $I_{s c}(0)$ and $I_{s c}(d)$ are expressed in terms of rational functions in $s$ and delay elements, as follows. Using (4.42), (4.45), short circuit current sources can be written as

$$
\left[\begin{array}{c}
\boldsymbol{I}_{s c}(0) \\
\boldsymbol{I}_{s c}(d)
\end{array}\right]=\left[\begin{array}{cc}
-\boldsymbol{T}_{12}(d)^{-1} & 0 \\
T_{22}(d) T_{12}(d)^{-1} & -U
\end{array}\right]\left(\left(e^{-s \kappa_{z} v d}-\left[\begin{array}{ll}
\boldsymbol{T}_{11}(d) & \boldsymbol{T}_{12}(d) \\
\boldsymbol{T}_{21}(d) & \boldsymbol{T}_{22}(d)
\end{array}\right]\right) H^{-1} \Gamma(s)\right)
$$




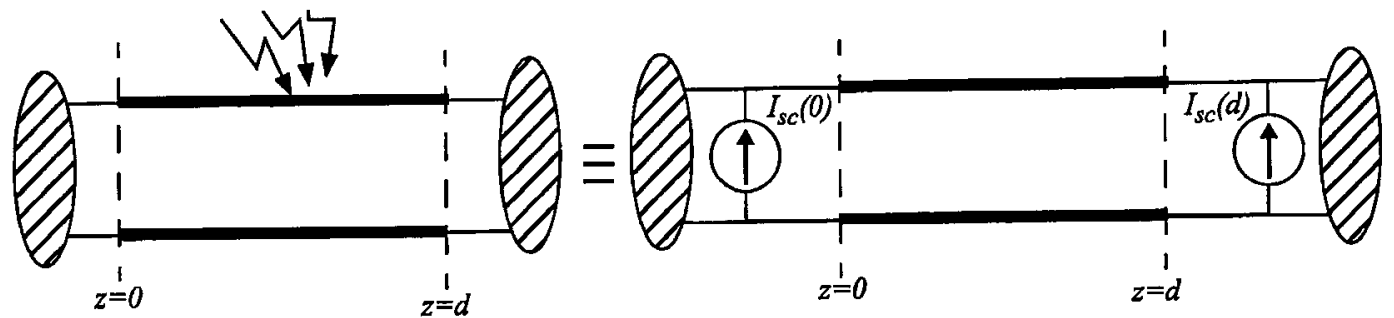

Figure 4.11 Equivalent circuit of incident field coupling to transmission-lines

Eq (4.46), can be simplified to

$$
\left[\begin{array}{l}
I_{s c}(0) \\
I_{s c}(d)
\end{array}\right]=W H^{-1} \Gamma(s)
$$

where

$$
\begin{gathered}
W=\left[\begin{array}{ll}
W_{11}(d) & W_{12}(d) \\
W_{21}(d) & W_{22}(d)
\end{array}\right] \\
W_{11}(d)=-T_{12}(d)^{-1}\left(e^{-s \kappa_{z} U d}-T_{11}(d)\right) \\
W_{12}(d)=U \\
W_{21}(d)=T_{22}(d) T_{12}(d)^{-1}\left(e^{-s \kappa_{z} U d}-T_{11}(d)\right)+T_{21}(d) \\
W_{22}(d)=-e^{-s \kappa_{z} U}
\end{gathered}
$$

The term $\Gamma(s)$ in (4.36)-(4.39) and (4.46)-(4.47) contributes to current sources with a single pole at the origin along with time delay functions composed of incident field angular parameters and line positions in the transverse plane.

Next, the term $H^{-1}$ is converted to a rational-function form (in terms of poles and residues) as follows. From (4.40), $\boldsymbol{H}^{-1}$ can be written as

$$
\boldsymbol{H}^{-1}=(\boldsymbol{D}+s \boldsymbol{E})^{-1}
$$

$$
D=\left[\begin{array}{ll}
0 & R \\
G & 0
\end{array}\right] \quad E=\left[\begin{array}{cc}
-\kappa_{z} U & L \\
C & -\kappa_{z} U
\end{array}\right]
$$

Equation (4.50) can be re-written as 


$$
\boldsymbol{H}^{-1}=\left(\boldsymbol{U}+s D^{-1} E\right)^{-1} \boldsymbol{D}^{-1}
$$

Next, the matrix $D^{-1} E$ is expressed in terms of its eigenvalues and eigenvectors using a similarity transformation as

$$
D^{-1} E=F \Lambda F^{-1}
$$

where $\Lambda=\operatorname{diag}\left(\lambda_{1}, \lambda_{2}, \ldots\right)$ is a diagonal matrix containing the eigenvalues of $D^{-1} E$ and $\boldsymbol{F}$ contains the eigenvectors of $D^{-1} E$.

Next, substituting (4.53) into (4.52), yields

$$
H^{-1}=F(U+s \Lambda)^{-1} F^{-1} D^{-1}
$$

The poles of $\boldsymbol{H}^{-1}$ are the negative reciprocal of the eigenvalues $\lambda_{i}$ and the corresponding residues of the poles are obtained in terms of the entries of $\boldsymbol{D}$ and $\boldsymbol{F}$.

Similarly, the matrix $W$ of (4.47) can be expressed in a rational form using the MRA algorithm to represent the state-transition matrix $e^{Z d}$. For example, replacing $T$ in (4.49) by its equivalent MRA approximation, $W_{11}(d)$ can be expressed as [64],

$$
\begin{aligned}
W_{11}(d) & =\boldsymbol{T}_{12}(d)^{-1} \boldsymbol{T}_{11}(d)-\boldsymbol{T}_{12}(d)^{-1} e^{-s K_{z} U} \\
& =\left(K_{1} s+K_{0}+\sum_{i=0} \frac{\boldsymbol{r}_{\mathbf{1}_{i}}}{s-p_{i}}\right)+\left(\boldsymbol{K}_{3} s+K_{2}+\sum_{i=0} \frac{\boldsymbol{r}_{\mathbf{2}_{i}}}{s-p_{i}}\right) e^{-s K_{z} U d}
\end{aligned}
$$

where $p_{i}$ represent the poles of the rational function, $\boldsymbol{r}_{\mathbf{1}_{i}}, \boldsymbol{r}_{\mathbf{2}_{i}}$ are the corresponding residue matrices of dimensions $m \times m$ and $K_{0}, K_{1}, K_{2}, K_{3}$ are constant matrices of dimensions $m \times m$.

Using (4.54)-(4.55), the short-circuit-current sources in (4.47) can be described as rational-functions in $s$ and time-delay elements, which can be easily converted to equivalent time-domain sources (suitable for SPICE simulation). 


\subsection{Numerical Examples}

Four examples are presented in this section to demonstrate the validity of the proposed approach. The simulations results are compared with the conventional lumped segmentation model [2] and/or 'Exact' analysis of the distributed transmission line equations. Within the context of this section, 'Exact' analysis refers to solving the equations of the linear subnetwork analytically through eigenvalue/eigenvector analysis. The simulations are obtained using HSPICE [93] and circuit equivalent realization of macromodels, on a SUN Blade-1000 workstation with $900 \mathrm{MHz}$ UltraSPARC-III CPU.

\subsubsection{Example 4.1}

A coupled RC distributed network is shown in Figure 4.12. The p.u.l. parameters of the line are

$$
\begin{gathered}
C=\left[\begin{array}{cccccccc}
0.9350 & -0.2941 & -0.04880 & -0.02222 & -0.01437 & -0.01107 & -0.01018 & -0.01658 \\
-0.2941 & 1.0650 & -0.2726 & -0.03899 & -0.01588 & -0.00966 & -0.00749 & -0.01017 \\
-0.04880 & -0.2726 & 1.0680 & -0.2710 & -0.03804 & -0.01534 & -0.00966 & -0.01106 \\
-0.02222 & -0.03899 & -0.2710 & 1.0690 & -0.2707 & -0.03805 & -0.01587 & -0.01435 \\
-0.01437 & -0.01588 & -0.03804 & -0.2707 & 1.0690 & -0.2710 & -0.03899 & -0.02220 \\
-0.01107 & -0.00966 & -0.01534 & -0.03805 & -0.2710 & 1.0680 & -0.2726 & -0.04880 \\
-0.01018 & -0.00749 & -0.00966 & -0.01587 & -0.03899 & -0.2726 & 1.0650 & -0.2941 \\
-0.01658 & -0.01017 & -0.01106 & -0.01435 & -0.02220 & -0.04880 & -0.2941 & 0.9350
\end{array}\right] p F / \mathrm{cm} \\
R= \\
{\left[\begin{array}{cccccccc}
250 & 0 & 0 & 0 & 0 & 0 & 0 & 0 \\
0 & 250 & 0 & 0 & 0 & 0 & 0 & 0 \\
0 & 0 & 250 & 0 & 0 & 0 & 0 & 0 \\
0 & 0 & 0 & 250 & 0 & 0 & 0 & 0 \\
0 & 0 & 0 & 0 & 250 & 0 & 0 & 0 \\
0 & 0 & 0 & 0 & 0 & 250 & 0 & 0 \\
0 & 0 & 0 & 0 & 0 & 0 & 250 & 0 \\
0 & 0 & 0 & 0 & 0 & 0 & 0 & 250
\end{array}\right] \Omega / \mathrm{cm}}
\end{gathered}
$$

and the length of the line is $0.5 \mathrm{~cm}$. The off diagonal p.u.1. resistances are zero since the return line is assumed to be lossless. At the near end, transistor resistances are modeled as $81 \Omega$ and at the far end transistor load capacitances are modeled as $0.15 \mathrm{pF}$. The accuracy of the proposed algorithm is compared with 200 conventional lumped sections (which is sufficiently enough to obtain an accurate simulation). The transient responses correspond- 
ing to a unit step with rise time of 0.1ns are shown in Figure 4.13. It can be noticed, that the transient response using the proposed algorithm with order 2 matches accurately with the conventional 200 lumped segment approximation.

The efficiency and accuracy of the proposed method is shown in Table 4.1 in terms of number of sections, number of poles, $\mathrm{CPU}$ time and maximum error. The maximum error is the maximum difference in the transient response between the proposed method and the 200 conventional lumped segmentation model. The results show that the conventionallumped approximation with 640 poles is less accurate than a 8 pole approximation using the proposed algorithm.

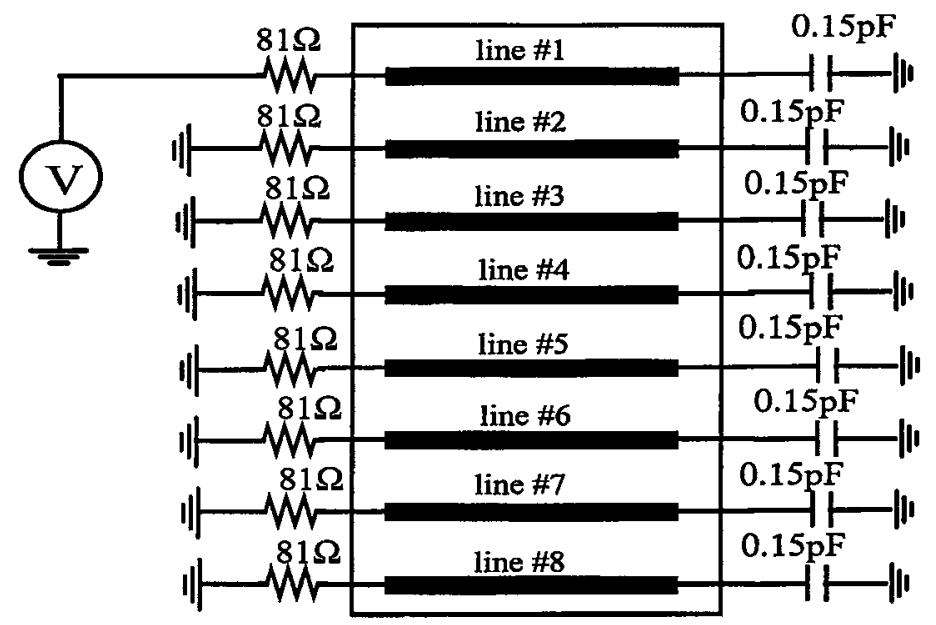

Figure 4.12 Coupled RC distributed network (Example 4.1)

\begin{tabular}{c|c|c|c|c|c|c|c}
\hline \multicolumn{4}{c|}{ Proposed } & \multicolumn{4}{c}{ Conventional Lumped Segmentation } \\
\hline Order & $\begin{array}{c}\text { \# of } \\
\text { poles }\end{array}$ & $\begin{array}{c}\text { CPU time } \\
\text { (seconds) }\end{array}$ & $\begin{array}{c}\mid \text { Maximum } \\
\text { Error } \mid\end{array}$ & Sections & $\begin{array}{c}\text { \# of } \\
\text { poles }\end{array}$ & $\begin{array}{c}\text { CPU time } \\
\text { (seconds) }\end{array}$ & $\begin{array}{c}\mid \text { Maximum } \\
\text { Error }\end{array}$ \\
\hline 2 & 8 & 0.15 & $5.601 \mathrm{e}-4$ & 21 & 160 & 0.41 & $4.732 \mathrm{e}-3$ \\
\hline 3 & 16 & 0.20 & $5.800 \mathrm{e}-5$ & 41 & 320 & 0.78 & $2.160 \mathrm{e}-3$ \\
\hline 4 & 24 & 0.25 & $5.990 \mathrm{e}-6$ & 81 & 640 & 1.54 & $8.194 \mathrm{e}-4$ \\
\hline
\end{tabular}

Table 4.1 Accuracy vs. Efficiency (Example 4.1) 


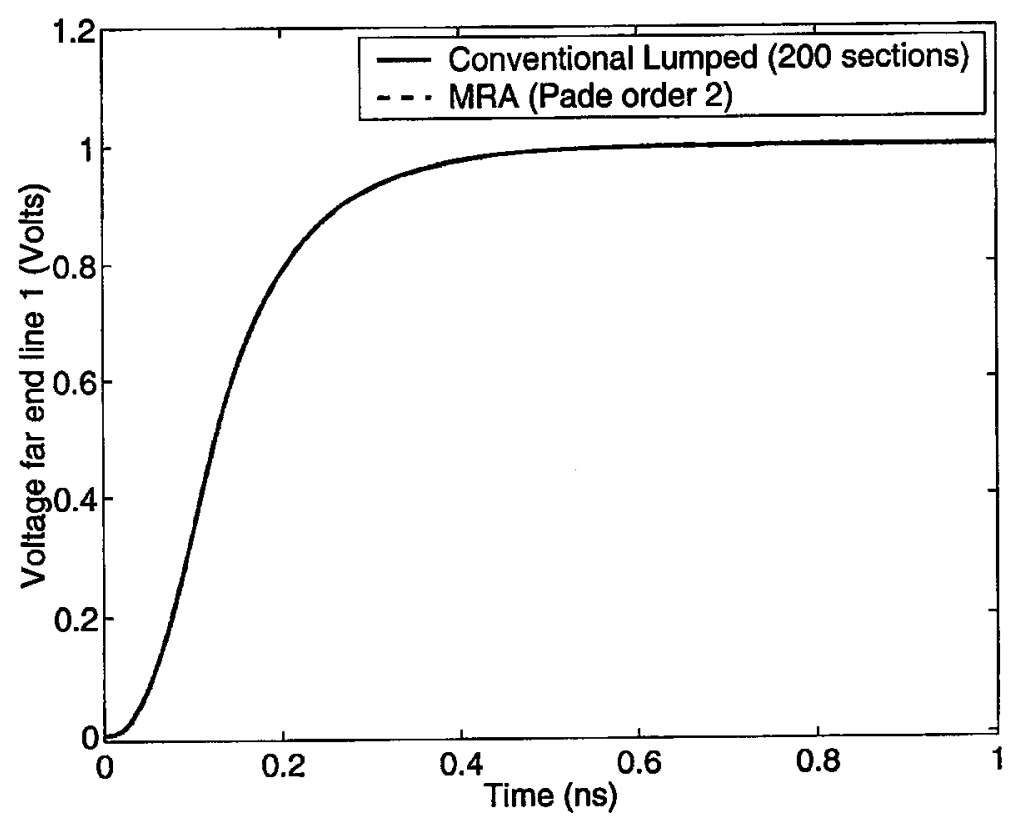

a) Far end line 1

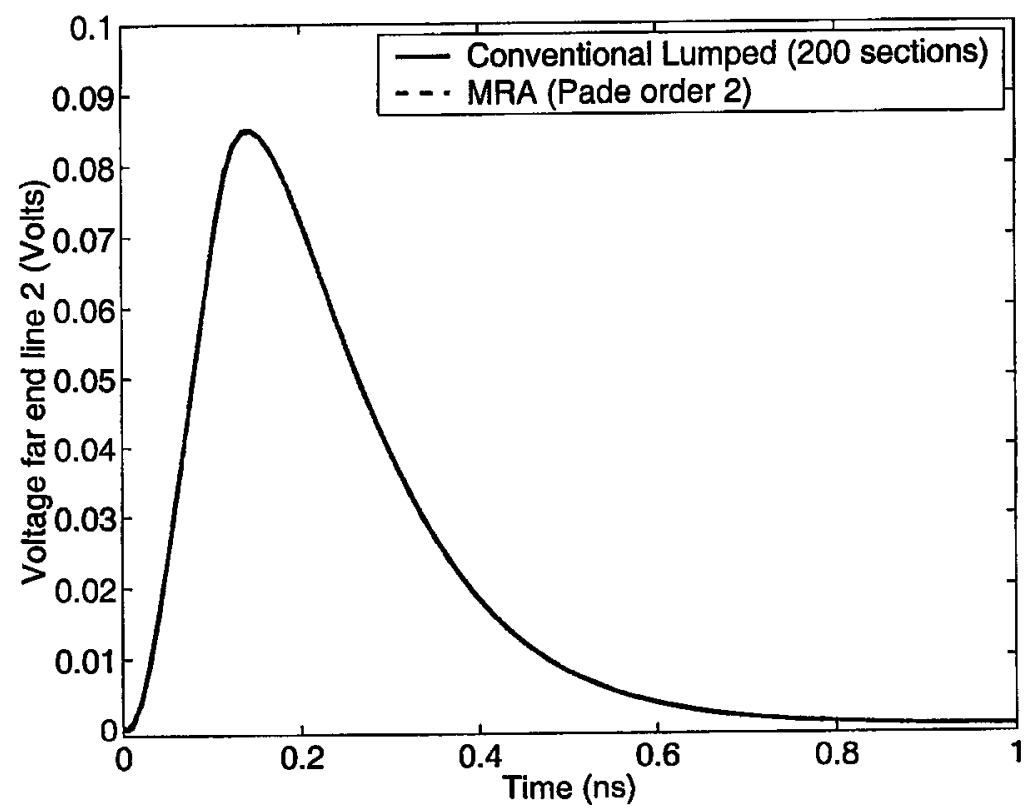

b) Far end line 2

Figure 4.13 Transient response (Example 4.1) 


\subsubsection{Example 4.2}

The coupled interconnect configuration proposed in [8] is shown in Figure 4.14. The p.u.l. parameters of the transmission lines are $R=\left[\begin{array}{ccc}3.448 & 0 & 0 \\ 0 & 3.448 & 0 \\ 0 & 0 & 3.448\end{array}\right] \frac{\Omega}{c m} L=\left[\begin{array}{ccc}4.976 & 0.765 & 0.152 \\ 0.765 & 4.976 & 0.765 \\ 0.152 & 0.765 & 4.976\end{array}\right] \frac{n H}{c m} C=\left[\begin{array}{ccc}1.082 & -0.197 & -0.006 \\ -0.197 & 1.082 & -0.197 \\ -0.006 & -0.197 & 1.082\end{array}\right] \frac{p F}{c m}$

and the length of each line is $5 \mathrm{~cm}$. In this example, the off diagonal p.u.l. resistances are zero since the return line is assumed to be lossless. The output frequency response obtained using the proposed macromodel is compared to the conventional lumped model and the 'Exact' response (obtained using eigen decomposition) in Figure 4.15. For their respective orders, both macromodels add equal number of variables to the MNA equations (63 variables for each transmission line). In terms of accuracy, the MRA macromodel matches the 'Exact' frequency response up to $6.5 \mathrm{GHz}$, while the conventional lumped model deviates from the 'Exact' response at $1 \mathrm{GHz}$. The transient response corresponding to an input pulse with rise/fall time $0.1 \mathrm{~ns}$ and a pulse width of $0.8 \mathrm{~ns}$ is shown in Figure 4.16. The response labeled IFFT refers to the inverse FFT of the 'Exact' response multiplied by the input frequency spectrum. The transient response of the proposed macromodel (order 7) shows good agreement with IFFT response.

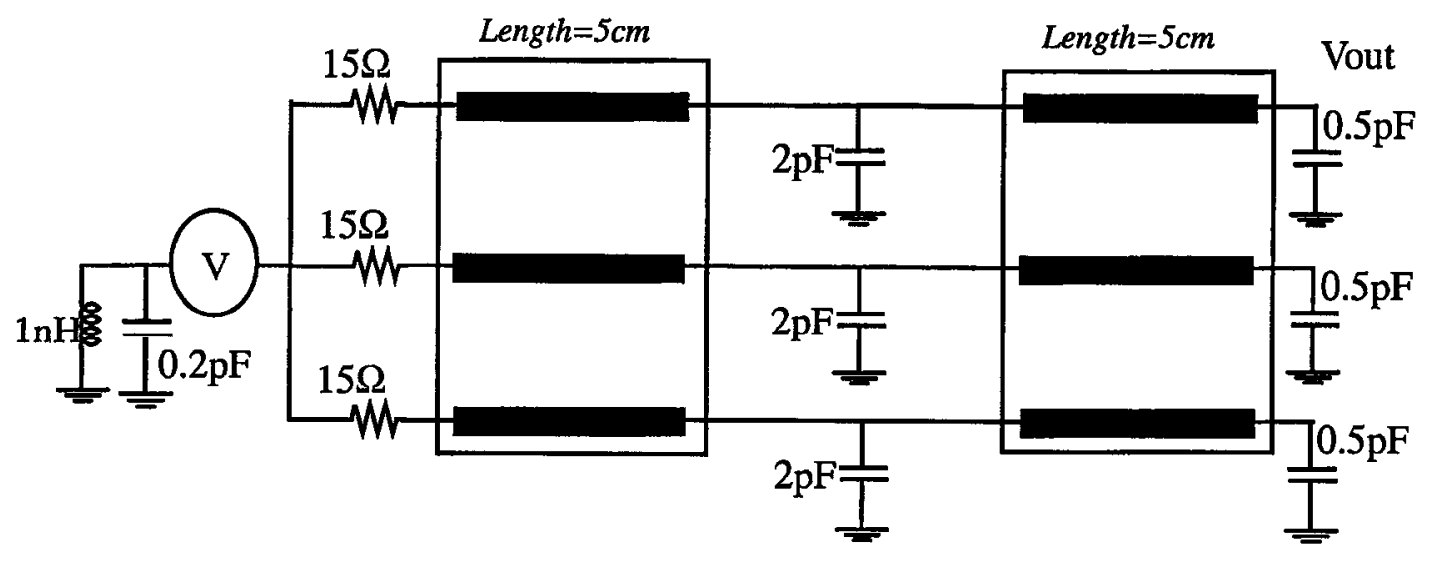

Figure 4.14 Coupled transmission line network (Example 4.2) 


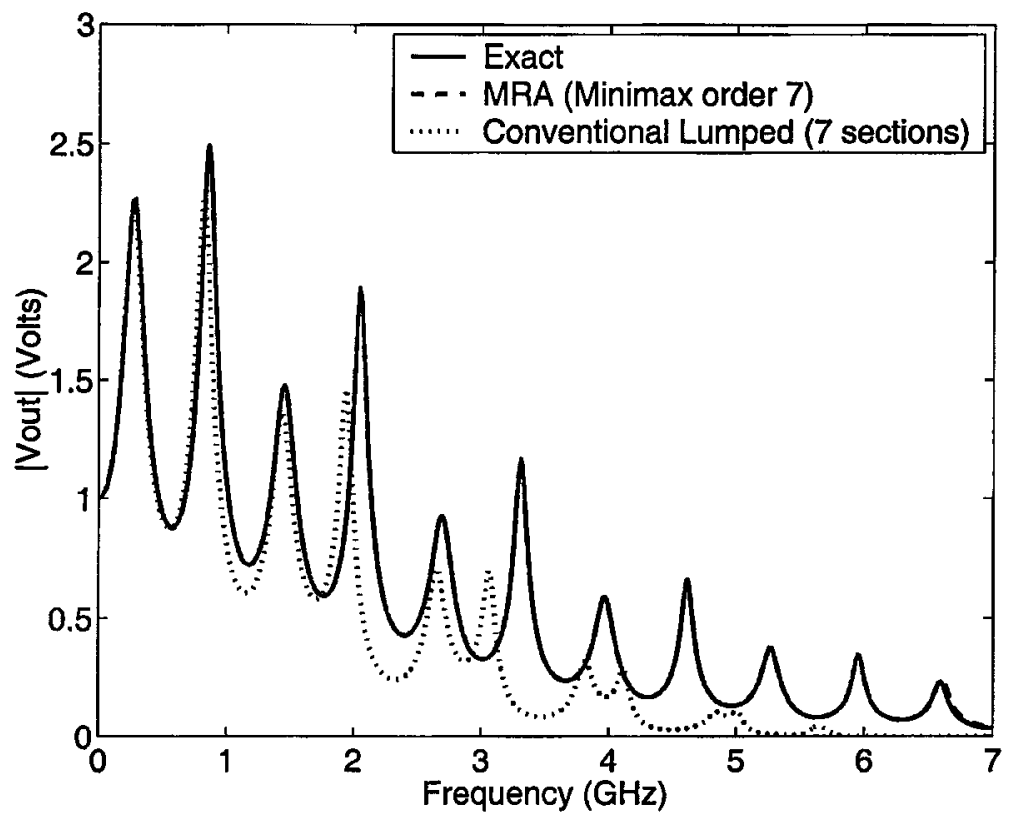

Figure 4.15 Frequency response (Example 4.2)

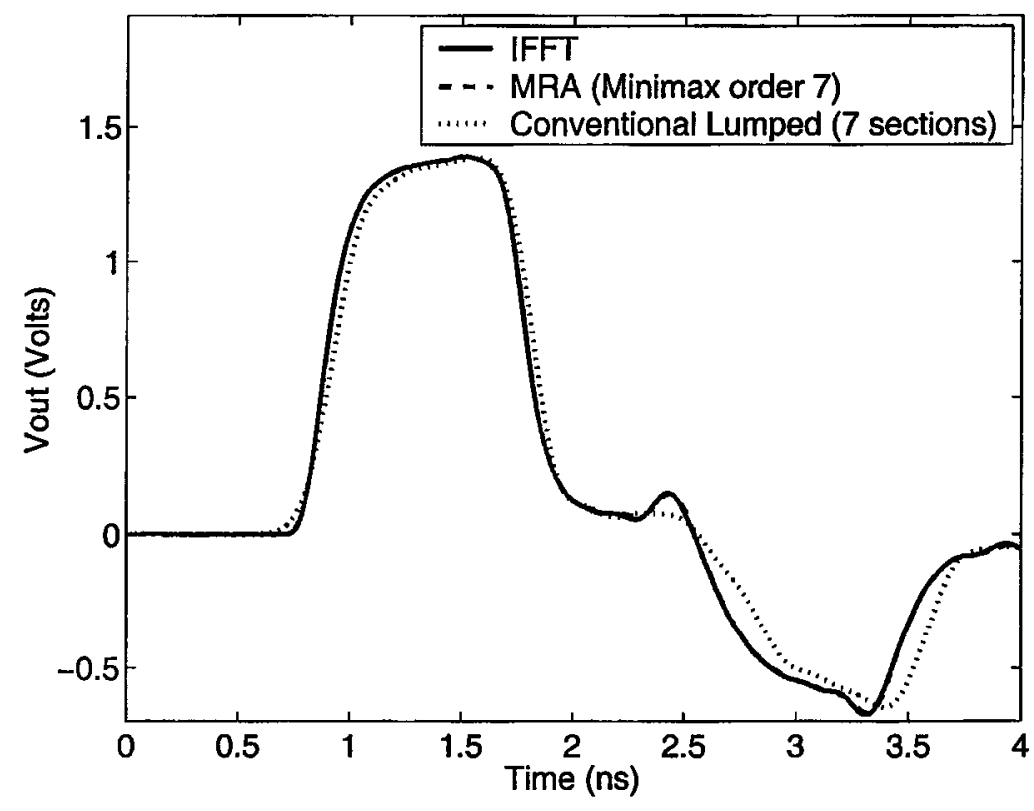

Figure 4.16 Transient response (Example 4.2) 


\subsubsection{Example 4.3}

In this example, a seven-transmission line network (Figure 4.17) with nonlinear terminations (CMOS inverters) is considered. The output transient responses at node $P 2$ and Vout corresponding to a trapezoidal pulse of amplitude $5 \mathrm{~V}$ with a rise/fall time of $0.1 \mathrm{~ns}$, pulse width of $5 \mathrm{~ns}$ and a period of $10 \mathrm{~ns}$ are shown in Figure 4.18. Both the MRA macromodel (order 5) and the conventional lumped segmentation model (33 sections) give similar results. However, the proposed method introduces 14 new variables for each transmission line, whereas the conventional lumped model introduces 98 ( $86 \%$ savings). In terms of CPU time, the proposed method required 7 seconds to simulate the entire nonlinear network while the conventional lumped model required 33 seconds.

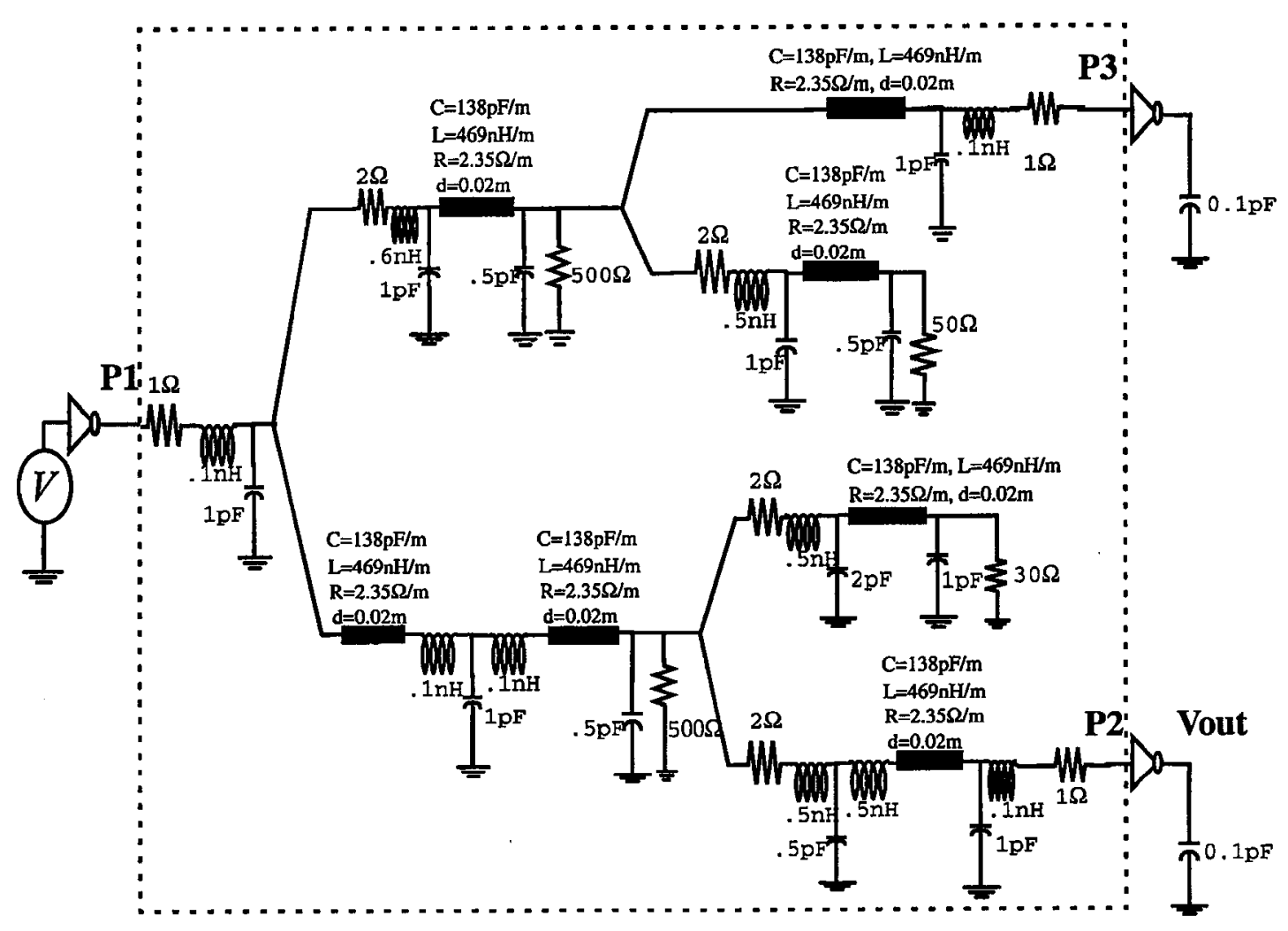

Figure 4.17 Seven transmission line network (Example 4.3) 


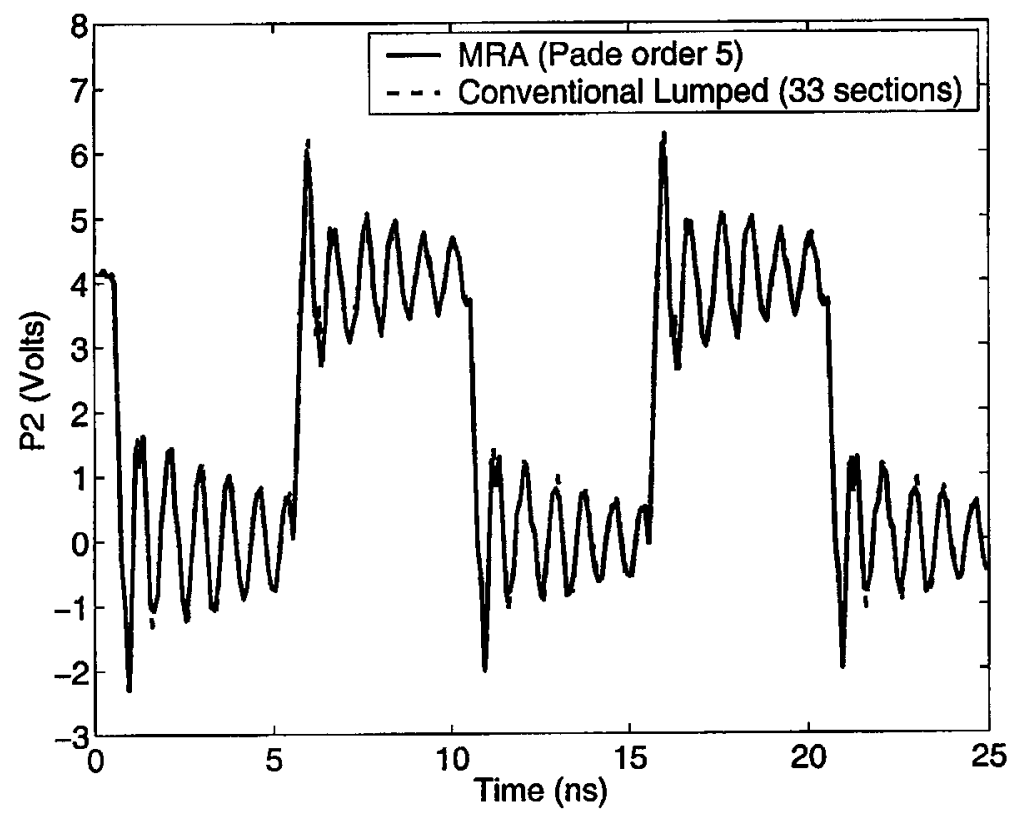

a) At node P2

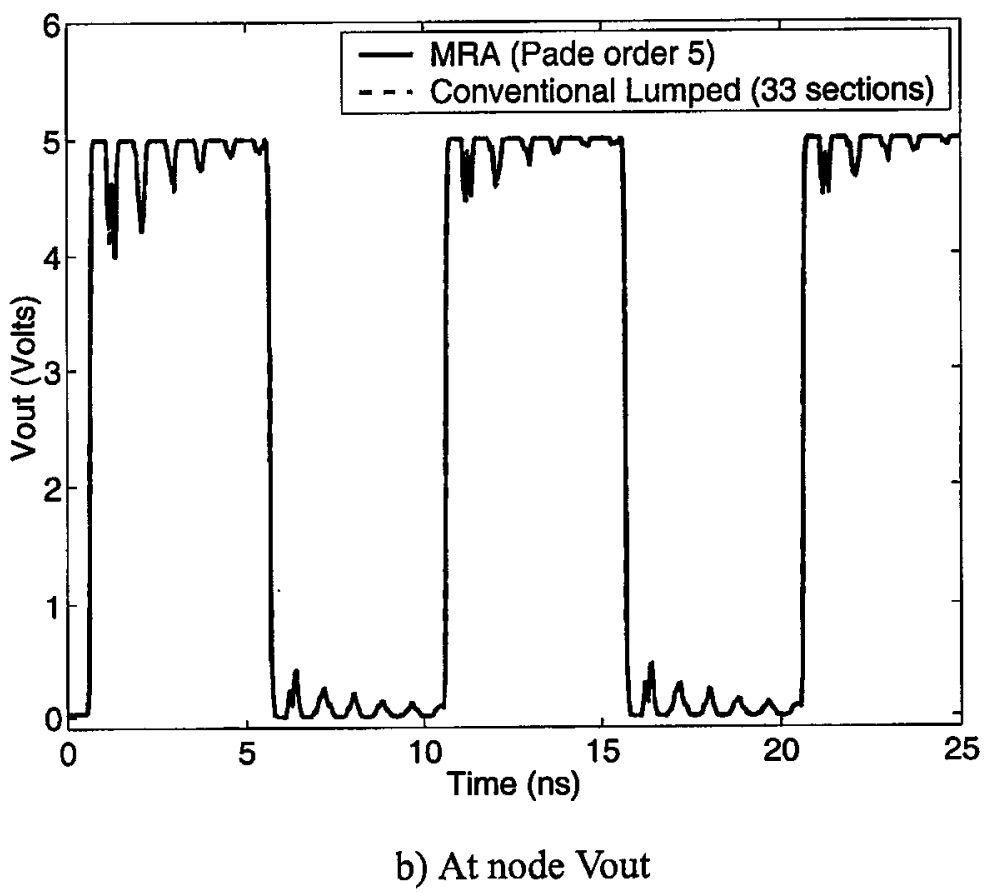

Figure 4.18 Transient response of nonlinear network (Example 4.3) 


\subsubsection{Example 4.4}

A lossy microstrip structure with three-conductors (two signal conductors plus one reference conductor) is used to validate the proposed algorithm for incident field coupling analysis (Figure 4.19). In the first part of this example, the structure is analyzed in the presence of incident fields with resistive loads (Figure 4.20). In the second part of the example, the structure is terminated by nonlinear elements and transient analysis in the presence of field coupling is carried out.

The incident field is a TM-polarized plane wave illuminating the lines at $\theta=45^{\circ}$ and $\phi=0^{0}$ angles. The p.u.l. parameters of the structure are

$$
\begin{array}{rlrl}
L & =\left[\begin{array}{ll}
623.3 & 132.9 \\
132.9 & 623.3
\end{array}\right] \mathrm{nH} / \mathrm{m} & C=\left[\begin{array}{cc}
50.18 & -7.359 \\
-7.359 & 50.18
\end{array}\right] \mathrm{pF} / \mathrm{m} \\
R & =\left[\begin{array}{ll}
42.73 & 2.401 \\
2.401 & 42.73
\end{array}\right] \Omega / m & G=\left[\begin{array}{cc}
2.514 & -0.2865 \\
-0.2865 & 2.514
\end{array}\right] \mathrm{J} / \mathrm{m}
\end{array}
$$

Initially, a SPICE model is generated for the equivalent sources (Padé Order=12) as explained in section 4.4.2. For a transient simulation in SPICE, a time-domain envelope waveform of a Gaussian pulse shape with amplitude of $1 \mathrm{KV} / \mathrm{m}$ and a bandwidth of 10 $\mathrm{GHz}$ is used. The pulse is defined in a piecewise manner in SPICE. For validating the results, coupling to the test structure is also obtained in the frequency domain through conventional analysis [2]. The result is multiplied with the spectrum of the Gaussian pulse and the time domain response is obtained by Inverse Fast Fourier transform (IFFT). Figure 4.21 shows the comparison of results from both the approaches. As seen the results are indistinguishable.

It is to be noted that the incident field in free space will be modified due to the dielectric slab surrounding the traces. The equivalent current sources for this case can be modified using the algorithm described in [41] without sacrificing the semi-analytic form of computational procedure presented in this chapter.

The microstrip structure of Figure 4.19 is connected to a CMOS inverter circuit for nonlinear simulation (Figure 4.22). In addition to external fields, the circuit is driven by a voltage source having a rectangular waveform with amplitude of 5 volts, switching transi- 
tion time of $0.1 \mathrm{~ns}$ and duty cycle of $2 \mathrm{~ns}$. The objective of this example is to predict the effect of incident fields on the circuit operation. For this purpose, (a) the circuit is initially analyzed with no external field disruption, (b) with the effect of external fields, using the proposed SPICE model. Both results are plotted in Figure 4.23. It is to be noted that, it is difficult to handle the incident field analysis of such lossy multi-conductor structures with nonlinear terminations using conventional frequency-domain approach.
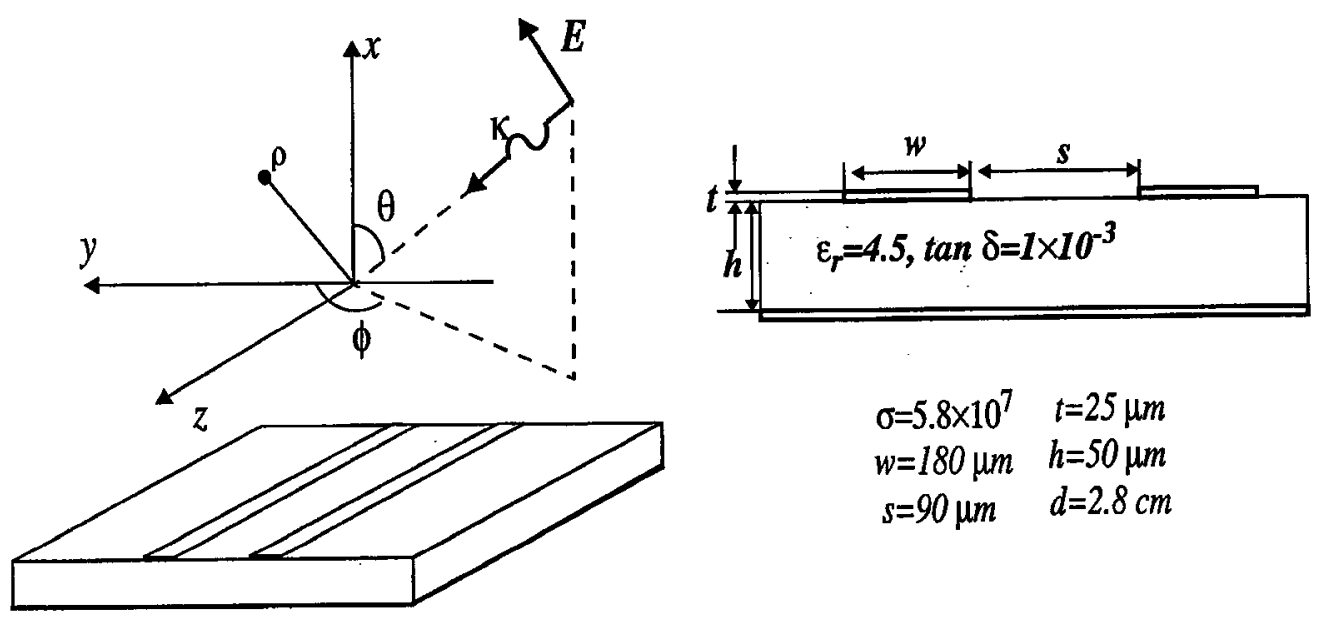

$$
\begin{array}{cc}
\sigma=5.8 \times 10^{7} & t=25 \mu \mathrm{m} \\
w=180 \mu \mathrm{m} & h=50 \mu \mathrm{m} \\
s=90 \mu \mathrm{m} & d=2.8 \mathrm{~cm}
\end{array}
$$

Figure 4.19 Coupled Lossy Microstrip Line

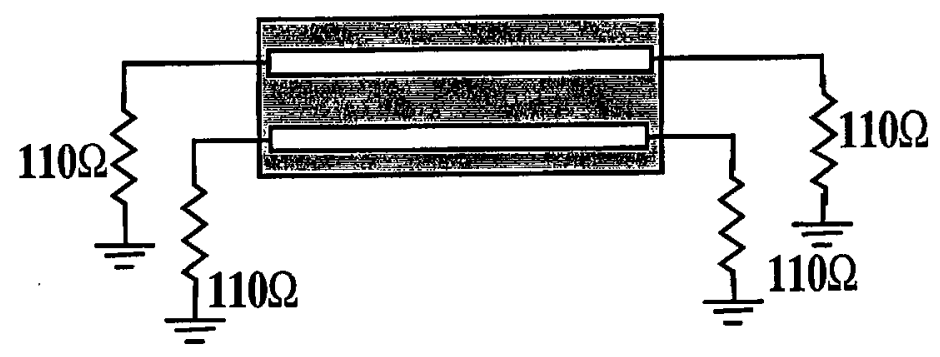

Figure 4.20 Linear test circuit 


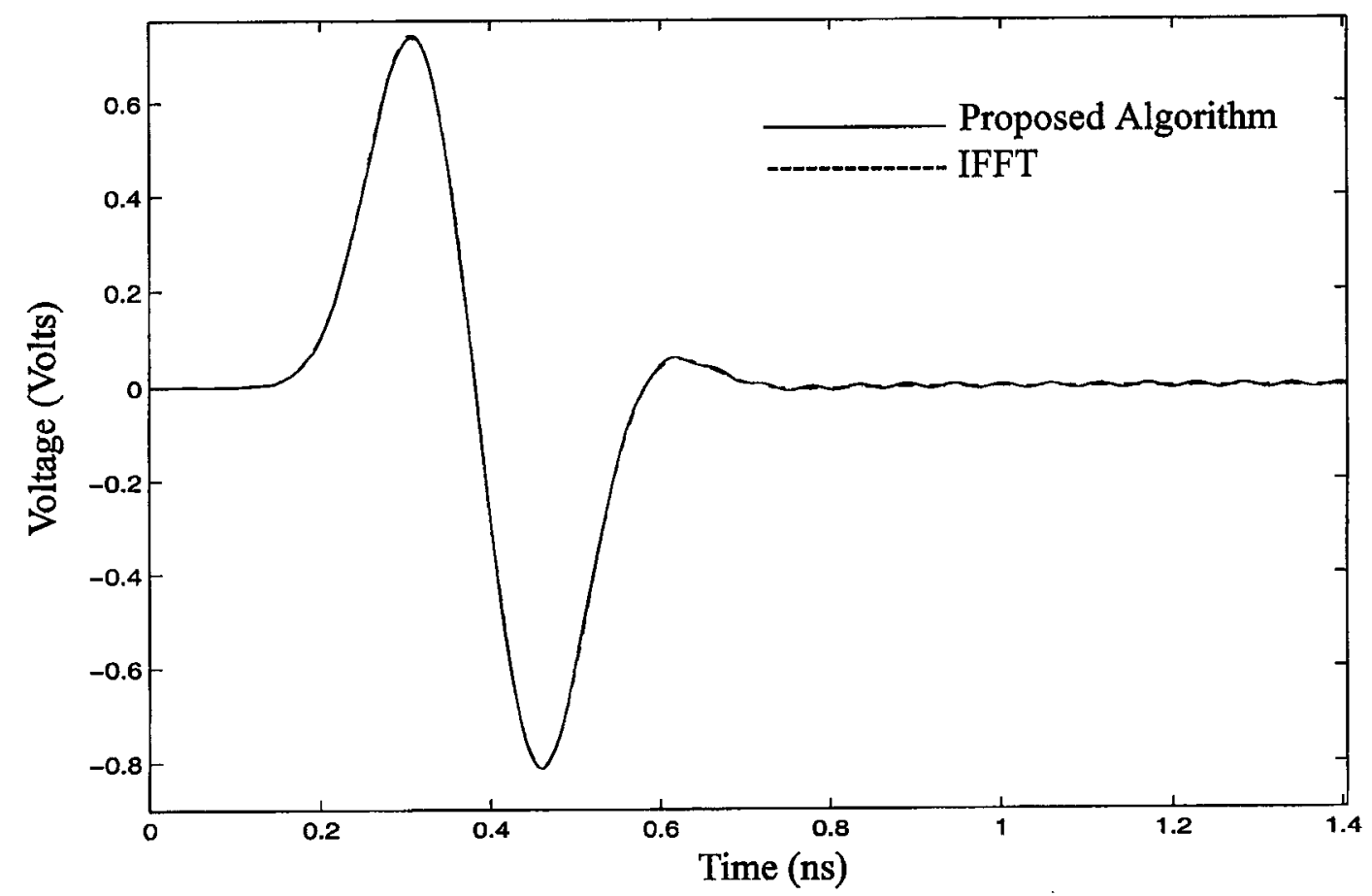

Figure 4.21 Coupled Voltage Waveform for The Linear Circuit

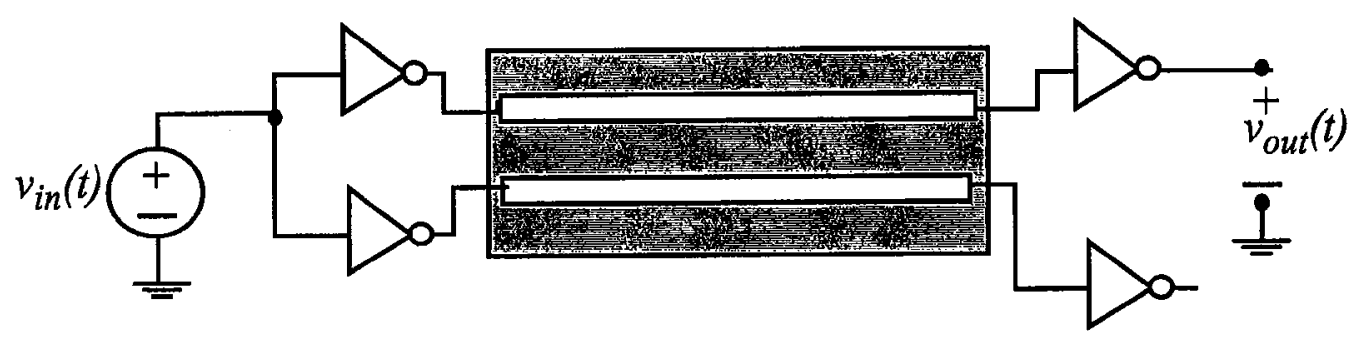

Figure 4.22 Coupled transmission-line with non-linear terminations (Example 8.1) 


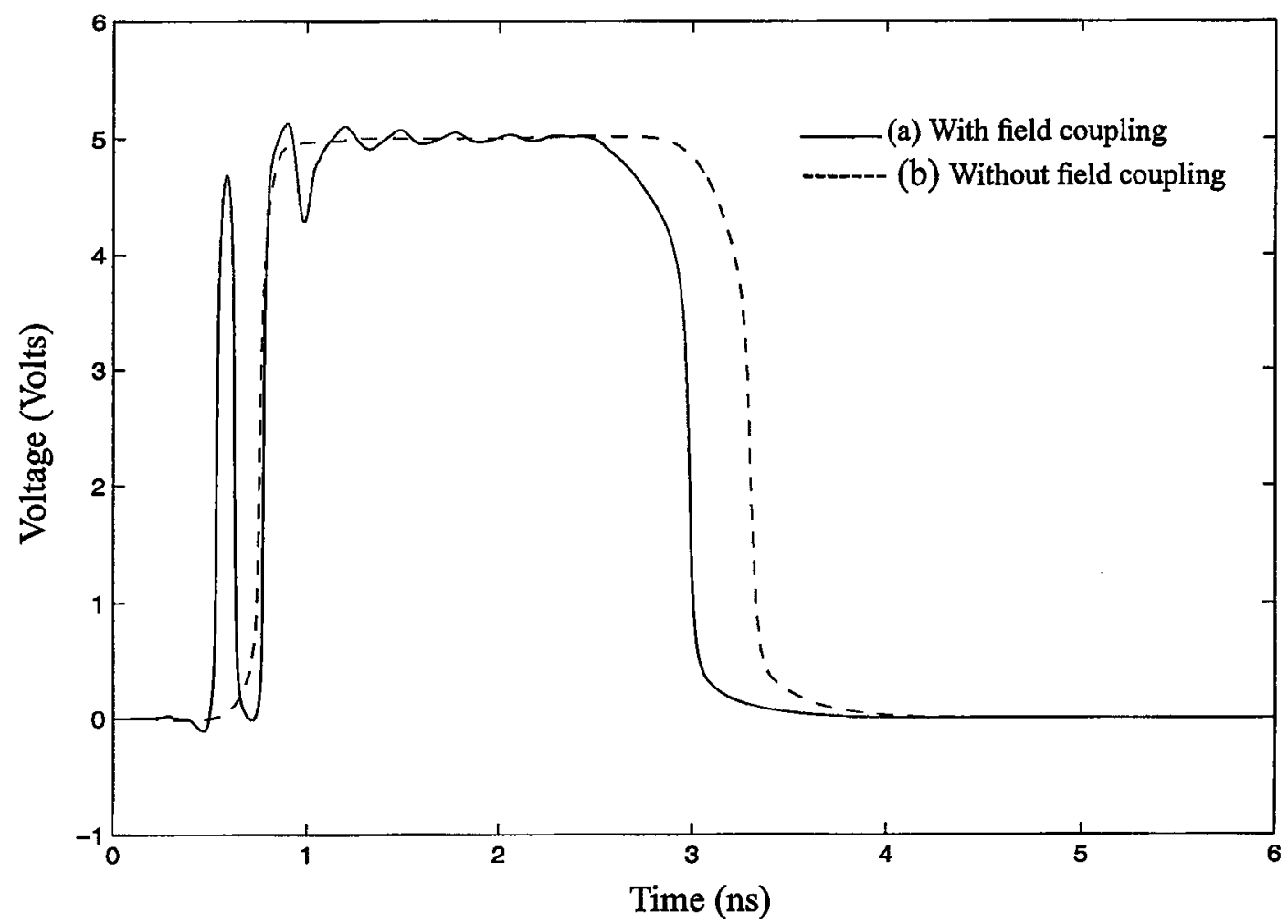

Figure 4.23 Output Waveform of The Nonlinear Test Circuit 


\section{Chapter 5}

\section{Frequency-Dependent Parameters}

\subsection{Introduction}

Analysis of interconnects with frequency-dependent parameters is important for analyzing high-speed applications. It is shown in [2], [34]-[35], [87] that neglecting these effects leads to significant errors in simulation results. Interconnects with imperfect conductors and inhomogeneous dielectrics create an electromagnetic field structure that is not pure transverse electromagnetic (TEM) mode. The mode of propagation of such lines is referred to as quasi-TEM. An important consequence of quasi-TEM is that the conductivity of imperfect conductors and the effective wave velocity of inhomogeneous medium are functions of frequency. To accurately model this behaviour, transmission line models with frequency-dependent parameters are required. In this chapter, the matrix rational approximation (MRA) macromodel is used for passive macromodeling of transmission lines with frequency-dependent parameters [52].

The organization of this chapter is as follows. Section 5.2 provides some background information on the typical behaviour of the per-unit-length (p.u.1.) parameters as functions of frequency. A technique to model frequency-dependent parameters while preserving passivity of the MRA macromodel is described in section 5.3. Section 5.4 provides the time-domain formulation of the MRA macromodel for transmission lines with frequency- 
dependent parameters. Numerical examples to illustrate the validity of the proposed method are provided in section 5.5 .

\subsection{Behaviour of Per-Unit-Length Parameters}

As frequency increases, an electric field is induced which causes the current distribution of conductors to vary. This causes the p.u.l. resistance and inductance to change as frequency increases. These changes are classified into three parts: proximity, edge and skin effect.

The edge and proximity effects influence the p.u.l. resistance and inductance at the medium frequency range. At low frequencies the p.u.l. resistance and inductance are practically constant. As frequency increases, the edge effect causes current to concentrate at the sharp edges of the conductor, thus increasing the resistance. This affects both the signal and ground conductor, however, the current concentration is more pronounced on the signal conductors. The proximity effect causes current to concentrate in sections of the ground plane that are near to the signal conductors. This reduces the magnetic field in the conductors, which causes the inductance to drop. The proximity effect also increases the p.u.l. resistance since more current is crowded in the ground plane near the signal conductors. The skin effect occurs at the high frequency region and influences both ground and signal conductors. As frequency increases, current begins to concentrate in a thin layer at the conductor surface. The thickness of the layer is related to the skin depth, and decreases as frequency increases. This causes the resistance to be directly proportional to the square root of frequency. The magnetic field inside the ground and signal conductors are also reduced at high frequencies. This causes the p.u.1. inductance to drop even further. At higher frequencies, the inductance contribution due to the magnetic field inside the ground and signal conductors becomes negligible and only the magnetic field external to the conductors contribute to the total inductance. This causes the inductance to decrease and approach a constant value.

At high frequencies, it may also be necessary to model the p.u.l. conductance and ca- 
pacitance as functions of frequency. The losses due to the conductive and polarization properties of the dielectric increase as frequency increases. This causes the conductance parameter to increase with frequency. In addition, the capacitance parameter also changes with frequency due to change in permittivity and losses of the dielectric material, however, these variations are usually vary small.

The frequency plots of the p.u.1. parameters of a stripline in Figure 5.1 are shown in Figure 5.2 and Figure 5.3. These plots show the typical behavior of the p.u.l. parameters. Both the resistance and inductance are essentially constant at low frequencies. As frequency increases, the edge and proximity effects cause the resistance to increase and the inductance to decrease. At higher frequencies the skin effect causes the resistance to be directly proportional to the square root of frequency, while the inductance approaches a constant value (Figure 5.2). The loss tangent of the dielectric medium causes the p.u.l. conductance to increase as frequency increases, while the variation of the p.u.l. capacitance decreases slightly (Figure 5.3).

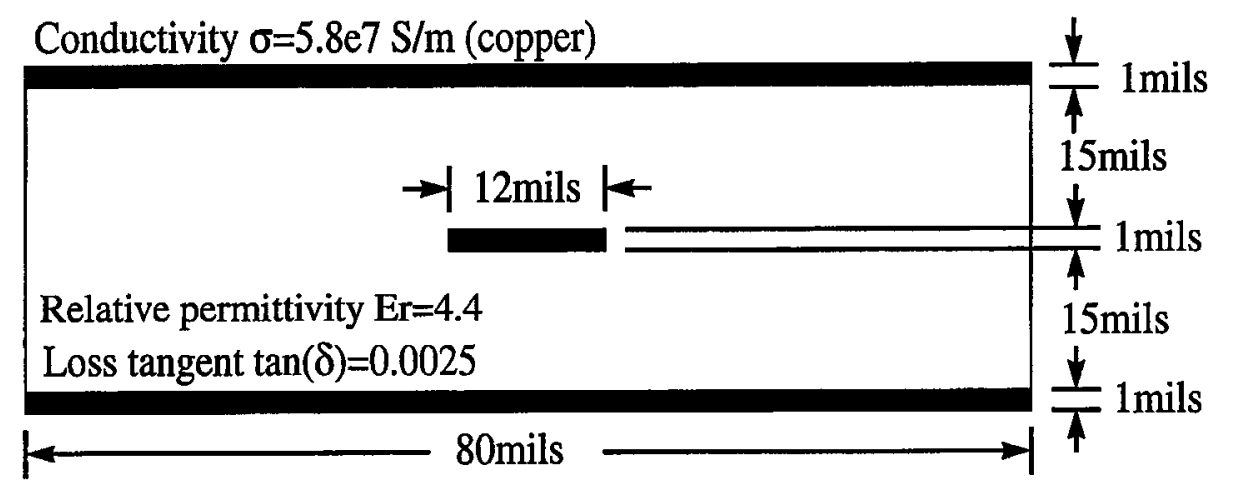

Figure 5.1 Cross-sectional geometry and dimensions of stripline interconnect 


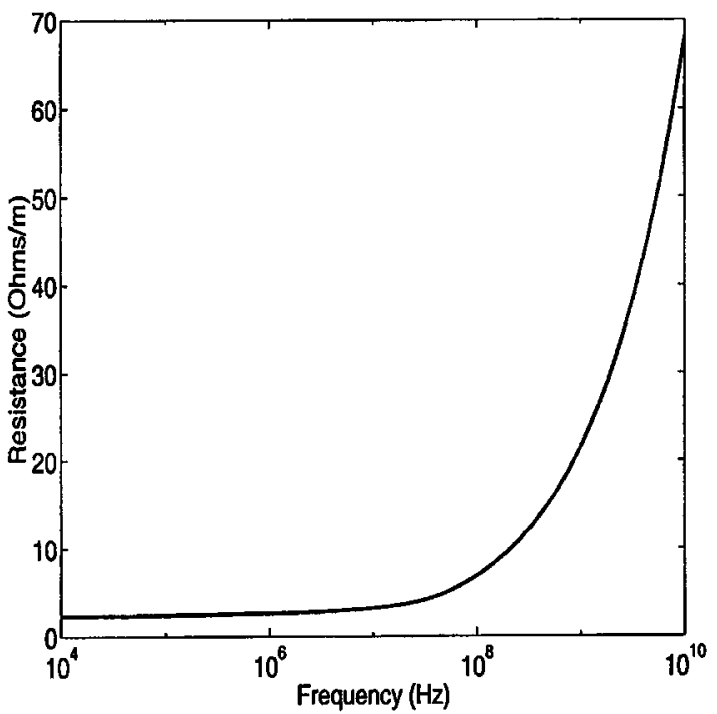

a) Resistance versus frequency

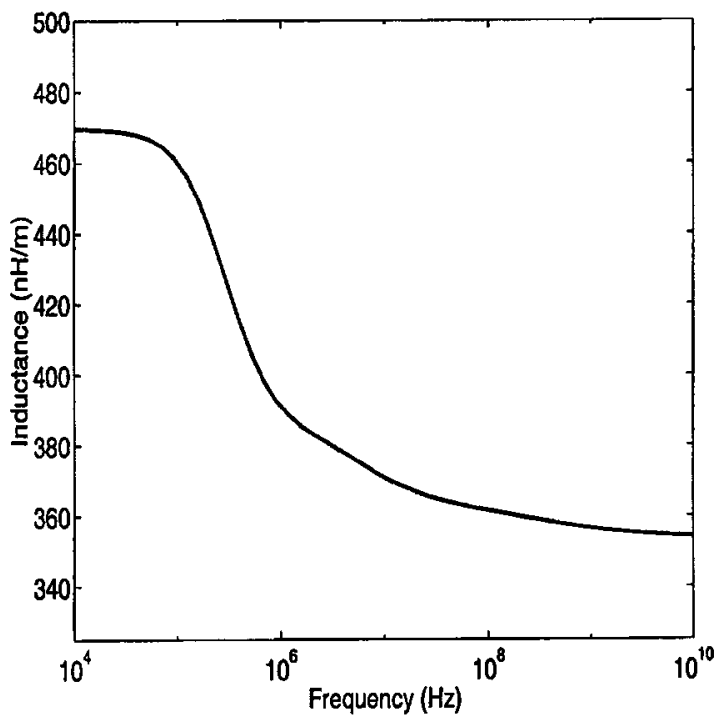

b) Inductance versus frequency

Figure 5.2 P.u.l. resistance and inductance versus frequency

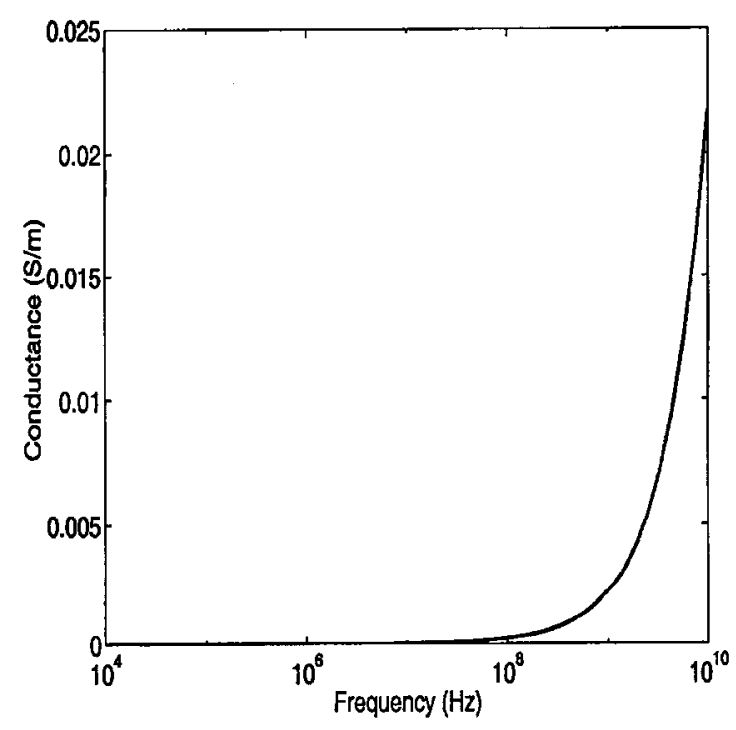

a) Conductance versus frequency

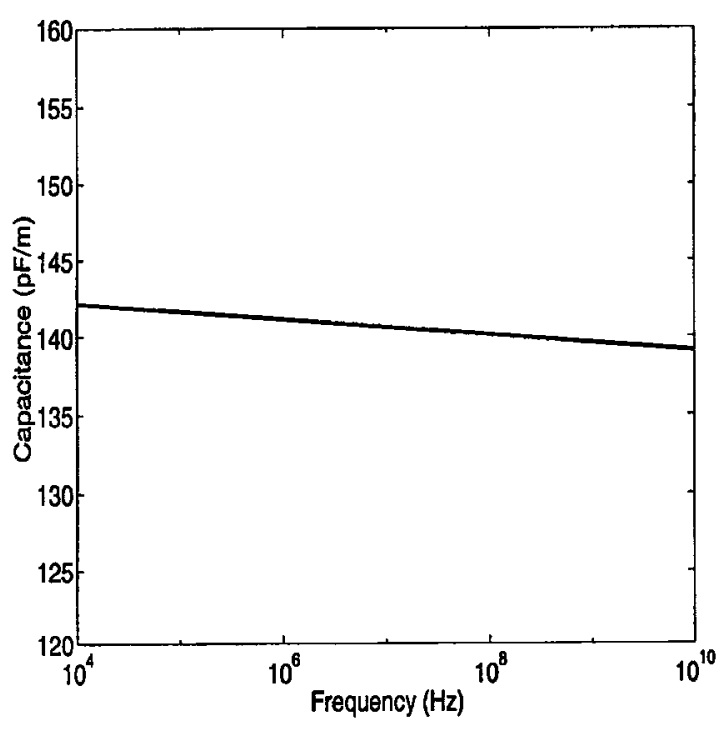

b) Capacitance versus frequency

Figure 5.3 P.u.l, conductance and capacitance versus frequency 


\subsection{Modeling of Frequency-Dependent Parameters}

The passivity theorem of Chapter 3 requires the functions $R(s)+s L(s)$ and $\boldsymbol{G}(s)+s C(s)$ to be positive-real. The extracted p.u.l. parameters of transmission lines are nonnegative matrices at each frequency point of operation, hence the functions formed by $R(s)+s L(s)$ and $G(s)+s C(s)$ are positive real [2], [23]. However, the p.u.l. parameters are often characterized by tabulated data obtained from measurements, empirical formulae or electromagnetic simulation [2], [82]-[87]. To preserve the passivity of the MRA macromodel it is necessary to approximate the tabulated data with positive-real functions. For this purpose, a scheme is presented to construct positive-real rational functions from the tabulated data of the p.u.l. parameters.

Frequency-dependent parameters are often characterized by rational functions [8], [26], [34]-[35]. Such structures can easily be incorporated into the proposed macromodel since the model itself is in a rational form. Let the frequency-dependent line parameters be modeled as

$$
\begin{aligned}
& \tilde{\boldsymbol{a}}(s)=\boldsymbol{R}(s)+s \boldsymbol{L}(s)=\frac{N_{Z}}{D_{Z}}=\frac{\boldsymbol{f}_{k} s^{k}+\ldots+\boldsymbol{f}_{0}}{w_{k-1} s^{k-1}+\ldots+1} \\
& \tilde{\boldsymbol{b}}(s)=\boldsymbol{G}(s)+s \boldsymbol{C}(s)=\frac{\boldsymbol{N}_{Y}}{D_{Y}}=\frac{\boldsymbol{g}_{k} s^{k}+\ldots+\boldsymbol{g}_{0}}{h_{k-1} s^{k-1}+\ldots+1}
\end{aligned}
$$

where $N_{Z}$ and $N_{Y}$ are polynomial matrices; $D_{Z}$ and $D_{Y}$ are scalar polynomials.

Directly forcing the rational function of $\tilde{\boldsymbol{a}}(s)$ and $\tilde{\boldsymbol{b}}(s)$ to be positive real requires nonlinear constraints. However, nonlinear constraints are usually difficult to implement as well as suffers from convergence problems [92]. In order to overcome this difficulty, rational functions are represented by passive circuit elements, which simplify the formulation to linear constraints [77]-[79]. This approach has practical advantages since most commercial circuit simulators are capable of optimizing linear networks using sensitivity analysis [93]-[94]. Also, the method is extended to include the frequency-dependency of mutual parameters (such as $R_{12}, L_{12}$ etc.). 
For the sake of simplicity, the discussion will focus on two-conductor transmission lines structures and then extend to multi-conductor transmission lines (MTLs). The circuit configurations of Figure 5.4 are often used to model the frequency-dependent parameters of the resistance and inductance [2], [4], [34]. Both the RL canonical and RL Foster-like configurations are capable of capturing the frequency response of the p.u.l. resistance and inductance. In the proposed algorithm, the frequency-dependent data of $R(s)+s L(s)$ is matched to the input impedance of a passive network. By ensuring each element is nonnegative, the resulting network remains passive and the rational function formed by the input impedance is guaranteed to be positive-real [77]-[79]. To capture the frequency response of the conductance and capacitance the circuit topology is composed of resistors and capacitors. Figure 5.5 shows two circuit topologies capable of capturing the frequency response of the p.u.l. conductance and capacitance. The frequency-dependent data of $G(s)+s C(s)$ is matched to the input admittance of a passive network to obtain a positivereal rational function.

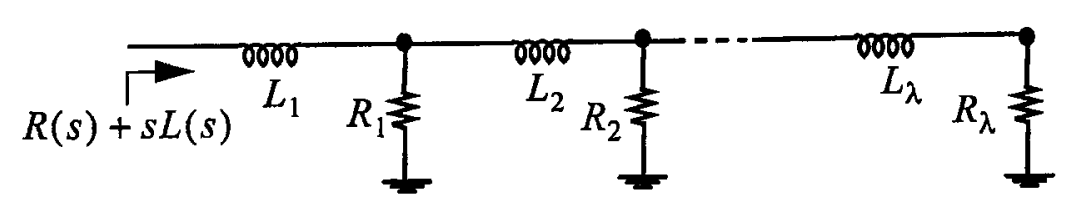

a) RL canonical circuit configuration

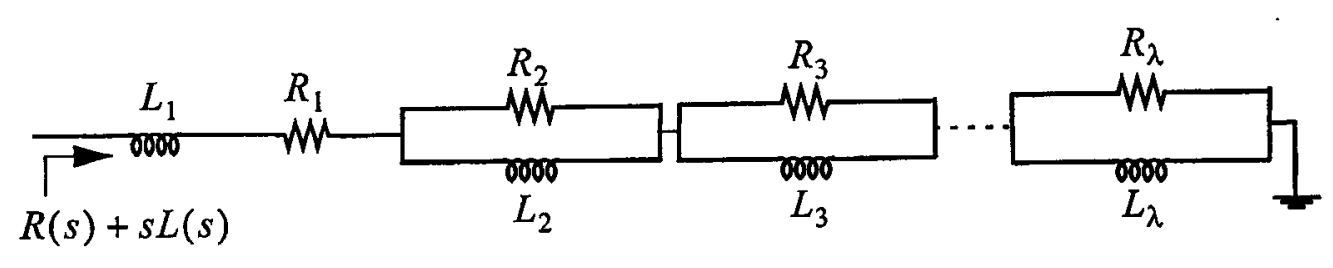

b) RL Foster-like circuit configuration

Figure 5.4 Modeling of $R(s)+s L(s)$ 


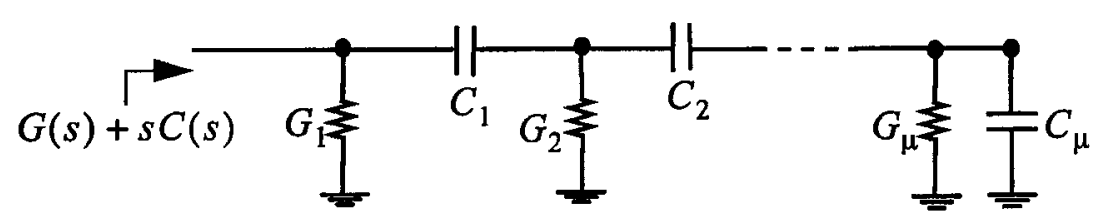

a) RC canonical circuit configuration

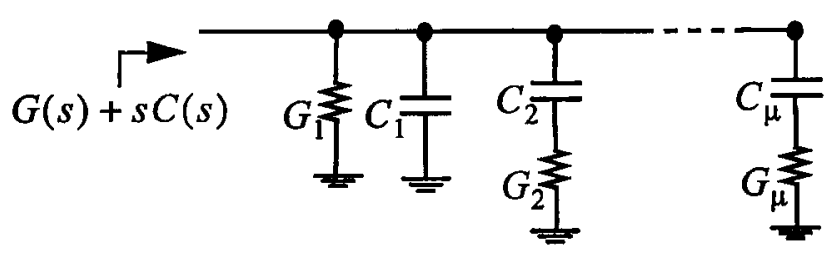

b) RC Foster-like circuit configuration

Figure 5.5 Modeling of $G(s)+s C(s)$

For MTLs, a similar strategy is used to construct positive real rational functions. The function $\boldsymbol{R}(s)+s \boldsymbol{L}(s)$ can be expressed as,

$$
\tilde{a}(s)=\boldsymbol{R}(s)+s L(s)=R(s)+s\left(L_{i}(s)+L_{e}\right)
$$

where $L_{i}(s)$ is frequency-dependent and is due to the magnetic flux internal to the conductors; $\boldsymbol{L}_{\boldsymbol{e}}$ is a constant nonnegative definite matrix and is due to the magnetic flux external to the conductors [2]. To model the resistance and inductance parameters the circuit topology is composed of resistors, inductors and ideal transformers. Figure 5.6 shows an example circuit topology to model a four-conductor line (three transmission-lines and one reference line). The general form of the rational function matrix becomes 


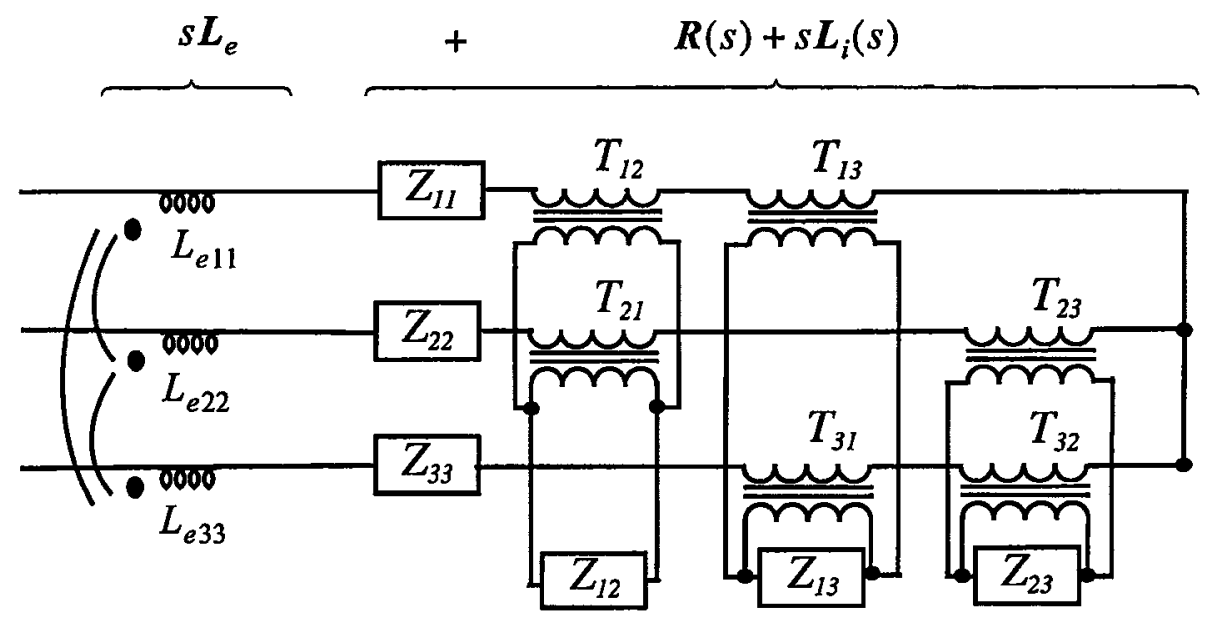

a) Example circuit topology for $R(s)+s L(s)$ for MTL

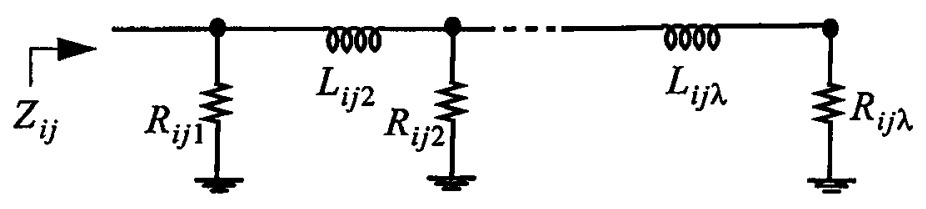

b) Circuit representation of $Z_{i j}$, RL canonical configuration

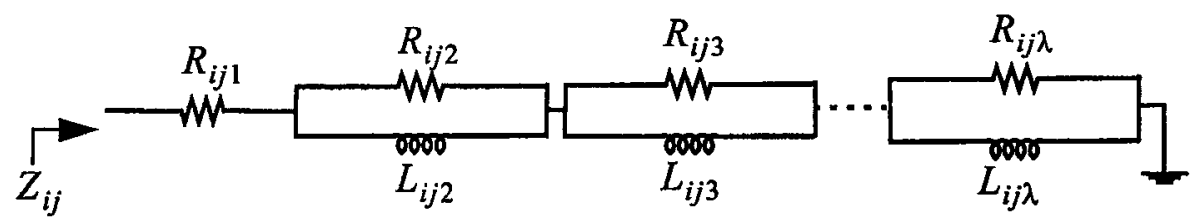

c) Circuit representation of $Z_{i j}$, RL Foster-like circuit configuration

Figure 5.6 Modeling of $\boldsymbol{R}(s)+s L(s)$ for MTL 


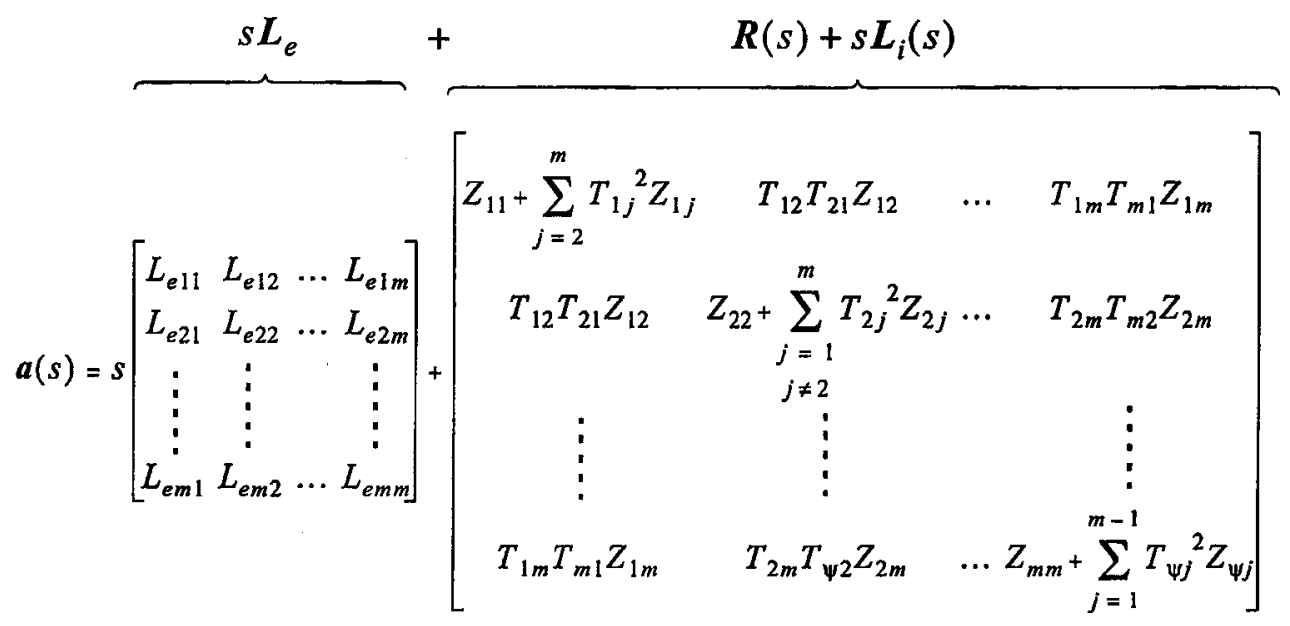

where $T_{i j}$ is the turn ratio of the ideal transformer and $Z_{i j}$ represents the impedance of the circuit given by Figure $5.6 \mathrm{~b}$ or Figure 5.6c. The matrix configuration of (5.3) represents the Z-parameters of the circuit for the general case. Since the matrix $L_{e}$ is nonnegative definite and imposing the constraints that all resistors and inductors are all positive makes (5.3) positive-real [77]-[79]. To model the conductance and capacitance parameters the circuit topology is composed of resistors and capacitors. Figure 5.7 shows an example circuit topology to model $G(s)+s C(s)$ for a four-conductor line. The general form of the rational function matrix becomes

$$
\tilde{\boldsymbol{b}}(s)=\left[\begin{array}{cccc}
\sum_{j=1}^{m} Y_{1 j} & -Y_{12} & \ldots & -Y_{1 m} \\
-Y_{21} & \sum_{j=1}^{m} Y_{2 j} & \ldots & -Y_{2 m} \\
\vdots & \vdots & & \vdots \\
-Y_{m 1} & -Y_{m 2} & \ldots & \sum_{j=1}^{m} Y_{m j}
\end{array}\right]
$$

where $Y_{i j}$ represents the admittance of the circuit given by Figure $5.7 \mathrm{~b}$ or Figure $5.7 \mathrm{c}$. The matrix configuration of (5.4) represents the Y-parameters of the circuit for the general case. Imposing the constraints that all resistors and capacitors are nonnegative makes the rational matrix of (5.4) positive-real [77]-[79]. 


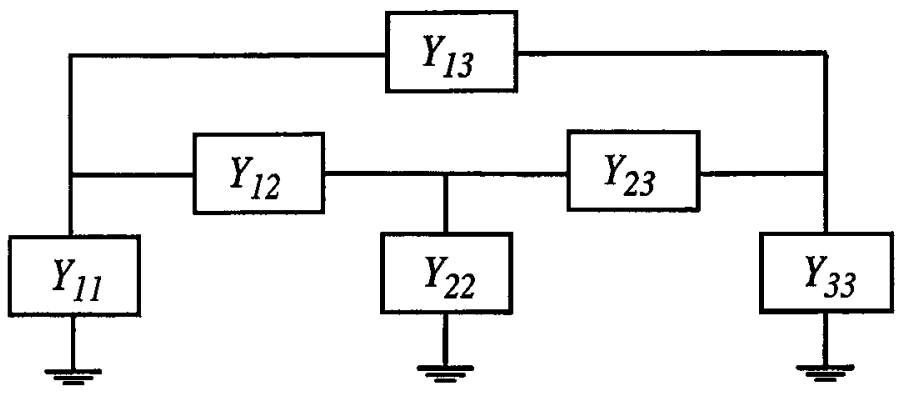

a) Example circuit topology for $G(s)+s C(s)$ for multi-conductor interconnect

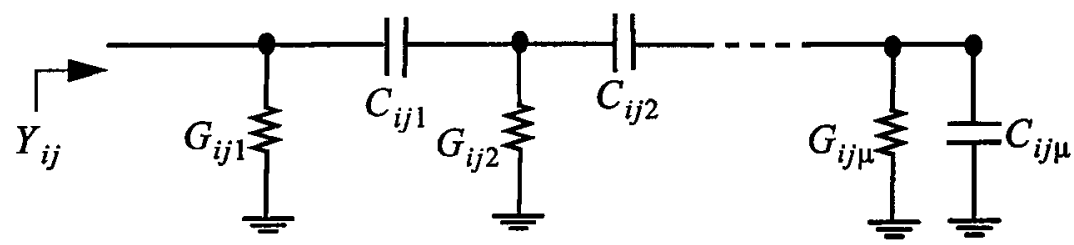

b) Circuit representation of $Y_{i j}$, RC canonical configuration

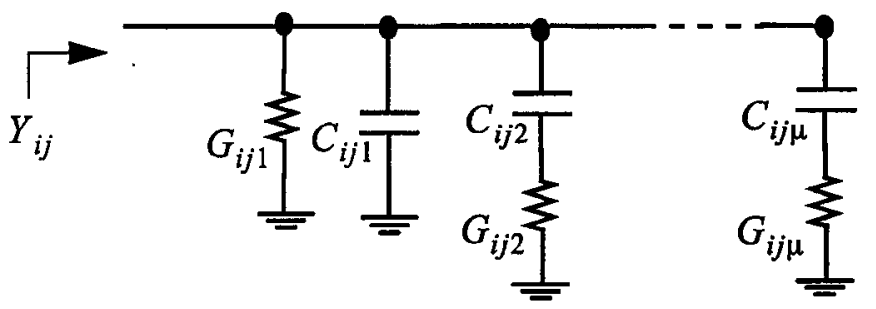

c) Circuit representation of $Y_{i j}$, RC Foster-like circuit configuration

Figure 5.7 Modeling of $G(s)+s C(s)$ for MTL 


\subsection{Circuit Realization for Frequency-Dependent Parameters}

To obtain a passive macromodel for transmission lines with frequency-dependent parameters, the p.u.l. parameters must be fitted to positive real rational functions (Theorem 3.1 of Chapter 3). A scheme to fit the p.u.l. parameters to positive real functions using passive circuit networks was described in the previous section. In this section, the time-domain formulation of the MRA is described for transmission lines with frequency-dependent parameters.

In Chapter 4, the time-domain macromodel is derived by writing MRA in terms of products of low order rational functions, which can be realized in terms of circuit elements. The Y-parameters of each subsection are described by (4.13) and (4.14), as

$$
\begin{gathered}
\boldsymbol{Y}_{11}=\boldsymbol{Y}_{22}=\frac{\left(x_{i}^{2}+y_{i}^{2}\right)}{4 x_{i}} a^{-1}+\frac{1}{4 x_{i}} b+x_{i}\left(a+\left(x_{i}^{2}+y_{i}^{2}\right) b^{-1}\right)^{-1} \\
\boldsymbol{Y}_{12}=\boldsymbol{Y}_{21}=\frac{-\left(x_{i}^{2}+y_{i}^{2}\right)}{4 x_{i}} a^{-1}-\frac{1}{4 x_{i}} b+x_{i}\left(a+\left(x_{i}^{2}+y_{i}^{2}\right) b^{-1}\right)^{-1} \\
Y_{11}=Y_{22}=\frac{\alpha_{0}}{2} a^{-1}+\frac{1}{2 \alpha_{0}} b \\
Y_{12}=Y_{21}=-\frac{\alpha_{0}}{2} a^{-1}+\frac{1}{2 \alpha_{0}} b
\end{gathered}
$$

where $\boldsymbol{a}=(\boldsymbol{R}(s)+s \boldsymbol{L}(s)) d, \boldsymbol{b}=(\boldsymbol{G}(s)+s \boldsymbol{C}(s)) d$ and $x_{i}, y_{i}$ and $\alpha_{0}$ are predetermined coefficients obtained from a scalar approximation of $e^{x}$, as described by (4.7)(4.12). Equation (5.5) and (5.6) describe the Y-parameters of the subsections derived from complex pole-zero pairs of (4.7) and the real pole-zero pair of (4.7), respectively. The time-domain macromodel for transmission lines with frequency-dependent parameters is derived from the rational fitting of the p.u.l. parameters and from the Y-parameter subsections of (5.5) and (5.6). 
The circuit realization of a two-conductor transmission line with frequency-dependent parameters that represent the Y-parameters of (5.5) and (5.6) are shown in Figure 5.8 and Figure 5.9. Figure 5.8 shows the topology corresponding to the complex pole-zero subsection of (5.5) and Figure 5.9 shows the topology for the real pole-zero subsection of (5.6). The realization of Figure 5.8 and Figure 5.9 are derived from the circuit topologies describing the p.u.l. parameters (Figure 5.4 and Figure 5.5) and by the Y-parameters of each subsection. The coefficient $\lambda$ and $\mu$ in Figure 5.8 and Figure 5.9 correspond to the number of sections required to model the frequency-dependent parameters. If $\lambda=\mu=1$, then the circuit realization of Figure 5.8 and Figure 5.9 is equivalent to the lossy (RLGC) transmission line realization in section 4.3.3.

The MRA realization of transmission lines with frequency-dependent parameters can also be extended to MTLs. An example of a three-conductor transmission line is shown in Figure 5.10 and Figure 5.11. Figure 5.10 shows the topology corresponding to the complex pole-zero subsection of (5.5) and Figure 5.11 shows the topology for the real polezero subsection of (5.6). Since the Y-parameters of each subsection are realized in terms of circuit elements, the corresponding modified nodal analysis (MNA) matrices can be expressed in terms of ordinary differential equation.

It should be noted that with the knowledge of the circuit configurations of the p.u.l. parameters (i.e. Figure 5.4 to Figure 5.7) the MRA macromodel for transmission lines with frequency-dependent parameters can be derived analytically in term of predetermined coefficients (i.e. $\alpha_{0}, x_{i}$ and $y_{i}$ ) and the circuit values of p.u.l. parameters. 


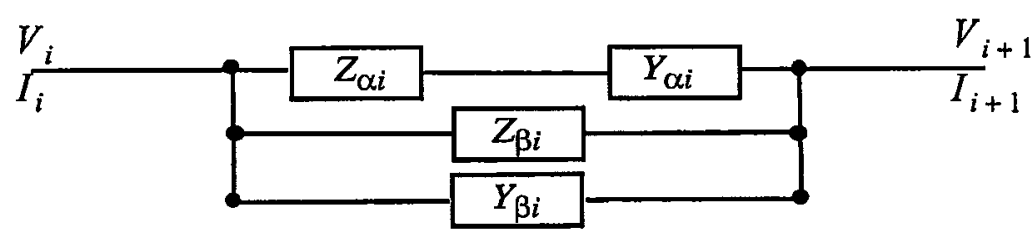

a) Complex pole-zero subsection

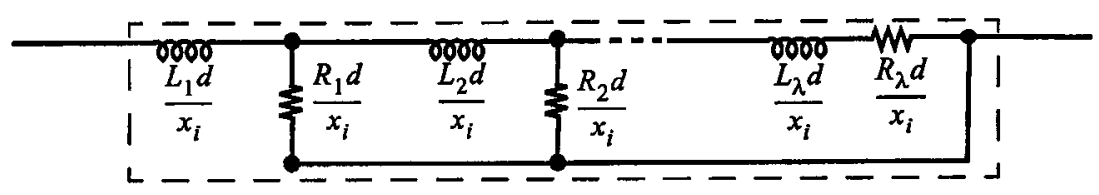

b) $Z_{\alpha i}$ circuit ( $\mathrm{RL}$ canonical configuration)

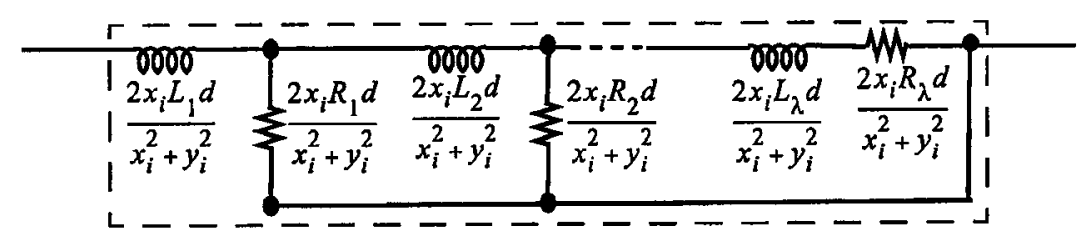

c) $Z_{\beta i}$ circuit (RL canonical configuration)
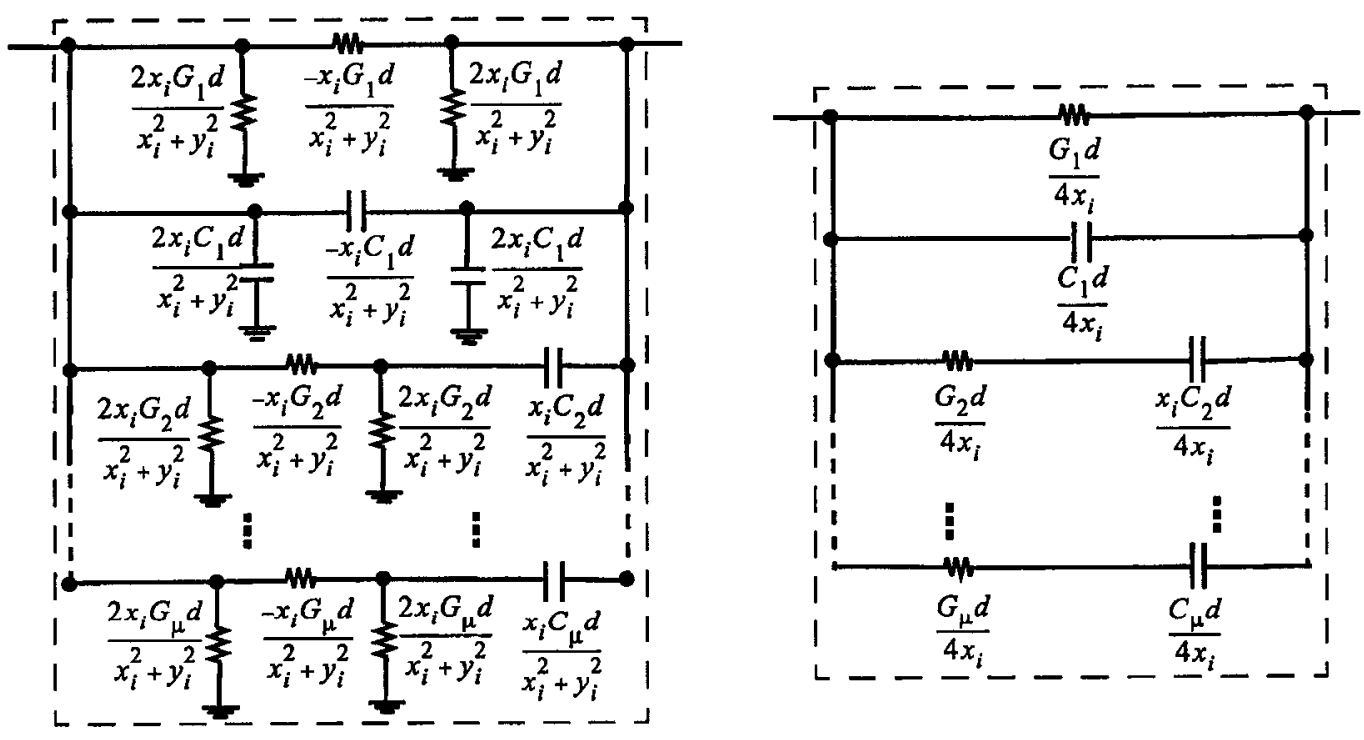

d) $Y_{\alpha i}$ circuit (RC Foster configuration)

e) $Y_{\beta i}$ circuit (RC Foster configuration)

Figure 5.8 Realization of two-conductor transmission-line with frequency-dependent parameters for complex pole-zero subsection of (5.5) 


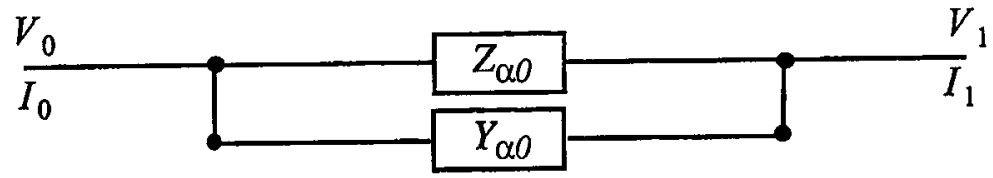

a) Real pole-zero subsection

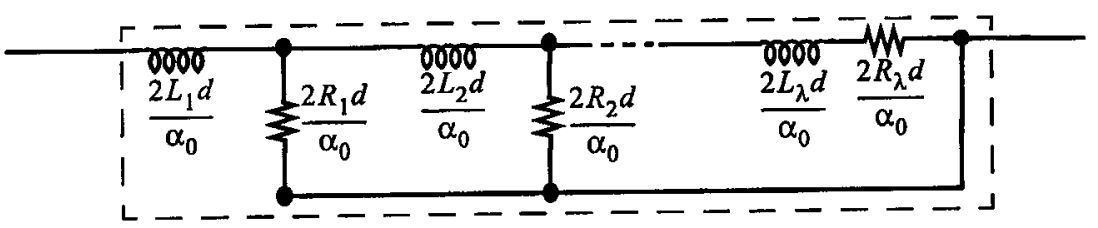

b) $Z_{\alpha 0}$ circuit (RL canonical configuration)

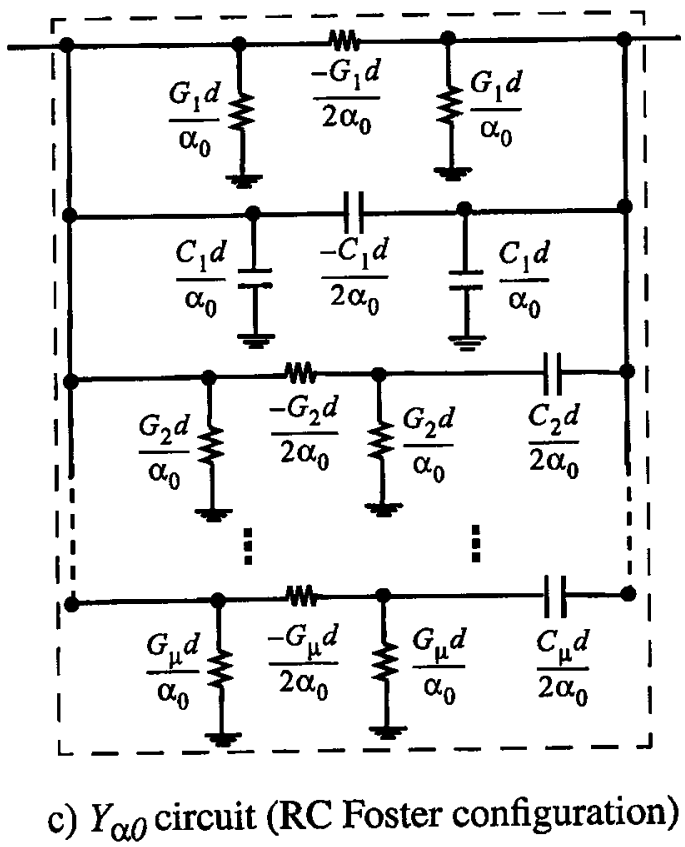

Figure 5.9 Realization of two-conductor transmission-line with frequency-dependent parameters for the real pole-zero subsection of (5.6) 


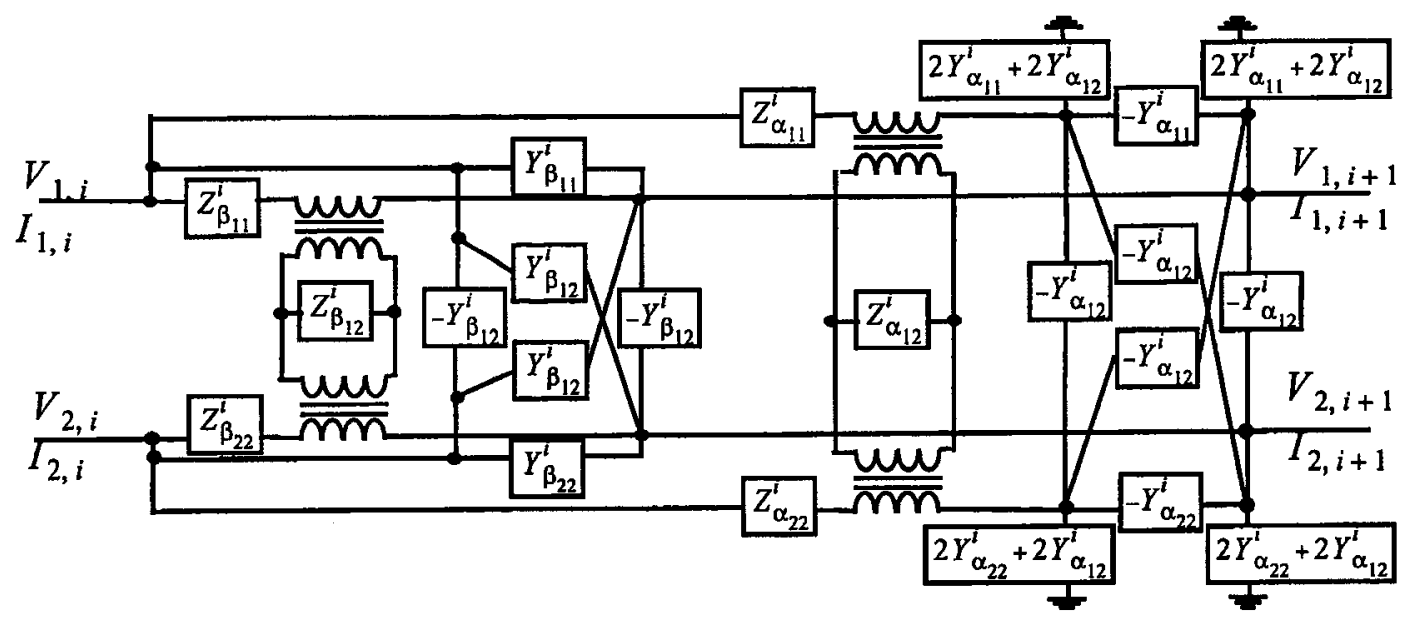

a) Complex pole-zero subsection
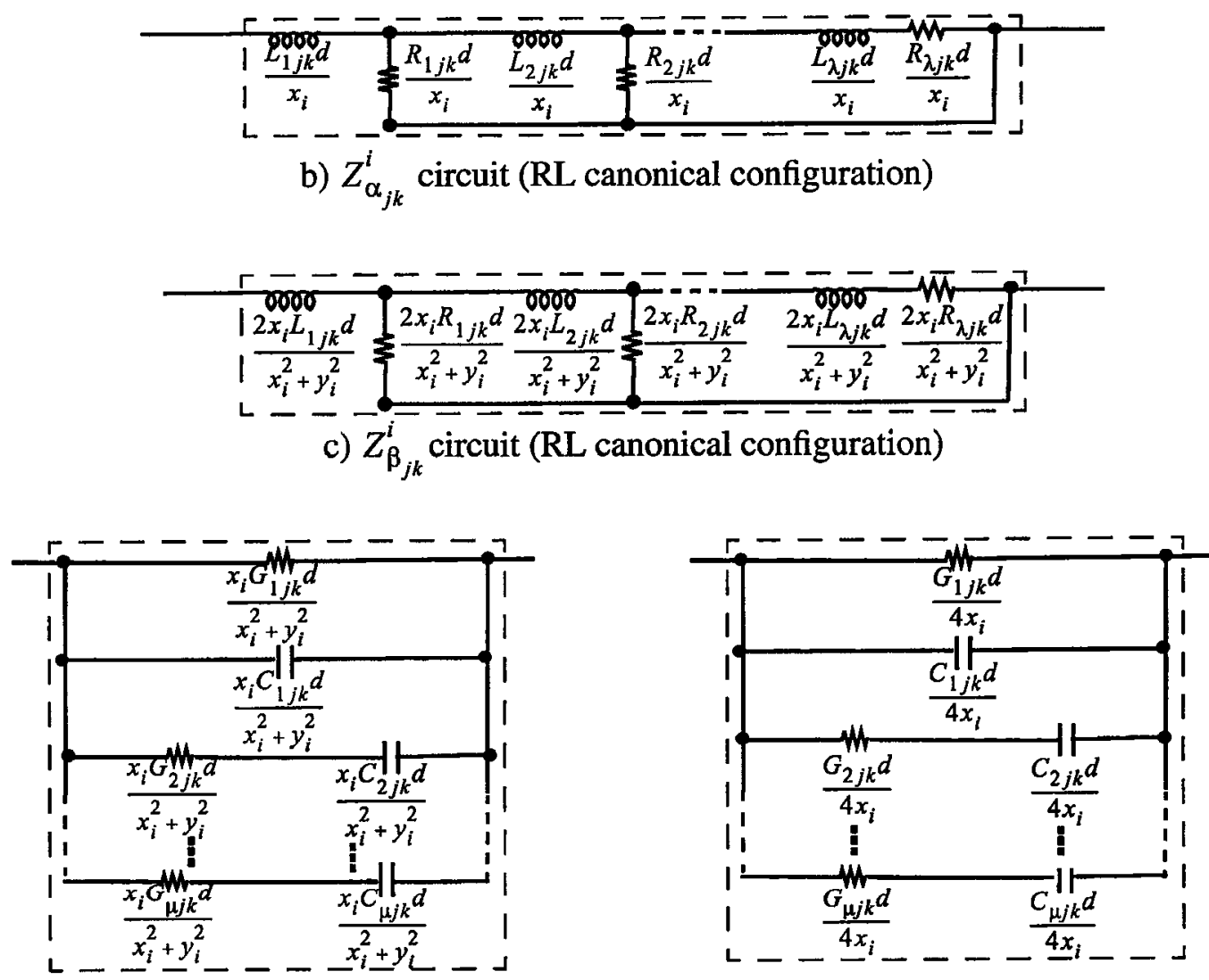

d) $Y_{\alpha_{j k}}^{i}$ circuit (RC Foster configuration)

e) $Y_{\beta_{j k}}^{i}$ circuit (RC Foster configuration)

Figure 5.10 Realization of MTL with frequency-dependent parameters for complex pole-zero subsection of (5.5) 


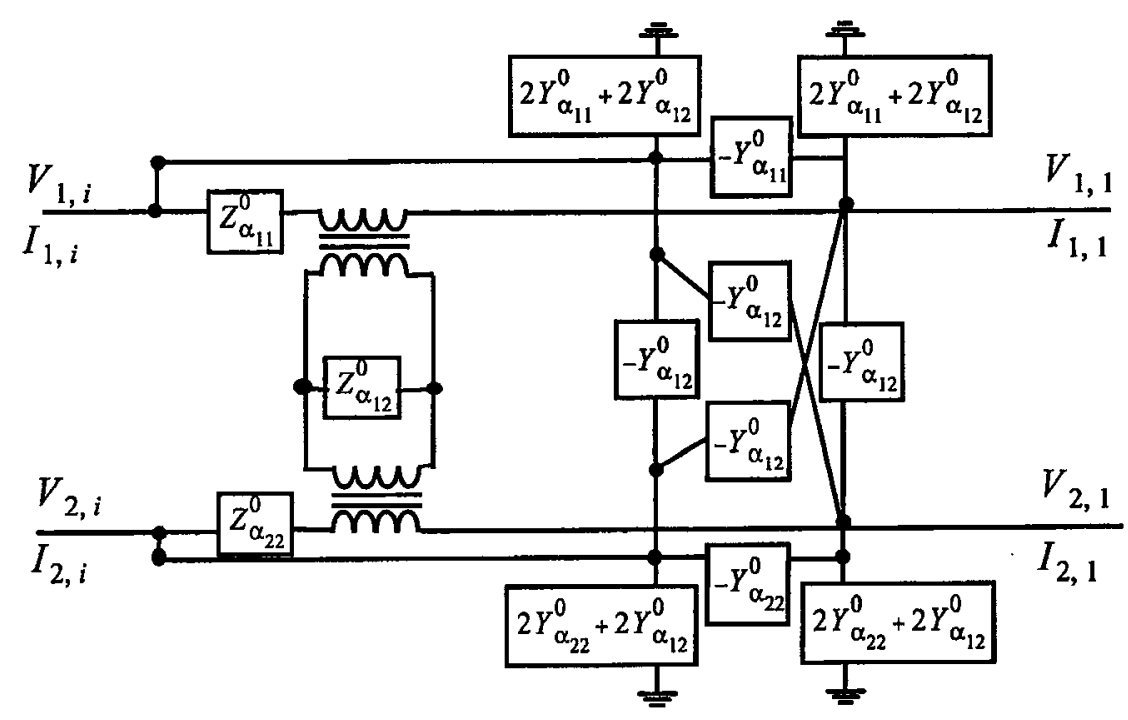

a) Real pole-zero subsection

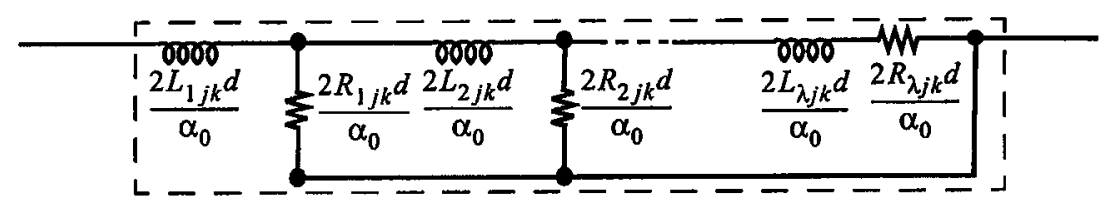

b) $Z_{\alpha_{j k}}^{0}$ circuit (RL canonical configuration)

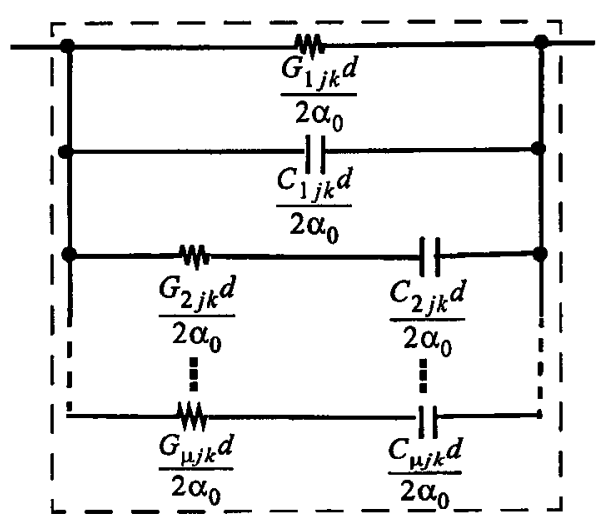

c) $Y_{\alpha_{j k}}^{0}$ circuit (RC Foster configuration)

Figure 5.11 Realization of MTL with frequency-dependent parameters for real pole-zero subsection of (5.6) 


\subsection{Numerical Examples}

Two examples of transmission line networks with frequency-dependent p.u.l. parameters are presented in this section to show the validity of the proposed method. The simulation results are obtained using HSPICE [93] and circuit equivalent realization of macromodels on a SUN Blade-1000 workstation with $900 \mathrm{MHz}$ UltraSPARC-III CPU.

\subsubsection{Example 5.1}

In this example, a transmission line network with nonlinear terminations (CMOS inverters) is shown in Figure 5.12. The p.u.l. parameters of each transmission line are listed in Table 5.1. The frequency-dependent data are modeled using $3 \mathrm{RL}$ sections for $R_{11}(s)+s L_{11}(s)$ and $3 \mathrm{RL}$ sections for $R_{12}(s)+\mathrm{s} L_{12}(s)$. The rational fitting for $R_{11}, L_{11}, R_{12}$ and $L_{12}$ are compared with the measured data values in Figure 5.13. The output transient response corresponding to an input voltage of a trapezoidal pulse of amplitude $5 \mathrm{~V}$ with a rise/fall time of $0.1 \mathrm{~ns}$, pulse width of $5 \mathrm{~ns}$ and a period of $10 \mathrm{~ns}$ in shown in Figure 5.14. Both the MRA macromodel (order 8) and the conventional lumped model (48 sections) give similar results, however, the proposed method requires $83 \%$ fewer variables to model each transmission line network. In terms of CPU time, the proposed method required 11 seconds to simulate the entire nonlinear network while the conventional lumped model required 133 seconds. 


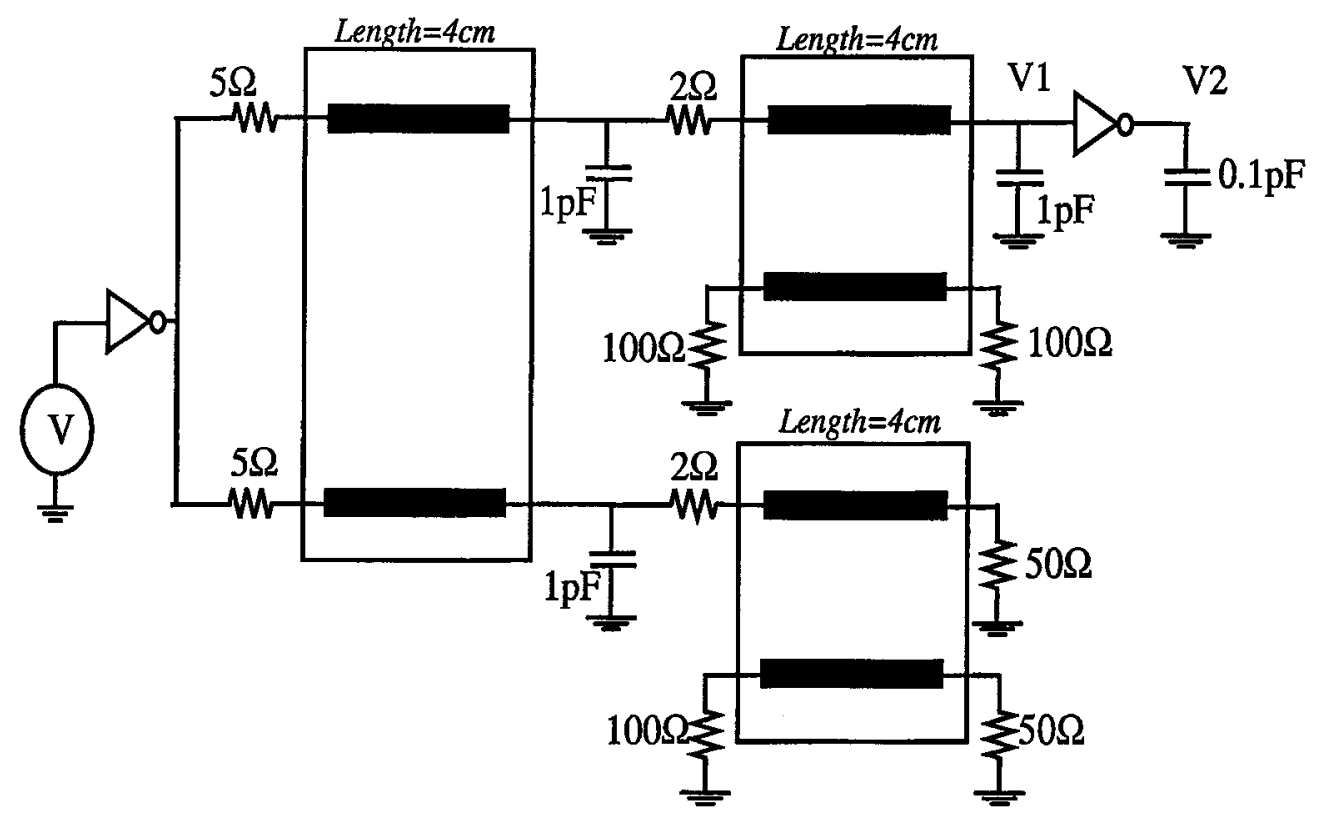

Figure 5.12 Nonlinear circuit with frequency-dependent parameters (Example 5.1)

\begin{tabular}{|c|c|c|c|c|}
\hline Freq(GHz) & $R_{11}(\Omega / m)$ & $R_{12}(\Omega / \mathrm{m})$ & $L_{11}(n H / m)$ & $L_{12}(\mathrm{nH} / \mathrm{m})$ \\
\hline 0 & 4.63 & 0.44 & 337 & 58.4 \\
\hline $1.1 \mathrm{e}-2$ & 6.47 & 0.44 & 230 & 17.2 \\
\hline 0.12 & 15.6 & 1.48 & 209 & 15.0 \\
\hline 0.38 & 28.1 & 2.72 & $\overline{203}$ & 14.4 \\
\hline 0.76 & 39.9 & 3.88 & 200 & 14.1 \\
\hline 1.21 & 50.5 & 4.90 & 199 & 13.9 \\
\hline 1.94 & 63.8 & 6.18 & 197 & 13.8 \\
\hline 2.45 & 71.7 & 6.94 & 197 & 13.7 \\
\hline 3.10 & 80.6 & 7.80 & 196 & 13.7 \\
\hline$\overline{3.91}$ & 90.6 & 8.75 & 195 & 13.6 \\
\hline 4.95 & 102 & 9.83 & 195 & 13.6 \\
\hline 6.26 & 115 & 11.0 & 195 & 13.6 \\
\hline$R_{12}=$ & $L_{12}$ & & $\begin{array}{cc}193 & -1 \\
-1.53 & 1\end{array}$ & $/ m$ \\
\hline
\end{tabular}

Table 5.1 P.u.l. parameters (Example 5.1) 


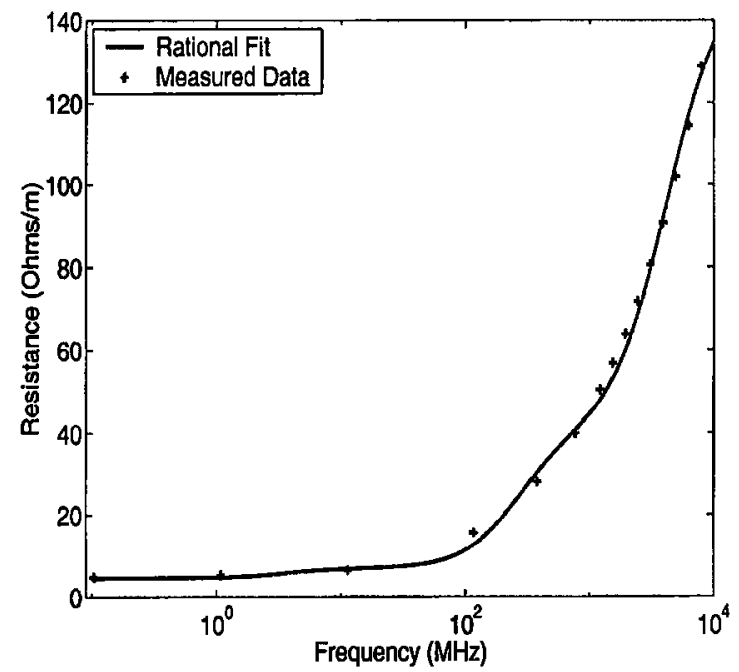

a) $R_{1 I}$ versus frequency

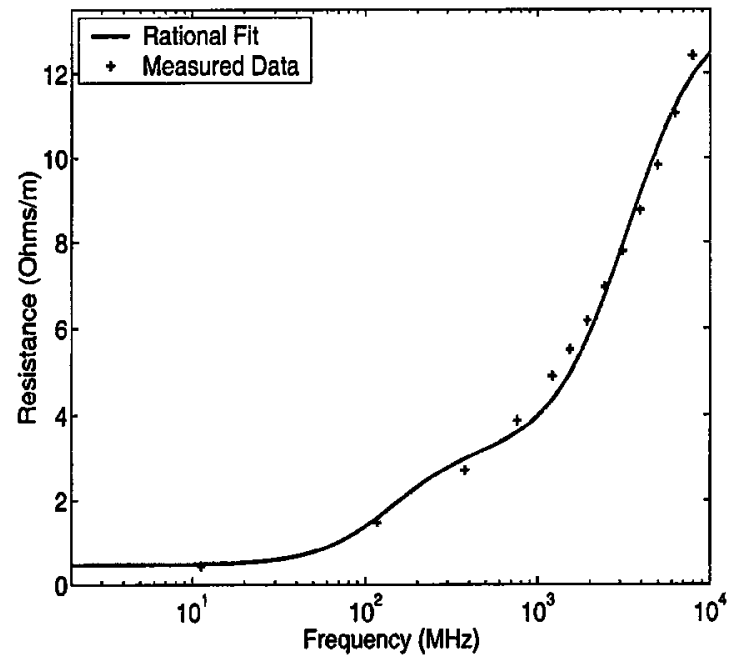

c) $R_{12}$ versus frequency

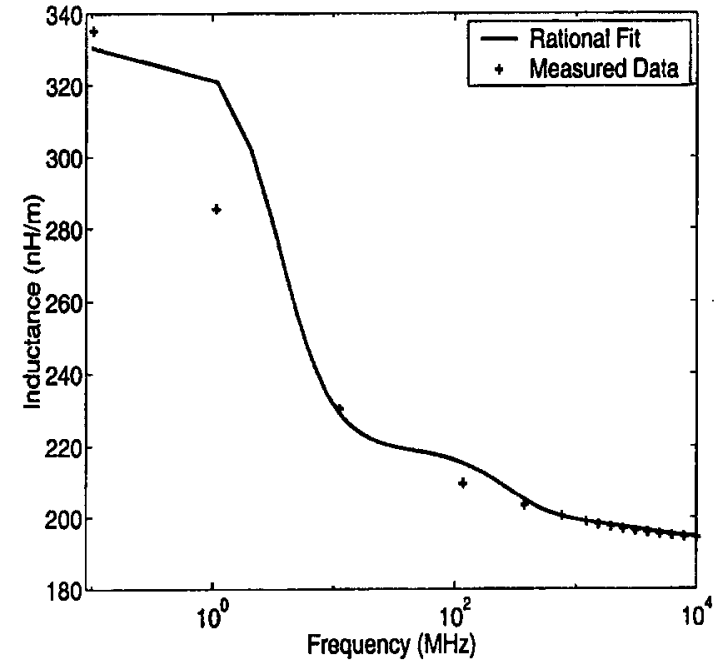

b) $L_{11}$ versus frequency

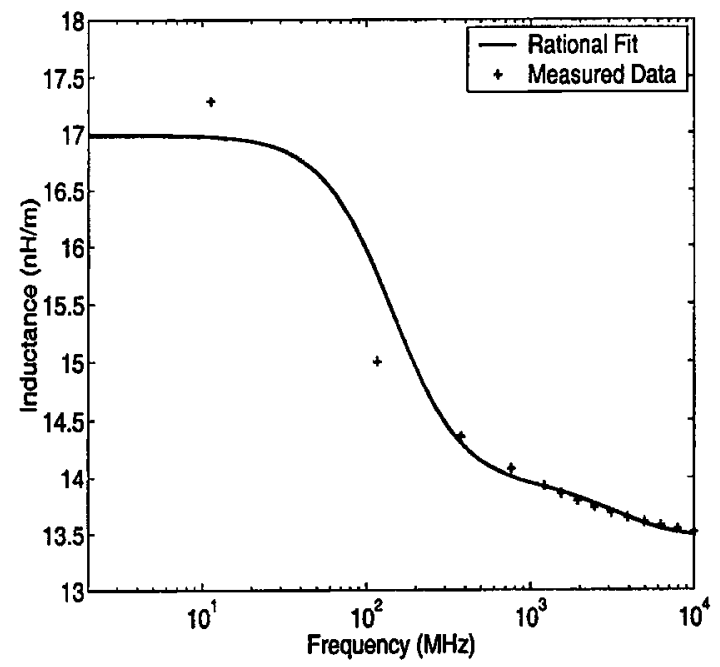

d) $L_{12}$ versus frequency

Figure 5.13 Fitting of the p.u.l. parameters (Example 5.1) 


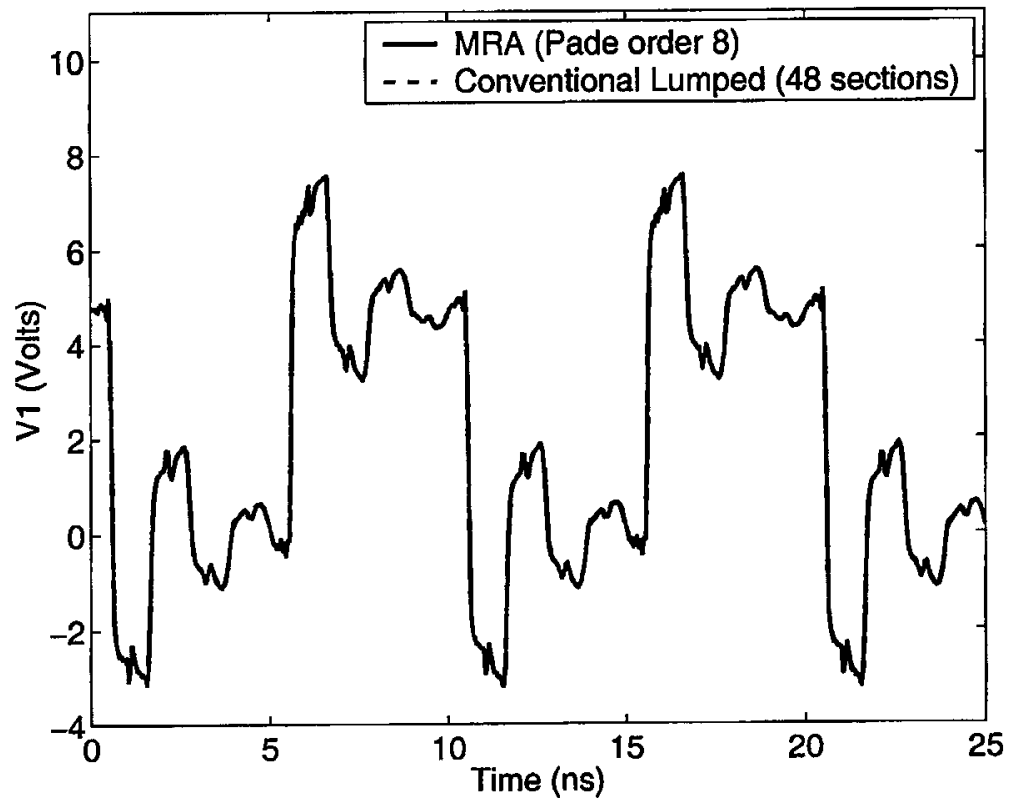

a) At node V1

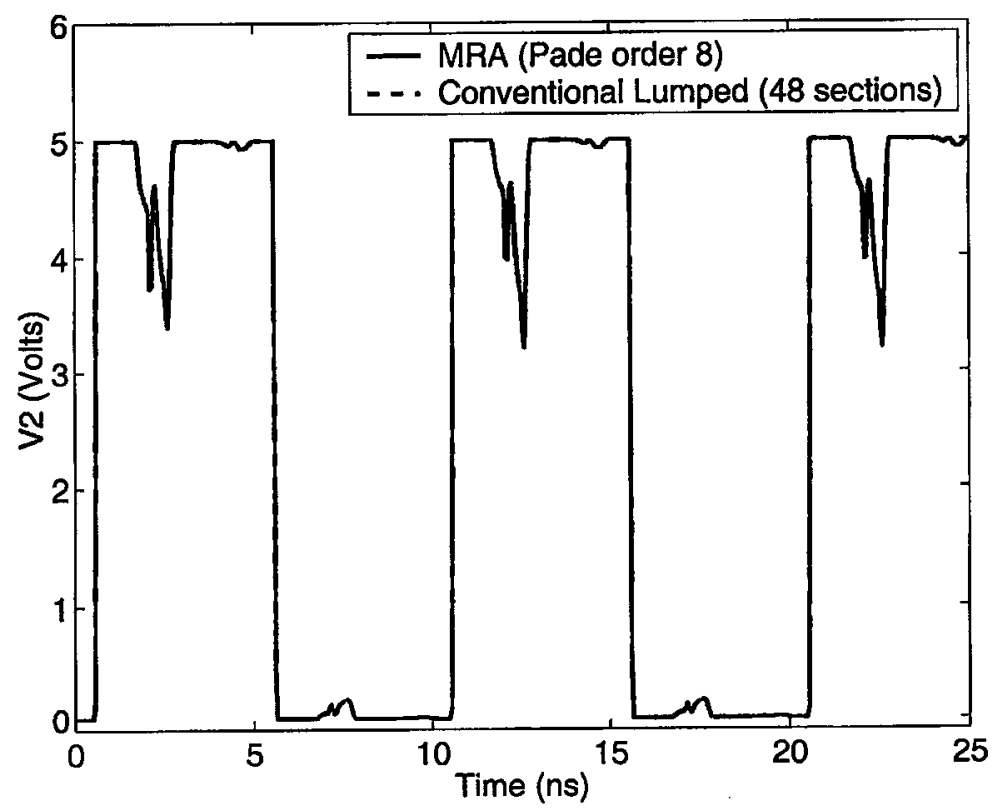

b) At node V2

Figure 5.14 Transient response of nonlinear circuit (Example 5.1) 


\subsubsection{Example 5.2}

The seven-transmission line network of Figure 4.17 (example 4.3) is considered. The p.u.l. parameters of each transmission line are frequency-dependent and are listed in Table 5.2. The transmission line parameters are modeled using $3 \mathrm{RL}$ sections for the $R(s)+s L(s)$ and $4 \mathrm{RC}$ sections for $G(s)+s C(s)$. The rational fittings of the p.u.l. parameters for are compared with the measured data values in Figure 5.15. The output transient responses (nodes $P 2$ and Vout) corresponding to a trapezoidal pulse of amplitude $5 \mathrm{~V}$ with a rise/fall time of $0.1 \mathrm{~ns}$, pulse width of $5 \mathrm{~ns}$ and a period of $10 \mathrm{~ns}$ are shown in Figure 5.16. Both the MRA macromodel (order 4) and the conventional lumped model (28 sections) give similar results, however, the proposed method requires $86 \%$ fewer variables to model each transmission line network. In terms of CPU time, the proposed method required 10 seconds to simulate the entire nonlinear network while the conventional lumped model required 74 seconds.

\begin{tabular}{c|c|c|c|c}
\hline Freq $(\mathrm{GHz})$ & $R(\Omega / m)$ & $L(n H / m)$ & $G(\mathrm{~S} / \mathrm{m})$ & $C(p F / m)$ \\
\hline 0 & 2.35 & 469.4 & 0 & 142.1 \\
\hline $1.60 \mathrm{e}-3$ & 2.72 & 385.3 & $3.48 \mathrm{e}-6$ & 141.1 \\
\hline $1.15 \mathrm{e}-2$ & 3.28 & 369.9 & $2.51 \mathrm{e}-5$ & 140.6 \\
\hline 0.145 & 8.26 & 360.6 & $3.17 \mathrm{e}-4$ & 140.0 \\
\hline 0.596 & 16.6 & 357.4 & $1.30 \mathrm{e}-3$ & 139.7 \\
\hline 1.39 & 25.3 & 356.0 & $3.03 \mathrm{e}-3$ & 139.5 \\
\hline 1.84 & 29.2 & 355.7 & $4.01 \mathrm{e}-3$ & 139.5 \\
\hline 2.44 & 33.6 & 355.3 & $5.32 \mathrm{e}-3$ & 139.4 \\
\hline 3.23 & 38.7 & 355.0 & $7.05 \mathrm{e}-3$ & 139.4 \\
\hline 4.29 & 44.5 & 354.7 & $9.35 \mathrm{e}-3$ & 139.3 \\
\hline 5.68 & 51.3 & 354.5 & $1.24 \mathrm{e}-2$ & 139.2 \\
\hline
\end{tabular}

Table 5.2 P.u.l. parameters (Example 5.2) 


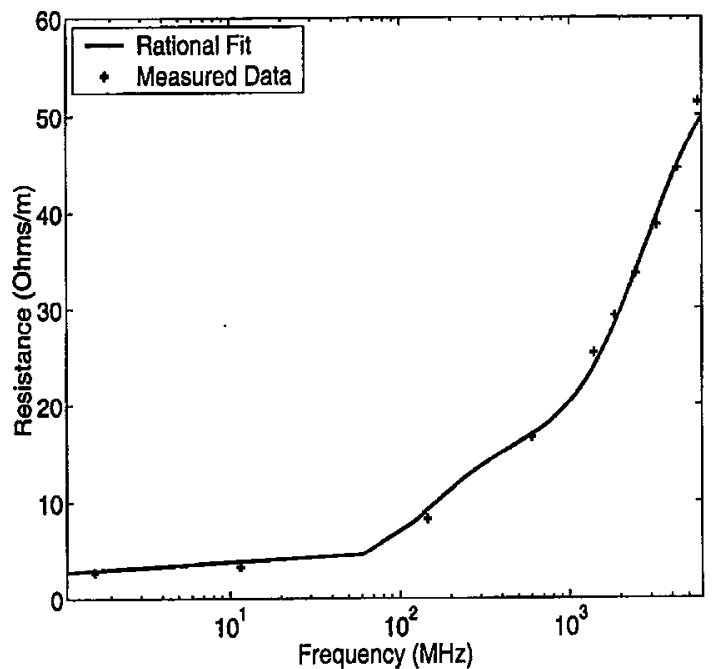

(a) Resistance versus frequency

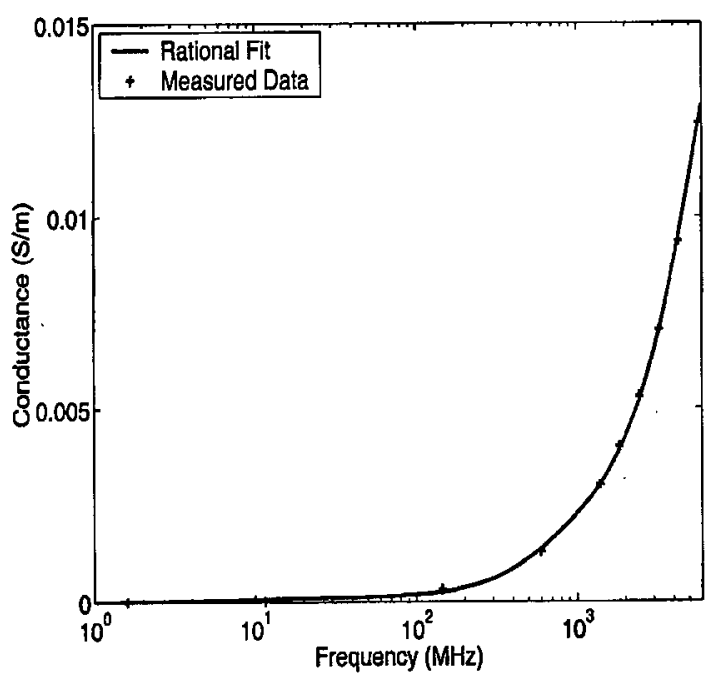

(c) Conductance versus frequency

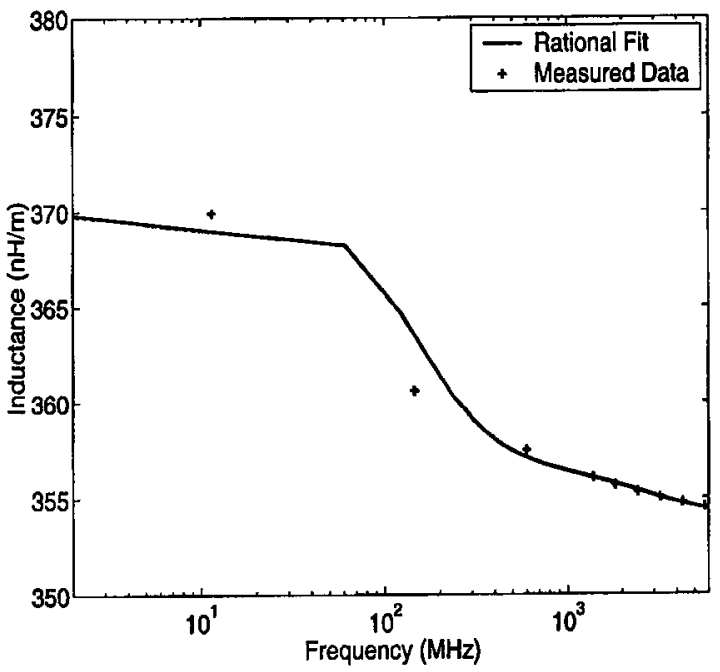

(b) Inductance versus frequency

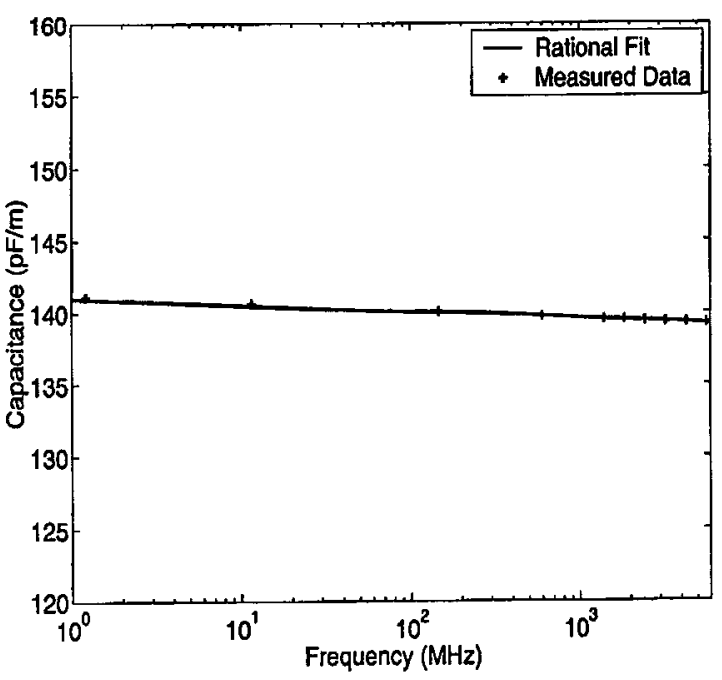

(d) Capacitance versus frequency

Figure 5.15 Fitting of the p.u.l. parameters (Example 5.2) 


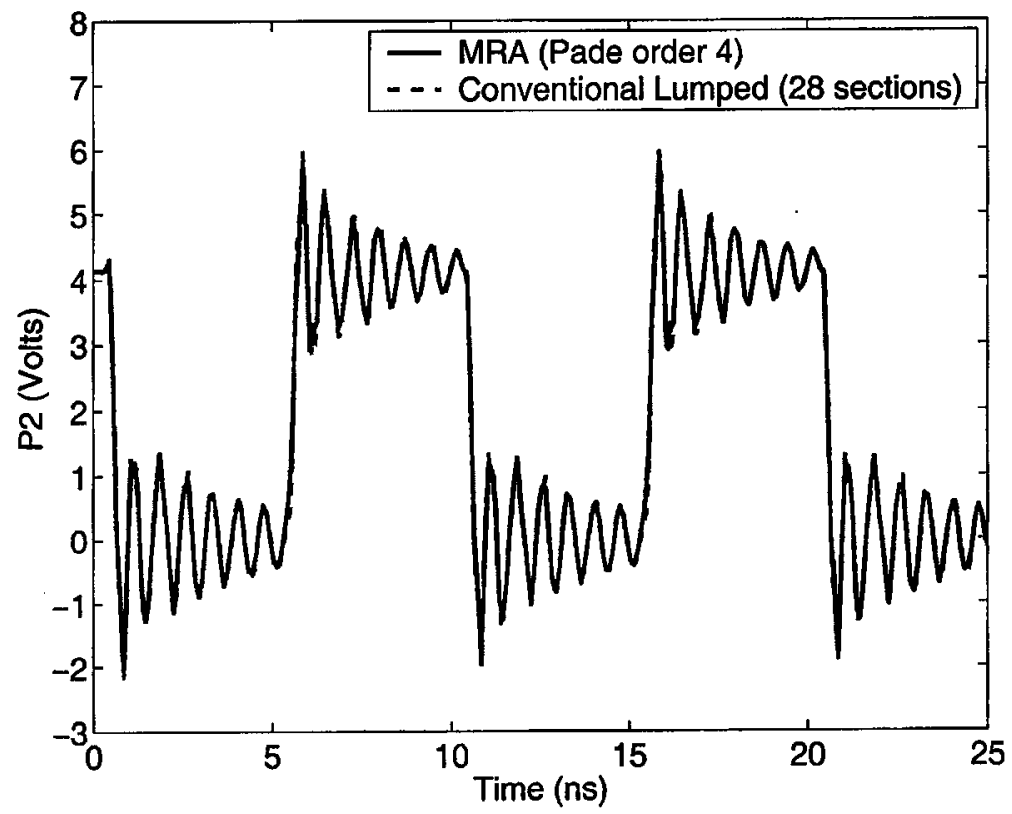

a) At node P2

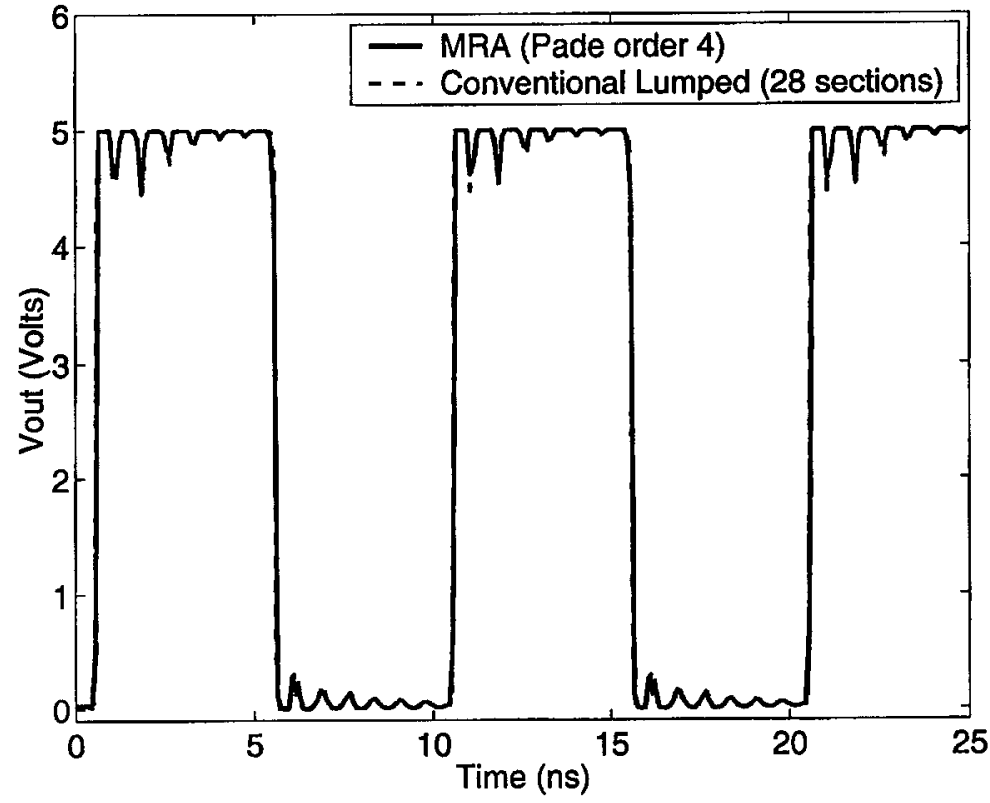

b) At node Vout

Figure 5.16 Transient response of nonlinear network (Example 5.2) 


\section{Chapter 6}

\section{Addressing Transient Errors in Passive Rational Macromodels}

\subsection{Introduction}

Rational macromodels are often used to model transmission lines [2]-[3]. However, for long delay transmission lines or weak signals such as transient responses of victim lines, rational macromodels can yield high-frequency transient errors. In order to address the problem of faulty transient response due to high-frequency errors, a new algorithm is presented. The proposed algorithm provides a mechanism to control the asymptotic behavior of high-frequency impulse response while matching the response up to the maximum frequency of interest. This leads to significant reduction in errors of transient responses. The concepts proposed in this chapter are applied to the matrix rational approximation (MRA) algorithm for passive macromodeling of transmission lines [62]-[63]. It is to be noted that, the principles stated in this chapter are general in nature and they can also be included in other rational function based macromodeling algorithms available in the literature, with appropriate modifications.

The organization of this chapter is as follows. The basic theory to minimize high frequency transient errors in rational macromodels of transmission lines is described in section 5.2. Section 5.3 applies these concepts to the MRA macromodel, while preserving passivity. The time-domain realization for the new MRA macromodel is provided in sec- 
tion 5.4. Numerical examples are presented in section 5.5 to demonstrate the validity of the proposed method.

\subsection{Concepts Pertinent to the Proposed Algorithm}

Broadly speaking, the algorithms used to model transmission lines can be classified into two categories. The first one includes techniques based on the generalized method of characteristics [21]-[25]. The second category is based on passive macromodeling of transmission lines [13], [32]-[33], [46], [49].

In general, method of characteristics extracts the line delay of the transfer function characterizing the frequency response of the transmission line. An important advantage of the method of characteristics approach is that, since it extracts the line delay explicitly, the corresponding transient responses generally do not exhibit spurious ripples in the earlytime region. However, the method of characteristics can be CPU expensive in modeling lossy transmission lines in the presence of nonlinear elements. In addition, it does not guarantee the passivity of macromodels. Passivity is an important property to satisfy because macromodels that are stable but not passive can produce unstable networks when connected to other stable, even passive loads. This can lead to faulty transient simulation.

On the other hand, passive macromodeling schemes [13], [32]-[33], [46], [49] guarantee the passivity of macromodels, and lead to macromodels in terms of ordinary differential equations. Most of these algorithms employ some kind of approximation in the frequency-domain to match the impulse response up to a maximum frequency of interest $\left(f_{\max }\right)$. However, the behavior after $f_{\max }$ is generally not considered, which can lead to significant errors in the impulse transient response, especially in the early-time period (spurious ripples) [62]-[63], [66]. This can affect the accuracy of the transient response at all other time-points when the macromodel is included during the simulation of a large network. Also, the above problem can be aggravated in the presence of sharp rise times or with smaller capacitive loads. To minimize these transient errors, the order of the approximation required would be very high, making the macromodel inefficient. 


\subsubsection{Concept}

For the early time response to be reasonably flat (corresponding to the flat delay portion of the transmission line response), it is desirable to have (as many as possible) initial derivatives of the impulse time response to be equal to zero. Note that the early-time impulse response is mainly influenced by the following relationship:

$$
h\left(0^{+}\right)=\lim _{s \rightarrow \infty} s H_{m n}(s)
$$

where ' $s$ ' is the Laplace operator, $H_{m n}(s)$ represents the frequency-domain rational function, $m$ is the order of numerator polynomial, $n$ is the order of denominator polynomial, and $h\left(0^{+}\right)$represents the early-time response (around $t=0$ ). Next, assuming that $h\left(0^{+}\right)=0$, then the first derivative of the impulse response becomes

$$
h^{(1)}\left(0^{+}\right)=\lim _{s \rightarrow \infty} s^{2} H_{m n}(s)
$$

Similarly, if the first $p$ derivatives are zero, then the $h^{(p+1)}\left(0^{+}\right)$can be expressed as

$$
h^{(p+1)}\left(0^{+}\right)=\lim _{s \rightarrow \infty} s^{p+2} H_{m n}(s)
$$

Observing (6.1)-(6.3) one can note that, to obtain flat response around $t=0$ (i.e., to have as many as possible initial derivatives of the impulse time response to be equal to zero), the transfer-admittances represented by $H_{m n}(s)$ must satisfy $n>m$. This can be further illustrated using a numerical example as follows: for example, let the transfer-admittance $Y_{12}$ of a transmission line be given by

$$
Y_{12}=\frac{1}{s^{4}+7 s^{3}+17 s^{2}+17 s+6}
$$

then $y_{12}\left(0^{+}\right)=0, y_{12}^{(1)}\left(0^{+}\right)=0$ and $y_{12}^{(2)}\left(0^{+}\right)=0$. Since the first few time derivatives of (6.4) are zero, the delay of the transmission line found in this case is reasonably approximated as a flat response. However, if the order of the numerator is much less than the order of denominator, then the rational approximation has less degree of freedom to capture the frequency response. A practical compromise is to have the numerator be few or- 
ders less than the denominator. This enables the transfer-admittance to have enough degrees of freedom, while ensuring that the first few derivatives are zero.

\subsubsection{Limitations of Current Passive Macromodeling Algorithms}

Rational macromodeling algorithms such as the MRA macromodel, efficiently capture the frequency response between ( 0 and $f_{\max }$ ). However, these macromodels can lead to significant high-frequency errors, causing faulty early time response. The reason for these errors can be explained by noting the final rational forms of individual entries in the admittance matrix. For example, the Y-parameters of the MRA macromodel are described by (3.12). For the sake of simplicity and without loss of generality, assume that the perunit-length (p.u.1.) parameters are scalars. For order $N($ denoted as $O(N))$, the orders of passive rational-form of the Y-parameters of (3.12) are given by

$$
\begin{aligned}
& {\left[\begin{array}{ll}
\boldsymbol{Y}_{11} & \boldsymbol{Y}_{12} \\
\boldsymbol{Y}_{21} & \boldsymbol{Y}_{22}
\end{array}\right] \Rightarrow\left[\begin{array}{ll}
\frac{O(N)}{O(N-1)} & \frac{O(N)}{O(N-1)} \\
\frac{O(N)}{O(N-1)} & \frac{O(N)}{O(N-1)}
\end{array}\right]+\left[\begin{array}{ll}
\frac{O(N-1)}{O(N)} & \frac{O(N-1)}{O(N)} \\
\frac{O(N-1)}{O(N)} & \frac{O(N-1)}{O(N)}
\end{array}\right] } \\
& \Rightarrow \frac{1}{O(2 N-1)}\left\{\left[\begin{array}{ll}
O(2 N) & O(2 N) \\
O(2 N) & O(2 N)
\end{array}\right]+\left[\begin{array}{ll}
O(2 N-2) & O(2 N-2) \\
O(2 N-2) & O(2 N-2)
\end{array}\right]\right\} \\
& \Rightarrow\left[\begin{array}{ll}
\frac{O(2 N)}{O(2 N-1)} & \frac{O(2 N)}{O(2 N-1)} \\
\frac{O(2 N)}{O(2 N-1)} & \frac{O(2 N)}{O(2 N-1)}
\end{array}\right]
\end{aligned}
$$

As seen, the transfer admittances such as $Y_{12}$, have the rational form with orders $O(2 N) /$ $O(2 N-1)$. Such a form mainly arises due to theorem 3.1 (Chapter 3 ) which strictly requires the rational form of $(N) /(N)$ for the approximation of exponential matrix. The above conclusion is true even for other types of passive macromodeling techniques in the literature [13], [32]-[33], where the order difference between the denominator and numerator (i.e. $n$ $m$ ) ranges between negative one to one. Obviously, transfer admittances of this form do 
not satisfy the conditions set by (6.1)-(6.3) (i.e., initial few derivatives of these transfer admittances are not equal to zero). Hence, these macromodels are susceptible to significant errors in the impulse transient response, especially in the early time region.

A further investigation of the rational-form of Y-parameters of (3.12) tells us why it is difficult to control the high-frequency response such that it satisfies the conditions required by (6.1)-(6.3) in order to produce a reasonably flat delay. For example, consider the case when $N$ is even (without loss of generality) for a two-conductor transmission line system. The corresponding rational function of the Y-parameters of (3.12) can be re-arranged as

$$
\begin{aligned}
& \left.\begin{array}{l}
Y_{11} \\
Y_{22}
\end{array}\right\}=\frac{Q_{N_{11}} Q_{N_{22}}+Q_{N_{12}} Q_{N_{21}}}{2 Q_{N_{12}} Q_{N_{22}}}=\frac{\mu_{N}(a b)^{N}+\sum_{i=0}^{N-1}\left(\mu_{i}+\rho_{i}\right)(a b)^{i}}{a\left(\sum_{i=0}^{N-1} \varphi_{i}(a b)^{i}\right)} \\
& \left.\begin{array}{l}
Y_{12} \\
Y_{21}
\end{array}\right\}=\frac{-Q_{N_{11}} Q_{N_{22}}+Q_{N_{12}} Q_{N_{21}}}{2 Q_{N_{12}} Q_{N_{22}}}=\frac{-\mu_{N}(a b)^{N}+\sum_{i=0}^{N-1}\left(-\mu_{i}+\rho_{i}\right)(a b)^{i}}{a\left(\sum_{i=0}^{N-1} \varphi_{i}(a b)^{i}\right)}
\end{aligned}
$$

where $Q_{N_{11}}, Q_{N_{12}}, Q_{N_{21}}$ and $Q_{N_{22}}$ are described by (3.11) and the predetermined coefficients $\mu_{i}, \rho_{i}$ and $\varphi_{i}$ in (6.6) are obtained by the scalar approximation of (3.7) using the following relations:

$$
\begin{gathered}
E v\left(Q_{N}(x)\right) E v\left(Q_{N}(x)\right)=\sum_{i=0}^{N} \mu_{i} x^{2 i} \\
\operatorname{Odd}\left(Q_{N}(x)\right) \operatorname{Odd}\left(Q_{N}(x)\right)=\sum_{i=0}^{N} \rho_{i} x^{2 i} \\
2 \operatorname{Odd}\left(Q_{N}(x)\right) E v\left(Q_{N}(x)\right)=\sum_{i=0}^{N-1} \varphi_{i} x^{(2 i+1)}
\end{gathered}
$$

where $E v(~)$ and $\operatorname{Odd}($ ) represent the even and odd functions, respectively. Noticing 
(6.6), it can be inferred that, by appropriately choosing the values for $\rho_{i}$ and $\mu_{i}$ such that $\mu_{i}=\rho_{i}$, (for example $\mu_{N-1}=\rho_{N-1}$ ), the transfer-admittances of (6.6) could be forced to have fewer polynomial terms on the numerator. However, since (6.6) does not have a $\rho_{N}$ coefficient, it is very difficult to remove the highest order polynomial term. This implies that, it is not possible to reduce the order of the numerator less than $N$, without violating passivity conditions. In order to address these difficulties we present a new algorithm in the next section, which also provides a mechanism to control the asymptotic behavior of the high-frequency impulse response of the transfer-admittance.

\subsection{Development of the Proposed Macromodeling Algorithm}

The objective of the proposed algorithm is to provide a mechanism to control the macromodel impulse response beyond $f_{\max }$ so as to minimize early-time ripples while preserving the accuracy and passivity of the macromodel. For this purpose, the proposed algorithm uses two different orders of approximation of the scalar exponential in (3.7), $(N) /(N)$ and $(N+1) /(N+1)$ that satisfy Theorem 3.1. Consider a particular order $N$, if $N$ is even (without loss of generality), then the approximation given by (3.7) can be expressed as:

$$
e^{x} \approx \frac{Q(x)}{Q(-x)}=\frac{\sum_{i=0}^{N} q_{i} x^{i}}{\sum_{i=0}^{N} q_{i}(-x)^{i}}
$$

Similarly, the other approximation corresponding to $N+1$ order can be denoted as

$$
e^{x} \approx \frac{\tilde{Q}(x)}{\tilde{Q}(-x)}=\frac{\sum_{i=0}^{N+1} \tilde{q}_{i} x^{i}}{\sum_{i=0}^{N+1} \tilde{q}_{i}(-x)^{i}}
$$

The MRA macromodel presented in chapter 3, is expressed in terms of $2 \times 2$ block matrices 
corresponding to the terminal voltages and currents (3.9), as

$$
\left[\begin{array}{ll}
\boldsymbol{Q}_{N_{11}} & \boldsymbol{Q}_{N_{12}} \\
\boldsymbol{Q}_{N_{21}} & \boldsymbol{Q}_{N_{22}}
\end{array}\right]\left[\begin{array}{c}
\boldsymbol{V}(d, s) \\
-\boldsymbol{I}(d, s)
\end{array}\right] \approx\left[\begin{array}{cc}
\boldsymbol{Q}_{N_{11}} & -\boldsymbol{Q}_{N_{12}} \\
-\boldsymbol{Q}_{N_{21}} & \boldsymbol{Q}_{N_{22}}
\end{array}\right]\left[\begin{array}{c}
\boldsymbol{V}(0, s) \\
I(0, s)
\end{array}\right]
$$

Using (6.8) and (6.9), the rational approximation can be expressed as

$$
\begin{aligned}
& {\left[\begin{array}{ll}
Q_{11} & Q_{12}
\end{array}\right]\left[\begin{array}{c}
V(d, s) \\
-I(d, s)
\end{array}\right] \approx\left[\begin{array}{ll}
Q_{11} & \boldsymbol{Q}_{12}
\end{array}\right]\left[\begin{array}{l}
V(0, s) \\
I(0, s)
\end{array}\right]} \\
& {\left[\begin{array}{ll}
\tilde{Q}_{21} & \tilde{Q}_{22}
\end{array}\right]\left[\begin{array}{c}
V(d, s) \\
-I(d, s)
\end{array}\right] \approx\left[\begin{array}{ll}
-\tilde{Q}_{21} & \tilde{Q}_{22}
\end{array}\right]\left[\begin{array}{l}
V(0, s) \\
I(0, s)
\end{array}\right]}
\end{aligned}
$$

where the coefficients of (6.8) are used to approximate (6.11) and the coefficients of (6.9) are used to approximate (6.12). Note that equations (6.11) and (6.12) are self-consistent and do not violate the passivity conditions set by Theorem 3.1. The polynomial matrices $\boldsymbol{Q}_{11}, \boldsymbol{Q}_{12}, \tilde{\boldsymbol{Q}}_{21}$ and $\tilde{\boldsymbol{Q}}_{22}$ are now defined as:

$$
\begin{aligned}
\boldsymbol{Q}_{11}=\sum_{i=0}^{N} q_{i}\left[\frac{1}{2}\left(1+(-1)^{i}\right)(\boldsymbol{a b})^{i / 2}\right] ; & \boldsymbol{Q}_{12}=\sum_{i=0}^{N} q_{i}\left[\frac{1}{2}\left(1-(-1)^{i}\right)(\boldsymbol{a b})^{\frac{i-1}{2}} a\right] \\
\tilde{\boldsymbol{Q}}_{21}=\sum_{i=0}^{N+1} \tilde{q}_{i}\left[\frac{1}{2}\left(1-(-1)^{i}\right)(\boldsymbol{b a})^{\frac{i-1}{2}} \boldsymbol{b}\right] ; & \tilde{\boldsymbol{Q}}_{22}=\sum_{i=0}^{N+1} \tilde{q}_{i}\left[\frac{1}{2}\left(1+(-1)^{i}\right)(\boldsymbol{b a})^{i / 2}\right]
\end{aligned}
$$

where $\boldsymbol{a}=(\boldsymbol{R}(s)+s \boldsymbol{L}(s)) d$ and $\boldsymbol{b}=(\boldsymbol{G}(s)+s \boldsymbol{C}(s)) d$. Subsequently, the form of the resulting Yparameters of (6.11) and (6.12) can be re-written as

$$
\begin{gathered}
{\left[\begin{array}{ll}
\boldsymbol{Y}_{11} & \boldsymbol{Y}_{12} \\
\boldsymbol{Y}_{21} & \boldsymbol{Y}_{22}
\end{array}\right]=\Psi_{1}+\Psi_{2}=\left[\begin{array}{cc}
\boldsymbol{H}_{w} & -\boldsymbol{H}_{w} \\
-\boldsymbol{H}_{w} & \boldsymbol{H}_{w}
\end{array}\right]+\left[\begin{array}{cc}
\tilde{\boldsymbol{H}}_{x} & \tilde{\boldsymbol{H}}_{x} \\
\tilde{\boldsymbol{H}}_{x} & \tilde{\boldsymbol{H}}_{x}
\end{array}\right]} \\
\boldsymbol{H}_{w}=\frac{1}{2}\left[\boldsymbol{Q}_{12}\right]^{-1} \boldsymbol{Q}_{11} ; \quad \tilde{\boldsymbol{H}}_{x}=\frac{1}{2}\left[\tilde{\boldsymbol{Q}}_{22}\right]^{-1} \tilde{\boldsymbol{Q}}_{21}
\end{gathered}
$$




\subsubsection{Two-Conductor Transmission Line Case}

Consider the case of two-conductor transmission line system. Using (6.14), the rational function of Y-parameters can be expressed as

$$
\begin{aligned}
& \left.\begin{array}{l}
Y_{11} \\
Y_{22}
\end{array}\right\}=\frac{Q_{11} \tilde{Q}_{22}+Q_{12} \tilde{Q}_{21}}{2 Q_{12} \tilde{Q}_{22}}=\frac{\sum_{i=0}^{N}\left(\mu_{i}+\rho_{i}\right)(a b)^{i}}{a\left(\sum_{i=0}^{N-1} \varphi_{i}(a b)^{i}\right)} \\
& \left.\begin{array}{l}
Y_{12} \\
Y_{21}
\end{array}\right\}=\frac{-Q_{11} \tilde{Q}_{22}+Q_{12} \tilde{Q}_{21}}{2 Q_{12} \tilde{Q}_{22}}=\frac{\sum_{i=0}^{N}\left(-\mu_{i}+\rho_{i}\right)(a b)^{i}}{a\left(\sum_{i=0}^{N-1} \varphi_{i}(a b)^{i}\right)}
\end{aligned}
$$

where the predetermined coefficients $\mu_{i}, \rho_{i}$ and $\varphi_{i}$ in (6.15) are obtained using the relations of (6.8) and (6.9)

$$
\begin{gathered}
E v(Q(x)) E v(\tilde{Q}(x))=\sum_{i=0}^{N} \mu_{i} x^{2 i} \\
\operatorname{Odd}(Q(x)) \operatorname{Odd}(\tilde{Q}(x))=\sum_{i=0}^{N} \rho_{i} x^{2 i} \\
2 \operatorname{Odd}(Q(x)) E v(\tilde{Q}(x))=\sum_{i=0}^{N-1} \varphi_{i} x^{(2 i+1)}
\end{gathered}
$$

$E v($ ) and $O d d($ ) correspond to the even and odd part of the polynomial, respectively.

\subsubsection{Controlling Asymptotic Behaviour of Impulse Response}

Asymptotic behavior of the high-frequency impulse response can be controlled using the following mechanism. In the formulation of (6.15), the order of the numerator of the transfer-admittance can be reduced by appropriately choosing the values for $\rho_{i}$ and $\mu_{i}$. For example, while computing the predetermined coefficients, imposing the constraint 


$$
\mu_{N}=\rho_{N}
$$

removes the highest order polynomial term of the transfer-admittance in (6.15) (i.e. denominator order - numerator order $=1$ in (6.1)). Similarly, imposing the constraints

$$
\mu_{N}=\rho_{N} ; \quad \mu_{N-1}=\rho_{N-1}
$$

leads to the removal of two highest order polynomial terms of the numerator of the transfer admittance in (6.15) (i.e. denominator order - numerator order $=3$ in (6.1)). This makes the transfer admittance to satisfy the conditions set by (6.1)-(6.3). In other words, if a total number of $k$ such constraints are used, then it ensures that the function and $2 k-3$ derivatives of the transfer-admittance are set to zero. For example, if $k=2$ then the order of transfer admittance $y_{12}$ given by $(6.15)$ is

$$
\left.\begin{array}{l}
Y_{12} \\
Y_{21}
\end{array}\right\}=\frac{\sum_{i=0}^{N-2}\left(-\mu_{i}+\rho_{i}\right)\left[(R+s L)(G+s C) d^{2}\right]^{i}}{(R+s L) d\left(\sum_{i=0}^{N-1} \varphi_{i}\left[(R+s L)(G+s C) d^{2}\right]^{i}\right)} \Rightarrow \frac{O(2 N-4)}{O(2 N-1)}
$$

In this case, using (6.1)-(6.3), we will have $y_{12}\left(0^{+}\right)=0$ and $y_{12}^{(1)}\left(0^{+}\right)=0$. It should be noted that if the number of constraints used in (6.18) are many and is comparable to the order of approximation then the macromodel has less degrees of freedom to capture the frequency response. To obtain accurate macromodels very few constraints should be used. For the examples provided in this chapter, only one or two constraints were used, which provided significant improvement in the time-domain response.

\subsubsection{Extension to Multi-conductor Transmission Lines}

Extension of the proposed algorithm to the case of multi-conductor transmission lines (MTLs) can be explained as follows. Consider the Y-parameter terms of (6.14) for the case of two-conductor transmission line. The functions $H_{w}$ and $\tilde{H}_{x}$ can be expressed in terms of continued fraction expansion as: 


$$
H_{w}=\kappa_{N} b+\frac{1}{\kappa_{N-1} a+\frac{1}{\kappa_{N-2} b+\ldots}} ;
$$

$$
\tilde{H}_{x}=\tau_{N+1} b+\frac{1}{\tau_{N} a+\frac{1}{\tau_{N-1} b+\ldots}}
$$

where $a=(R+s L) d, b=(G+s C) d$. The coefficients $\kappa$ 's and $\tau$ 's are the predetermined constants and are obtained using the continued-fraction representation of

$$
\begin{aligned}
& \frac{E v(Q)}{\operatorname{Ldd}(Q)}=\kappa_{N} x+\frac{1}{\kappa_{N-1} x+\frac{1}{\kappa_{N-2} x+\ldots}} \\
& \frac{\operatorname{Odd}(\tilde{Q})}{2 E v(\tilde{Q})}=\tau_{N+1} x+\frac{1}{\tau_{N-1} x+\frac{1}{\tau_{N-2} x+\ldots}} \\
& \because \ddots_{1} x
\end{aligned}
$$

where $Q$ and $\tilde{Q}$ are described by (6.8) and (6.9). Next, from (6.14) it can be noticed that the transfer-admittances are given by

$$
Y_{12}=Y_{21}=-H_{w}+\tilde{H}_{x}
$$

Hence, while computing the predetermined constants, if we impose the condition,

$$
\kappa_{N}=\tau_{N+1}
$$

then it leads to the cancellation of the highest order polynomial term of the numerator of the transfer admittance of (6.15) (note that the condition set by (6.24) is also equivalent to setting $\mu_{N}=\rho_{N}$ as described by (6.17)). Proceeding further, if we also make

$$
\kappa_{N}=\tau_{N+1} ; \quad \kappa_{N-1}=\tau_{N}
$$

then it leads to the cancellation of the two highest polynomial terms, since the constraints 
of (6.25) are equivalent to the ones set by (6.17) $\left(\mu_{N}=\rho_{N}\right.$ and $\left.\mu_{N-1}=\rho_{N-1}\right)$. If additional constraints of the type (6.25) are included during the computation of predetermined constants, then the order of the numerator of the transfer admittance can be reduced even further.

Using the similar approach discussed above, for the of MTLs, the matrices of (6.14) can be expressed as

$$
\begin{gathered}
\boldsymbol{H}_{w}=\kappa_{N} b+\left(\kappa_{N-1} a+\left(\ldots+\left(\kappa_{2} b+\left(\kappa_{1} a\right)^{-1}\right)^{-1}\right)^{-1}\right)^{-1} \\
\tilde{\boldsymbol{H}}_{x}=\tau_{N+1} b+\left(\tau_{N} a+\left(\ldots+\left(\tau_{2} a+\left(\tau_{1} b\right)^{-1}\right)^{-1}\right)^{-1}\right)^{-1}
\end{gathered}
$$

Next, imposing the constraints similar to (6.24) and (6.25) while computing the predetermined constants will help to control the asymptotic behaviour of the transfer admittance. Note the important advantage here, in the MTL admittance approximation given by (6.26), the predetermined coefficients $\kappa_{i}, \tau_{i}$ are obtained by the scalar approximation of (6.8) and (6.9).

\subsubsection{Computation of Predetermined Coefficients}

This section describes the formulation of the minimax objective function for predetermining the coefficients of (6.8) and (6.9). The minimax objective function can be obtained by expressing the $N^{t h}$ and $(N+1)^{t h}$ order approximations given by (6.8) and (6.9) in terms of product of second order factors as

$$
\begin{gathered}
e^{s} \approx \frac{Q(s)}{Q(-s)}=\frac{\prod_{i=1}\left(s^{2}+g_{1, i} s+g_{0, i}\right)}{\prod_{i=1}\left(s^{2}-g_{1, i} s+g_{0, i}\right)} \\
e^{s} \approx \frac{\tilde{Q}(s)}{\tilde{Q}(-s)}=\frac{\left(s+h_{0,0}\right) \prod_{i=1}\left(s^{2}+h_{1, i} s+h_{0, i}\right)}{\left(-s+h_{0,0}\right) \prod_{i=1}\left(s^{2}-h_{1, i} s+h_{0, i}\right)}
\end{gathered}
$$


Equations (6.27) and (6.28) satisfy the form of (3.7) of theorem 3.1, which is required for passivity. Imposing the constraints that $g_{0, i}, g_{1, i}, h_{0, i}, h_{1, i}>0$ for all values of $i$ ensures that the Hurwitz conditions of theorem 3.1 are satisfied. The goal is to minimize the error function for a given interval, such that

$$
\max _{\left[0, \omega_{\max }\right]}\left(W_{1}(\omega)\left|e^{j \omega}-\frac{Q(j \omega)}{Q(-j \omega)}\right|+W_{2}(\omega) \mid e^{j \omega}-\frac{\tilde{Q}(j \omega)}{\tilde{Q}(-j \omega)}\right) \quad \text { is } \quad \text { minimum }
$$

where $s=j \omega$. The variables $W_{1}(\omega)$ and $W_{2}(\omega)$ are the weight functions at the angular frequency $\omega$. Separating (6.29) in terms of real and imaginary parts, the minimax objective function can be expressed as

$$
\begin{aligned}
& \max \left(W_{r e^{\left(\omega_{k}\right)}}\left(\cos \omega_{k}-F_{1}\right)^{2}+W_{i m^{\left(\omega_{k}\right)}}\left(\sin \omega_{k}-F_{2}\right)^{2}+\right. \\
& \left.W_{r e^{\left(\omega_{k}\right)}}\left(\cos \omega_{k}-\tilde{F}_{1}\right)^{2}+W_{i m^{\left(\omega_{k}\right)}}\left(\sin \omega_{k}-\tilde{F}_{2}\right)^{2}\right) \text { is minimum }
\end{aligned}
$$

such that $g_{0, i}, g_{1, i}, h_{0, i}, h_{1, i}>0$ for all values of $i$. The variables $W_{r e^{\left(\omega_{k}\right)}}, W_{i m}{ }^{\left(\omega_{k}\right)}$ are the weight functions at the angular frequency $\omega_{k}$, where $\omega_{k}$ ranges from $0 \leq \omega_{1}<\ldots<\omega_{k} \leq \omega_{\max }$ and

$$
\begin{aligned}
& F_{1}=\operatorname{Re}\left(\frac{Q_{\left(\omega_{k}\right)}}{\left.Q_{\left(-\omega_{k}\right)}\right)}=\frac{E v\left(Q_{\left(\omega_{k}\right)}\right)^{2}+\operatorname{Odd}\left(Q_{\left.\left(\omega_{k}\right)\right)^{2}}\right.}{E v\left(Q_{\left(\omega_{k}\right)}\right)^{2}-\operatorname{Odd}\left(Q_{\left(\omega_{k}\right)}\right)^{2}}\right. \\
& F_{2}=\operatorname{Im}\left(\frac{Q_{\left(\omega_{k}\right)}}{Q_{\left(-\omega_{k}\right)}}\right)=\frac{2 E v\left(Q_{\left(\omega_{k}\right)}\right) \operatorname{Odd}\left(Q_{\left(\omega_{k}\right)}\right)}{E v\left(Q_{\left(\omega_{k}\right)}\right)^{2}-\operatorname{Odd}\left(Q_{\left(\omega_{k}\right)}\right)^{2}} \\
& \tilde{F}_{1}=\operatorname{Re}\left(\frac{\tilde{Q}_{\left(\omega_{k}\right)}}{\tilde{Q}_{\left(-\omega_{k}\right)}}\right)=\frac{\operatorname{Ev}\left(\tilde{Q}_{\left(\omega_{k}\right)}\right)^{2}+\operatorname{Odd}\left(\tilde{Q}_{\left(\omega_{k}\right)}\right)^{2}}{\operatorname{Ev}\left(\tilde{Q}_{\left(\omega_{k}\right)}\right)^{2}-\operatorname{Odd}\left(\tilde{Q}_{\left(\omega_{k}\right)}\right)^{2}} \\
& \tilde{F}_{2}=\operatorname{Im}\left(\frac{\tilde{Q}_{\left(\omega_{k}\right)}}{\tilde{Q}_{\left(-\omega_{k}\right)}}\right)=\frac{2 E v\left(\tilde{Q}_{\left(\omega_{k}\right)}\right) \operatorname{Odd}\left(\tilde{Q}_{\left(\omega_{k}\right)}\right)}{E v\left(\tilde{Q}_{\left.\left(\omega_{k}\right)\right)^{2}-\operatorname{Odd}\left(\tilde{Q}_{\left(\omega_{k}\right)}\right)^{2}}\right.}
\end{aligned}
$$

$E v($ ) and $O d d($ ) correspond to the even and odd part of the polynomial, respectively. Imposing the following additional constraints ensures that the early-time ripples in transient responses are minimized 


$$
\begin{aligned}
& \boldsymbol{\kappa}_{N}-\tau_{N+1}=0 \\
& \kappa_{N-1}-\tau_{N}=0
\end{aligned}
$$

where the $\kappa$ 's and the $\tau$ 's are defined by (6.21) and (6.22) and can be expressed in terms of the $g^{\prime} s$ and $h^{\prime} s$ of (6.27) and (6.28).

The number of constraints used in (6.32) will depend on the order of the approximation. For low orders one constraint is needed. For higher order approximations additional constraints are required to control the asymptotic behavior of the high-frequency impulse response. It should be pointed out that if the of number constraints used in (6.32) are comparable to $N$ (the order of approximation) then the macromodel becomes inefficient in capturing the frequency response since it has less degrees of freedom. To obtain efficient macromodels very few constraints should be used. For the examples provided in this chapter, only one or two constraints were used, which provided significant improvement in the time-domain response.

An alternative objective function, which gives better predetermined coefficients for transmission line macromodeling than the formulation of (6.30) is to approximate

$$
e^{\left[\begin{array}{cc}
0 & j \omega \\
j \omega & 0
\end{array}\right]}=\left[\begin{array}{ll}
\cos \omega & j \sin \omega \\
j \sin \omega & \cos \omega
\end{array}\right]
$$

as

$$
\left[\begin{array}{cc}
E v(Q(\omega)) & -j \operatorname{Odd}(Q(\omega)) \\
-j \operatorname{Odd}(\tilde{Q}(\omega)) & E v(\tilde{Q}(\omega))
\end{array}\right]\left[\begin{array}{cc}
\cos \omega & j \sin \omega \\
j \sin \omega & \cos \omega
\end{array}\right] \approx\left[\begin{array}{cc}
E v(Q(\omega)) & j \operatorname{OOdd}(Q(\omega)) \\
j \operatorname{Odd}(\tilde{Q}(\omega)) & E v(\tilde{Q}(\omega))
\end{array}\right]
$$

where the coefficients of (6.27) are used to approximate the first row of (6.34) and the coefficients of (6.28) are used to approximate the second row of (6.34). Equation (6.34) is similar in form to the MRA macromodel of (6.11) and (6.12) which relies on the coefficients of (6.27) to approximate (6.11) and the coefficients of (6.28) to approximate (6.12). Inverting the matrix on the left hand side of (6.34) gives the following minimax objective 
function

$$
\begin{aligned}
& \max \left(W_{r e^{\left(\omega_{k}\right)}}\left(\cos \omega_{k}-K_{1}\right)^{2}+W_{i m^{\left(\omega_{k}\right)}}\left(\sin \omega_{k}-K_{2}\right)^{2}+\right. \\
& \left.W_{r e^{\left(\omega_{k}\right)}}\left(\cos \omega_{k}-K_{3}\right)^{2}+W_{i m^{\left(\omega_{k}\right.}}\left(\sin \omega_{k}-K_{4}\right)^{2}\right) \text { is minimum }
\end{aligned}
$$

where

$$
\begin{aligned}
& K_{1}=\frac{E v\left(Q_{\left(\omega_{k}\right)}\right) E v\left(\tilde{Q}_{\left(\omega_{k}\right)}\right)+\operatorname{Odd}\left(Q_{\left(\omega_{k}\right)}\right) \operatorname{Odd}\left(\tilde{Q}_{\left(\omega_{k}\right)}\right)}{\operatorname{Ev}\left(Q_{\left(\omega_{k}\right)}\right) \operatorname{Ev}\left(\tilde{Q}_{\left(\omega_{k}\right)}\right)-\operatorname{Odd}\left(Q_{\left(\omega_{k}\right)}\right) \operatorname{Odd}\left(\tilde{Q}_{\left(\omega_{k}\right)}\right)} \\
& K_{2}= \frac{2 E v\left(\tilde{Q}_{\left(\omega_{k}\right)}\right) \operatorname{Odd}\left(Q_{\left(\omega_{k}\right)}\right)}{\operatorname{Ev}\left(Q_{\left(\omega_{k}\right)}\right) \operatorname{Ev}\left(\tilde{Q}_{\left(\omega_{k}\right)}\right)-\operatorname{Odd}\left(Q_{\left(\omega_{k}\right)}\right) \operatorname{Odd}\left(\tilde{Q}_{\left(\omega_{k}\right)}\right)} \\
& K_{3}=\frac{\operatorname{Ev}\left(Q_{\left(\omega_{k}\right)}\right) \operatorname{Ev}\left(\tilde{Q}_{\left(\omega_{k}\right)}\right)+\operatorname{Odd}\left(Q_{\left(\omega_{k}\right)}\right) \operatorname{Odd}\left(\tilde{Q}_{\left(\omega_{k}\right)}\right)}{\operatorname{Ev}\left(Q_{\left(\omega_{k}\right)}\right) E v\left(\tilde{Q}_{\left(\omega_{k}\right)}\right)-\operatorname{Odd}\left(Q_{\left(\omega_{k}\right)}\right) \operatorname{Odd}\left(\tilde{Q}_{\left(\omega_{k}\right)}\right)} \\
& K_{4}=\frac{2 E v\left(Q_{\left(\omega_{k}\right)}\right) \operatorname{Odd}\left(\tilde{Q}_{\left(\omega_{k}\right)}\right)}{\operatorname{Ev}\left(Q_{\left(\omega_{k}\right)}\right) E v\left(\tilde{Q}_{\left(\omega_{k}\right)}\right)-\operatorname{Odd}\left(Q_{\left(\omega_{k}\right)}\right) \operatorname{Odd}\left(\tilde{Q}_{\left(\omega_{k}\right)}\right)}
\end{aligned}
$$

Imposing the constraints of (6.32) and ensuring that $g_{0, i}, g_{1, i}, h_{0, i}, h_{1, i}>0$ for all values of $i$ will minimize early-time ripples in transient responses and guarantee the passivity of the macromodel.

The predetermined coefficients given by (6.35) produce better time-domain macromodels than (6.30), since the formulation couples the two scalar approximations in (6.36) to minimize the overall error in the MRA macromodel. For two-conductor transmission line and without loss of generality, the relationship of the terminal voltages and currents of the MRA macromodel given by (6.11) and (6.12) can be expressed as

$$
\begin{gathered}
{\left[\begin{array}{ll}
Q_{11} & Q_{12} \\
\tilde{Q}_{21} & \tilde{Q}_{22}
\end{array}\right]\left[\begin{array}{c}
V(d, s) \\
-I(d, s)
\end{array}\right] \approx\left[\begin{array}{cc}
Q_{11} & -Q_{12} \\
\tilde{Q}_{21} & \tilde{Q}_{22}
\end{array}\right]\left[\begin{array}{l}
V(0, s) \\
I(0, s)
\end{array}\right]} \\
{\left[\begin{array}{c}
V(d, s) \\
-I(d, s)
\end{array}\right] \approx\left[\begin{array}{cc}
\frac{Q_{11} \tilde{Q}_{22}+Q_{12} \tilde{Q}_{21}}{Q_{11} \tilde{Q}_{22}-Q_{12} \tilde{Q}_{21}} & \frac{-2 \tilde{Q}_{22} Q_{12}}{Q_{11} \tilde{Q}_{22}-Q_{12} \tilde{Q}_{21}} \\
\frac{-2 Q_{11} \tilde{Q}_{21}}{Q_{11} \tilde{Q}_{22}-Q_{12} \tilde{Q}_{21}} & \frac{Q_{11} \tilde{Q}_{22}+Q_{12} \tilde{Q}_{21}}{Q_{11} \tilde{Q}_{22}-Q_{12} \tilde{Q}_{21}}
\end{array}\right]\left[\begin{array}{l}
V(0, s) \\
I(0, s)
\end{array}\right]}
\end{gathered}
$$


The following relationship exists between (6.36) and (6.37),

$$
\begin{gathered}
Q_{11} \tilde{Q}_{22}+Q_{12} \tilde{Q}_{21}=\sum_{i=0} \mu_{i}(a b)^{i} \\
E v\left(Q_{(s)}\right) E v\left(\tilde{Q}_{(s)}\right)+\operatorname{Odd}\left(Q_{(s)}\right) \operatorname{Odd}\left(\tilde{Q}_{(s)}\right)=\sum_{i=0} \mu_{i}(s)^{2 i} \\
Q_{11} \tilde{Q}_{22}-Q_{12} \tilde{Q}_{21}=\sum_{i=0} \gamma_{i}(a b)^{i} \\
E v\left(Q_{(s)}\right) E v\left(\tilde{Q}_{(s)}\right)-\operatorname{Odd}\left(Q_{(s)}\right) \operatorname{Odd}\left(\tilde{Q}_{(s)}\right)=\sum_{i=0} \gamma_{i}(s)^{2 i} \\
\tilde{Q}_{22} Q_{12}=\sum_{i=0} \chi_{i}(a b)^{i} a \\
E v\left(\tilde{Q}_{(s)}\right) \operatorname{Odd}\left(Q_{(s)}\right)=\sum_{i=0} \chi_{i}(s)^{2 i} s \\
Q_{11} \tilde{Q}_{21}=\sum_{i=0} \varphi_{i}(a b)^{i} b \\
E v\left(Q_{(s)} \operatorname{Odd}\left(\tilde{Q}_{(s)}\right)=\sum_{i=0} \varphi_{i}(s)^{2 i} s\right.
\end{gathered}
$$

where $a=(R+s L) d, b=(G+s C) d$ and $\mu_{i}, \gamma_{i}, \chi_{i}$ and $\varphi_{i}$ are the predetermined coefficients given by the scalar approximations of (6.27) and (6.28). The error functions minimized by (6.35) are with respect to $\mu_{i}, \gamma_{i}, \chi_{i}$ and $\varphi_{i}$, which are the predetermined coefficients used by (6.37)-(6.41). This produces more accurate macromodels for (6.11)-(6.12) when compared to the formulation of $(6.30)$.

It should be emphasized that the minimax objective functions provided in this section are independent of the number of coupled lines and the p.u.l. parameters. The results obtained are then stored and the macromodel can be formulated analytically in terms of the predetermined coefficients and p.u.l. parameters.

\subsubsection{Preserving Passivity}

In chapter 3, it was shown that if the conditions of Theorem 3.1 are satisfied, then the matrices $\Psi_{1}$ and $\Psi_{2}$ of (3.13) are both positive real, which is necessary to prove passivity. 
The rational function $\boldsymbol{H}_{w}$ is formulated using the predetermined coefficients of (6.13). Since it is assumed that (6.8) satisfies the Hurwitz conditions of Theorem 3.1, it can be shown that $\Psi_{1}$ is positive real as described in Chapter 3. Similarly, the rational function $\tilde{\boldsymbol{H}}_{x}$ is formulated by the predetermined coefficients of (6.9). Since it is assumed that (6.9) also satisfies the Hurwitz conditions of Theorem 3.1, it can be shown that $\Psi_{2}$ is also positive real. The summation of two positive real matrices results in a positive real matrix, which proves that the new macromodel is passive.

\subsection{Development of Time-Domain Transmission Line Macromodel}

To control the asymptotic behavior of the high-frequency impulse response, the MRA macromodel of (6.11) and (6.12) relies on constraint optimization to obtain the predetermined scalar coefficients of (6.8) and (6.9). The Padé coefficients of (3.24) cannot be used since they are fixed values and do not satisfy the conditions of (6.32).

In chapter 4, the MRA macromodel is expressed as a product of low order rational functions. This formulation cannot be used for the new MRA macromodel since the method relies on two scalar approximations (i.e. (6.8) and (6.9)). This section describes an alternative time-domain formulation for the MRA macromodel. The proposed approach yields diagonally dominant, band limited and highly sparse matrices. In addition, the macromodel is formulated analytically in terms of predetermined coefficients and the p.u.l. parameters.

\subsubsection{Formulation of Time-Domain Macromodel}

The time-domain macromodel is realized from the Y-parameters equations of (6.14). The matrices $\Psi_{1}$ and $\Psi_{2}$ of (6.14) are realized separately, since they are derived from two different scalar approximations. To realize $\Psi_{1}$ in terms of subsections that are in series, $\boldsymbol{H}_{\boldsymbol{w}}$ term is expressed as 


$$
\Psi_{1}=\left[\begin{array}{cc}
\boldsymbol{H}_{w} & -\boldsymbol{H}_{w} \\
-\boldsymbol{H}_{w} & \boldsymbol{H}_{w}
\end{array}\right] \quad \boldsymbol{H}_{w}=\frac{1}{2}\left[\boldsymbol{Q}_{12}\right]^{-1} \boldsymbol{Q}_{11}=\left(\sum_{i=i}^{N / 2}\left(\eta_{1, i} \boldsymbol{a}_{+}\left(\phi_{1, i} \boldsymbol{b}\right)^{-1}\right)^{-1}\right)^{-1}
$$

where $\boldsymbol{Q}_{12}$ and $\boldsymbol{Q}_{11}$ are defined by (6.13); $\eta_{1, i}$ and $\phi_{1, i}$ are predetermined coefficients and are obtained from the scalar approximation of (6.8) as

$$
\frac{\operatorname{Odd}(Q(x))}{E v(Q(x))}=\sum_{i=1}^{N / 2}\left(\frac{\phi_{1, i} x}{\phi_{1, i} \eta_{1, i} x^{2}+1}\right)
$$

$\operatorname{Odd}(\mathrm{)})$ and $E v($ ) correspond to the even and odd part of the polynomial, respectively; $\boldsymbol{a}=(\boldsymbol{R}(s)+s \boldsymbol{L}(s)) d$ and $\boldsymbol{b}=(\boldsymbol{G}(s)+s \boldsymbol{L}(s)) d$. Next, to realize $\Psi_{2}$ in terms of subsections that are in series, $\tilde{\boldsymbol{H}}_{\boldsymbol{x}}$ term is expressed as

$$
\Psi_{2}=\left[\begin{array}{cc}
\tilde{\boldsymbol{H}}_{x} & \tilde{\boldsymbol{H}}_{x} \\
\tilde{\boldsymbol{H}}_{x} & \tilde{\boldsymbol{H}}_{x}
\end{array}\right] \quad \tilde{\boldsymbol{H}}_{x}=\frac{1}{2}\left[\tilde{\boldsymbol{Q}}_{22}\right]^{-1} \tilde{\boldsymbol{Q}}_{21}=\left(\left(\phi_{2,0} \boldsymbol{b}\right)^{-1}+\sum_{i=1}^{N / 2}\left(\eta_{2, i} \boldsymbol{a}+\left(\phi_{2, i} \boldsymbol{b}\right)^{-1}\right)^{-1}\right)^{-1}
$$

where $\tilde{\boldsymbol{Q}}_{21}$ and $\tilde{\boldsymbol{Q}}_{22}$ are defined by (6.13); $\eta_{2, i}$ and $\phi_{2, i}$ are predetermined coefficients and are obtained from the scalar approximation of (6.9) as

$$
\frac{E v(\tilde{Q}(x))}{\operatorname{Odd}(\tilde{Q}(x))}=\frac{1}{\phi_{2,0} x}+\sum_{i=1}^{N / 2}\left(\frac{\phi_{2, i} x}{\phi_{2, i} \eta_{2, i} x^{2}+1}\right)
$$

Equations (6.42) and (6.44) enables the realization of $\Psi_{1}$ and $\Psi_{2}$ in terms of cascaded subsections. The circuit description of $\Psi_{1}$ and $\Psi_{2}$ is provided in the next section.

\subsubsection{Circuit Realization of MRA Macromodel}

In this section, the time-domain macromodel is obtained for transmission lines with frequency-dependent parameters. The circuit realization of the macromodel is derived from (6.42) and (6.44). The basic circuit topology of (6.42) and (6.44) is shown in Figure 6.1. The circuit description of each subsection for a two-conductor transmission line is shown in Figure 6.2 and Figure 6.3. Figure 6.2 represents the circuit realization of $\Psi_{1}$ as described by (6.42) and Figure 6.3 represents the circuit realization of $\Psi_{2}$ as described by 
(6.44). The variable $\lambda$ and $\mu$ in Figure 6.2 and Figure 6.3 correspond to the number of sections required to model the frequency-dependent parameters. If $\lambda=\mu=1$, then the circuit realization of Figure 6.2 and Figure 6.3 is equivalent to the frequency-independent parameter case. Circuit representations for the $\mathrm{RC}$ and lossless transmission lines can also be derived from (6.42) and (6.44). The procedure is similar to the RC and lossless circuit realization described in Chapter 4.

The circuit realization of transmission lines with frequency-dependent parameters can also be extended to MTLs. An example circuit description of a three-conductor transmission line is shown in Figure 6.4 and Figure 6.5. Figure 6.4 represents the circuit realization of $\Psi_{1}$ as described by (6.42) and Figure 6.5 represents the circuit realization of $\Psi_{2}$ as described by (6.44).

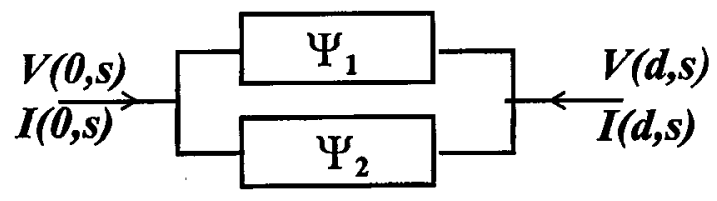

(a) MRA macromodel.

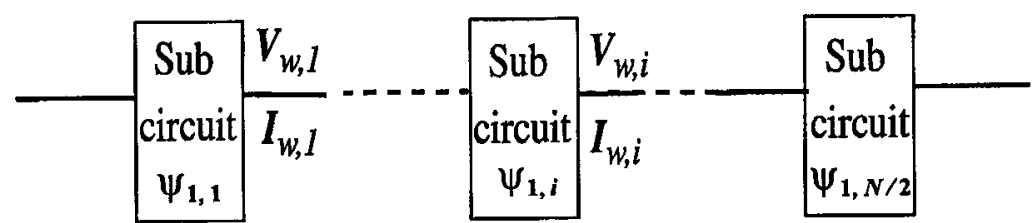

b) $\Psi_{1}$ represented in terms of the subsections of (6.42)

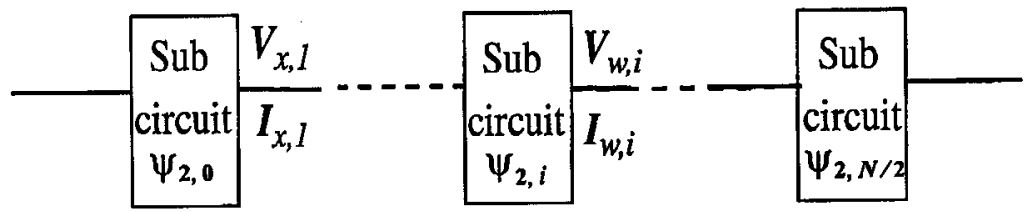

c) $\Psi_{2}$ represented in terms of the subsections of (6.44)

Figure 6.1 MRA macromodel representation 
It should be noted that the circuit realization presented in this section is similar to the method given in Chapter 4 and 5. For the case of frequency-independent parameters, both macromodels are obtained analytically in term of predetermined coefficients (i.e. $\eta_{1, i}$, $\eta_{2, i}, \phi_{1, i}$ and $\left.\phi_{2, i}\right)$ and the p.u.l. parameters. For the case frequency-dependent parameters, with the knowledge of the circuit configurations of the p.u.l. parameters (i.e. Figure 5.4 to Figure 5.7) the formulation is also analytic in terms of predetermined coefficients and the circuit values of p.u.l. parameters. In addition, the circuit realization provided in this section can also be used for the MRA macromodel described in Chapter 3.

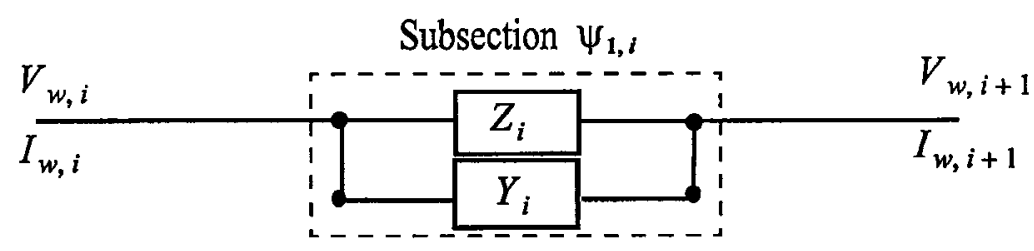

a) Subsection $\psi_{1, i}$ of Figure $6.1 \mathrm{~b}(i=[1,2, \ldots, N / 2])$

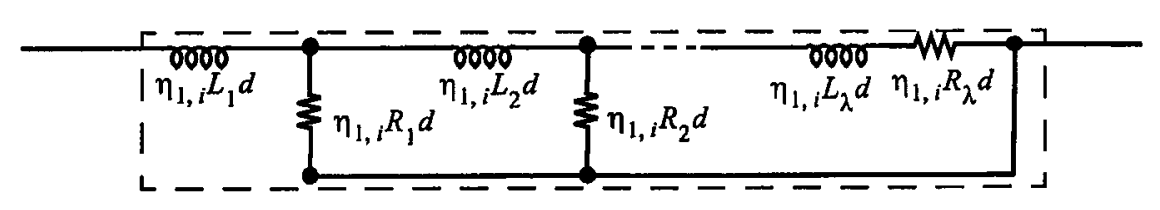

b) $Z_{i}$ circuit (RL canonical configuration)

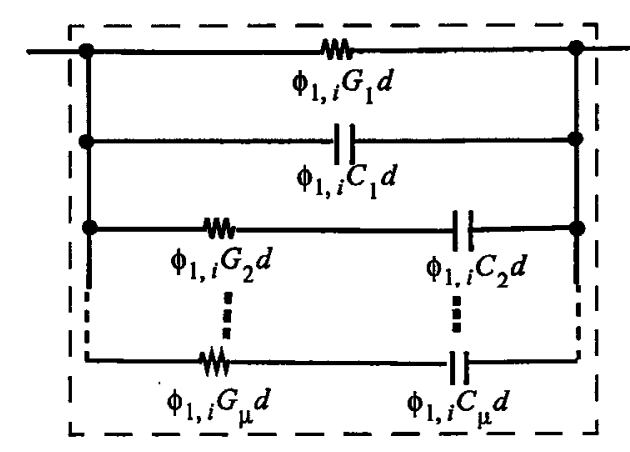

c) $Y_{i}$ circuit (RC Foster configuration)

Figure 6.2 Realization of two-conductor transmission-line for (6.42) 


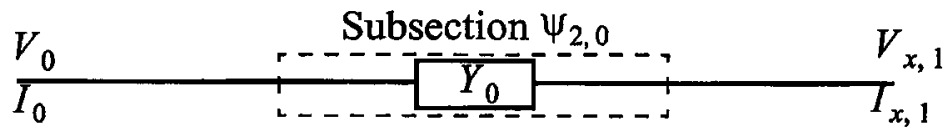

a) Subsection $\psi_{2,0}$ of Figure 6.1c
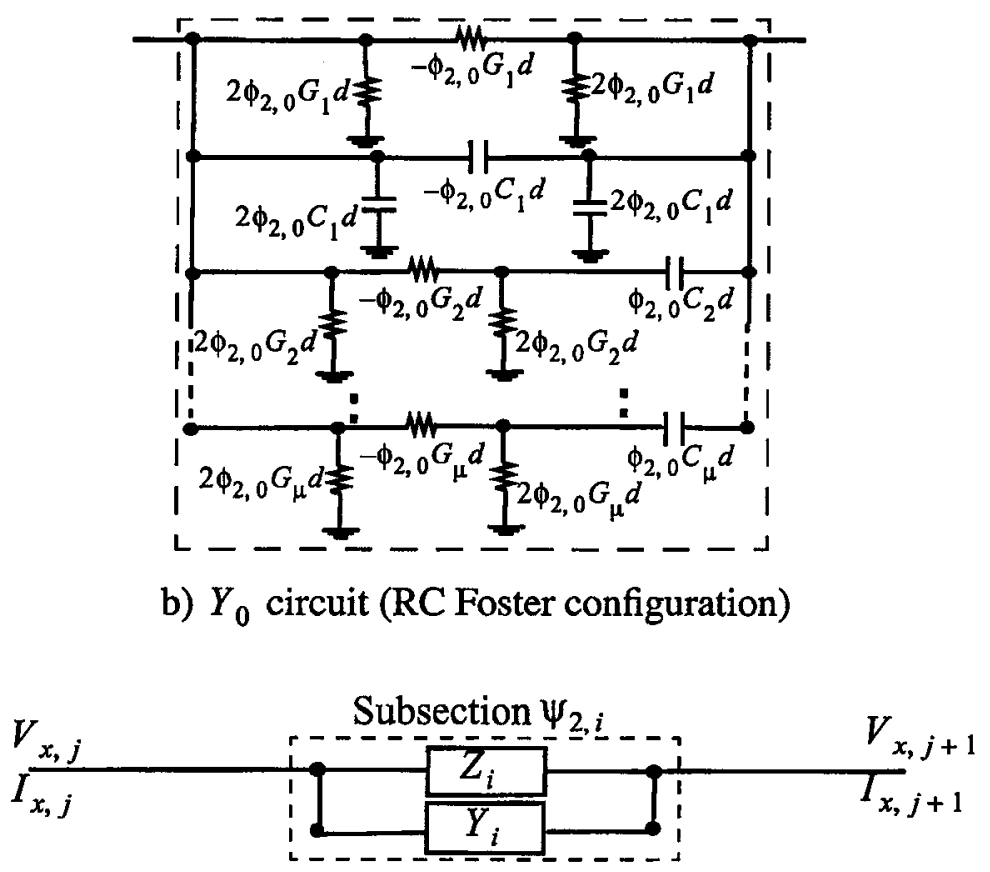

c) Subsection $\psi_{2, i}$ of Figure $6.1 \mathrm{c}(i=[1,2, \ldots, N / 2])$

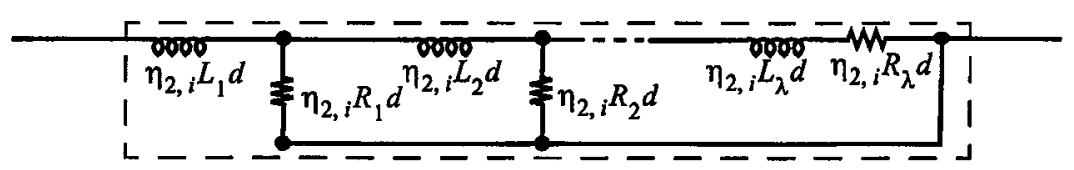

d) $Z_{i}$ circuit (RL canonical configuration)

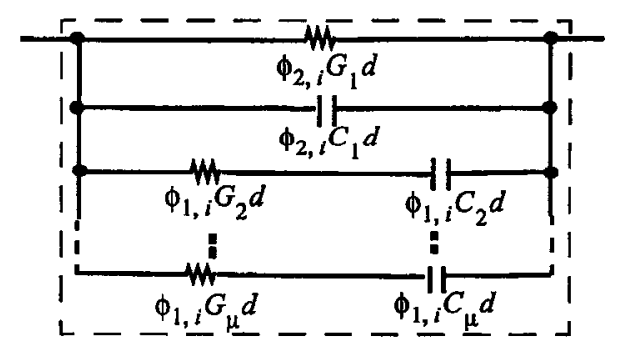

e) $Y_{i}$ circuit (RC Foster configuration)

Figure 6.3 Realization of two-conductor transmission-line for (6.44) 
Subsection $\psi_{1, i}$

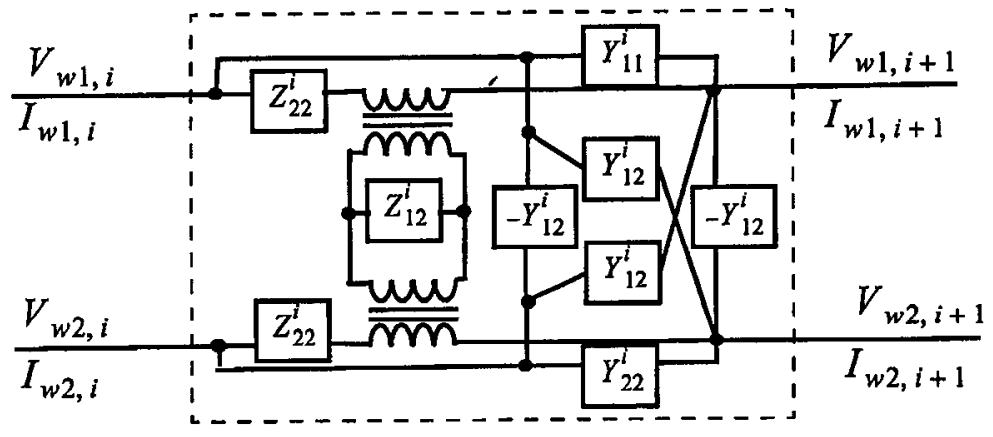

a) Subsection $\psi_{1, i}$ of Figure $6.1 \mathrm{~b}(i=[1,2, \ldots, N / 2])$

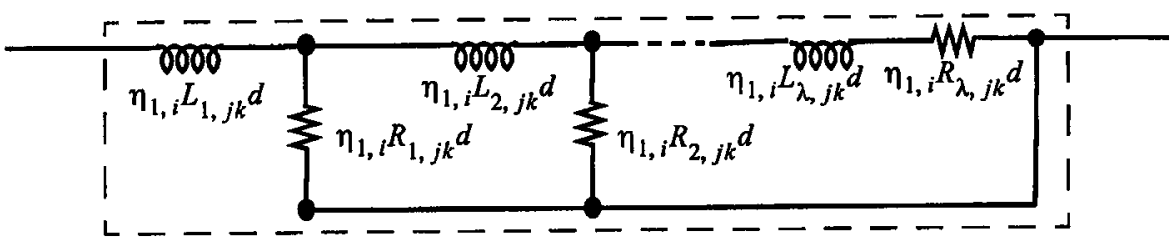

b) $Z_{j k}^{i}$ circuit (RL canonical configuration)

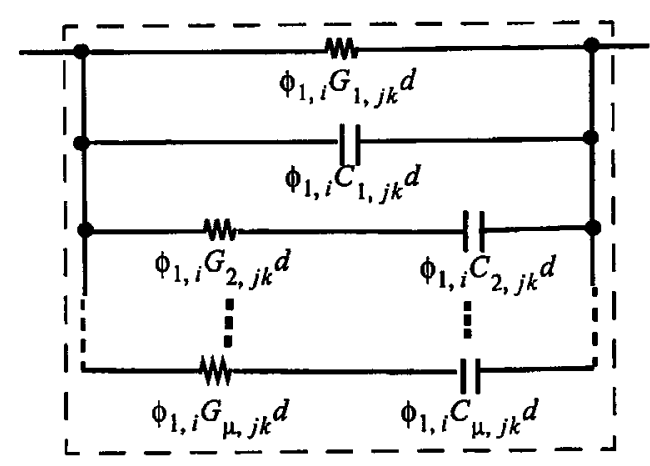

c) $Y_{j k}^{i}$ circuit (RC Foster configuration)

Figure 6.4 Realization of MTL for (6.42) 


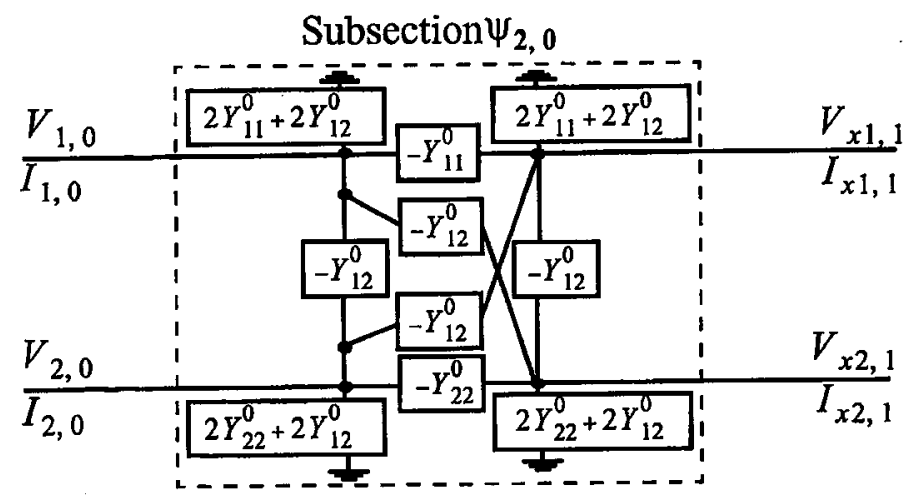

a) Subsection $\psi_{2,0}$ of Figure 6.1c

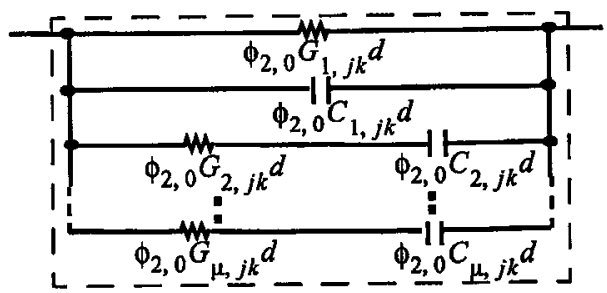

b) $Y_{i, k}^{0}$ circuit (RC Foster configuration)

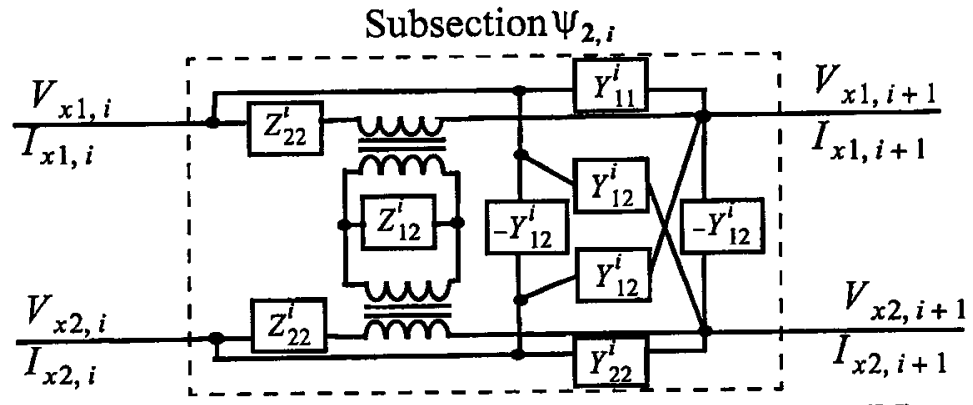

c) Subsection $\psi_{2, i}$ for Figure $6.1 \mathrm{c}(i=[1,2, \ldots, N / 2])$
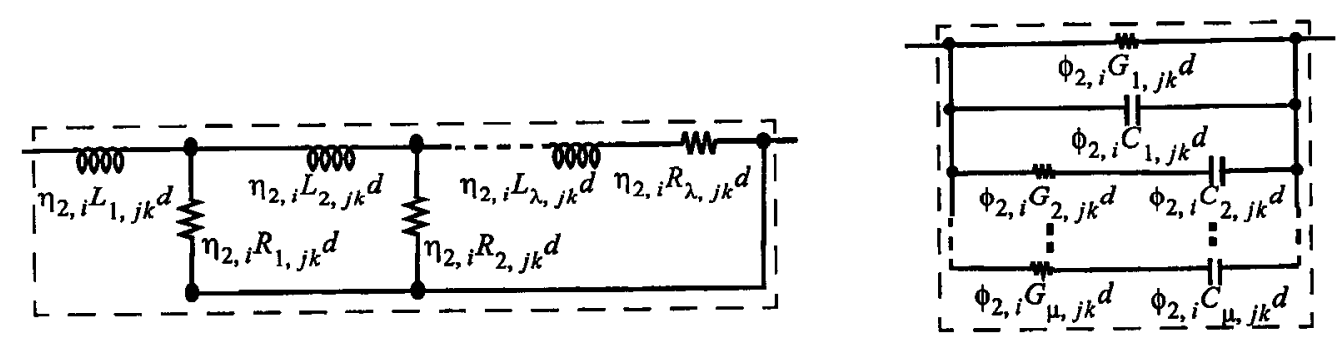

d) $Z_{j k}^{i}$ circuit (RL canonical configuration)

e) $Y_{j k}^{i}$ circuit (RC Foster configuration)

Figure 6.5 Realization of MTL for (6.44) 


\subsection{Examples}

In this section three examples are presented to demonstrate the validity of the proposed macromodel.

\subsubsection{Example 6.1}

In this example, a long lossy distributed transmission line network is analyzed with PRIMA [13] and the proposed algorithm. The near end is connected to a voltage source through a $5 \Omega$ resistor and the far end is terminated with a $500 \Omega$ resistor. The line is $40 \mathrm{~cm}$ long and the p.u.1. parameters are $R=1.93 \Omega / \mathrm{cm}, L=2.97 \mathrm{nH} / \mathrm{cm}$, $G=79 \mathrm{nS} / \mathrm{cm}, C=1.61 \mathrm{pF} / \mathrm{cm}$. The frequency response at the far end of the transmission line (Vout) is given in Figure 6.6. Both approaches match the "Exact" response up to $2.5 \mathrm{GHz}$, accurately, however, the high-frequency behavior beyond the matched frequency of $2.5 \mathrm{GHz}$ is significantly different. Within the context of this section "Exact" response refers to solving Telegrapher's equations analytically through eigenvalue/ eigenvector analysis [2]. Note that, the proposed macromodel contains only 31 poles, where as the PRIMA macromodel contains 50 poles (an equivalent 31 pole PRIMA macromodel matches the "Exact" response up to $1.6 \mathrm{GHz}$ only). The transient response corresponding to the PRIMA macromodel is given in Figure 6.7 (the input is a pulse with $0.35 \mathrm{~ns}$ rise/fall time and $1 \mathrm{~ns}$ pulse width; the label "IFFT" refers to inverse fast Fourier transform of "Exact" response multiplied by the input frequency spectrum). It can be noticed that the flat delay portion of the response from PRIMA suffers from spurious ripples. Figure 6.8 gives the transient response of the proposed macromodel and it can be noticed that the spurious ripples in the flat-delay portion are significantly minimized. The simulations were obtained using HSPICE [93] and circuit equivalent realization of macromodels, on a SUN Blade-1000 workstation with $900 \mathrm{MHz}$ UltraSPARC-III CPU. For this example, the predetermined coefficients of the proposed algorithm were obtained using two constraints (i.e. $\kappa_{N}=\tau_{N+1}, \kappa_{N-1}=\tau_{N}$ in equation (6.32)). 


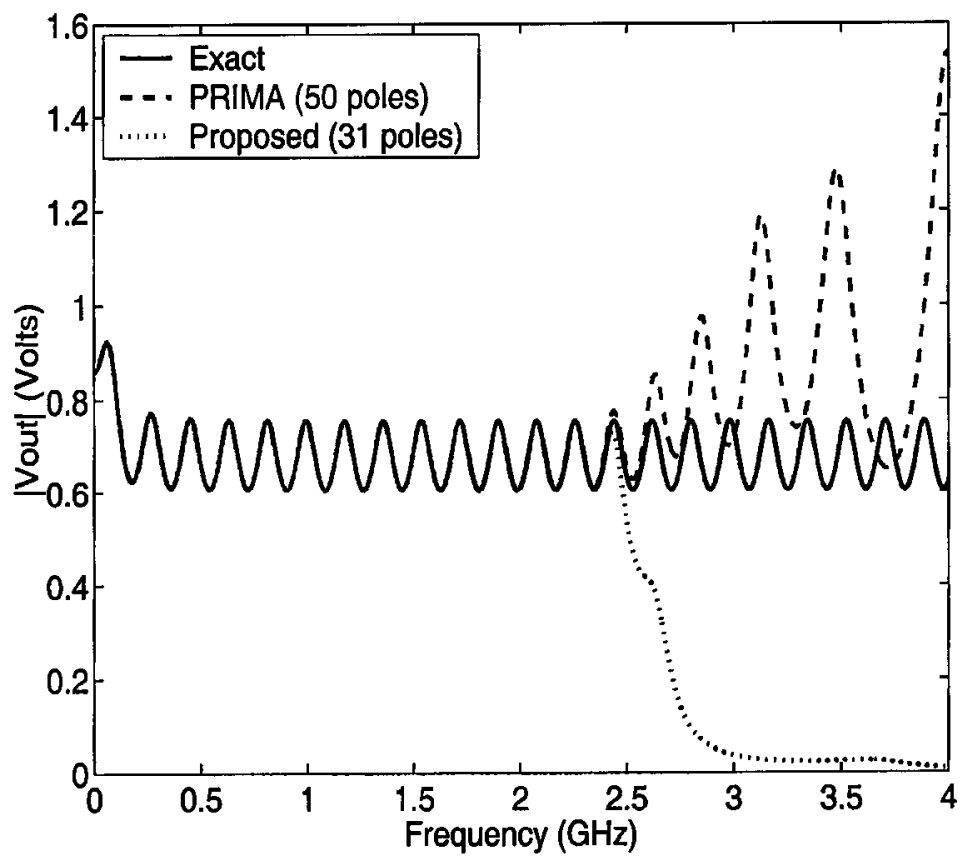

Figure 6.6 Frequency response (Example 6.1)

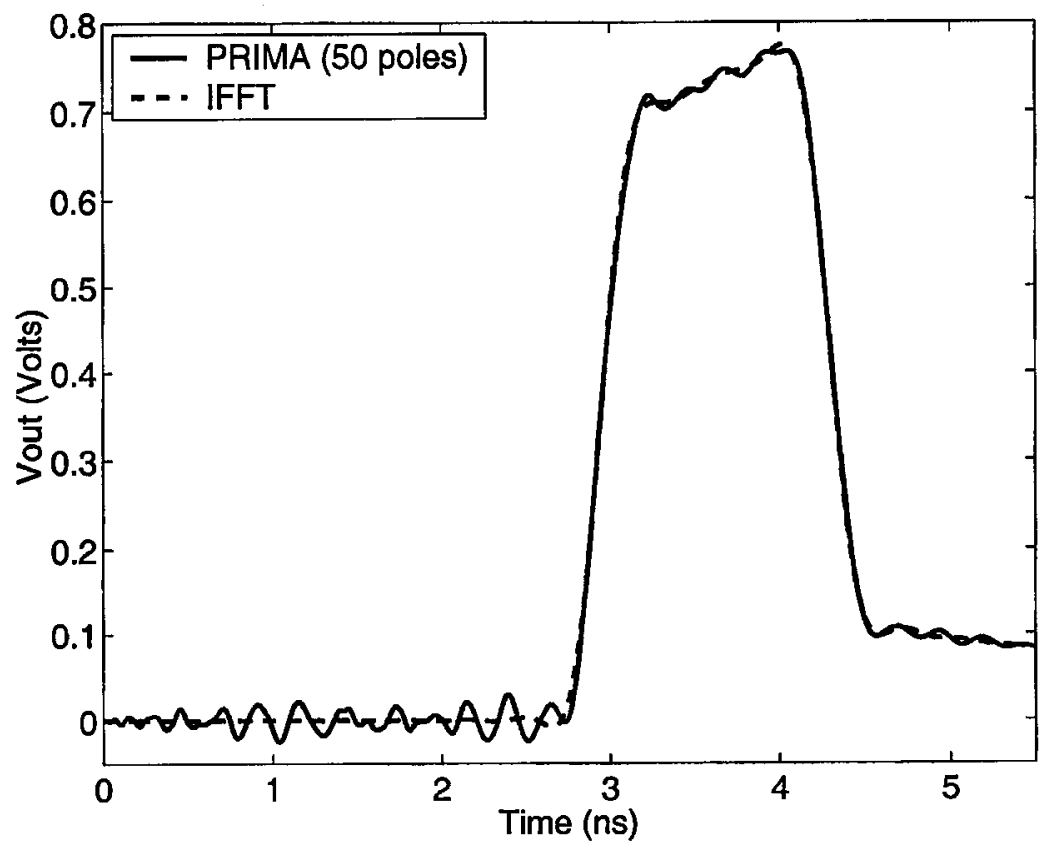

Figure 6.7 Transient response IFFT versus PRIMA (Example 6.1) 


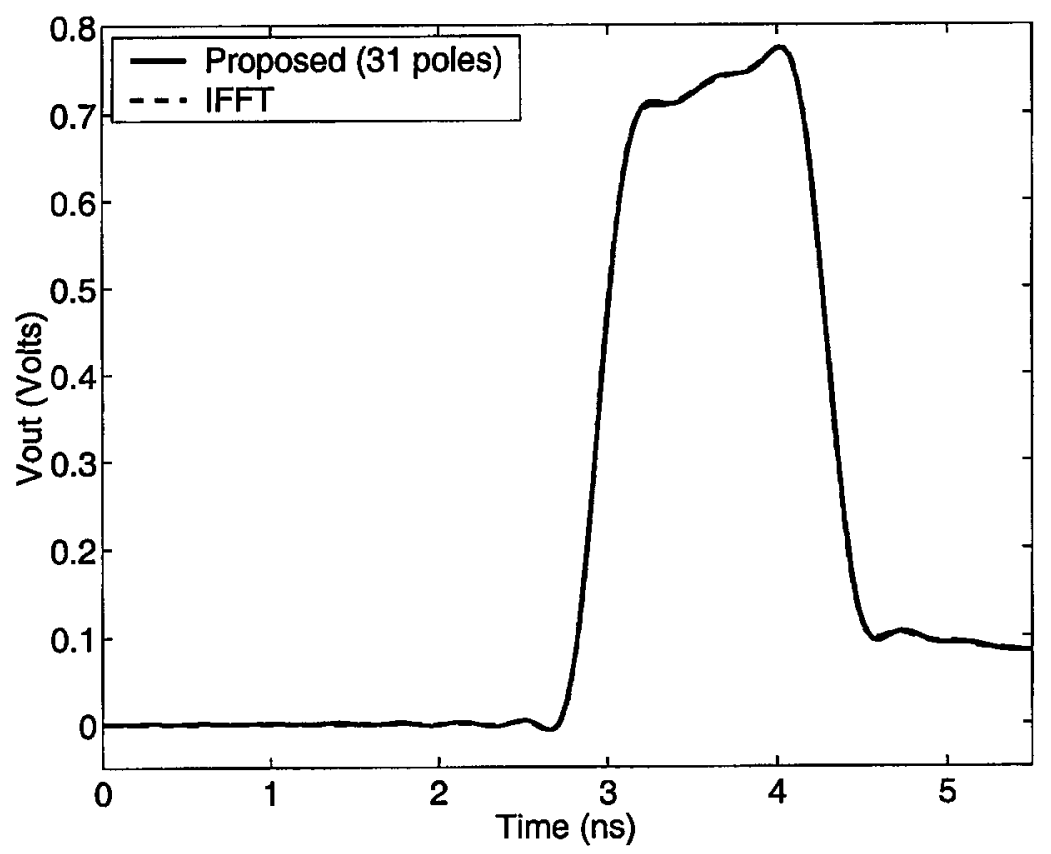

Figure 6.8 Transient response IFFT versus proposed macromodel (Example 6.1)

\subsubsection{Example 6.2}

A network with lossy-coupled transmission lines with frequency-dependent parameters is considered in Figure 6.9. The length of the transmission line network is $0.5 \mathrm{~cm}$ and the p.u.1. parameters are described in Table 6.1. The frequency dependent data are modeled using $2 \mathrm{RL}$ sections for $R_{I I}(s)+s L_{11}(s), 2 \mathrm{RL}$ sections for $R_{22}(s)+s L_{22}(s)$ and $3 \mathrm{RL}$ sections for $R_{12}(s)+\mathrm{s} L_{12}(s)$. The time-domain responses corresponding to an input step of $1 \mathrm{~V}$ and rise time of $0.07 \mathrm{~ns}$ at the victim node $\mathrm{V}_{4}$, using the MRA macromodel of Chapter 5 and the proposed algorithm are given in Figure 6.10 and Figure 6.11, respectively. The label "MoC" refers to the method of characteristics macromodel as implemented in [66]. As seen, while matching the response accurately, the proposed algorithm minimized the early-time spurious ripples, considerably. For this example, the CPU time of the proposed method was 0.13 seconds, while the method of characteristics required 1.36 seconds. The simulations were obtained using PowerSpice on a RS6000 44P model 170 64-bit machine 
with Power 3-II microprocessor at $400 \mathrm{MHz}$. The predetermined coefficients of the proposed algorithm were obtained using two constraints (i.e. $\kappa_{N}=\tau_{N+1}, \kappa_{N-1}=\tau_{N}$ in equation (6.32)).

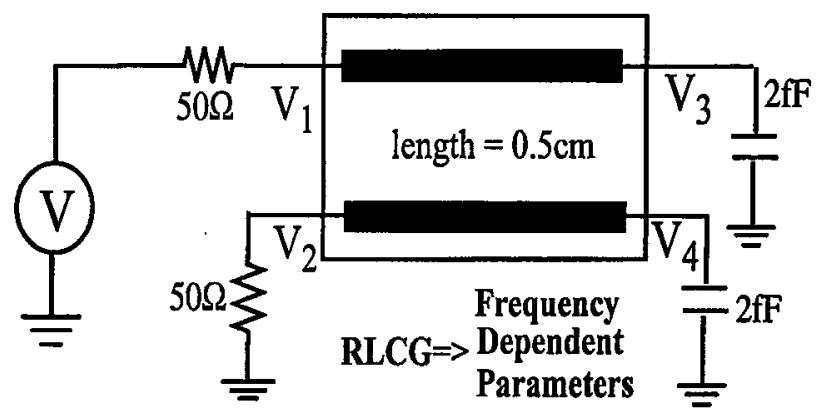

Figure 6.9 Lossy coupled transmission-line network (Example 6.2)

\begin{tabular}{c|c|c|c|c|c|c}
\hline $\begin{array}{c}\text { Freq } \\
(\mathrm{GHz})\end{array}$ & $\begin{array}{c}\mathrm{R}_{11} \\
(\Omega / \mathrm{cm})\end{array}$ & $\begin{array}{c}\mathrm{R}_{22} \\
(\Omega / \mathrm{cm})\end{array}$ & $\begin{array}{c}\mathrm{R}_{12} \\
(\Omega / \mathrm{cm})\end{array}$ & $\begin{array}{c}\mathrm{L}_{11} \\
(\mathrm{nH} / \mathrm{cm})\end{array}$ & $\begin{array}{c}\mathrm{L}_{22} \\
(\mathrm{nH} / \mathrm{cm})\end{array}$ & $\begin{array}{c}\mathrm{L}_{12} \\
(\mathrm{nH} / \mathrm{cm})\end{array}$ \\
\hline 0 & 54.980 & 333.700 & 0 & 14.024 & 14.1697 & 11.186 \\
\hline $1.0 \mathrm{e}-3$ & 56.440 & 335.550 & 1.4094 & 14.024 & 14.1697 & 11.186 \\
\hline $3.3 \mathrm{e}-3$ & 56.442 & 335.550 & 1.4130 & 14.023 & 14.1697 & 11.186 \\
\hline $6.6 \mathrm{e}-3$ & 56.447 & 335.556 & 1.4220 & 14.020 & 14.1607 & 11.178 \\
\hline $1.0 \mathrm{e}-2$ & 56.460 & 335.570 & 1.4320 & 14.000 & 14.1530 & 11.167 \\
\hline $3.3 \mathrm{e}-2$ & 56.677 & 335.785 & 1.4408 & 13.840 & 14.0030 & 11.012 \\
\hline $6.6 \mathrm{e}-2$ & 57.326 & 336.430 & 2.0620 & 13.365 & 13.5740 & 10.557 \\
\hline 0.1 & 58.260 & 337.360 & 2.9540 & 12.710 & 12.9820 & 9.930 \\
\hline 0.3 & 63.800 & 342.875 & 8.2570 & 9.3000 & 9.9040 & 6.676 \\
\hline 0.66 & 68.337 & 347.375 & 12.560 & 7.1279 & 7.9456 & 4.603 \\
\hline 1.0 & 70.375 & 349.380 & 14.454 & 6.5050 & 7.3840 & 4.009 \\
\hline 3.5 & 77.443 & 356.416 & 20.320 & 5.6750 & 6.6450 & 3.226 \\
\hline 6.6 & 84.188 & 363.250 & 24.549 & 5.5130 & 6.4960 & 3.087 \\
\hline 10.0 & 92.682 & 371.725 & 29.190 & 5.4160 & 6.4090 & 3.018 \\
\hline \multicolumn{6}{c}{$R_{12}=R_{21}$} \\
\multicolumn{5}{c}{$L_{12}=L_{21}$} \\
\end{tabular}

Table 6.1 P.u.l. parameters of Example 6.2 


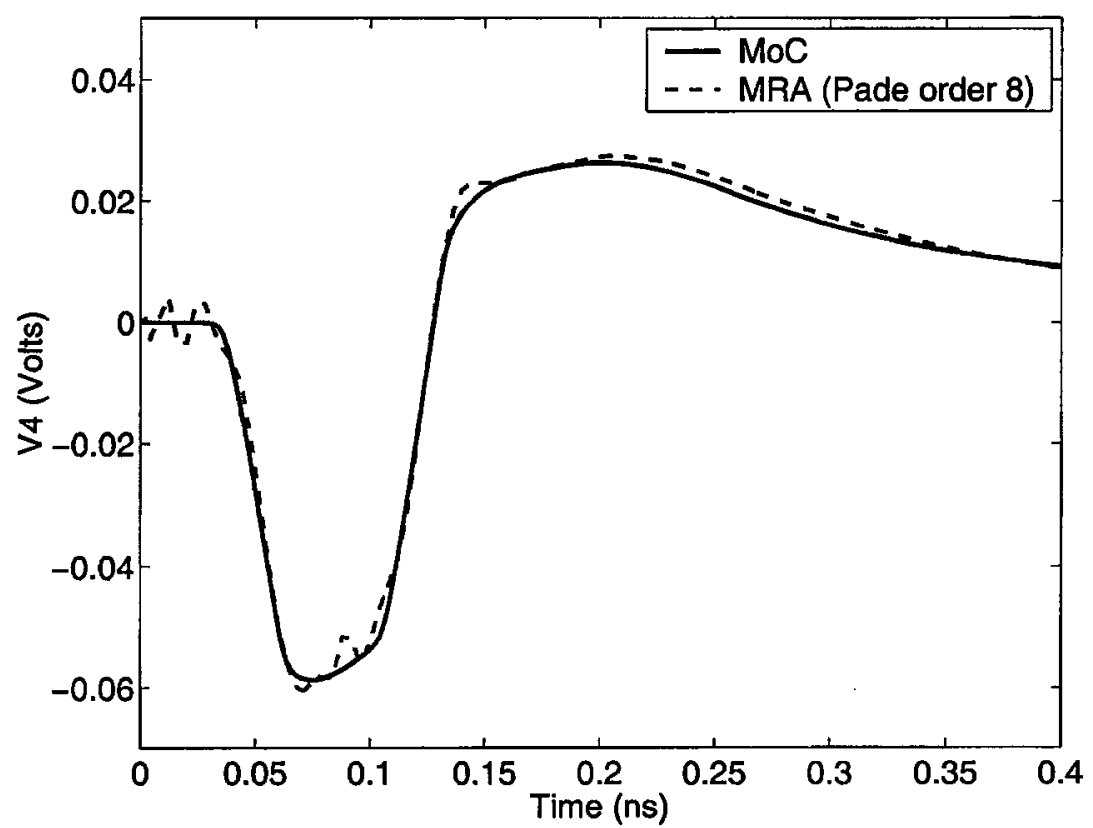

Figure 6.10 Transient response, method of characteristics vs MRA (Pade coefficients) (Example 6.2)

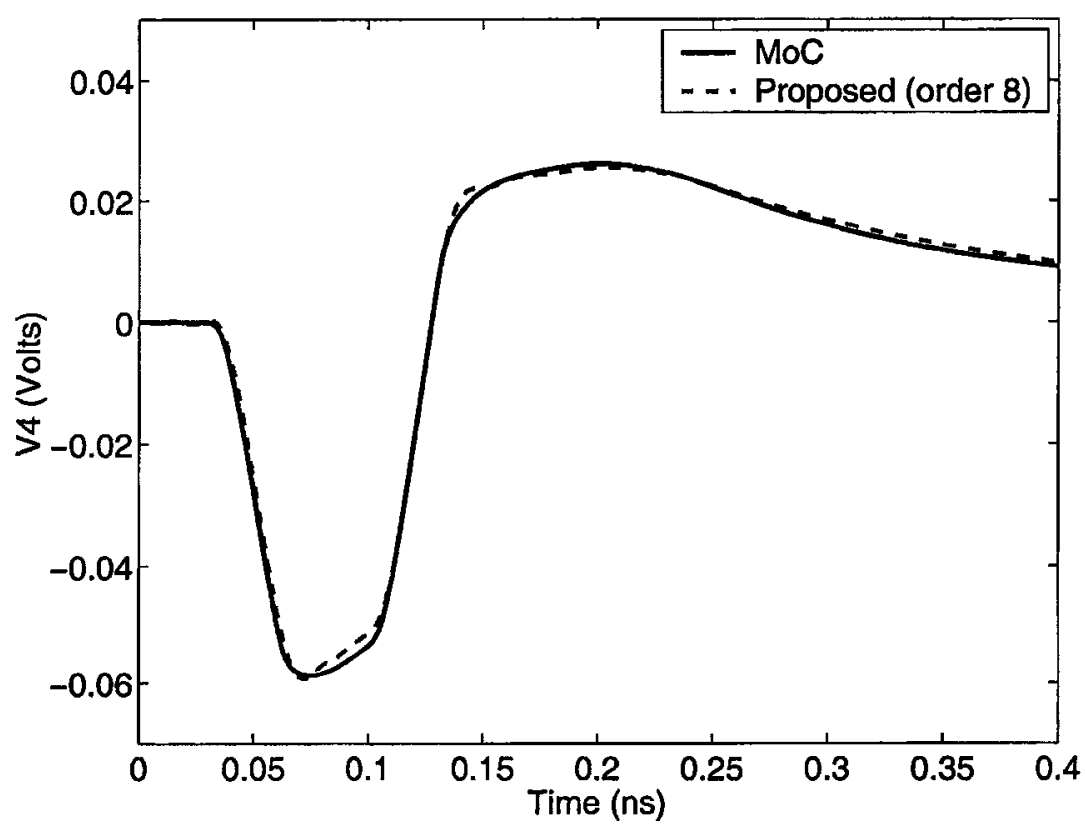

Figure 6.11 Transient response, method of characteristics vs proposed (Example 6.2) 


\subsubsection{Example 6.3}

A network with a long transmission line with frequency dependent parameters is considered in Figure 6.12 [95]. The length of the transmission line network is $10 \mathrm{~m}$ long and the per-unit-length parameters are described in Table 6.2. The frequency dependent data are modeled using $4 \mathrm{RL}$ sections for $R(s)+s L(s)$ and $4 \mathrm{RC}$ sections for $G(s)+\mathrm{s} C(s)$. The time domain responses at node V1 and V2 corresponding to a unit step input with 0.1 ns time rise are shown in Figure 6.13. For their respective orders, both the MRA macromodel (680 sections $=40$ segments of order $\mathrm{N}=17$ ) and the conventional lumped model (4000 sections) are in agreement. However, the MRA algorithm introduces $83 \%$ fewer variables to the MNA equations compared to the lumped segmentation model.

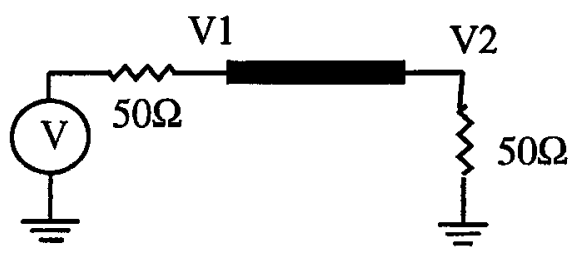

Figure 6.12 Long low loss line with frequency dependent parameters (Example 6.3)

\begin{tabular}{c|c|c|c|c}
\hline $\begin{array}{c}\text { Freq } \\
(\mathrm{GHz})\end{array}$ & $\begin{array}{c}\mathrm{R} \\
(\Omega / \mathrm{cm})\end{array}$ & $\begin{array}{c}\mathrm{L} \\
(\mathrm{nH} / \mathrm{cm})\end{array}$ & $\begin{array}{c}\mathrm{G} \\
(\mathrm{mS} / \mathrm{cm})\end{array}$ & $\begin{array}{c}\mathrm{C} \\
(\mathrm{pF} / \mathrm{cm})\end{array}$ \\
\hline $1 \mathrm{e}-4$ & $1.86 \mathrm{e}-3$ & 2.778 & $4.047 \mathrm{e}-7$ & 0.8645 \\
\hline $1.0 \mathrm{e}-3$ & $3.29 \mathrm{e}-3$ & 2.539 & $4.047 \mathrm{e}-6$ & 0.8636 \\
\hline $4.64 \mathrm{e}-3$ & $6.51 \mathrm{e}-3$ & 2.310 & $1.879 \mathrm{e}-5$ & 0.8629 \\
\hline $1.0 \mathrm{e}-2$ & $9.32 \mathrm{e}-3$ & 2.245 & $4.047 \mathrm{e}-5$ & 0.8626 \\
\hline $4.64 \mathrm{e}-2$ & $1.95 \mathrm{e}-2$ & 2.169 & $1.878 \mathrm{e}-4$ & 0.8620 \\
\hline 0.1 & $2.84 \mathrm{e}-2$ & 2.148 & $4.047 \mathrm{e}-4$ & 0.8617 \\
\hline 0.215 & $4.15 \mathrm{e}-2$ & 2.135 & $8.719 \mathrm{e}-4$ & 0.8614 \\
\hline 0.464 & $6.07 \mathrm{e}-2$ & 2.125 & $1.878 \mathrm{e}-3$ & 0.8610 \\
\hline 1.0 & $8.89 \mathrm{e}-2$ & 2.118 & $4.047 \mathrm{e}-3$ & 0.8607 \\
\hline 2.154 & $1.30 \mathrm{e}-1$ & 2.114 & $8.719 \mathrm{e}-3$ & 0.8604 \\
\hline 4.641 & $1.91 \mathrm{e}-1$ & 2.111 & $1.879 \mathrm{e}-2$ & 0.8601 \\
\hline 10.0 & $2.80 \mathrm{e}-1$ & 2.109 & $4.047 \mathrm{e}-2$ & 0.8598 \\
\hline
\end{tabular}

Table 6.2 P.u.1. parameters of Example 6.3 


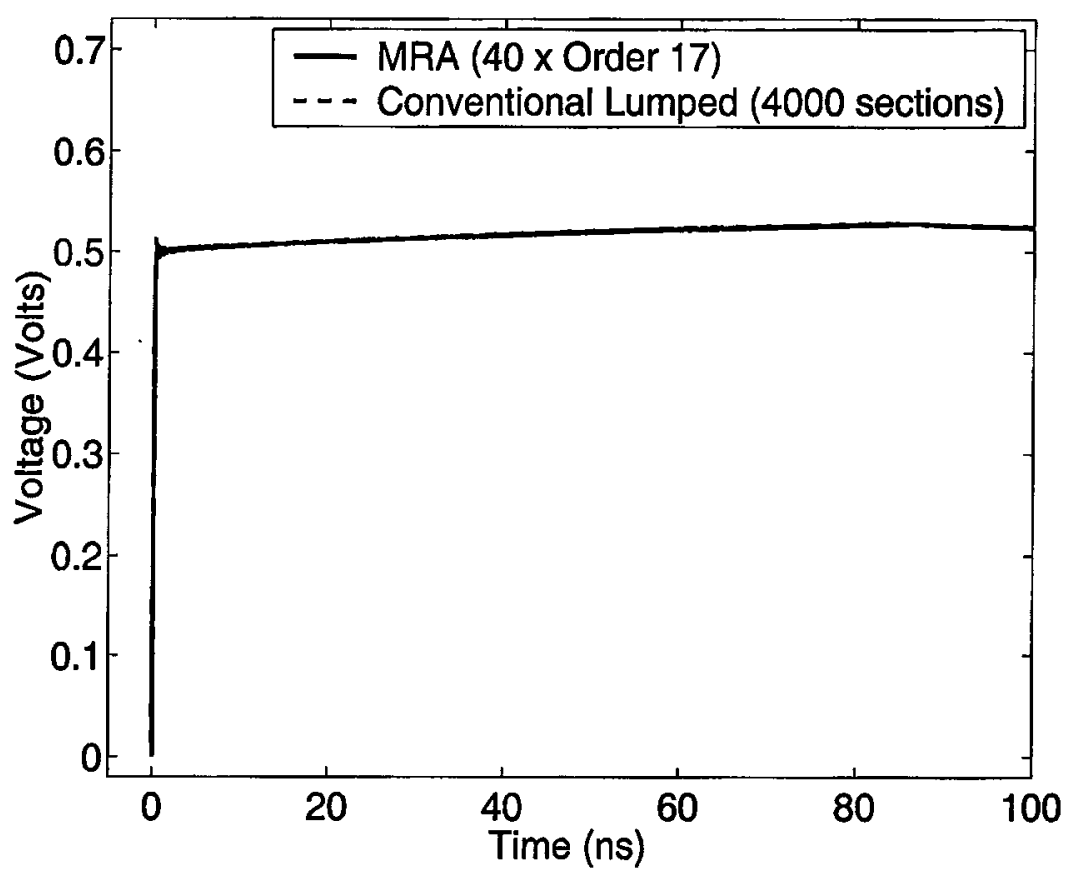

a) At node V1 (near end)

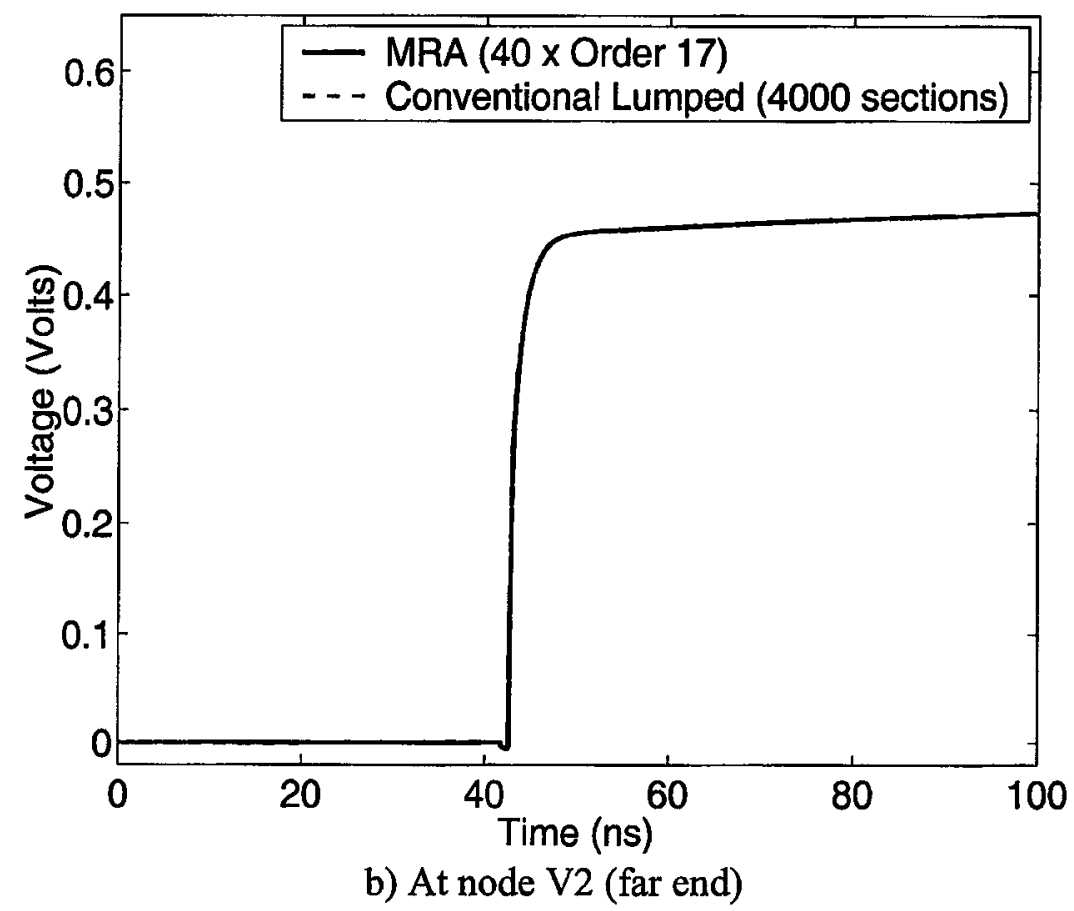

Figure 6.13 Time Domain Response (Example 6.3) 


\section{Chapter 7}

\section{Passive Model Reduction of Distributed Transmission Line Networks}

\subsection{Introduction}

When dealing with large linear networks composed of many lumped and distributed elements, model-order-reduction (MOR) techniques can be used to obtain efficient macromodels. This chapter describes a new technique to advance Krylov-space based passive model reduction algorithms to include distributed transmission lines described by Telegrapher's equations [54]-[56], [96]. Transmission lines can be lossy, coupled and can include frequency-dependent parameters. In the proposed scheme, transmission line subnetworks are treated with closed-form stamps obtained using the matrix rational approximation (MRA) algorithm, where the coefficients describing the macromodel are computed a priori and analytically. In addition, the method guarantees that the contribution of the MRA stamp to the modified nodal analysis (MNA) formulation leads to passive reduced-order macromodels.

The organization of this chapter is as follows. The formulation of the network equations is presented in section 7.2. Section 7.3 describes the proposed reduction algorithm and the passivity preservation proof. Numerical examples showing the validity of the proposed approach are shown in section 7.4. 


\subsection{Formulation of Network Equations}

As described in Chapter 2, a distributed linear network can be expressed as

$$
\begin{gathered}
C_{\phi} \dot{x}_{\phi}(t)+G_{\phi} x_{\phi}(t)+D i(t)=b_{\phi}(t) \\
I(s)=Y(s) V(s)
\end{gathered}
$$

where the matrices of (7.1) are defined in section 2.4.3. For clarity, and without loss of generality, (7.1) contains one transmission line network. The distributed network of (7.1) does not have a direct representation in the time-domain, which makes it difficult to include with nonlinear circuit simulators. In the previous three chapters, the MRA algorithm is used to convert $(7.1)$ to

$$
C_{\pi} \frac{d}{d t} \boldsymbol{x}_{\pi}(t)+G_{\pi} \boldsymbol{x}_{\pi}(t)=\boldsymbol{b}_{\pi}(t)
$$

where the distributed elements are now explicitly embedded in the MNA matrices of the overall network and

$$
G_{\pi}=G_{a}+G_{T} \quad C_{\pi}=C_{a}+C_{T}
$$

Here the matrices $G_{a}, C_{a}, x_{\pi}(t)$ and $b_{\pi}(t)$ are obtained from $\boldsymbol{G}_{\phi}, C_{\phi}, \boldsymbol{x}_{\phi}(t)$ and $\boldsymbol{b}_{\phi}(t)$ by appending them by rows and/or columns that contain zeros to account for the extra variables required for the stamp of the transmission line. Thus, $\boldsymbol{G}_{\boldsymbol{a}}, \boldsymbol{C}_{\boldsymbol{a}}, \boldsymbol{x}_{\pi}(t)$ and $\boldsymbol{b}_{\pi}(t)$ can be expressed in the following block form

$$
\boldsymbol{G}_{a}=\left[\begin{array}{cc}
G_{\phi} & 0 \\
0 & 0
\end{array}\right] \quad C_{a}=\left[\begin{array}{cc}
C_{\phi} & 0 \\
0 & 0
\end{array}\right] \quad x_{\pi}(t)=\left[\begin{array}{c}
x_{\phi}(t) \\
x_{T}(t)
\end{array}\right] \quad b_{\pi}(t)=\left[\begin{array}{c}
b_{\phi}(t) \\
0
\end{array}\right]
$$

where $\boldsymbol{x}_{T}(t)$ are the variables introduced by the transmission line macromodel. The matrices $\boldsymbol{G}_{T}$ and $\boldsymbol{C}_{T}$ correspond to the stamp of the transmission line. For the circuit realization described in Chapter 4, the matrices $G_{T}$ and $C_{T}$ can be expressed as

$$
G_{T}=\sum_{i}\left(\psi_{i}\right)^{T} G_{i} \psi_{i} \quad C_{T}=\sum_{i}\left(\psi_{i}\right)^{T} C_{i} \Psi_{i}
$$

where $\psi_{i}$ is a selector matrix that maps the block matrices $G_{i}$ and $C_{i}$ to the rest of the network equations and the superscript $T$ denotes the transpose of the matrix. The ma- 
trices $G_{i}$ and $C_{i}$ are described in detail in Chapter 4 for the RC, lossless and lossy (RLGC) transmission line models. Substituting (7.5) into (7.3), gives

$$
\boldsymbol{G}_{\pi}=G_{a}+\sum_{i}\left(\psi_{i}\right)^{T} G_{i} \psi_{i} \quad C_{\pi}=C_{a}+\sum_{i}\left(\psi_{i}\right)^{T} C_{i} \psi_{i}
$$

For clarity, and without loss of generality the discussion will focus on lossy frequencyindependent parameter case. For the case of RC, lossless and frequency dependent parameter transmission lines similar arguments can be used to prove the passivity of the reduced-order system. The $G_{i}$ and $C_{i}$ matrices for lossy transmission lines are described by (4.26) and (4.27), as

$$
\begin{aligned}
& \boldsymbol{G}_{i}=\left[\begin{array}{ccccccc}
\left(\frac{x_{i}}{d}+\frac{x_{i}^{2}+y_{i}^{2}}{4 x_{i} d}\right) R^{-1}+\frac{d}{4 x_{i}} G & \frac{-x_{i}}{d} R^{-1} & 0 & \frac{-d}{4 x_{i}} G & \frac{-\left(x_{i}^{2}+y_{i}^{2}\right)}{4 x_{i} d} R^{-1} & 0 & 0 \\
\frac{-x_{i}}{d} R^{-1} & \frac{x_{i}}{d} R^{-1} & 0 & 0 & 0 & U & 0 \\
0 & 0 & \frac{x_{i} d}{x_{i}^{2}+y_{i}^{2}} G & \frac{x_{i} d}{x_{i}^{2}+y_{i}^{2}} G & 0 & -U & 0 \\
\frac{-d}{4 x_{i}} G & 0 & \frac{x_{i} d}{x_{i}^{2}+y_{i}^{2}} G\left(\frac{x_{i} d}{x_{i}^{2}+y_{i}^{2}}+\frac{d}{4 x_{i}}\right) G & 0 & 0 & U \\
\frac{-\left(x_{i}^{2}+y_{i}^{2}\right)}{4 x_{i} d} R^{-1} & 0 & 0 & 0 & \frac{x_{i}^{2}+y_{i}^{2}}{4 x_{i} d} R^{-1} & 0 & -U \\
0 & -U & U & 0 & 0 & 0 & 0 \\
0 & 0 & 0 & -U & U & 0 & 0
\end{array}\right] \\
& C_{i}=\left[\begin{array}{ccccccc}
\frac{d}{4 x_{i}} C & 0 & 0 & \frac{-d}{4 x_{i}} C & 0 & 0 & 0 \\
0 & 0 & 0 & 0 & 0 & 0 & 0 \\
0 & 0 & \frac{x_{i} d}{x_{i}^{2}+y_{i}^{2}} C & \frac{x_{i} d}{x_{i}^{2}+y_{i}^{2}} C & 0 & 0 & 0 \\
\frac{d}{4 x_{i}} C & 0 & \frac{x_{i} d}{x_{i}^{2}+y_{i}^{2}} C\left(\frac{x_{i} d}{x_{i}^{2}+y_{i}^{2}}+\frac{d}{4 x_{i}}\right) & C & 0 & 0 & 0 \\
0 & 0 & 0 & 0 & 0 & 0 & 0 \\
0 & 0 & 0 & 0 & 0 & \frac{d}{x_{i}} L & 0 \\
0 & 0 & 0 & 0 & 0 & 0 & \frac{4 x_{i} d}{x_{i}^{2}+y_{i}^{2}} L
\end{array}\right]
\end{aligned}
$$

for the subsections described by (4.24), for $i>0$, and 


$$
\boldsymbol{G}_{0}=\left[\begin{array}{cccc}
\frac{a_{0}}{2 d} R^{-1}+\frac{d}{2 a_{0}} \boldsymbol{G} & \frac{-a_{0}}{2 d} R^{-1} & \frac{d}{2 a_{0}} \boldsymbol{G} & \mathbf{0} \\
\frac{-a_{0}}{2 d} R^{-1} & \frac{a_{0}}{2 d} R^{-1} & 0 & \boldsymbol{U} \\
\frac{d}{2 a_{0}} \boldsymbol{G} & 0 & \frac{d}{2 a_{0}} \boldsymbol{G} & -\boldsymbol{U} \\
0 & -U & \boldsymbol{U} & 0
\end{array}\right] \quad C_{0}=\left[\begin{array}{cccc}
\frac{d}{2 a_{0}} \boldsymbol{C} & \mathbf{0} & \frac{d}{2 a_{0}} \boldsymbol{C} & 0 \\
0 & 0 & 0 & 0 \\
\frac{d}{2 a_{0}} \boldsymbol{C} & 0 & \frac{d}{2 a_{0}} \boldsymbol{C} & 0 \\
0 & 0 & 0 & \frac{2 d}{a_{0}} L
\end{array}\right]
$$

for the subsection described by (4.25). It should be noted that the predetermined coefficients $x_{i}, x_{i}^{2}+y_{i}^{2}$ and $a_{0}$ are all positive values due to the Hurwitz condition imposed by Theorem 3.1.

The linear multi-port network of (7.2) can be expressed in the frequency-domain as

$$
\begin{gathered}
\left(s C_{\pi}+G_{\pi}\right) \boldsymbol{X}_{\pi}=\boldsymbol{B}_{\pi} \boldsymbol{U}_{p} \\
I_{p}=\boldsymbol{B}_{\pi}^{T} \boldsymbol{X}_{\pi}
\end{gathered}
$$

where

- $\boldsymbol{U}_{p}$ and $\boldsymbol{I}_{p}$ are the port voltages and currents, respectively.

- $\boldsymbol{B}_{\pi}=\left[b_{i, j} \in\{0,-1\}\right]$ is a selector matrix that maps the port voltages to network, where $i \in\{1, \ldots, \pi\}, j \in\{1, \ldots, p\}, \pi$ is the total number of variables in the MNA formulation and $p$ is the number of ports.

Solving for the port current variables, the Y-parameters of (7.9) become

$$
\boldsymbol{Y}(s)=\boldsymbol{B}_{\pi}^{T}\left(s C_{\pi}+\boldsymbol{G}_{\pi}\right)^{-1} \boldsymbol{B}_{\pi}
$$

In the following section, a passive MOR algorithm is described.

\subsection{Model Reduction and Passivity Preservation}

This section describes a passive reduction algorithm for distributed transmission lines based on congruent transformation. The proposed algorithm uses the MRA macromodel to describe transmission lines in the time-domain. In addition, a proof of passivity for the reduced-order macromodel is presented. 


\subsubsection{Model Reduction}

In Chapter 2, section 2.6.2, a MOR algorithm is described based on Krylov subspace techniques. The first step of the algorithm is to construct an orthonormal matrix $\boldsymbol{Q}$ using the block Arnoldi algorithm, such that [13],

$$
\begin{gathered}
\operatorname{colsp}(\boldsymbol{Q})=\operatorname{Kr}\left(\boldsymbol{A}_{\pi}, \boldsymbol{R}_{\pi}, k\right) \\
\boldsymbol{Q}^{\mathrm{T}} \boldsymbol{Q}=\boldsymbol{I}_{k}
\end{gathered}
$$

where

$$
\begin{array}{cc}
\boldsymbol{A}_{\pi}=-\boldsymbol{G}_{\pi}^{-1} \boldsymbol{C}_{\pi} & \boldsymbol{R}_{\pi}=\boldsymbol{G}_{\pi}^{-1} \boldsymbol{B}_{\pi} \\
\operatorname{Kr}\left(\boldsymbol{A}_{\pi}, \boldsymbol{R}_{\pi}, q\right)=\operatorname{colsp}\left[\boldsymbol{R}_{\pi}, A_{\pi} \boldsymbol{R}_{\pi}, A_{\pi}^{2} \boldsymbol{R}, \ldots, A_{\pi}^{q-1} \boldsymbol{R}_{\pi}\right]
\end{array}
$$

and $\boldsymbol{I}_{k} \in \mathfrak{R}^{k \times k}$ is the identity matrix. The variable $k$ corresponds to the size of the reduced-order network defined as $k=q \cdot p$, where $p$ is the number of ports. Next, the matrix $\boldsymbol{Q}$ is used to reduce the augmented system matrices of (7.10) using the congruence transform as

$$
\tilde{\boldsymbol{G}}_{k}=\boldsymbol{Q}^{\mathrm{T}} \boldsymbol{G}_{\pi} \boldsymbol{Q} \quad \tilde{\boldsymbol{C}}_{k}=\boldsymbol{Q}^{\mathrm{T}} \boldsymbol{C}_{\pi} \boldsymbol{Q} \quad \tilde{\boldsymbol{B}}_{k}=\boldsymbol{Q}^{\mathrm{T}} \boldsymbol{B}_{\pi}
$$

The admittance matrix for the reduced system is given by

$$
\tilde{\boldsymbol{Y}}(s)=\tilde{\boldsymbol{B}}_{k}^{T}\left(s \tilde{\boldsymbol{C}}_{\boldsymbol{k}}+\tilde{\boldsymbol{G}}_{k}\right)^{-1} \tilde{\boldsymbol{B}}_{k}
$$

It can be shown that the reduced-order system described by (7.14) and (7.15) preserves the first $k$ block moments. The proof of the preservation of moments is identical to the one given in [13], where the matrices $\boldsymbol{C}_{\boldsymbol{\pi}}$ and $\boldsymbol{G}_{\boldsymbol{\pi}}$ contain stamps of lumped components only. However, a new approach is needed to prove that the reduced system is passive since the matrices $C_{\pi}$ and $G_{\pi}$ of the original system contain the stamps of the MRA macromodel (7.6)-(7.7).

\subsubsection{Passivity Preservation}

In this section, a proof is provided demonstrating that the reduced-order model of (7.15) is passive. As mentioned in Chapter 3, a linear network with an admittance matrix $Y(s)$ is 
said to be passive for all signals of $z$, if and only if [77]-[79],

1. $Y\left(s^{*}\right)=Y^{*}(s)$ where ${ }^{*}$ is the complex conjugate operator.

2. $Y(s)$ is a positive real matrix. That is the product $z^{* T}\left[Y^{T}\left(s^{*}\right)+Y(s)\right] z \geq 0$ for all possible values of $s$ satisfying $\operatorname{Re}(s)>0$ and any arbitrary value of $z$.

The first condition is automatically satisfied since the reduced matrices, $\tilde{C}_{\pi}, \tilde{G}_{\pi}$ and $\tilde{\boldsymbol{B}}_{\boldsymbol{\pi}}$ are all real. To show that the second condition is also satisfied, the Y-parameters of the reduced system (7.15) is used to construct

$$
z^{* T} \tilde{Y}_{h}(s) z=z^{* T}\left[\tilde{\boldsymbol{Y}}^{T}\left(s^{*}\right)+\tilde{\boldsymbol{Y}}(s)\right] z
$$

Inserting (7.15) into (7.16) and using some vector algebra gives

$$
\begin{aligned}
\boldsymbol{z}^{* \mathbf{T}} \tilde{\boldsymbol{Y}}_{\boldsymbol{h}}(s) \boldsymbol{z} & =\boldsymbol{z}^{* \mathbf{T}} \tilde{\boldsymbol{B}}_{k}{ }^{T}\left[\left(\tilde{\boldsymbol{G}}_{k+S} \tilde{\boldsymbol{C}}_{k}\right)^{-1}+\left(\left(\tilde{\boldsymbol{G}}_{k+S}{ }^{*} \tilde{\boldsymbol{C}}_{k}\right)^{-1}\right)^{T}\right] \tilde{\boldsymbol{B}}_{k} \boldsymbol{z} \\
& =\boldsymbol{z}^{*}{ }^{\mathrm{T}} \tilde{\boldsymbol{B}}_{k}{ }^{T}\left(\tilde{\boldsymbol{G}}_{k+S} \tilde{\boldsymbol{C}}_{k}\right)^{-1}\left[\left(\tilde{\boldsymbol{G}}_{k+S} \tilde{\boldsymbol{C}}_{k}\right)+\left(\tilde{\boldsymbol{G}}_{k+S}{ }^{*} \tilde{\boldsymbol{C}}_{k}\right)^{T}\right]\left(\left(\tilde{\boldsymbol{G}}_{k+S}{ }^{*} \tilde{\boldsymbol{C}}_{k}\right)^{-1}\right)^{T} \tilde{\boldsymbol{B}}_{k}{ }^{(7}
\end{aligned}
$$

Setting $\left.\Phi=\left(\tilde{\boldsymbol{G}}_{k}+s^{*} \tilde{\boldsymbol{C}}_{k}\right)^{-1}\right)^{T} \tilde{\boldsymbol{B}}_{k} \boldsymbol{z}, s=\sigma+j \omega$ and using (7.14) yields

$$
\begin{aligned}
z^{* T} \tilde{Y}_{h}(s) z & =\Phi^{* T}\left[\tilde{G}_{k}+\tilde{G}_{k}{ }^{T}+\sigma\left(\tilde{C}_{k}+\tilde{C}_{k}{ }^{T}\right)\right] \Phi \\
& =\Phi^{* T} Q^{T}\left[G_{\pi}+G_{\pi}{ }^{T}+\sigma\left(C_{\pi}+C_{\pi}{ }^{T}\right)\right] Q \Phi \\
& =y^{* T}\left[G_{\pi}+G_{\pi}{ }^{T}\right] y+y^{* T}\left[\sigma\left(C_{\pi}+C_{\pi}{ }^{T}\right)\right] y
\end{aligned}
$$

where $y=Q \Phi$. Substituting (7.4) and (7.6) for the matrices $G_{\pi}$ and $C_{\pi}$ in (7.18) and using the fact that $C_{\phi}$ is symmetric yields

$$
\begin{aligned}
& z^{* T} \hat{Y}_{h}(s) z=y^{*}\left(\left[\begin{array}{cc}
G_{\phi}+G_{\phi}^{T} & 0 \\
0 & 0
\end{array}\right]\right) y+2 y^{* T} \sigma\left(\left[\begin{array}{cc}
C_{\phi} & 0 \\
0 & 0
\end{array}\right]\right) y \\
& +y^{* T}\left(\sum_{i}\left(\psi_{i}\right)^{T}\left(G_{i}+\left(G_{i}\right)^{T}\right) \psi_{i}\right) y+y^{*^{T}} \sigma\left(\sum_{i}\left(\psi_{i}\right)^{T}\left(C_{i}+\left(C_{i}\right)^{T}\right) \psi_{i}\right) y
\end{aligned}
$$


To prove that the second condition is satisfied, each one of the four quadratic forms in (7.19) is shown to be nonnegative definite. The matrices $C_{\phi}$ and $G_{\phi}+G_{\phi}^{\mathrm{T}}$ describe the MNA equation of lumped elements and can be formulated to be nonnegative definite [13], hence,

$$
y^{* T}\left(\left[\begin{array}{cc}
G_{\phi}+G_{\phi}^{T} & 0 \\
0 & 0
\end{array}\right]\right) y \geq 0 \quad 2 y^{*^{T}} \sigma\left(\left[\begin{array}{cc}
C_{\phi} & 0 \\
0 & 0
\end{array}\right]\right) y \geq 0
$$

On the other hand, proving that the last two quadratic forms in (7.19) are nonnegative requires showing that matrices $\left(\Psi_{i}\right)^{T}\left(G_{i}+\left(G_{i}\right)^{T}\right) \Psi_{i}$ and $\left(\Psi_{i}\right)^{T}\left(C_{i}+\left(C_{i}\right)^{T}\right) \psi_{i}$ are nonnegative definite. These matrices are described in the form of a congruence transform of the matrices $\boldsymbol{G}_{i}+\left(\boldsymbol{G}_{i}\right)^{T}$ and $\boldsymbol{C}_{i}+\left(\boldsymbol{C}_{i}\right)^{T}$, respectively, where $\psi_{i}$ is used as the transformation matrix. Hence, $\left(\psi_{i}\right)^{T}\left(G_{i}+\left(G_{i}\right)^{T}\right) \psi_{i}$ and $\left(\psi_{i}\right)^{T}\left(C_{i}+\left(C_{i}\right)^{T}\right) \psi_{i}$ are nonnegative definite if $G_{i}+\left(G_{i}\right)^{T}$ and $C_{i}+\left(C_{i}\right)^{T}$ are nonnegative definite [97]. Thus to complete the proof of passivity it is necessary to show that $\boldsymbol{G}_{\boldsymbol{i}}+\left(\boldsymbol{G}_{\boldsymbol{i}}\right)^{T}$ and $\boldsymbol{C}_{\boldsymbol{i}}+\left(\boldsymbol{C}_{\boldsymbol{i}}\right)^{T}$ are nonnegative definite. This proof is given in the next subsection through establishing the basic properties of the matrices $\boldsymbol{G}_{\boldsymbol{i}}$ and $\boldsymbol{C}_{\boldsymbol{i}}$.

\section{Properties of $G_{i}$ and $C_{i}$ Matrices}

For ease of presentation, the $\boldsymbol{G}_{\boldsymbol{i}}$ and $\boldsymbol{C}_{\boldsymbol{i}}$ matrices of subsection (7.7) is considered, since similar arguments can be used to treat the subsection of (7.8). Using (7.7), $G_{i}$ and $C_{i}$ can be represented as,

$$
G_{i}=\left[\begin{array}{cc}
W_{i} & E \\
-E^{T} & 0
\end{array}\right] \quad C_{i}=\left[\begin{array}{cc}
Z_{i} & 0 \\
0 & H_{i}
\end{array}\right]
$$

where 


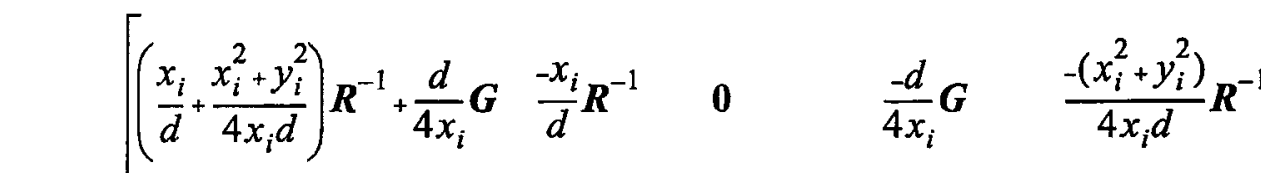

$$
\begin{aligned}
& W_{i}=\left[\begin{array}{ccccc}
\frac{-x_{i}}{d} R^{-1} & \frac{x_{i}}{d} R^{-1} & 0 & 0 & 0 \\
0 & 0 & \frac{x_{i} d}{x_{i}^{2}+y_{i}^{2}} G & \frac{x_{i} d}{x_{i}^{2}+y_{i}^{2}} G & 0 \\
\frac{-d}{4 x_{i}} G & 0 & \frac{x_{i} d}{x_{i}^{2}+y_{i}^{2}} G\left(\frac{x_{i} d}{x_{i}^{2}+y_{i}^{2}}+\frac{d}{4 x_{i}}\right) G & 0 \\
\frac{-\left(x_{i}^{2}+y_{i}^{2}\right)}{4 x_{i} d} R^{-1} & 0 & 0 & 0 & \frac{x_{i}^{2}+y_{i}^{2}}{4 x_{i} d} R^{-1}
\end{array}\right] \\
& Z_{i}=\left[\begin{array}{ccccc}
\frac{d}{4 x_{i}} C & 0 & 0 & \frac{-d}{4 x_{i}} C & 0 \\
0 & 0 & 0 & 0 & 0 \\
0 & 0 & \frac{x_{i} d}{x_{i}^{2}+y_{i}^{2}} C & \frac{x_{i} d}{x_{i}^{2}+y_{i}^{2}} C & 0 \\
\frac{-d}{4 x_{i}} C & 0 & \frac{x_{i} d}{x_{i}^{2}+y_{i}^{2}} C & \left(\frac{x_{i} d}{x_{i}^{2}+y_{i}^{2}}+\frac{d}{4 x_{i}}\right) & C \\
0 & 0 & 0 & 0 & 0
\end{array}\right] \\
& \boldsymbol{E}^{\mathbf{T}}=\left[\begin{array}{ccccc}
\mathbf{0} & \boldsymbol{U} & -\boldsymbol{U} & \mathbf{0} & \mathbf{0} \\
\mathbf{0} & \mathbf{0} & \mathbf{0} & \boldsymbol{U} & -\boldsymbol{U}
\end{array}\right]
\end{aligned}
$$

Clearly $\boldsymbol{H}_{\boldsymbol{i}}$ is symmetric nonnegative definite since it is block diagonal with symmetric nonnegative definite diagonal blocks (i.e. the per-unit-length (p.u.1.) parameters are nonnegative definite and the predetermined coefficients are positive) [97]. The next two theorems are developed to show that $W_{i}$ and $Z_{i}$ are nonnegative definite.

\section{Theorem 7.1}

Let $\Theta$ be a block structured matrix that has only 4 nonzero block matrices located at the block entries $(i, i),(i, j),(j, i)$ and $(j, j)$. Assume that these four blocks are equal to $K$, i.e., 


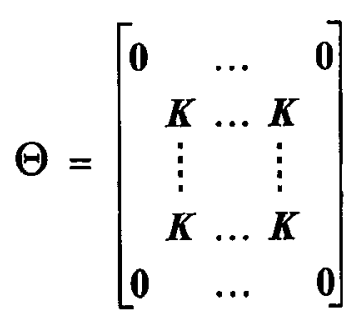

where $K \in \mathfrak{R}^{q \times q}$ is a nonnegative definite matrix. Then $\Theta$ is nonnegative definite.

\section{Proof of Theorem 7.1}

To simplify the proof considerably, consider a permutated version of $\Theta, \Theta_{P}$, where

$$
\Theta=P^{T} \Theta_{P} P
$$

and $\boldsymbol{P}$ is some suitable permutation matrix chosen such that,

$$
\Theta_{P}=\left[\begin{array}{ccccc}
K & K & 0 & \ldots & 0 \\
K & K & 0 & \ldots & 0 \\
0 & 0 & 0 & \ldots & 0 \\
\vdots & \vdots & \vdots & & \vdots \\
0 & 0 & 0 & \ldots & 0
\end{array}\right]
$$

$\Theta_{P}$ can be expressed in terms of congruence transform as

$$
\Theta_{P}=F^{T}\left[\begin{array}{cccc}
K & 0 & \ldots & 0 \\
0 & 0 & \ldots & 0 \\
\vdots & \vdots & & \vdots \\
0 & 0 & \ldots & 0
\end{array}\right] F
$$

where

$$
F=\left[\begin{array}{ccccc}
U & U & 0 & \ldots & 0 \\
0 & U & 0 & \ldots & 0 \\
0 & 0 & U & \ldots & 0 \\
\vdots & \vdots & \vdots & & \vdots \\
0 & 0 & 0 & \ldots & U
\end{array}\right]
$$

and $U$ is the identity matrix. Using (7.24)-(7.27), the matrix $\Theta$ can be written in the form of the congruence transform 


$$
\Theta=(F P)^{T}\left[\begin{array}{cccc}
K & 0 & \ldots & 0 \\
0 & 0 & \ldots & 0 \\
\vdots & \vdots & & \vdots \\
0 & 0 & \ldots & 0
\end{array}\right](F P)
$$

Since $K$ is nonnegative definite, then $\Theta$ is nonnegative definite since it is defined as a congruence transform using $\boldsymbol{F P}$ as the transformation operator [97].

\section{Theorem 7.2}

Let $\Psi$ be a matrix that has the same structure as $\Theta$ except that the blocks at the entries $(i, j)$ and $(j, i)$ are negated, i.e.,

$$
\Psi=\left[\begin{array}{ccccc}
0 & & \ldots & & 0 \\
& K & \ldots & -K \\
& \vdots & & \vdots & \\
& -K & \ldots & K & \\
0 & & \ldots & & 0
\end{array}\right]
$$

where $K \in \mathfrak{R}^{q \times q}$ is a nonnegative definite matrix. Then $\Psi$ is nonnegative definite.

\section{Proof of Theorem 7.2}

The proof of this theorem is similar to the above theorem where in this case a permutated matrix $\Psi_{P}$ is used to put $\Psi$ in the form of the congruence transform as

$$
\Psi=P^{T} \Psi_{P} P
$$

where $\boldsymbol{P}$ is some suitable permutation matrix such that

$$
\Psi_{P}=\left[\begin{array}{ccccc}
K & -K & 0 & \ldots & 0 \\
-K & K & 0 & \ldots & 0 \\
0 & 0 & 0 & \ldots & 0 \\
\vdots & \vdots & \vdots & & \vdots \\
0 & 0 & 0 & \ldots & 0
\end{array}\right]
$$

$\Psi_{P}$ can be expressed in the form 


$$
\Psi_{P}=F^{T}\left[\begin{array}{cccc}
K & 0 & \ldots & 0 \\
0 & 0 & \ldots & 0 \\
\vdots & \vdots & & \vdots \\
0 & 0 & \ldots & 0
\end{array}\right] F
$$

where

$$
\boldsymbol{F}=\left[\begin{array}{ccccc}
\boldsymbol{U} & -\boldsymbol{U} & \mathbf{0} & \ldots & 0 \\
\mathbf{0} & \boldsymbol{U} & \mathbf{0} & \ldots & 0 \\
\mathbf{0} & 0 & \boldsymbol{U} & \ldots & 0 \\
\vdots & \vdots & \vdots & & \vdots \\
0 & 0 & 0 & \ldots & U
\end{array}\right]
$$

Using (7.30)-(7.33), $\Psi$ can be written in terms of congruence transform

$$
\Psi=(F P)^{T}\left[\begin{array}{cccc}
K & 0 & \ldots & 0 \\
0 & 0 & \ldots & 0 \\
\vdots & \vdots & & \vdots \\
0 & 0 & \ldots & 0
\end{array}\right](F P)
$$

Since $K$ is nonnegative definite, then $\Psi$ is nonnegative definite since it is defined as congruence transform using $F P$ and as the transformation operator.

\section{Corollary 7.1}

The matrices $W_{i}$ and $Z_{i}$ in (7.22) are symmetric nonnegative definite.

\section{Proof of Corollary 7.1}

The matrices $W_{i}$ and $Z_{i}$ can be written as

$$
W_{i}=W_{i, 1}+W_{i, 2}+W_{i, 3}+W_{i, 4} \quad Z_{i}=Z_{i, 1}+Z_{i, i}
$$

where 


$$
\begin{aligned}
& W_{i, 1}=\left[\begin{array}{ccccc}
\frac{x_{i}^{2}+y_{i}^{2}}{4 x_{i} d} R^{-1} & 0 & 0 & 0 & \frac{-\left(x_{i}^{2}+y_{i}^{2}\right)}{4 x_{i} d} R^{-1} \\
0 & 0 & 0 & 0 & 0 \\
0 & 0 & 0 & 0 & 0 \\
0 & 0 & 0 & 0 & 0 \\
\frac{-\left(x_{i}^{2}+y_{i}^{2}\right)}{4 x_{i} d} R^{-1} & 0 & 0 & 0 & \frac{x_{i}^{2}+y_{i}^{2}}{4 x_{i} d} R^{-1}
\end{array}\right] \\
& W_{i, 2}=\left[\begin{array}{ccccc}
\frac{x_{i}}{d} R^{-1} & \frac{-x_{i}}{d} R^{-1} & 0 & 0 & 0 \\
\frac{-x_{i}}{d} R^{-1} & \frac{x_{i}}{d} R^{-1} & 0 & 0 & 0 \\
0 & 0 & 0 & 0 & 0 \\
0 & 0 & 0 & 0 & 0 \\
0 & 0 & 0 & 0 & 0
\end{array}\right] \\
& W_{i, 3}=\left[\begin{array}{ccccc}
\frac{d}{4 x_{i}} G & 0 & 0 & \frac{-d}{4 x_{i}} G & 0 \\
0 & 0 & 0 & 0 & 0 \\
0 & 0 & 0 & 0 & 0 \\
\frac{-d}{4 x_{i}} G & 0 & 0 & \frac{d}{4 x_{i}} G & 0 \\
0 & 0 & 0 & 0 & 0
\end{array}\right] \\
& W_{i, 4}=\left[\begin{array}{ccccc}
0 & 0 & 0 & 0 & 0 \\
0 & 0 & 0 & 0 & 0 \\
0 & 0 & \frac{x_{i} d}{x_{i}^{2}+y_{i}^{2}} G & \frac{x_{i} d}{x_{i}^{2}+y_{i}^{2}} G & 0 \\
0 & 0 & \frac{x_{i} d}{x_{i}^{2}+y_{i}^{2}} G & \frac{x_{i} d}{x_{i}^{2}+y_{i}^{2}} & 0 \\
0 & 0 & 0 & 0 & 0
\end{array}\right]
\end{aligned}
$$




$$
\begin{gathered}
Z_{i, 1}=\left[\begin{array}{ccccc}
\frac{d}{4 x_{i}} C & 0 & 0 & \frac{-d}{4 x_{i}} C & 0 \\
0 & 0 & 0 & 0 & 0 \\
0 & 0 & 0 & 0 & 0 \\
\frac{-d}{4 x_{i}} C & 0 & 0 & \frac{d}{4 x_{i}} C & 0 \\
0 & 0 & 0 & 0 & 0
\end{array}\right] \\
Z_{i, 2}=\left[\begin{array}{cccccc}
0 & 0 & 0 & 0 & 0 \\
0 & 0 & 0 & 0 & 0 \\
0 & 0 & \frac{x_{i} d}{x_{i}^{2}+y_{i}^{2}} C & \frac{x_{i} d}{x_{i}^{2}+y_{i}^{2}} C & 0 \\
0 & 0 & \frac{x_{i} d}{x_{i}^{2}+y_{i}^{2}} C & \frac{x_{i} d}{x_{i}^{2}+y_{i}^{2}} C & 0 \\
0 & 0 & 0 & 0 & 0
\end{array}\right]
\end{gathered}
$$

Due to Hurwitz condition of the scalar approximation of $e^{x}$ imposed by theorem 3.1, the constants $x_{i}^{2}+y_{i}^{2}$ and $x_{i}$ are all positive values. In addition, the p.u.l. parameter matrices $\boldsymbol{R}, \boldsymbol{L}, \boldsymbol{G}$ and $\boldsymbol{C}$ are nonnegative definite [2], [23]. Using theorems 7.1 and 7.2, all the block matrices in (7.36)-(7.41) are nonnegative definite. This implies that $W_{i}$ and $Z_{i}$ are the summation of symmetric nonnegative definite matrices, and hence $W_{i}$ and $Z_{i}$ are symmetric nonnegative definite [97].

\section{Corollary 7.2}

The matrices $C_{i}+\left(C_{i}\right)^{T}$ and $G_{i}+\left(G_{i}\right)^{T}$ are symmetric nonnegative definite.

\section{Proof of Corollary 7.2}

This result can be easily deduced by noting that

$$
C_{i}+\left(C_{i}\right)^{T}=\left[\begin{array}{cc}
Z_{i} & 0 \\
0 & H_{i}
\end{array}\right]+\left[\begin{array}{cc}
Z_{i} & 0 \\
0 & H_{i}
\end{array}\right]^{T}=\left[\begin{array}{cc}
2 Z_{i} & 0 \\
0 & 2 H_{i}
\end{array}\right]
$$




$$
G_{i}+\left(G_{i}\right)^{T}=\left[\begin{array}{cc}
W_{i} & E \\
-E^{\mathrm{T}} & 0
\end{array}\right]+\left[\begin{array}{cc}
W_{i} & E \\
-E^{\mathrm{T}} & 0
\end{array}\right]^{T}=\left[\begin{array}{cc}
2 W_{i} & 0 \\
0 & 0
\end{array}\right]
$$

which means that $C_{i}+\left(C_{i}\right)^{T}$ and $G_{i}+\left(G_{i}\right)^{T}$ are block diagonals with symmetric nonnegative definite diagonal blocks.

This concludes the proof of passivity for the reduced-order system. If the time-domain realization of Chapter 7 is used, then the reduced system will also be passive since the MNA equations satisfy (7.21) and the matrices $W_{i}$ and $Z_{i}$ of (7.35) can be written as summations of nonnegative matrices as described by (7.23) and (7.29). Next, a brief outline is provided to extend the proposed passivity preservation algorithm for reduction of transmission lines with frequency-dependent parameters.

\subsubsection{Transmission Lines with Frequency-Dependent Parameters}

In order to preserve the passivity of the reduced-order macromodel, the functions used to model the frequency-dependent parameters must be positive real. A technique to realize the frequency-dependent parameters in terms of positive-real functions is described in Chapter 5. The method matches the frequency response of the p.u.1. parameters in terms passive circuit elements composed of positive resistors, capacitors, inductors and ideal transformers. The matrices $G_{i}$ and $C_{i}$ including transmission lines with frequency-dependent parameters can also be represented in a form similar to (7.21). Hence the matrices $\boldsymbol{G}_{i}$ and $C_{i}$ for the case of frequency-dependent parameters can also be shown to be nonnegative definite using similar proof to the one presented in section 7.3.2.

\subsection{Examples}

Two examples are presented in this section to demonstrate the validity and efficiency of the proposed method. The second example includes interconnects with frequency-dependent parameters. The results given by the proposed method are compared with SPICE analysis. Within the context of this section, SPICE analysis refers to solving the transmis- 
sion line equations to obtain the network's frequency response or using the conventional lumped segmentation model [2] to obtain the network's time-domain response.

\subsubsection{Example 7.1}

A two-port linear subnetwork consisting of 1516 linear components (including 30 transmission lines) with nonlinear terminations is considered (Figure 7.1). The MRA macromodel (Padé order 5) was used for the transmission lines. The original set of MNA equations contained a total of 1682 variables. Using a multi-point version of the reduction algorithm of section 7.3 [12], the size of the reduced system obtained was $66 \times 66$. Figure 7.2 show a comparison of Y-parameters of the linear subnetwork, which are obtained using SPICE analysis and the proposed method. Figure 7.3 compares the time responses at two output nodes of the circuit (Vp2 and Vout). The input pulse used for this example has a rise/fall time of $0.1 \mathrm{~ns}$ and pulse width of $5 \mathrm{~ns}$. The transient simulation of the reducedorder system on a Sun Ultra 20 machine required 30 seconds of CPU time while the conventional lumped system required 817 seconds on the same machine (Table 7.1).

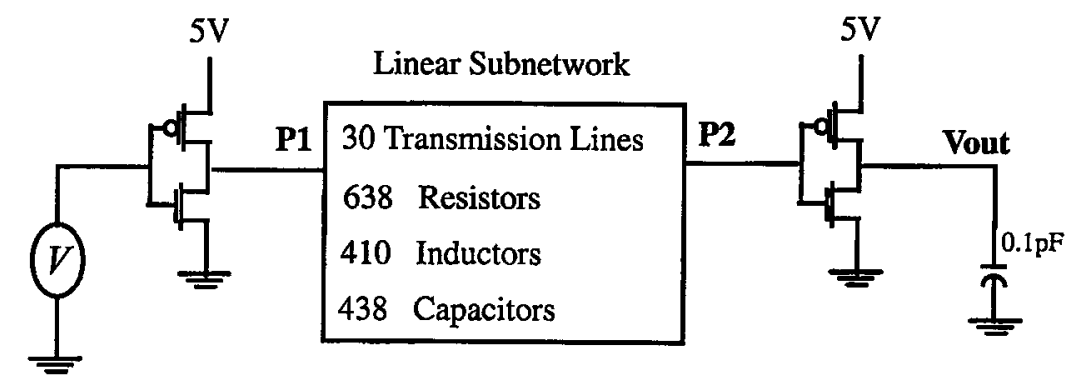

Figure 7.1 Linear subnetwork circuit with nonlinear termination (Example 7.1)

\begin{tabular}{|c|c|c|}
\hline $\begin{array}{c}\text { Proposed Method } \\
\text { (seconds) }\end{array}$ & $\begin{array}{c}\text { Conventional lumped model } \\
\text { (seconds) }\end{array}$ & Speed-up \\
\hline 30 & 817 & 27 \\
\hline
\end{tabular}

Table 7.1 CPU comparison of transient response (Example 7.1) 


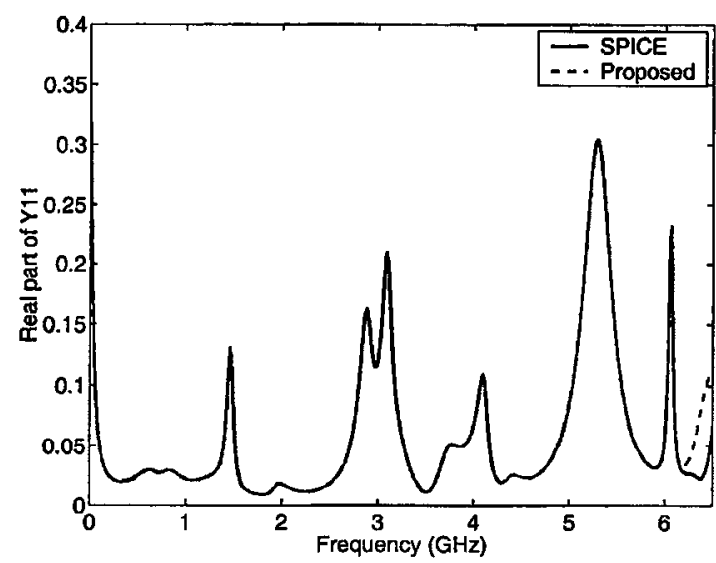

a) Real part of $Y 11$

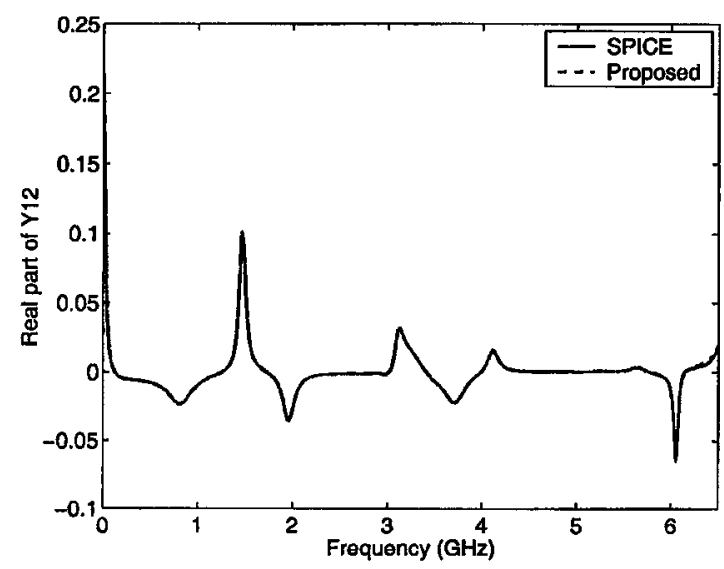

c) Real part of Y12

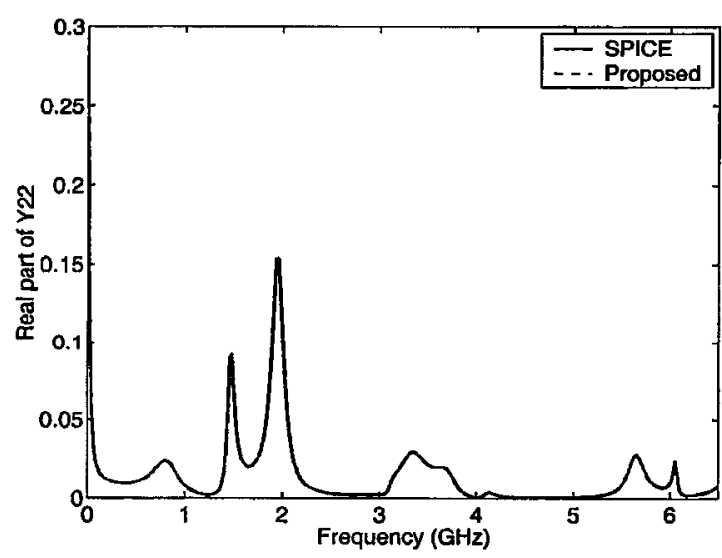

e) Real part of Y22

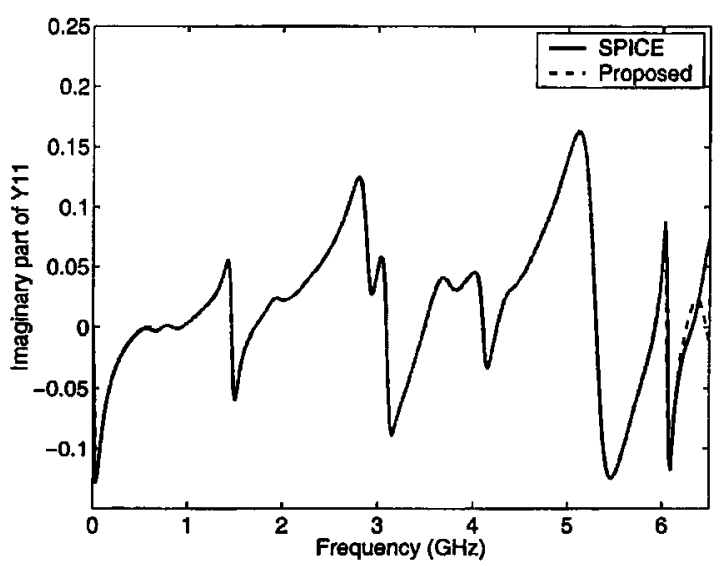

b) Imaginary part of Y11

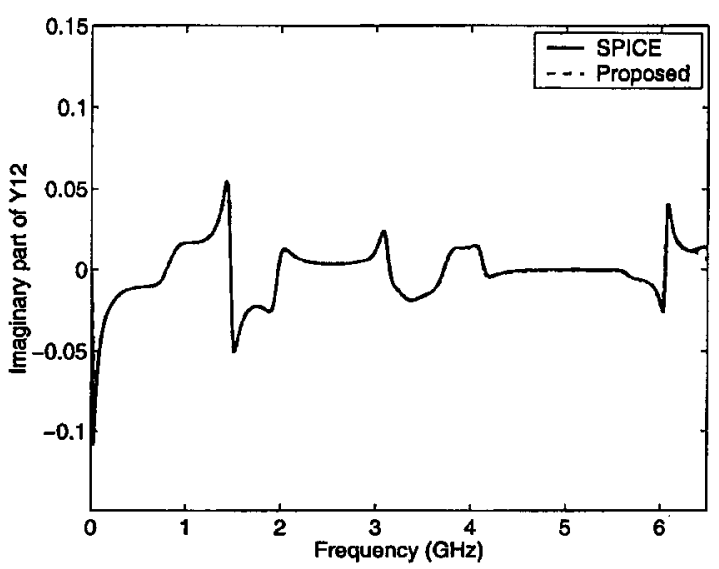

d) Imaginary part of Y12

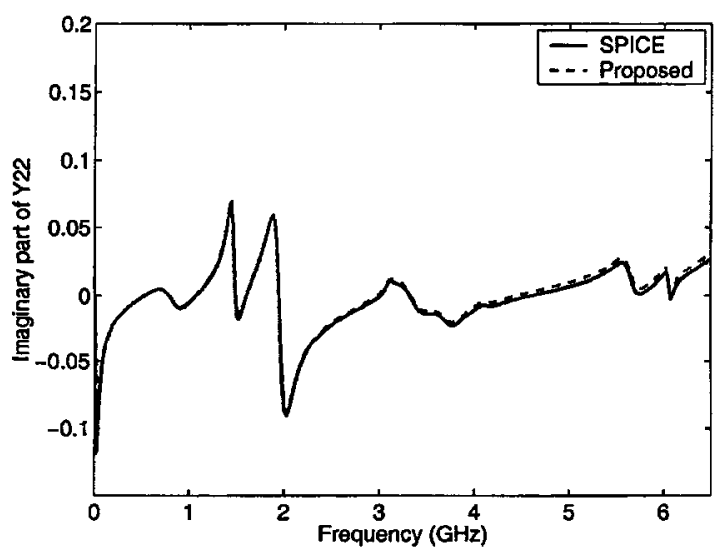

f) Imaginary part of Y22

Figure 7.2 Y-parameters of linear subnetwork (Example 7.1) 


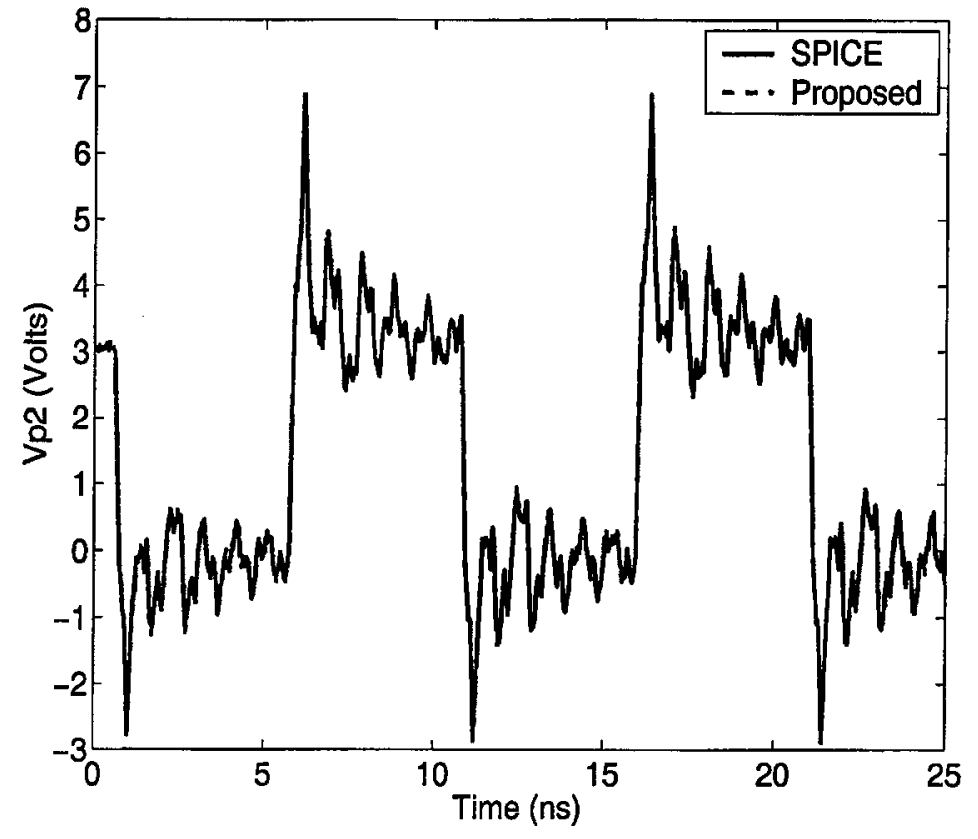

a) At port Vp2

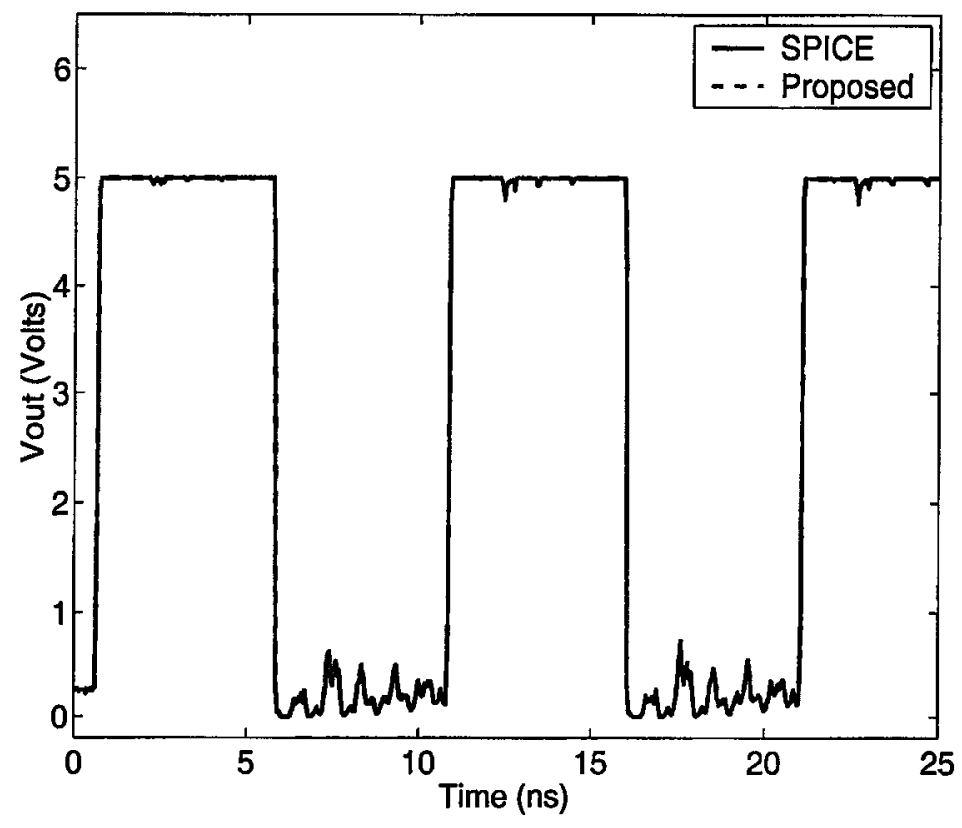

b) At node Vout

Figure 7.3 Transient Response (Example 7.1) 


\subsubsection{Example 7.2}

A two-port linear subnetwork consisting of 2203 linear components (including 35 interconnects with frequency-dependent parameters) with nonlinear terminations is considered. Figure 7.4 shows the p.u.l. resistance and inductance as function of frequency. The MRA macromodel (Padé order 8/8) is used to model each interconnect. The total size of the MNA equations including the Padé interconnect stamps is 4352 variables. Performing the passive reduction scheme, a macromodel containing 184 variables is obtained. Figure 7.5 show a comparison of Y-parameters of the linear subnetwork, which are obtained using SPICE analysis and the proposed macromodel. Figure 7.6 presents a comparison of time responses, at output nodes Vp2 and Vout. The input pulse used for this example has a rise/fall time of $0.1 \mathrm{~ns}$ and pulse width of $5 \mathrm{~ns}$. On a SunUltra- 5 computer it takes $1.7 \mathrm{~min}$ utes to obtain the transient response with the reduced model, while the conventional lumped system requires 80.5 minutes (Table 7.2)
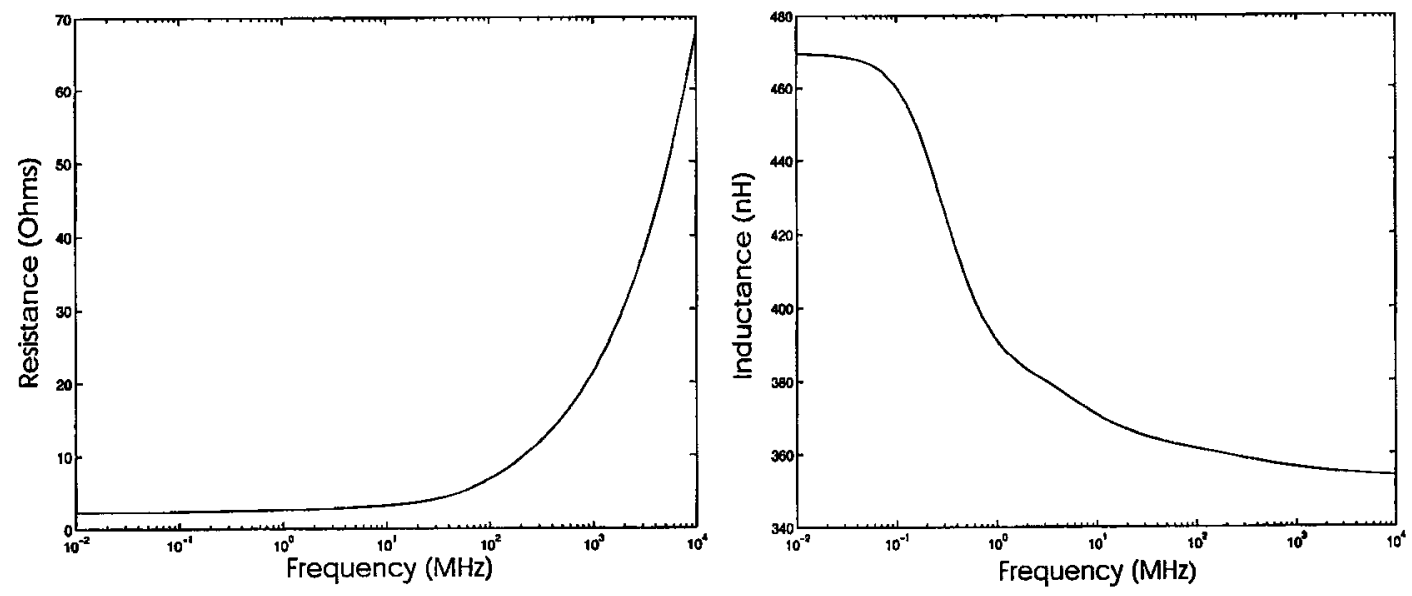

Figure 7.4 $\mathrm{R}$ and $\mathrm{L}$ versus frequency (Example 7.2)

\begin{tabular}{|c|c|c|}
\hline $\begin{array}{c}\text { Proposed Method } \\
\text { (minutes) }\end{array}$ & $\begin{array}{c}\text { Conventional lumped model } \\
\text { (minutes) }\end{array}$ & Speed-up \\
\hline 1.7 & 80.5 & 47 \\
\hline
\end{tabular}

Table 7.2 CPU comparison of transient response (Example 7.2) 


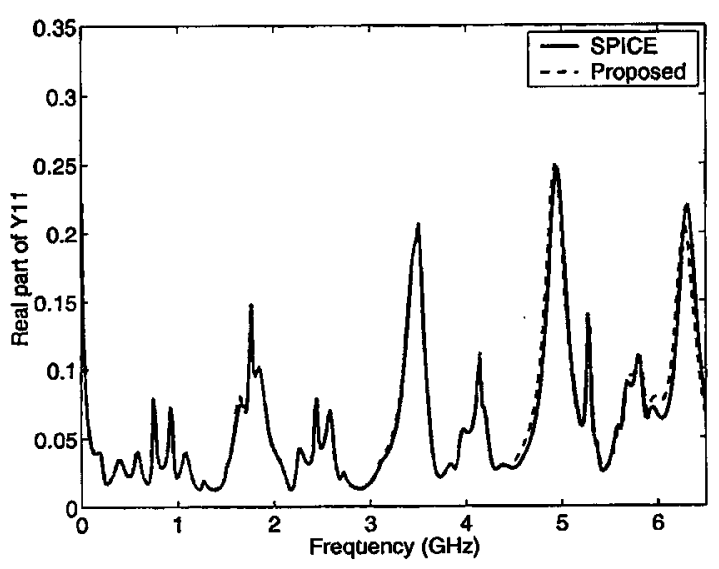

a) Real part of Y11

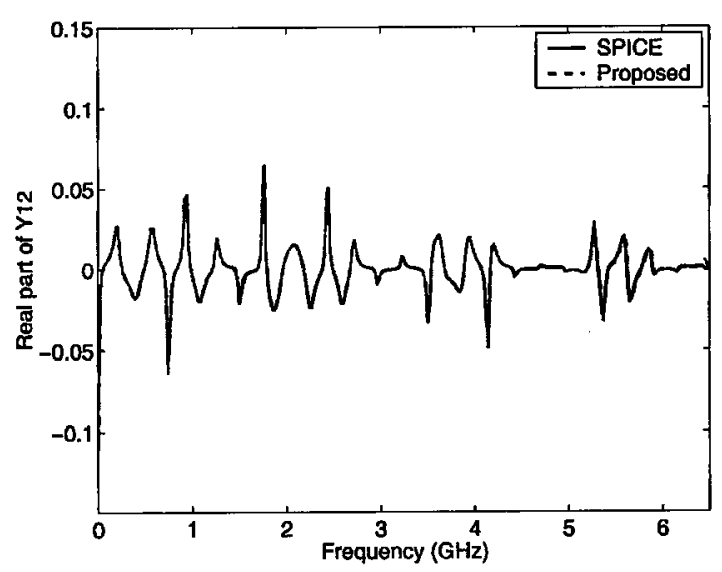

c) Real part of Y12

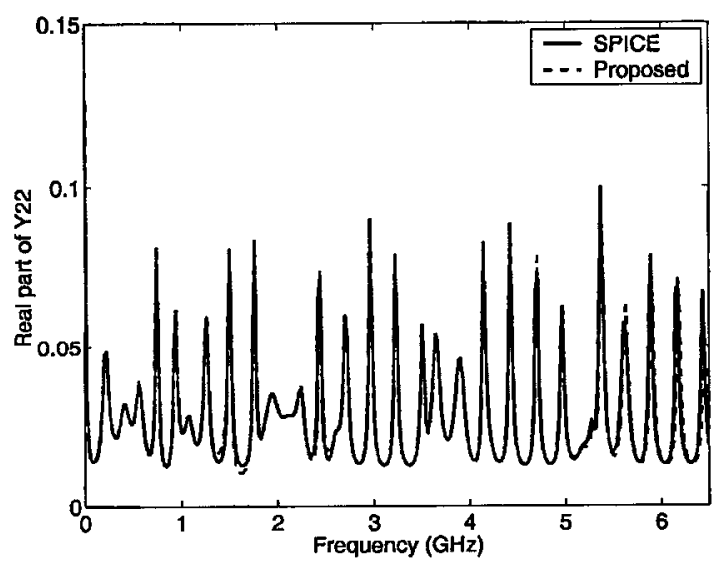

e) Real part of Y22

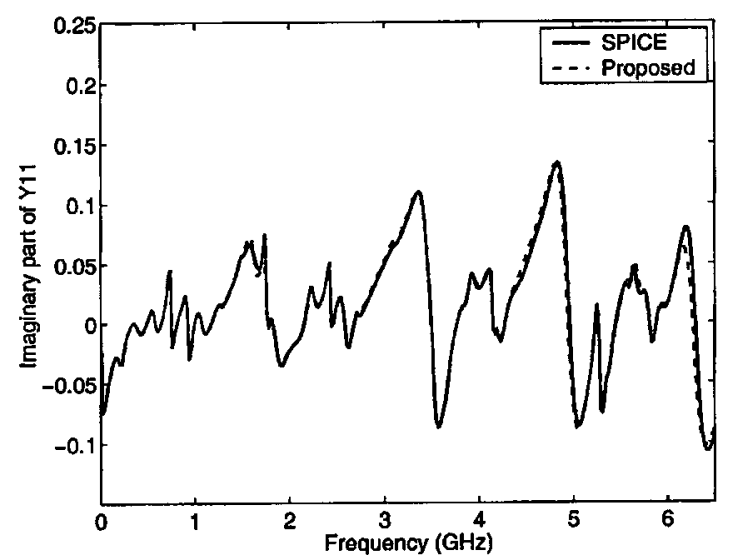

b) Imaginary part of Y11

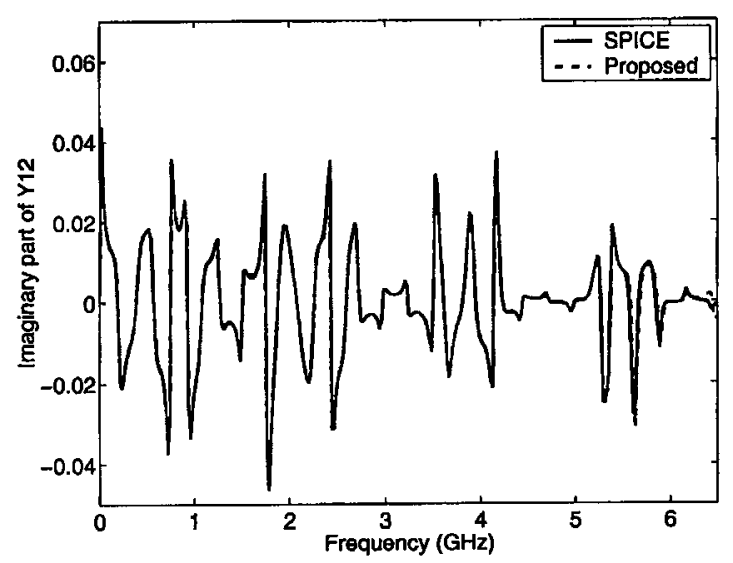

d) Imaginary part of Y12

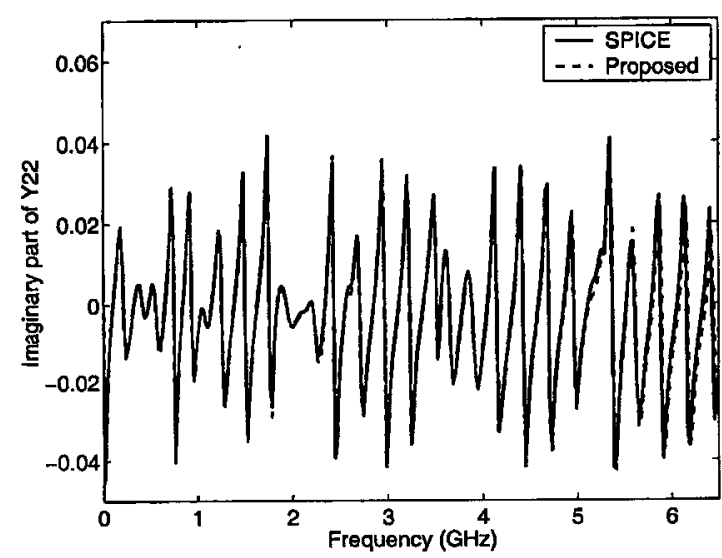

f) Imaginary part of $Y 22$

Figure 7.5 Y-parameters of linear subnetwork (Example 7.2) 


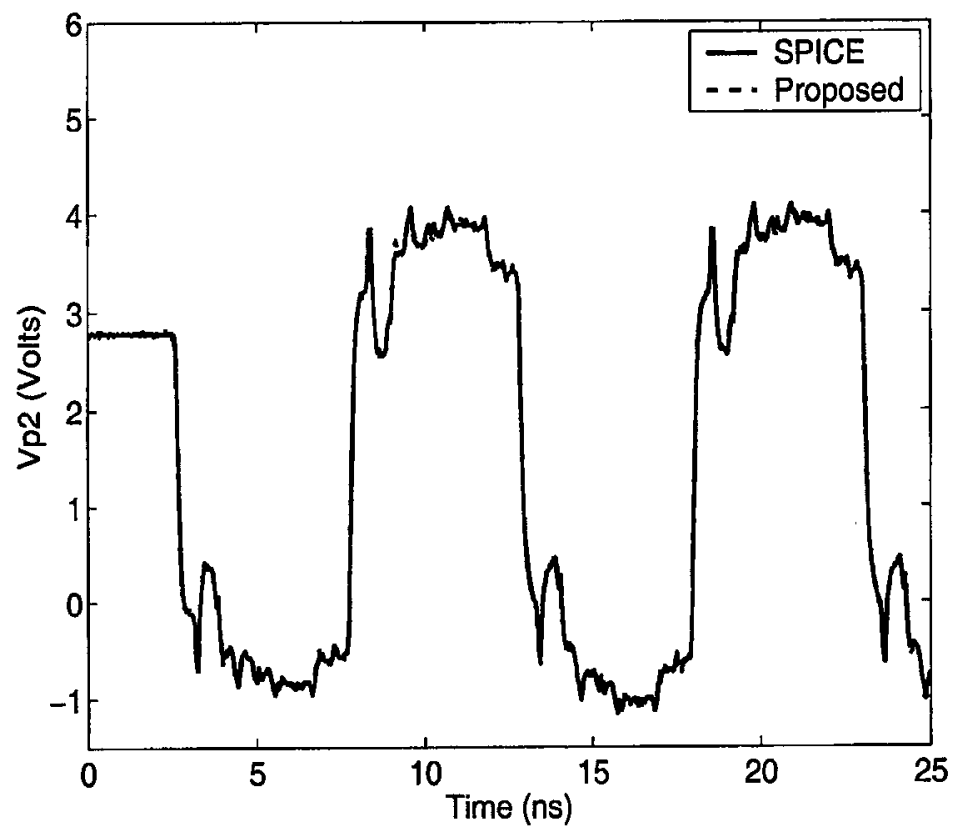

a) At port Vp2

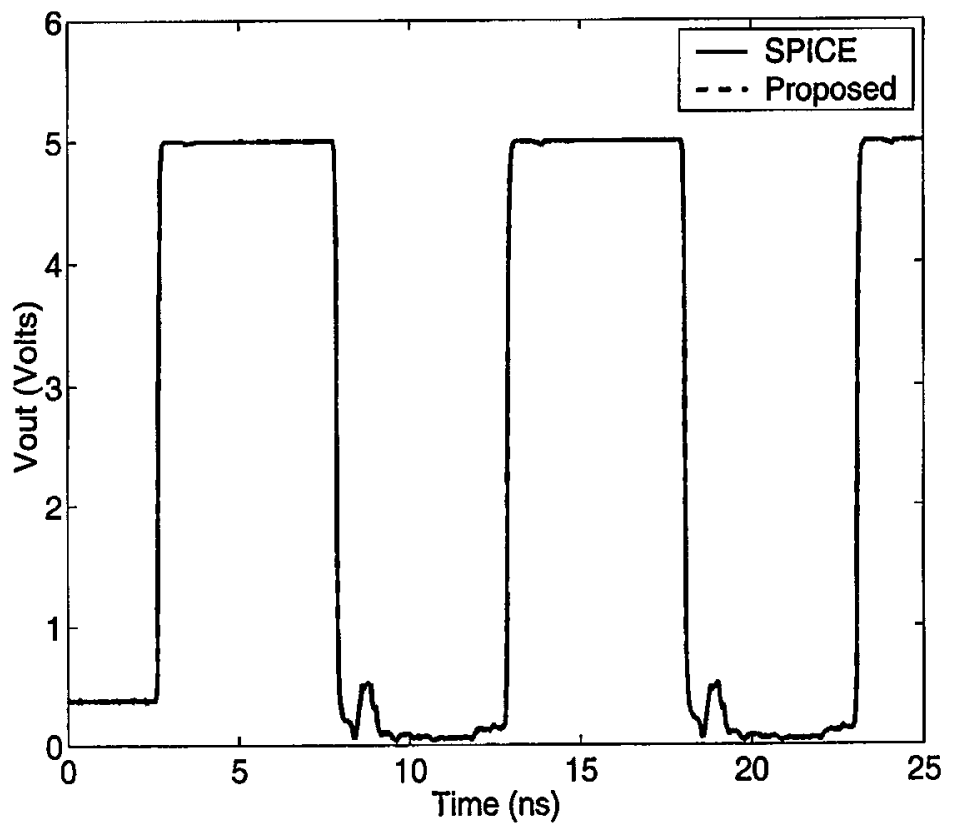

b) At node Vout

Figure 7.6 Transient response (Example 7.2) 


\section{Chapter 8}

\section{Sensitivity Analysis of Transmission Lines with Nonlinear Terminations}

\subsection{Introduction}

Efficient and accurate sensitivity information with respect to transmission line parameters is often required by optimizers to solve large nonlinear circuits with embedded distributed subnetworks [43]-[45]. In this chapter, an efficient approach to perform sensitivity analysis of nonlinear circuits with distributed transmission lines is presented. The proposed algorithm uses the matrix rational approximation (MRA) macromodel to describe transmission lines as well as to derive the network sensitivity with respect to any circuit parameter [59]-[61]. The method enables sensitivity analysis of transmission lines with respect to both electrical and physical parameters. A major advantage of the proposed method is that the derivatives of the circuit matrices are obtained analytically, in terms of predetermined coefficients and per-unit-length (p.u.l.) parameters, facilitating efficient formulation of the sensitivity network.

This chapter is organized as follows. The formulation of the network equations is presented in section 8.2. Section 8.3 outlines the steps involved for the sensitivity analysis of linear and nonlinear networks. From this discussion the proposed algorithm for sensitivity analysis of nonlinear networks with distributed transmission line components is presented. Numerical examples are provided in section 8.4. 


\subsection{Formulation of Network Equations}

As mentioned in the previous chapter, the MRA macromodel converts the distributed linear network of (7.1) to

$$
C_{\pi} \frac{d}{d t} \boldsymbol{x}_{\pi}(t)+\boldsymbol{G}_{\pi} \boldsymbol{x}_{\pi}(t)=\boldsymbol{b}_{\pi}(t)
$$

where the distributed transmission line is embedded in the modified nodal analysis (MNA) matrices of the overall network. For the case of a nonlinear distributed network, (8.1) becomes

$$
C_{\pi} \frac{d}{d t} x_{\pi}(t)+G_{\pi} x_{\pi}(t)+f_{\pi}\left(x_{\pi}(t)\right)=b_{\pi}(t)
$$

where $f_{\pi}\left(\boldsymbol{x}_{\pi}(t)\right)$ contains the nonlinear functions describing the nonlinear circuit elements. The matrices $G_{\pi}$ and $C_{\pi}$ can be written as

$$
G_{\pi}=G_{a}+G_{T} \quad C_{\pi}=C_{a}+C_{T}
$$

where $\boldsymbol{G}_{\boldsymbol{a}}$ and $\boldsymbol{C}_{\boldsymbol{a}}$ correspond to the MNA equations of the lumped components and $\boldsymbol{G}_{\boldsymbol{T}}$ and $C_{T}$ correspond to the stamp of the transmission line. For the circuit realization described in Chapter 4, the matrices $G_{T}$ and $C_{T}$ can be expressed as

$$
\boldsymbol{G}_{T}=\sum_{i}\left(\psi_{i}\right)^{\mathbf{T}} G_{i} \Psi_{i} \quad C_{T}=\sum_{i}\left(\psi_{i}\right)^{\mathbf{T}} C_{i} \psi_{i}
$$

where $\psi_{i}$ is a selector matrix that maps the block matrices $G_{i}$ and $C_{i}$ to the rest of the network equations and the superscript $T$ denotes the transpose of a matrix. The matrices $\boldsymbol{G}_{i}$ and $\boldsymbol{C}_{\boldsymbol{i}}$ are described in detail in chapters 4 and 5 for the various p.u.1. parameter models (i.e RC, lossless, lossy (RLGC) and frequency-dependent parameters). Substituting (8.4) into (8.3), gives

$$
G_{\pi}=G_{a}+\sum_{i}\left(\psi_{i}\right)^{\mathbf{T}} G_{i} \psi_{i} \quad C_{\pi}=C_{a}+\sum_{i}\left(\psi_{i}\right)^{\mathbf{T}} C_{i} \Psi_{i}
$$

In the next section, the sensitivity analysis of transmission lines networks is described using the MRA macromodel. 


\subsection{Sensitivity Analysis}

In this section, the sensitivity analysis is derived for both linear and nonlinear networks. From this discussion, the sensitivity network with respect to transmission line parameters is described for the MRA macromodel.

\subsubsection{Sensitivity Analysis of Linear Circuits}

If distributed networks are not in the presence of nonlinear elements, then (8.1) can be expressed in the frequency-domain as

$$
\left(s C_{\pi}+G_{\pi}\right) X_{\pi}=B_{\pi}
$$

where $\boldsymbol{X}_{\pi}$ is the Laplace transform of $\boldsymbol{x}_{\pi}(t)$ and $\boldsymbol{B}_{\pi}$ is the Laplace transform of $\boldsymbol{b}_{\pi}(t)$. An efficient method to obtain the sensitivities of the network is the adjoint technique [73]. Let $\Phi$ be the output variable of interest, defined as

$$
\Phi=z^{T} X_{\pi}
$$

where $z$ is a selector vector with unity entry corresponding to the output variable. Differentiating (8.6) and (8.7) with respect to a circuit parameter $\lambda$ and combining the two relations yields

$$
\frac{\partial \Phi}{\partial \lambda}=-\boldsymbol{X}_{a}^{T}\left(s \frac{\partial C_{\pi}}{\partial \lambda}+\frac{\partial G_{\pi}}{\partial \lambda}\right) \boldsymbol{X}_{\pi}
$$

where $\boldsymbol{X}_{a}$ is the solution of the adjoint network and is defined as

$$
\left(s C_{\pi}+G_{\pi}\right)^{T} X_{a}=z
$$

From (8.8), the sensitivity of an output variable with respect to a circuit parameter depends on $\boldsymbol{X}_{\pi}, \boldsymbol{X}_{a}, \partial \boldsymbol{C}_{\pi} / \partial \lambda$ and $\partial \boldsymbol{G}_{\pi} / \partial \lambda$. The values of $\boldsymbol{X}_{\pi}$ and $\boldsymbol{X}_{a}$ are obtained from the solution of the network and adjoint equations given by (8.6) and (8.9), respectively. For the case where $\lambda$ is a transmission line parameter, the matrices $\partial C_{\pi} / \partial \lambda$ and $\partial G_{\pi} / \partial \lambda$ are derived from the equations of the transmission line macromodel. How to derive these matrices from the MRA macromodel is described in section 8.3.4. 


\subsubsection{Sensitivity Analysis with Nonlinear Terminations}

The sensitivity of the nonlinear network with respect to a circuit parameter $\lambda$ is obtained by differentiating (8.2) with $\lambda$ as

$$
C_{\pi} \frac{d}{d t} z(t)+\left(G_{\pi}+\frac{\partial f_{\pi}\left(x_{\pi}(t)\right)}{\partial x_{\pi}(t)}\right) z(t)+E(t)=0
$$

where

$$
\begin{gathered}
E(t)=\frac{\partial C_{\pi}}{\partial \lambda} \frac{d}{d t} x_{\pi}(t)+\frac{\partial G_{\pi}}{\partial \lambda} x_{\pi}(t)+\frac{\partial f_{\pi}\left(x_{\pi}(t)\right)}{\partial \lambda} \\
z=\frac{\partial x_{\pi}(t)}{\partial \lambda}
\end{gathered}
$$

Solution of (8.2) and (8.10) can be obtained by converting them to difference equations using integration formulae such as backward Euler or trapezoidal rule [73]. For example, if trapezoidal rule is used (8.2) and (8.10) become

$$
\begin{aligned}
& A X_{n+1}+\frac{f\left(X_{n+1}\right)}{2}=B X_{n}-\frac{f\left(X_{n}\right)}{2}+\frac{\left(b_{n+1}+b_{n}\right)}{2} \\
& \left(A+\frac{\partial f\left(X_{n+1}\right)}{2 \partial X_{n+1}}\right) \frac{\partial X_{n+1}}{\partial \lambda}=-\frac{\partial A}{\partial \lambda} X_{n+1}+B \frac{\partial X_{n}}{\partial \lambda} \\
& +\frac{\partial B}{\partial \lambda} X_{n}-\frac{\partial f\left(X_{n}\right) \partial X_{n}}{2 \partial X_{n}} \frac{1}{\partial \lambda}\left(\frac{\partial f\left(X_{n+1}\right)}{\partial \lambda}+\frac{\partial f\left(X_{n}\right)}{\partial \lambda}\right)
\end{aligned}
$$

where

$$
A=\frac{C_{\pi}}{\Delta t}+\frac{G_{\pi}}{2} \quad B=\frac{C_{\pi}}{\Delta t}-\frac{G_{\pi}}{2}
$$

and $\boldsymbol{X}_{n}=\boldsymbol{X}_{\pi}\left(t_{n}\right)$. The variable $\Delta t$ is the time step between the time intervals $t_{n}$ to $t_{n+1}$.

Equations (8.12) and (8.13) represent the solution of the original and sensitivity networks as described by (8.2) and (8.10). The coefficients on the right side of (8.13) are all known from the solution of (8.12). The variables $\partial f\left(X_{n+1}\right) / \partial X_{n+1}$ and $\partial f\left(X_{n}\right) / \partial X_{n}$ are the Jacobean matrices which can be obtained by solving (8.12). The matrices $\partial A / \partial \lambda$ 
and $\partial B / \partial \lambda$ are derived from $\partial C_{\pi} / \partial \lambda$ and $\partial G_{\pi} / \partial \lambda$. For the case when $\lambda$ is a transmission line parameter, $\partial C_{\pi} / \partial \lambda$ and $\partial G_{\pi} / \partial \lambda$ are derived from the equations of the transmission line macromodel. How to obtain these matrices from the MRA macromodel is described in section 8.3.4.

\subsubsection{Sensitivity with Respect to Physical Transmission Line Parameters}

When studying the sensitivity of distributed networks, the design parameters of transmission lines are usually required with respect to physical parameters (such as width and spacing of conductors). The sensitivities of electrical parameters are often intermediate steps to the calculation of sensitivities of physical parameters. In the case where $\lambda$ represents a physical parameter of a transmission line network, the sensitivity of the output nodes can be obtained as follows:

$$
\frac{\partial x_{\pi}}{\partial \lambda}=\sum_{i=1}^{m} \sum_{j=1}^{m}\left(\frac{\partial x}{\partial R_{i, j}} \frac{\partial R_{i, j}}{\partial \lambda}+\frac{\partial x}{\partial L_{i, j}} \frac{\partial L_{i, j}}{\partial \lambda}+\frac{\partial x}{\partial G_{i, j}} \frac{\partial G_{i, j}}{\partial \lambda}+\frac{\partial x}{\partial C_{i, j}} \frac{\partial C_{i, j}}{\partial \lambda}\right)
$$

where $R_{i, j}, L_{i, j}, G_{i, j}$ and $C_{i, j}$ are the p.u.l. parameters. The subscripts $i$ and $j$ are matrix indices and $m+1$ is the number of conductors ( $m$ transmission lines plus one reference line).

\subsubsection{Calculating the derivatives of the MNA matrices}

To calculate the sensitivity of the network with respect to a transmission line parameter, the matrices $\partial C_{\pi} / \partial \lambda$ and $\partial G_{\pi} / \partial \lambda$ are required. The calculation of $\partial C_{\pi} / \partial \lambda$ and $\partial G_{\pi} / \partial \lambda$ depends on the transmission line model being used. For the case of the MRA macromodel, the matrices are obtained as follows. Let $\lambda$ be a transmission line parameter. Differentiating the matrices of (8.5) with respect to $\lambda$ yields

$$
{\frac{\partial G_{\pi}}{\partial \lambda}}^{n}=\sum_{i}\left(\psi_{i}\right)^{T} \frac{\partial G_{i}}{\partial \lambda_{k}} \psi_{i}^{k} \quad \frac{\partial C_{\pi}}{\partial \lambda}=\sum_{i}\left(\psi_{i}\right)^{T} \frac{\partial C_{i}}{\partial \lambda_{k}} \psi_{i}
$$

The matrices $\partial C_{i} / \partial \lambda$ and $\partial G_{i} / \partial \lambda$ are computed analytically in terms of p.u.1. param- 
eters and predetermined coefficients given by the MRA. This makes the formulation of sensitivity networks extremely simple when compared to other transmission line macromodels. As an example, Table 8.1 shows how these matrices are obtained for a two-conductor lossy transmission line for the subsection described by (4.27). For the MRA macromodel described in Chapter 6, the sensitivity network is also computed in a similar manner.

\begin{tabular}{|c|c|}
\hline$\partial G_{i} / \partial \lambda$ & $\partial C_{i} / \partial \lambda$ \\
\hline$\frac{\partial G_{0}}{\partial d}=\left[\begin{array}{cccc}\frac{G}{2 \alpha_{0}} & 0 & \frac{G}{2 \alpha_{0}} & 0 \\
0 & \frac{-\alpha_{0}}{2 d^{2} R} & \frac{\alpha_{0}}{2 d^{2} R} & 0 \\
\frac{G}{2 \alpha_{0}} & \frac{\alpha_{0}}{2 d^{2} R}\left(\frac{-\alpha_{0}}{2 d^{2} R}+\frac{G}{2 \alpha_{0}}\right) & 0 \\
0 & 0 & 0 & 0\end{array}\right]$ & $\frac{\partial C_{0}}{\partial d}=\left[\begin{array}{cccc}\frac{C}{2 \alpha_{0}} & 0 & \frac{C}{2 \alpha_{0}} & 0 \\
0 & 0 & 0 & 0 \\
\frac{C}{2 \alpha_{0}} & 0 & \frac{C}{2 \alpha_{0}} & 0 \\
0 & 0 & 0 & \frac{2 L}{\alpha_{0}}\end{array}\right]$ \\
\hline$\frac{\partial G_{0}}{\partial R}=\left[\begin{array}{cccc}0 & 0 & 0 & 0 \\
0 & \frac{-\alpha_{0}}{2 d R^{2}} & \frac{\alpha_{0}}{2 d R^{2}} & 0 \\
0 & \frac{\alpha_{0}}{2 d R^{2}} & \frac{-\alpha_{0}}{2 d R^{2}} & 0 \\
0 & 0 & 0 & 0\end{array}\right]$ & $\frac{\partial C_{0}}{\partial R}=\left[\begin{array}{llll}0 & 0 & 0 & 0 \\
0 & 0 & 0 & 0 \\
0 & 0 & 0 & 0 \\
0 & 0 & 0 & 0\end{array}\right]$ \\
\hline$\frac{\partial G_{0}}{\partial G}=\left[\begin{array}{cccc}\frac{d}{2 \alpha_{0}} & 0 & \frac{d}{2 \alpha_{0}} & 0 \\
0 & 0 & 0 & 0 \\
\frac{d}{2 \alpha_{0}} & 0 & \frac{d}{2 \alpha_{0}} & 0 \\
0 & 0 & 0 & 0\end{array}\right]$ & $\frac{\partial C_{0}}{\partial G}=\left[\begin{array}{llll}0 & 0 & 0 & 0 \\
0 & 0 & 0 & 0 \\
0 & 0 & 0 & 0 \\
0 & 0 & 0 & 0\end{array}\right]$ \\
\hline$\frac{\partial G_{0}}{\partial C}=\left[\begin{array}{llll}0 & 0 & 0 & 0 \\
0 & 0 & 0 & 0 \\
0 & 0 & 0 & 0 \\
0 & 0 & 0 & 0\end{array}\right]$ & $\frac{\partial C_{0}}{\partial C}=\left[\begin{array}{cccc}\frac{d}{2 \alpha_{0}} & 0 & \frac{d}{2 \alpha_{0}} & 0 \\
0 & 0 & 0 & 0 \\
\frac{d}{2 \alpha_{0}} & 0 & \frac{d}{2 \alpha_{0}} & 0 \\
0 & 0 & 0 & 0\end{array}\right]$ \\
\hline$\frac{\partial G_{0}}{\partial L}=\left[\begin{array}{llll}0 & 0 & 0 & 0 \\
0 & 0 & 0 & 0 \\
0 & 0 & 0 & 0 \\
0 & 0 & 0 & 0\end{array}\right]$ & $\frac{\partial C_{0}}{\partial L}=\left[\begin{array}{cccc}0 & 0 & 0 & 0 \\
0 & 0 & 0 & 0 \\
0 & 0 & 0 & 0 \\
0 & 0 & 0 & \frac{2 d}{\alpha_{0}}\end{array}\right]$ \\
\hline
\end{tabular}

Table 8.1 Example showing calculations of sensitivity network 


\subsection{Examples}

Two examples are presented in this section to demonstrate the validity and efficiency of the proposed sensitivity analysis approach for nonlinear distributed networks.

\subsubsection{Example 8.1}

A coupled transmission line system with nonlinear termination is shown in Figure 8.1. The length of the line is $4 \mathrm{~cm}$ and the p.u.l. parameters are

$$
\boldsymbol{R}=\left[\begin{array}{cc}
0.14 & 0 \\
0 & 0.14
\end{array}\right] \Omega / \mathrm{cm} \quad \boldsymbol{L}=\left[\begin{array}{cc}
6.3 & 2.9 \\
2.9 & 6.3
\end{array}\right] \mathrm{nH} / \mathrm{cm} \quad \boldsymbol{C}=\left[\begin{array}{cc}
1.1 & -0.45 \\
-0.45 & 1.1
\end{array}\right] \mathrm{pF} / \mathrm{cm}
$$

In this example, the off diagonal p.u.l. resistances are zero since the return line is assumed to be lossless. Figure 8.2 shows transient responses of the far-end voltages corresponding to a 5 Volt input pulse with rise/fall times $0.1 \mathrm{~ns}$ and a pulse width of $0.8 \mathrm{~ns}$. The sensitivities with respect to $C_{11}$ for the active, victim and Vout nodes are shown in Figure 8.3. The results of the proposed method are compared with the perturbation of the conventional lumped segment model [2] (referred to as SPICE Perturbation). Both the proposed method and the perturbation results are in good agreement.

It is to be noted that using the proposed method provides the following advantages:

1. Using the MRA macromodel provides significant CPU advantages compared to lumped segmentation model. For this example the total size of the MNA matrices was $94 \times 94$ for the proposed method while the size of the lumped model was $408 \times 408$.

2. Perturbation based techniques can lead to inaccurate results (depending on the magnitude of the perturbation).

3. In addition, the nonlinear differential equations representing the perturbed network must be solved separately for every parameter of interest. However, in the proposed approach, the sensitivity information with respect to all the parameters can be essentially obtained from the solution of the original network. 
Table 8.2 shows a comparison of savings in the main computational cost (in terms of total number of LU decompositions) using the proposed approach versus the perturbation approach, for the above example.

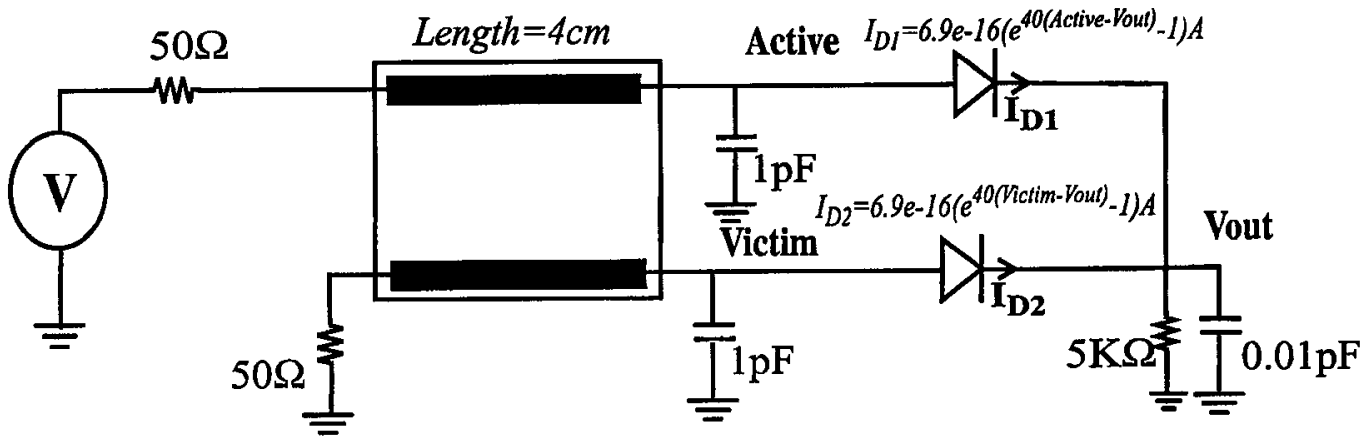

Figure 8.1 Transmission line system with nonlinear termination (Example 8.1)

\begin{tabular}{c|c|c}
\hline $\begin{array}{c}\text { \# of } \\
\text { parameters }\end{array}$ & $\begin{array}{c}\text { Perturbation (\# LU } \\
\text { decompositions) }\end{array}$ & $\begin{array}{c}\text { Proposed (\# LU } \\
\text { decompositions) }\end{array}$ \\
\hline 10 & 17501 & 1601 \\
\hline
\end{tabular}

Table 8.2 Computational Complexity: Proposed vs Perturbation (Example 8.1) 


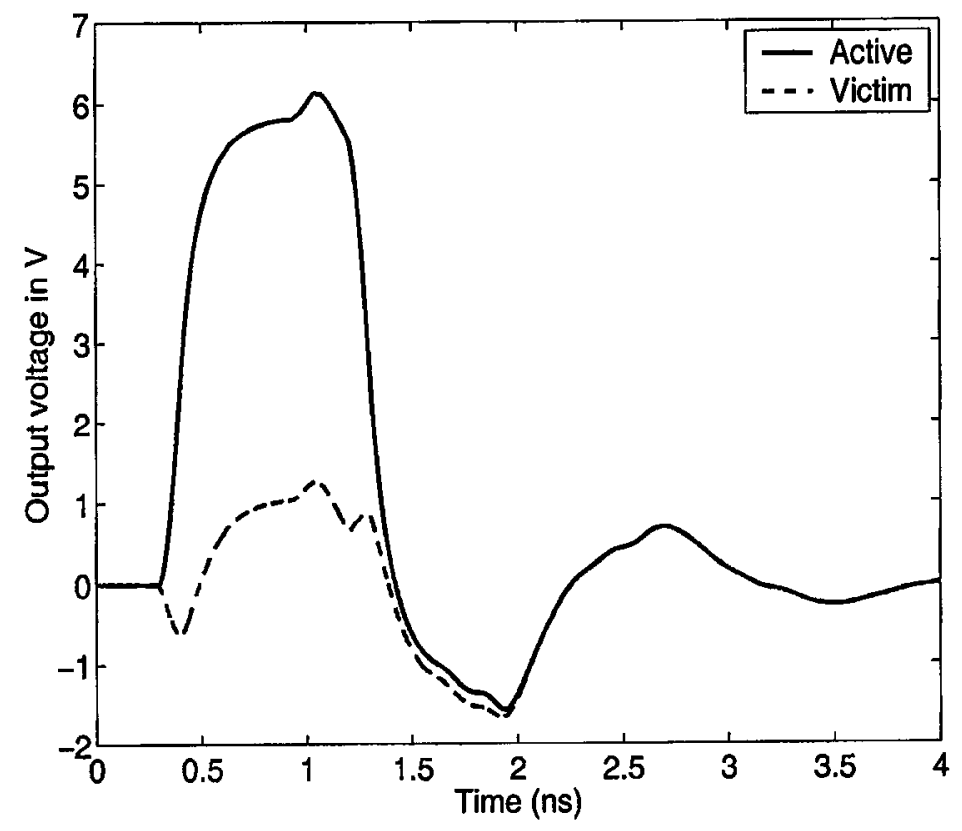

a) Response of active and victim line

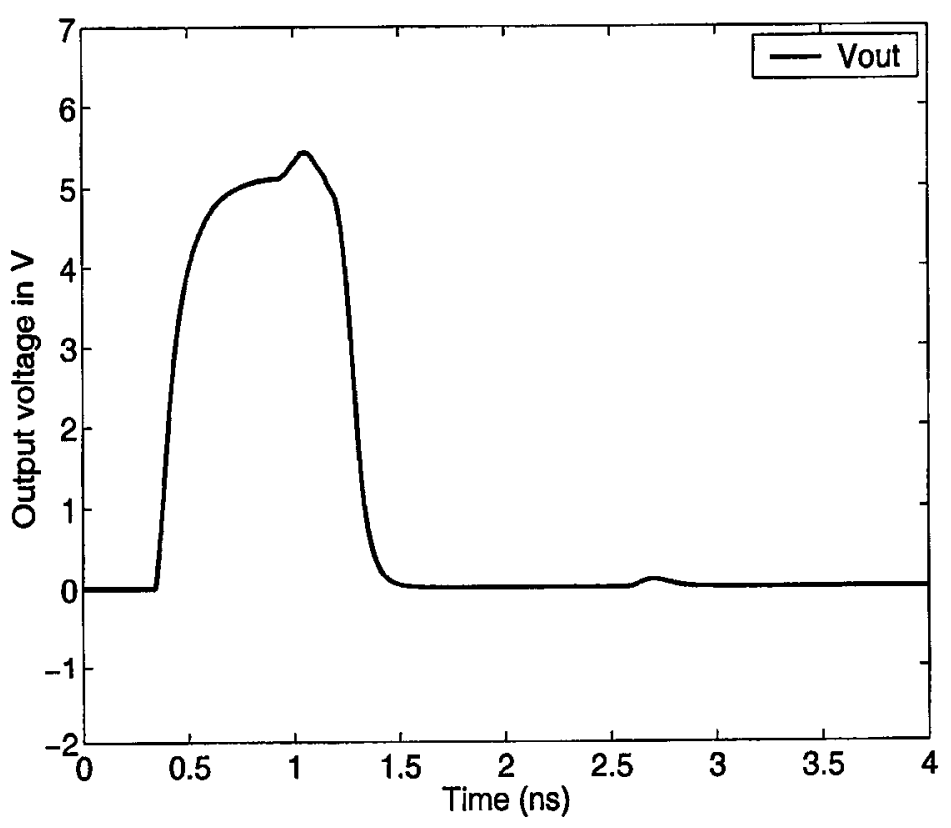

b) Response of Vout

Figure 8.2 Transient response of nonlinear circuit (Example 8.1) 


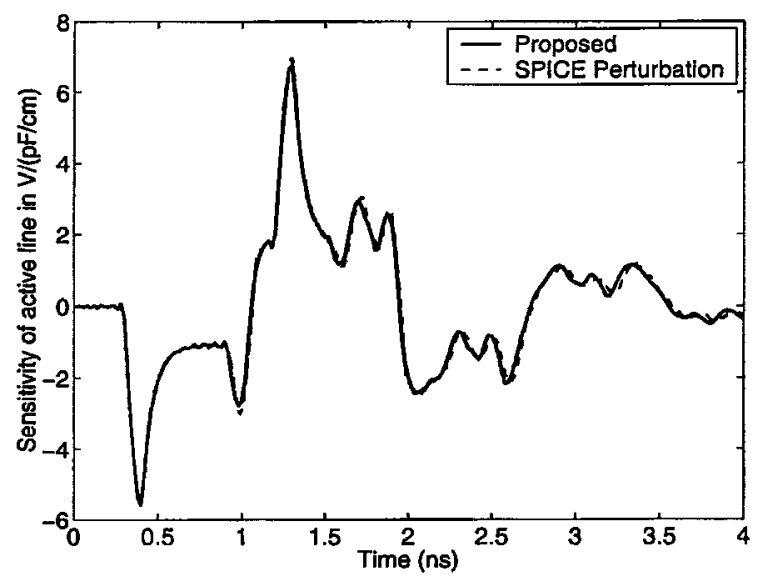

a) At active line

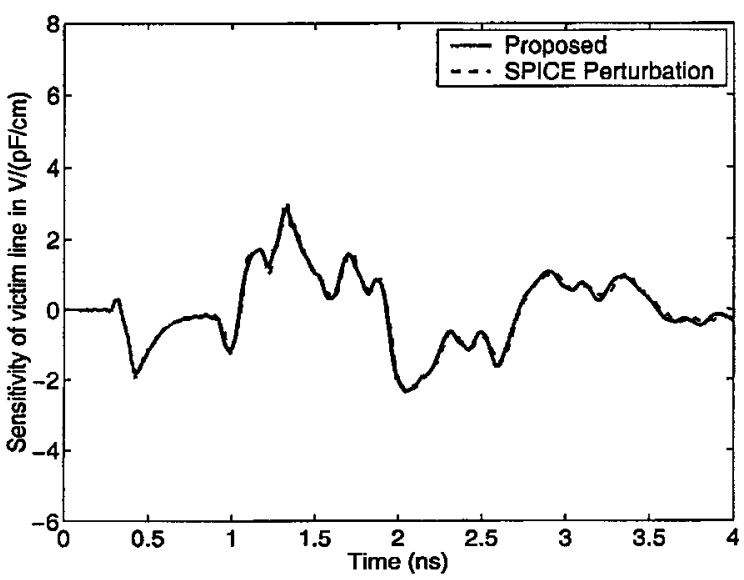

b) At victim line

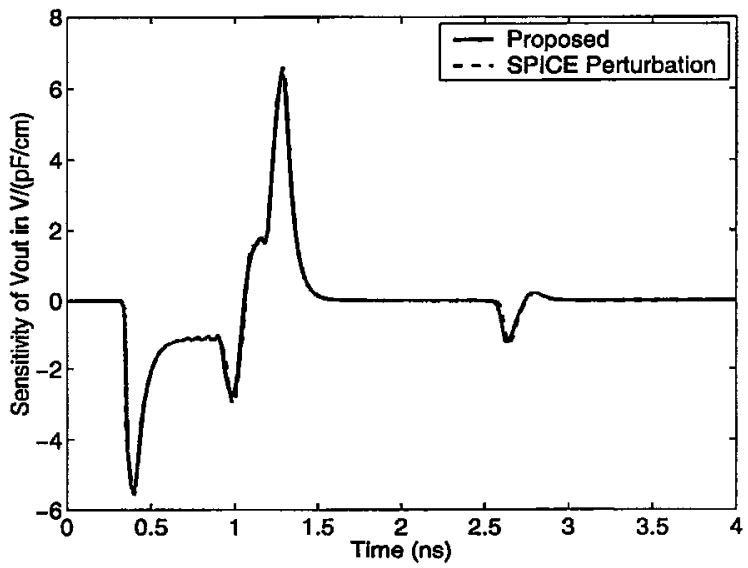

c) At Vout

Figure 8.3 Sensitivity with respect to $C_{11}$ 


\subsubsection{Example 8.2}

In this example, interconnect transmission line parameters are optimized to obtain desired circuit performance. A three-transmission line system with nonlinear terminations is shown in Figure 8.4. Figure 8.5 shows the interconnect design variables that are considered. The relative dielectric constant of the circuit board is 4.5. The p.u.l. parameters are computed from the physical description of the transmission line by using an electromagnetic modeling tool [85]. The results obtained are used to formulate a look-up table. Intermediate data points are interpolated using Stirling's formula [98]. This results in relatively more accurate p.u.l. parameters during the optimization process. A 5V step response with time-rise of lns is the input signal of the circuit. The responses of interest are nodes labeled $V_{1}, V_{2}, V_{3}$ and $V_{4}$. The responses before optimization are plotted in Figure 8.6. The delays of $V_{1}$ and $V_{3}$ are to be reduced to $5.5 \mathrm{~ns}$ and 5.0ns, respectively, based on a threshold voltage of $3.0 \mathrm{~V}$. In addition, it is desired to maintain the voltages of $V_{1}$ and $V_{3}$ greater then $4 \mathrm{~V}$ after $6.5 \mathrm{~ns}$ and $6.0 \mathrm{~ns}$, respectively. A $0.4 \mathrm{~V}$ upper specification is imposed on the magnitude of $V_{2}$ and $V_{4}$. The error functions for $V_{1}$ and $V_{3}$ become

$$
\begin{gathered}
e_{1}=\left[V_{1}(\phi, 5.5 n s)-3 V\right] \\
e_{2}=-\left[V_{1}(\phi, 5.5 n s)-3 V\right] \\
e_{3}=\left[V_{3}(\phi, 5.0 n s)-3 V\right] \\
e_{4}=-\left[V_{3}(\phi, 5.0 n s)-3 V\right] \\
e_{i+4}=-\left[V_{1}\left(\phi, t_{i}\right)-4 V\right] \\
e_{j+20}=-\left[V_{3}\left(\phi, t_{j}\right)-4 V\right]
\end{gathered}
$$

where $i=[1,2, \ldots, 16], j=[1,2, \ldots, 16], t_{i}$ and $t_{j}$ are equidistant time points between the interval $[6.5 n s, 40.0 n s]$ and $[6.0 n s, 40.0 n s]$, respectively. A weight factor of 10 is used to make the error functions of $V_{2}$ and $V_{4}$ comparable in scale with those of $V_{1}$ and $V_{3}$. The error functions $e_{37}$ to $e_{100}$ for $V_{2}$ and $V_{4}$ become 


$$
\begin{aligned}
e_{k+36} & =10\left[V_{2}\left(\phi, t_{k}\right)-0.4 V\right] \\
e_{k+52} & =-10\left[V_{2}\left(\phi, t_{k}\right)+0.4 V\right] \\
e_{k+68} & =10\left[V_{4}\left(\phi, t_{k}\right)-0.4 V\right] \\
e_{k+84} & =-10\left[V_{4}\left(\phi, t_{k}\right)+0.4 V\right]
\end{aligned}
$$

where $k=[1,2, \ldots, 16]$ and $t_{k}$ are equidistant time points between the interval $[0.0 n s, 40.0 n s]$. As seen from Figure 8.6, all specifications are violated before optimization.

The design variables $\phi$ include line width $w$, spacing between conductors $s$, circuit board thickness $h$, and lengths of multiconductor lines $d_{1}, d_{2}$ and $d_{3}$. Parameters $w, s$, and $h$ are the same for all three transmission lines. Design constraints of the circuit require that the total length of the three-transmission lines be $1.35 \mathrm{~m}$ and the total width of the two conductors plus the spacing between them be fixed $2.5 \mathrm{~mm}$. In addition, the width of the line has to be equal or greater then $0.1 \mathrm{~mm}$ and allowable range of the circuit board thickness is between $0.5 \mathrm{~mm}$ to $2.5 \mathrm{~mm}$. The constraints on the design variables are

$$
\begin{gathered}
g_{1}(\phi)=2 w+s-2.5 \mathrm{~mm} \\
g_{2}(\phi)=d_{1}+d_{2}+d_{3}-1.35 \mathrm{~m} \\
w \geq 0.1 \mathrm{~mm} \\
0.5 \mathrm{~mm} \leq h \leq 2.5 \mathrm{~mm}
\end{gathered}
$$

The initial values of the variables are $w=0.5 \mathrm{~mm}, s=1.5 \mathrm{~mm}, h=2.0 \mathrm{~mm}, d_{1}=0.45 \mathrm{~m}$, $d_{2}=0.45 \mathrm{~m}$ and $d_{3}=0.45 \mathrm{~m}$. The design variables after optimization are $w=0.149 \mathrm{~mm}$, $s=2.202 \mathrm{~mm}, h=0.998 \mathrm{~mm}, d I=0.132 \mathrm{~m}, d 2=0.651 \mathrm{~m}$ and $d 3=0.567 \mathrm{~m}$. The optimized circuit responses are juxtaposed against those before optimization in Figure 8.6. The new design variables meet all the circuit specifications without violating any design constraint. 


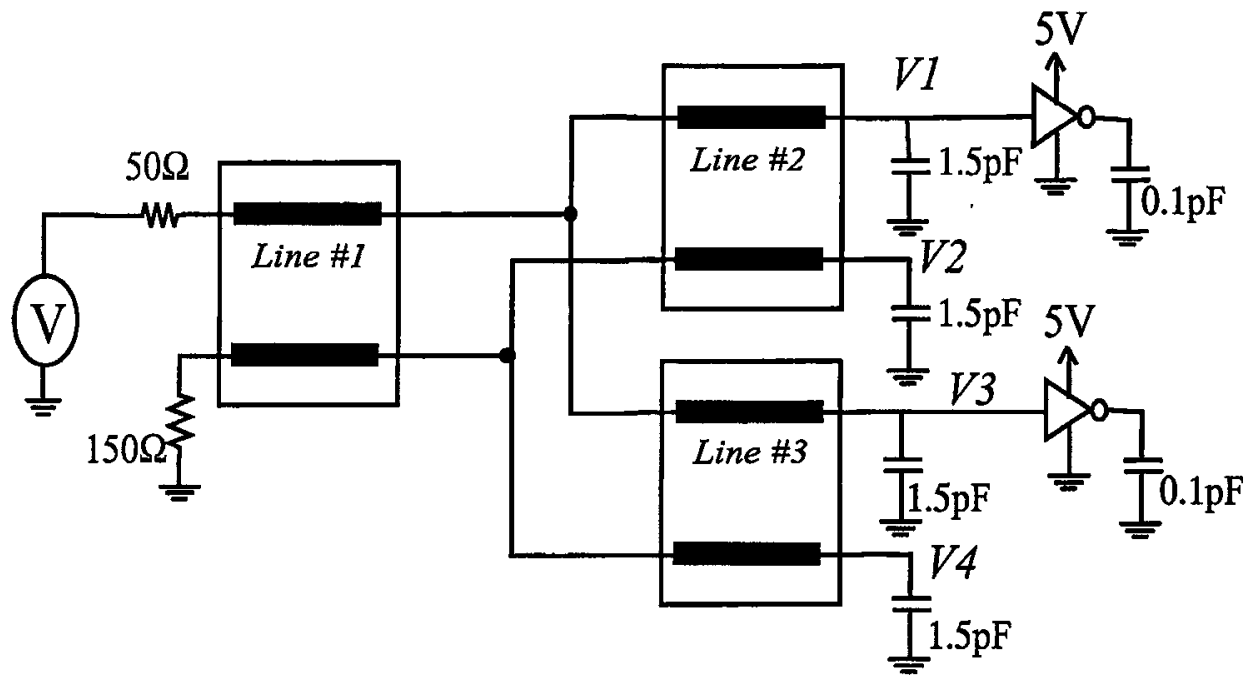

Figure 8.4 Three coupled transmission lines with nonlinear terminations

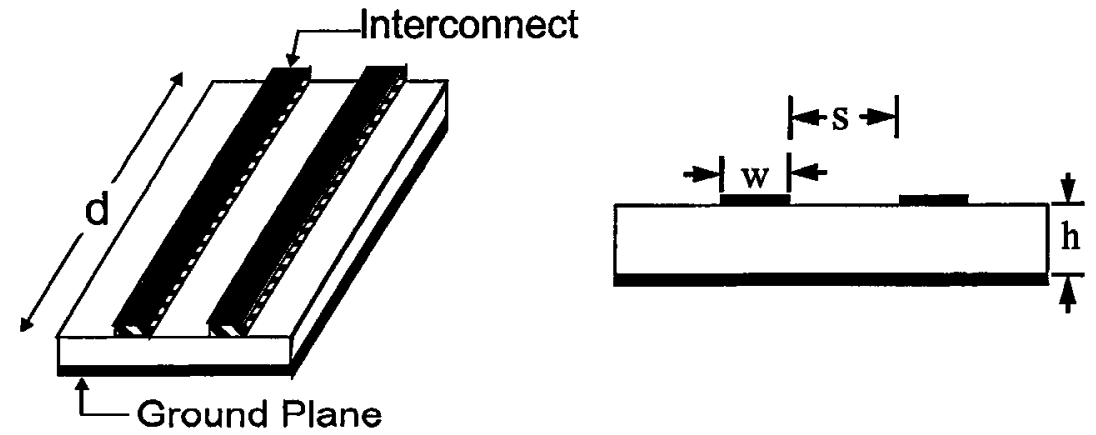

Figure 8.5 Physical/geometrical parameters of microstrip line 


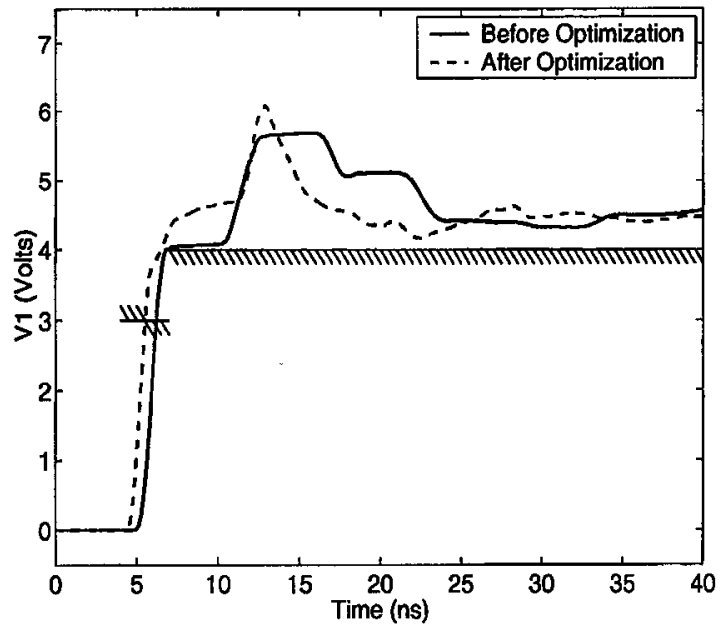

a) Sign response at $\mathrm{V} 1$

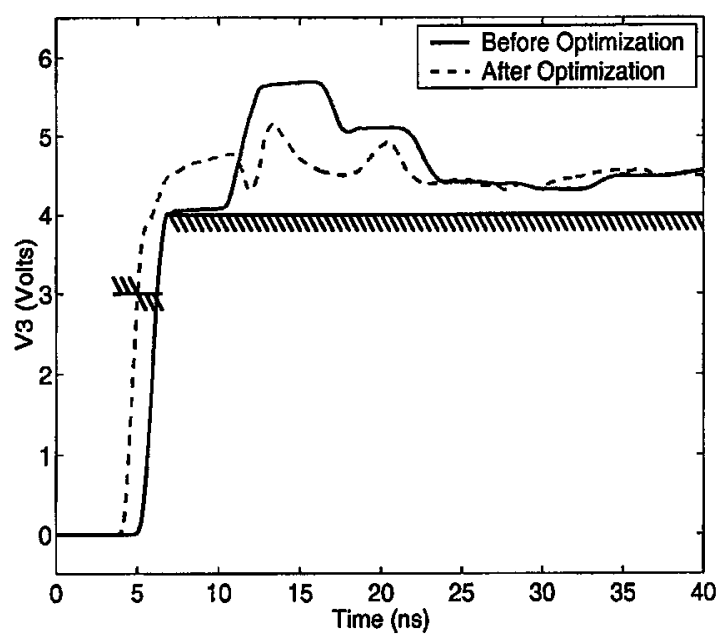

c) Sign response at V3

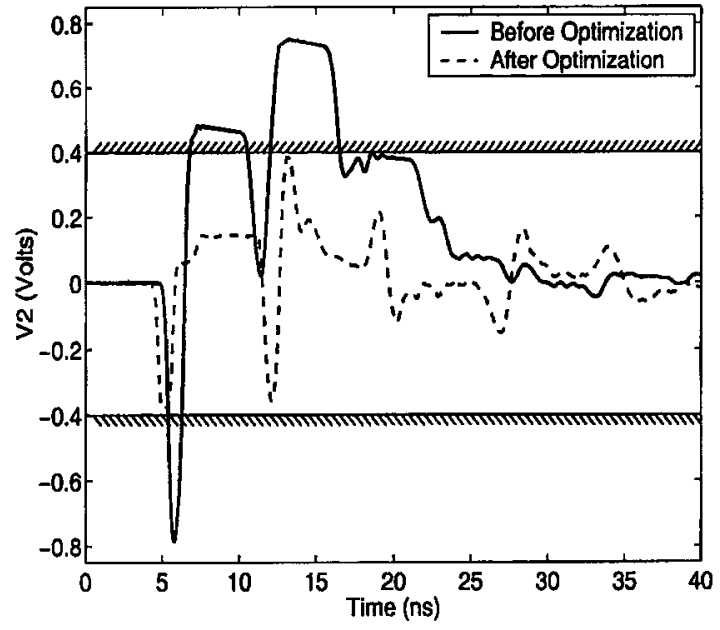

b) Sign response at V2

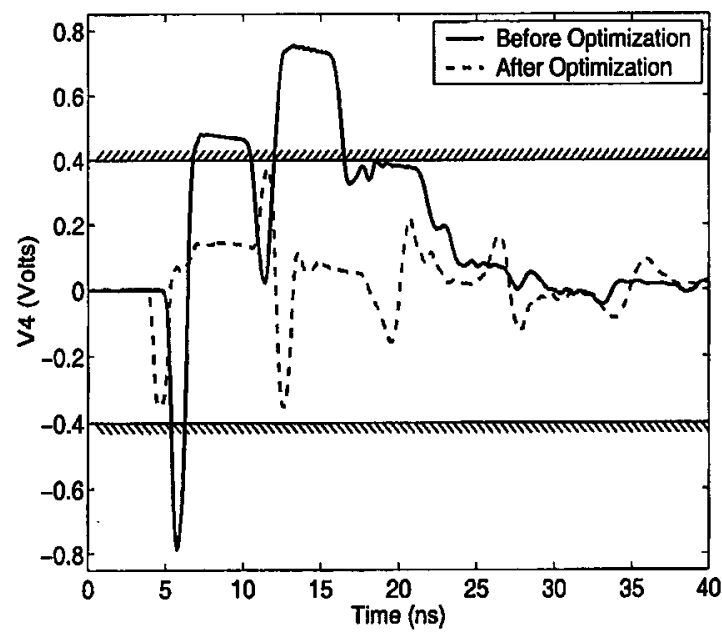

d) Sign response at V4

Figure 8.6 Signal response before and after optimization (example 8.2) 


\section{Chapter 9}

\section{Summary and Future Work}

\subsection{Summary}

The objective of this thesis is to develop efficient algorithms to model high-speed interconnects in a circuit simulation environment. The proposed algorithm is based on a matrix rational approximation (MRA) used to describe the solution the transmission line equations [46]-[69]. In this section, the contributions of this thesis are summarized.

One of the key issues in modeling transmission lines is the preservation of passivity. Since transmission lines are passive elements, passivity is an important property to satisfy because stable but not passive macromodels can produce unstable networks when connected to other passive loads. This thesis describes a general class of passive macromodeling algorithm for multi-port transmission-lines (MTLs) [46]-[50]. A new theorem is presented that specifies sufficient conditions for the MRA macromodel to generate passive macromodels. In addition, classes of rational approximations are provided that satisfy the conditions of the passivity theorem. The time-domain formulation of the MRA macromodel is provided for the RC, lossless and lossy transmission line models. A key feature of the MRA algorithm is that the macromodel can be formulated analytically in terms of predetermined coefficients and the per-unit-length (p.u.l.) parameters. This simplifies the formulation of the MRA algorithm compared to other transmission line macromodels that 
require numerical and/or optimization to compute the modified nodal analysis (MNA) equations describing the transmission line.

A technique to include transmission lines with frequency-dependent parameters is described [52]. To preserve passivity, the method uses positive real rational functions to model the frequency-dependent parameters. A curve fitting technique to match the frequency-dependent parameters to positive real rational functions is described. Also the time-domain formulation of the MRA macromodel is provided for transmission lines with frequency-dependent parameters. It should be noted that with the knowledge of the rational functions of the p.u.1. parameters, the MRA algorithm is formulated analytically in terms of predetermined coefficients and the p.u.1. parameter functions.

Application of the MRA macromodel to include incident field coupling of lossy MTLs is presented. The MRA algorithm is used to model both the transmission line and incident fields. The macromodel takes the form of ordinary differential equation (ODEs) and can easily be included in a circuit simulation environment [64]-[65].

A technique to minimize high-frequency errors in transient responses of rational macromodels is described [62]-[63]. The method provides a mechanism to control the asymptotic behaviour of the high-frequency impulse response while matching the response to the maximum frequency range of interest. The proposed technique is applied to the MRA algorithm, while preserving the passivity of the macromodel. In addition, the formulation of the macromodel is analytic in terms of predetermined coefficients and the p.u.l. parameters. The resulting macromodel leads to significant reduction in high-frequency transient errors for long delay and coupled transmission lines.

For large linear networks composed of many lumped and distributed elements, modelorder-reduction (MOR) techniques can be used to obtain efficient macromodels. The MRA algorithm is shown to be compatible with passive MOR techniques. A proof is provided showing that the contribution of the MRA stamp to the MNA formulation leads to a passive reduced-order macromodels [53]-[58].

An efficient approach for sensitivity analysis of MTLs in the presence of nonlinear ter- 
minations is described. The proposed method uses the MRA algorithm to obtain sensitivity information of nonlinear networks. An important feature of the proposed approach is that the derivatives of the MNA matrices with respect to the p.u.l. parameters are obtained analytically [59]-[61].

\subsection{Future Work}

This section provides some suggestions for further research based on work presented in this thesis.

1. A comparative study between the MRA algorithm and the method of characteristics suggest that each macromodel has its own strengths and weaknesses [66]-[68]. The MRA algorithm is able to enforce passivity and performs well for short lossy transmission lines such as on-chip interconnects as demonstrated in chapter 6, example 6.2. The method of characteristics uses delay extraction and is more efficient for long low-loss transmission lines such as long coaxial cables. However, the method of characteristics does not guarantee passivity for lossy transmission lines. The objective of the research would be to develop passive macromodels with delay extraction to obtain efficient and passive macromodels for long low-loss transmission lines.

2. The frequency-domain solution of non-uniform transmission lines cannot be expressed in terms of an exponential matrix as described by (3.2), since the p.u.l. parameters are function of position. This makes it difficult to use the MRA algorithm to model non-uniform transmission-lines. One-way of dealing with non-uniform transmission lines are to approximate them discretely as uniform transmission lines. However, this can lead to many segments, rendering the method inefficient. The development of a passive macromodel for non-uniform transmission lines that can be formulated analytically in terms of predetermined coefficients and the p.u.l. parameters would be an interesting project to pursue. 
3. A scheme to fit the data values of the p.u.1. parameters to positive real rational functions was described in chapter 5 . The proposed technique relied on a fixed circuit topology to obtain positive real rational functions. This approach works well, since the typical behaviour of the p.u.l. parameters are known. However, for arbitrary measured data the method can suffer from convergence problems since the appropriate circuit topology may not be known. The issue of passive macromodeling of arbitrary measured data is still a topic of research.

4. The MRA algorithm uses a scalar approximation of an exponential function to capture the frequency response of transmission lines. This technique can also be extended to derive other types of macromodels. For example, if the frequency response of devices can expressed in terms of some known function, then the concepts of the MRA algorithm can be applied to derive macromodels in terms of predetermined coefficients and the device parameters. 


\section{References}

[1] H. B. Bakoglu, Circuit, Interconnections and packaging for VLSI. Reading, MA: Addison-Wesley, 1990.

[2] C. R. Paul, Analysis of multiconductor transmission lines. New York, NY: John Wiely and Sons, 1994.

[3] R. Achar and M. Nakhla, "Simulation of high-speed Interconnects," Proceedings of the IEEE, vol. 49, pp. 693-728, May 2001.

[4] A. Deutsch, "Electrical characteristics of interconnections for high-performance systems," Proceedings of the IEEE, vol. 86, pp. 315-355, Feb. 1998.

[5] M. S. Nakhla, "Analysis of pulse propagation on high-speed VLSI chips," IEEE Journal of Solid-State Circuits, vol. 25, pp. 490-494, April 1990.

[6] L. T. Pillage and R. A. Rohrer, "Asymptotic waveform evaluation for timing analysis," IEEE Trans. Computer-Aided Design, vol. 9, pp.352-366, Apr. 1990.

[7] S. Y. Kim, N. Gopal and L. T. Pillage, "Time-domain macromodels for VLSI interconnect analysis," IEEE Trans. Computer-Aided Design, vol. 13, pp. 12571270, Oct. 1994.

[8] M. Celik and A. C. Cangellaris, "Efficient transient simulation of lossy packaging interconnects using moment-matching techniques," IEEE Trans. Comp. Packag. Manuf. Technol. B, vol. 19, pp. 64-73, Feb. 1996.

[9] E. Chiprout and M. S. Nakhla, "Analysis of interconnect networks using complex frequency hopping (CFH)," IEEE Trans. Computer-Aided Design, vol. 14, pp. 186-200, Feb. 1995.

[10] P. Feldmann and R. W. Freund, "Efficient linear circuit analysis by Padé approximation via Lanczos process," IEEE Trans. Computer-Aided Design, vol. 14, pp. 639-649, May 1995.

[11] M. Silveria, M. Kamon, I. Elfadel and J. White, "A coordinate-transformed Arnoldi algorithm for generating guaranteed stable reduced-order models of arbitrary RLC circuits", Proceedings of ICCAD, pp 288-294, Nov. 1996. 
[12] I. M. Elfadel and D. D. Ling, "A block rational Arnoldi algorithm for multiport passive model order reduction for multiport RLC networks," in Proc. IEEE/ACM ICCAD, pp.66-71, Nov. 1997.

[13] A. Odabasioglu, M. Celik, L. T. Pileggi, "PRIMA: passive reduced-order interconnect macromodeling algorithm," IEEE Trans. Computer-Aided Design, vol. 17, pp. 645-654, Aug. 1998.

[14] K. Kamon, F. Wang and J. White, "Generating nearly optimal compact models from Krylov subspace based reduced-order models," IEEE Trans. Circuits Systems II, vol. 47, pp. 239-248, April 2000.

[15] J. R. Phillips, L. Daniel, L. M. Silveria, "Guaranteed Passive Balancing Transformations for Model Order Reduction," LEEE Trans. on Computer-Aided Design of Integrated Circuits and Systems, vol. 22, pp. 1027-1041, Aug. 2003.

[16] R. Achar, P. K. Gunupudi, M. S. Nakhla and E. Chiprout, "Passive interconnect reduction algorithm for distributed/measured networks," IEEE Trans. Circuits Systems II, vol. 47, pp. 287-301, April 2000.

[17] P. K. Gunupudi, M. S. Nakhla, "Nonlinear circuit-reduction of high-speed interconnect network networks using congruent transformation," IEEE Trans, on Advanced Packaging, vol. 24, Aug. 2001.

[18] T. R. Arabi, A. T. Murphy, T. K. Sarkar, R. F. Harrington and A. R. Djordjevic, "On the modeling of conductor and substrate losses in multiconductor, multidielectric transmission lines systems," IEEE Trans. Microwave Theory Tech., vol. 39, pp 1090-1097, July 1991.

[19] T. Dhane and D. Zutter, "Selection of lumped element models for coupled lossy transmission lines," IEEE Trans. Computer-Aided Design, vol. 11, pp 957-067, July 1992.

[20] A. R. Djordjevic, B. D. Reljin, D. V. Tosic, T. K. Sarkar, "Transmission-Line Theory Approach to Solution of State Equations for Linear-Lumped Circuits," IEEE Trans. Microwave Theory Tech., vol. 44, pp 479-482, July 1991.

[21] F. H. Branin, Jr., "Transient analysis of lossless transmission lines," Proceedings of the IEEE, vol. 55, pp. 2012-2013, 1967. 
[22] A. J. Gruodis and C.S. Chang, "Coupled lossy transmission line characterization and simulation," IBM Journal of Research and Development, vol. 25, pp 25-41, Jan. 1981.

[23] F. Y. Chang, "The generalized method of characteristics for wave-form relaxation analysis of coupled transmission lines," IEEE Trans. Microwave Theory Tech., vol. 37, pp. 2028-2038, Dec. 1989.

[24] S. Lin and E. S. Kuh, "Transient simulation of lossy interconnects based on the recursive convolution formulation," IEEE Trans. Circuits Systems I, vol. 39, pp. 879-892, Nov. 1992.

[25] D. B. Kuznetsov and J. E. Schutt-Ainé, "Optimal transient simulation of transmission lines," IEEE Trans. Circuits Systems I, vol. 43, pp. 110-121, Feb. 1996.

[26] S. Grivet-Talocia, F. G. Canavero, "TOPLine: a delay-Pole-Residue Method for the Simulation of Lossy and Dispersive Interconnects," Proc. IEEE Electrical Performance of Electronics Packaging, pp. 359-363, Oct. 2002.

[27] W. T. Beyene, J. E. Schutt-Ainé, "Accurate frequency-domain modelling and efficient simulation of high-speed packaging interconnects," IEEE Trans. Microwave Theory Tech., vol. 45 pp. 1941-1947, Oct. 1997.

[28] O. A. Palusinski, A. Lee, "Analysis of transients in nonuniform and uniform multiconductor transmission lines," IEEE Trans. Microwave Theory Tech., vol. 37, pp. 127-138, Jan. 1989.

[29] M. Celik, A. C. Cangellaris and A. Yaghmour, "An all-purpose transmission-line model for interconnect simulation in SPICE," IEEE Trans. Microwave Theory Tech., vol. 45, pp. 1857-1867, Oct. 1997.

[30] M. Raugi, "Wavelet transform solution of MTL transients," IEEE Trans. Magnetics, vol. 35, pp. 1554-1557, May 1999.

[31] S. Barmada ans M. Raugi, "Transient numerical solutions of nonuniform MTL equations with nonlinear loads with wavelet expansion in time or space-domain," IEEE Trans. Circuits Systems I, vol. 47, pp. 1178-1190, Aug. 2000. 
[32] A. Cangellaris, S. Pasha, J. Prince and M. Celik, "A new discrete time-domain model for passive model order reduction and macromodeling of high-speed interconnections," IEEE Trans. Comp. Packag., Manufact. Tech., pp. 356-364, Aug. 1999.

[33] Q. Yu, J. M. L. Wang, E. S. Kuh, "Passive multipoint moment matching model order reduction algorithm on multiport distributed interconnect networks," IEEE Trans. Circuits Systems. I, vol. 46, Jan. 1999.

[34] C. Yen, Z. Fazarinc and R. L. Wheeler, "Time-domain skin-effect model for transient analysis of lossy transmission lines," Proceedings of the IEEE, vol. 70, pp 759-757, July 1982.

[35] T. Vu Dinh, B. Cabon and J. Chilo, "Time domain analysis of skin effect on lossy interconnects," Electronics Letters., vol. 26, pp 2057-2058, Sept. 1990.

[36] C. D. Taylor, R. S. Satterwhite, and C. W. Harrison, "The response of a terminated two-wire transmission line excited by a nonuniform electromagnetic field," IEEE Trans. Antennas Propagation, vol. 13, pp.987-989, Nov. 1965.

[37] T. Wuyts and D. Zutter, "Circuit model for plane-wave incidence on multiconductor transmission lines," IEEE Trans. Electromag. Compat., vol 36, pp. 206-212, Aug. 1994.

[38] C. R. Paul, “A SPICE model for multiconductor transmission lines excited by an incident electromagnetic field," IEEE Trans. Electromag. Compat., vol. 36, No. 4, pp. 342-354, Nov. 1994.

[39] R. K. Das and W. T. Smith, "Incident field coupling analysis of multiconductor transmission lines using asymptotic waveform evaluation," Proc. IEEE Intl. Symp. Electromag. Compat., pp. 265-270, Aug. 1996.

[40] R. Khazaka and M. Nakhla, "Analysis of high-speed interconnects in the presence of electromagnetic interference," IEEE Trans. Microwave Theory Tech., vol. 46, no. 7, pp. 940-947, Jul. 1998.

[41] I. Erdin, R. Khazaka and M. Nakhla, "Simulation of High-Speed Interconnects in a Multilayered Medium in the presence of Incident Field," IEEE Trans. Microwave Theory Tech., vol. 46, no. 7, pp. 2251-2257, Dec. 1998 
[42] I. Erdin, R. Achar and M. Nakhla, "Circuit Analysis of Electromagnetic Radiation and Field Coupling Effects for Networks with Embedded Full-wave Modules," IEEE Trans. Electromag. Compat.,vol 42, pp. 449-460, Nov. 2000.

[43] Q. Zhang, S. Lum, M. Nakhla, "Minimization of delay and crosstalk in high-speed VLSI interconnects," IEEE Trans. Microwave Theory. Tech., vol. 40, pp. 15551563, July 1992.

[44] S. Lum, M. Nakhla and Q. Zhang, "Sensitivity analysis of lossy coupled transmission lines with nonlinear terminations," IEEE Trans. Microwave Theory Tech., vol. 42, pp 607-615, Apr. 1994.

[45] C. Jiao, A. C. Cangellaris, A. M. Yaghmour and J. L. Prince, "Sensitivity analysis of multiconductor transmission lines and Optimization for high-speed interconnect circuit design," IEEE Trans. on Advanced Packaging, vol. 23, pp 132141, May 2000.

[46] A. Dounavis, X. Li, M. Nakhla and R. Achar, "Passive Closed-Form TransmissionLine Model for General-Purpose Circuit Simulators," IEEE Trans. on Microwave Theory and Techniques, vol. 47, pp. 2450-2459, Dec. 1999.

[47] A. Dounavis, X. Li, M. Nakhla and R. Achar, "Passive Closed-Form Transmission Line Model for General Purpose circuit simulators," IEEE MTT-S International Microwave Symposium Digest, vol. 1, pp 377-380. June 1999.

[48] A. Dounavis, Time domain macromodels for high speed interconnects, M. Eng. Thesis, Carleton University, Jan. 2000.

[49] A. Dounavis, R. Achar and M. Nakhla "A General Class of Passive Macromodels for Lossy Multiconductor Transmission Lines", IEEE Trans. on Microwave Theory and Techniques, vol. 49, pp. 1686-1696, Oct 2001.

[50] A. Dounavis, R. Achar and M. Nakhla, "Passive Macromodels for Distributed High Speed Interconnects," European Conference on Circuit theory and Design, vol 3, pp 41-44, Aug. 2001.

[51] A. Dounavis, R. Achar and M. Nakhla, "Passive closed-form time-domain macromodels for on-chip distributed RC interconnects," Proc. IEEE Custom Integrated Circuits Conference, pp. 509-512, May 2002. 
[52] A. Dounavis, R. Achar and M. Nakhla, "Efficient Passive Circuit Models for Distributed Networks with Frequency-Dependent Parameters," IEEE Trans. on Advanced Packaging, vol. 23, pp. 382-392, August 2000.

[53] P. K. Gunupudi, R. Achar, M. Nakhla and A. Dounavis, "Simulation of distributed interconnects with frequency dependent parameters using Krylov Space techniques," European Conference on Circuit Theory and Devices, vol. 1, pp. 425428, Sept. 1999.

[54] A. Dounavis, E. Gad, R. Achar and M. Nakhla, "Passive Model Reduction of Multiport Distributed Interconnects," IEEE Trans. on Microwave Theory and Techniques, vol. 48, pp. 2325-2334, Dec. 2000.

[55] E. Gad, A. Dounavis, M. Nakhla and R. Achar, "Passive Model Order Reduction of Multiport Distributed Interconnects," Proceeding Design Automation Conference, vol. 37, pp 526-531, June 2000.

[56] A. Dounavis, E. Gad, R. Achar and M. Nakhla, "Passive Model-Reduction of Distributed Networks with Frequency-Dependent Parameters," IEEE MTT-S International Microwave Symposium Digest, vol 3, pp 1789-1792, June 2000.

[57] A. Dounavis, P. K. Gunupudi, E. Gad, R. Khazaka, R. Griffith, R. Achar and M. Nakhla, "Addressing Transient Analysis of Multiport Distributed Interconnects," Proc. IEEE Electrical Performance of Electronic Packaging, pp 243-246, Oct. 2000.

[58] P. Gunupudi, R. Khazaka, A. Dounavis, R. Achar and M. Nakhla "Global multilevel reduction technique for nonlinear simulation of high-speed interconnects," Proc. IEEE Electrical Performance of Electronics Packaging, pp 259-262, Oct. 2001

[59] A. Dounavis, R. Achar and M. Nakhla "Efficient Sensitivity Analysis of Lossy Multiconductor Transmission Lines with Nonlinear Terminations", IEEE Trans. on Microwave Theory and Techniques, vol. 49, pp. 2292-2299, Dec 2001.

[60] A. Dounavis, R. Achar and M. Nakhla, "Efficient Sensitivity Analysis of Lossy Multiconductor Transmission Lines with Nonlinear Terminations," IEEE MTT-S International Microwave Symposium Digest, pp 2099-2102, June 2001. 
[61] A. Dounavis, R. Achar and M. Nakhla, "A General Class of Passive Macromodels for Efficient Sensitivity Analysis of High-Speed Distributed Interconnects with Nonlinear Terminations," IEEE 8th International Conference on Electronics, Circuits and Systems, pp 899-902, Sept. 2001.

[62] A. Dounavis, R. Achar and M. Nakhla, "Addressing Transient Errors in Passive Macromodels of Distributed Transmission Line Networks," IEEE Transactions on Microwave Theory and Techniques, vol 50, pp 2759-2768, Dec. 2002.

[63] A. Dounavis, R. Achar and M. Nakhla, "On passive time-domain macromodels of distributed transmission line networks," IEEE MTT-S International Microwave Symposium Digest, vol. 2, pp. 975-978, June 2002.

[64] I. Erdin, A. Dounavis, R. Achar and M. Nakhla, "A SPICE Model for Incident Field Coupling to Lossy Multiconductor Transmission Lines", IEEE Trans. Electromagnetic Compatibility," vol. 43, pp. 485-494, Nov. 2001.

[65] I. Erdin, A. Dounavis, R. Achar, M. Nakhla, "Circuit Simulation of Incident Field Coupling to Multiconductor Transmission Lines with Frequency-Dependent Losses," IEEE EMC International Symposium, pp 1084-1087, Aug. 2001.

[66] I. M. Elfadel, H. M. Huang, A. E. Ruehli, A. Dounavis and M. Nakhla "A Comparative Study of Two Transient Analysis Algorithms for Lossy Transmission Lines with Frequency-Dependent Data," IEEE Transactions on Advanced Packaging, vol. 25, pp. 143-153, May 2002.

[67] I. M. Elfadel, H. M. Huang, A. E. Ruehli, A. Dounavis and M. Nakhla "A Comparative Study of Two Transient Analysis Algorithms for Lossy Transmission Lines with Frequency-Dependent Data," Proc. IEEE Electrical Performance of Electronics Packaging, pp 255-258, Oct. 2001

[68] I. M. Elfadel, A. Dounavis, H-M. Huang, M. Nakhla, A. E. Ruehli and R. Achar, "Accuracy and Performance of Passive Transmission Line Macromodels Based on Optimal Matrix Rational Approximations," Proc. IEEE Electrical Performance of Electronics Packaging, pp. 351-354 Oct. 2002.

[69] A. Dounavis, I. M. Elfadel, R. Achar, M. Nakhla, A. E. Ruehli and H-M. Huang, "Lossy Transmission Line Passive Macromodeling Algorithm - Three Case Studies," Proc. IEEE Electrical Performance of Electronics Packaging, pp. 355358, Oct. 2002. 
[70] A. E. Ruehli, "Equivalent circuit models for three dimensional multiconductor systems," IEEE Trans. Microwave Theory and Techniques, vol. 22, pp.216-224, March 1974.

[71] J. Cullum, A. Ruehli, and T. Zhang, "A method of reduced-order modeling and simulation of large interconnect circuits and its application to PEEC models with retardation," IEEE Trans. Circuit and Systems II, vol. 47, pp. 261-273, April 2000.

[72] C. W. Ho, A. E. Ruehli, and P. A. Brennan, "The modified nodal approach to network analysis," IEEE Trans. Circuits and Systems, vol. 22, pp. 504-509, June 1975.

[73] J. Vlach, K. Singhal, Computer Methods for Circuit Analysis and Design. New York: Van Nostrand Reinhold, 1983.

[74] H. W. Jhonson and M. Grahaml, High-Speed Digital Design, Prentice-Hall, Englewood Cliffs, NJ, 1993.

[75] Y. Saad, Iterative Methods for Sparse Linear Systems, PWD Publishing Company, Boston, 1996.

[76] X. Li, A universal closed-form high-speed interconnect model for general purpose circuit simulators, M. Eng. Thesis, Carleton University, Dec. 1999.

[77] L. Weinberg, Network Analysis and Synthesis. New York, NY: McGraw-Hill Book Company Inc., 1962.

[78] E. S. Kuh and R. Rohrer, Theory of Active Linear Networks. San Francisco: Holden-Day Inc., 1967

[79] E. A. Guillemin, Synthesis of Passive Networks. New York, NY: John Wiley and Son Inc., 1957.

[80] D. F. Tuttle, Electrical Networks; Analysis and Synthesis, New York: McGraw, 1965.

[81] R. W. Newcomb, Linear Multiport Synthesis, New York: McGraw 1966.

[82] A. R. Djordjevié and T. K. Sarkar, "Closed-form formulas for frequency-dependent resistance and inductance per unit length of microstrip and strip transmission lines," IEEE Trans. Microwave Theory Techniques, vol. 42 pp. 241-248, Feb. 1994. 
[83] M. B. Bazdar, A. R. Djordjevic, R. F. Harrington and T. K. Sarkar, "Evaluation of Quasi-Static Matrix Parameters for Multiconductor Transmission Lines Using Galerkin's Method", IEEE Trans. Microwave Theory Techniques, vol. 42 pp. 1223-1228, July 1994.

[84] OPTEMID, Optem Engineering Inc., Version 4.3, Calgary, Alberta.

[85] Artech House Inc., LINPAR version 1.2, Feb. 1989.

[86] J. Poltz, "Optimizing VLSI interconnect model for SPICE simulation," J. Analog Integrated Circuits and Signal Processing, vol. 5, Jan. 1994.

[87] A. Deutch et. al., "Modeling and characterization of long on-chip interconnections for high performance microprocessors," IBM J. Res. Develop. vol 39, pp. 547-567, 1995.

[88] U. S. Pillai, Spectrum Estimation and System Identification, New York: SpringerVerlag, 1993.

[89] I. Erdin, Signal Integrity Analysis of High-Speed Circuits with Embedded Electromagnetic Modules, Ph. D. Thesis, Carleton University, Jan. 2001.

[90] T. Kailath, Linear Systems. Toronto: Prentice-Hall Inc., 1980.

[91] B. Friedland, Control System Design: An Introduction to State-Space Methods. New York: McGraw-Hill Inc., 1986.

[92] W. H. Press, S. A. Teukolsky, W. T. Vetterling, and B. P. Flannery, Numerical Recipies in C, 2nd ed., Cambridge, U. K.: Cambridge University Press, 1992.

[93] HSPICE, Avanti Inc., Version 2001.4, Fremont, CA.

[94] HPADS, Agilent Technologies, Version 190.300, Palo Alto, CA.

[95] A. E. Ruehli, A. C. Cangellaris and H-M Huang, "Three Test Problems for the Comparison of Lossy Transmission Line Algorithms", Proc. IEEE Electrical Performance of Electronics Packaging, pp 347-350, Oct. 2002.

[96] E. Gad, Circuit Simulation Techniques via Generalized Krylov Subspaces Methods, $\mathrm{Ph}$. D. Thesis, Carleton University, May 2003.

[97] D. A. Harville, Matrix Algebra from a Statistican's Perspective, New York: Springer, 1997.

[98] F. B. Hildebrand, Introduction to numerical analysis, Dover Publications, Inc, New York, 1987. 